\title{
A GENETIC ALGORITHM TO DEVISE TEST CASE SEQUENCES BASED ON A' STATE MACHINE DIAGRAM AND DATA FLOW INFORMATION
}

By:

Hongyan Chen

\author{
A thesis submitted to the Faculty of Graduate Studies and Research \\ In partial fulfillment of the requirements for the degree of \\ Master of Applied Science
}
Ottawa-Carleton Institute of Electrical and Computer Engineering
Department of Systems and Computer Engineering
Carleton University
Ottawa, Ontario, Canada

April 2009

Copywright (C) 2009 by Hongyan Chen 
Library and

Archives Canada

Published Heritage

Branch

395 Wellington Street

Ottawa ON K1A 0N4

Canada
Bibliothèque et

Archives Canada

Direction du

Patrimoine de l'édition

395 , rue Wellington

Ottawa ON K1A 0N4

Canada

Your file Votre référence

ISBN: 978-0-494-51982-0

Our file Notre référence

ISBN: 978-0-494-51982-0

NOTICE:

The author has granted a nonexclusive license allowing Library and Archives Canada to reproduce, publish, archive, preserve, conserve, communicate to the public by telecommunication or on the Internet, loan, distribute and sell theses worldwide, for commercial or noncommercial purposes, in microform, paper, electronic and/or any other formats.

The author retains copyright ownership and moral rights in this thesis. Neither the thesis nor substantial extracts from it may be printed or otherwise reproduced without the author's permission.
AVIS:

L'auteur a accordé une licence non exclusive permettant à la Bibliothèque et Archives Canada de reproduire, publier, archiver, sauvegarder, conserver, transmettre au public par télécommunication ou par l'Internet, prêter, distribuer et vendre des thèses partout dans le monde, à des fins commerciales ou autres, sur support microforme, papier, électronique et/ou autres formats.

L'auteur conserve la propriété du droit d'auteur et des droits moraux qui protège cette thèse. $\mathrm{Ni}$ la thèse ni des extraits substantiels de celle-ci ne doivent être imprimés ou autrement reproduits sans son autorisation.
In compliance with the Canadian

Privacy A'ct some supporting forms may have been removed from this thesis.

While these forms may be included in the document page count, their removal does not represent any loss of content from the thesis.
Conformément à la loi canadienne sur la protection de la vie privée, quelques formulaires secondaires ont été enlevés de cette thèse.

Bien que ces formulaires aient inclus dans la pagination, il n'y aura aucun contenu manquant.

\section{Canadä}




\begin{abstract}
Recently, a lots of research attention has been paid to Model-Based Testing, in which test cases are derived from the whole or a part of a model that describes static and dynamical behaviors of a system. State-Based Testing is one of the most important research topics in Model-Based Testing. The objective of this work is to research a new state-based testing approach to help software testers detect defects in implementing software systems as early as possible. To this end, the test cases are derived from a UML state machine diagram, the output of the design phase of a typical lifecycle of software development. In this work, we apply a Genetic Algorithm (GA) to select and prioritize these test cases in sequences to satisfy different user constraints such as time and coverage. In particular, the GA is expected to generate test case sequences that increase their cumulative coverage of data flow information contained in operation contracts as quickly as possible. To evaluate the usefulness of our approach, GA-generated test case sequences were compared with randomly-generated test case sequences in terms of data flow coverage and mutant effectiveness. The experimental results demonstrates that the GA-based approach is useful and effective for creating test case sequences to detect defects based on different user constraints.
\end{abstract}




\section{Acknowledgments}

First, I would like to give my sincere thanks and appreciation to Dr. Labiche for all of his support and guidance throughout my research.

I woud like to thank my colleagues in the SQUALL Lab for the kindness support and encouragement.

My warmest thanks to my family for their support and encouragement they have given me. Without them, I would not have been able to get this so far. 


\section{Table of Contents}

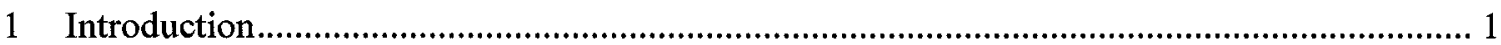

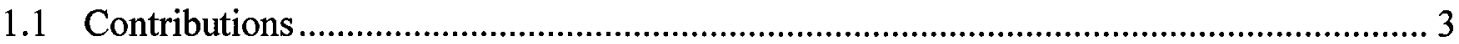

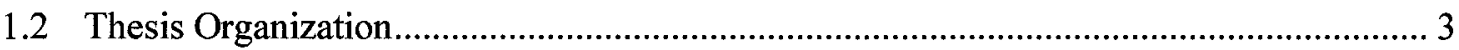

2 Background information on Genetic Algorithms............................................................... 4

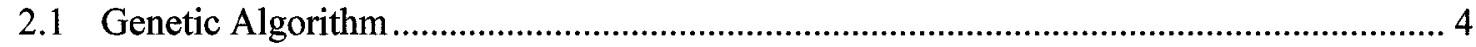

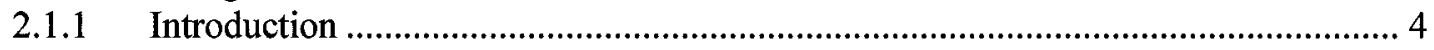

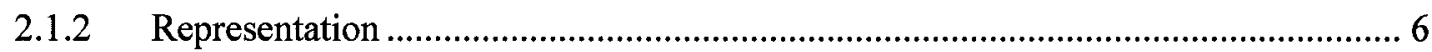

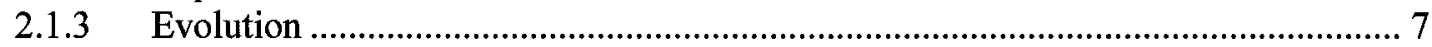

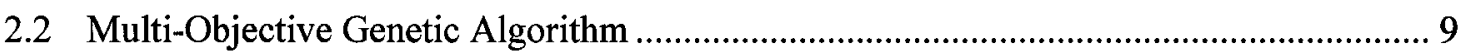

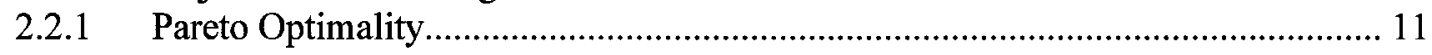

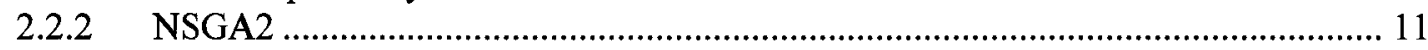

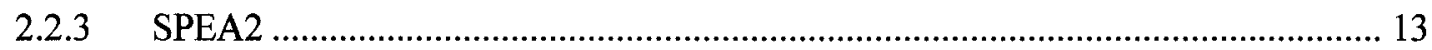

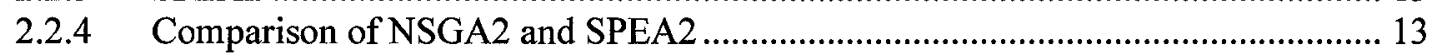

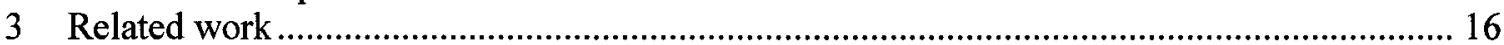

3.1 UML State Machine Diagram and State-based Testing Criteria ................................... 16

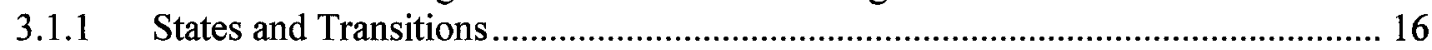

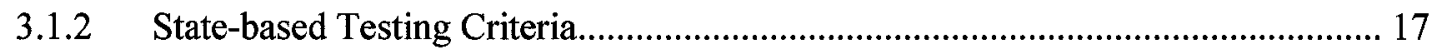

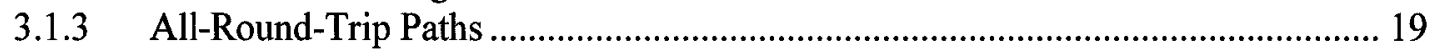

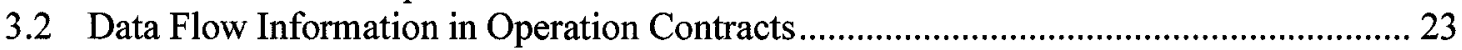

3.3 Related Work on Meta-heuristic Search for Software Testing .......................................25

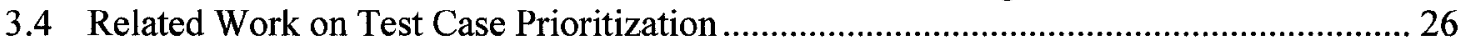

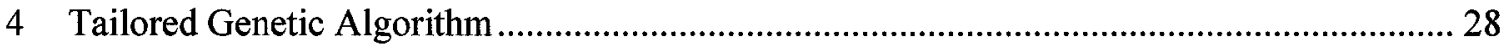

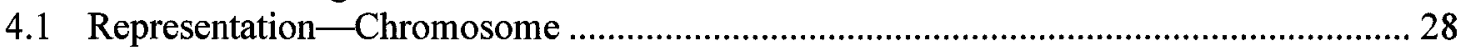

4.1.1 Form a Chromosome by Combining and Splicing All Test Paths......................... 29

4.1.2 Form a Chromosome by Ordering All Test Paths ................................................. 31

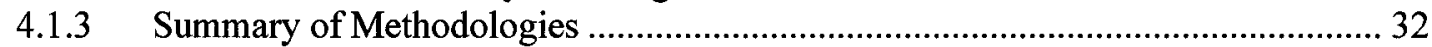

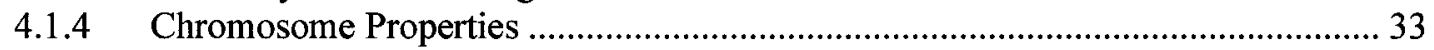

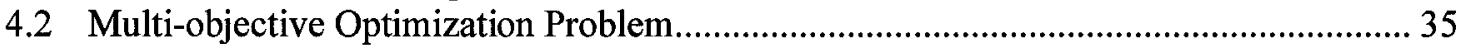

4.2.1 Optimization Scheme 1-Specifying a Maximum Test Cost.................................. 36

4.2.2 Optimization Scheme 2-Specifying a Cumulative Coverage Rate ...................... 37

4.2.3 Optimization Scheme 3-Specifying 100\% Cumulative Coverage Rate and Maximum Available Test Cost.......................................................................... 39

4.2.4 Optimization Scheme 4-Specifying a Maximum Test Cost and a Minimum Cumulative Coverage Rate............................................................................... 40

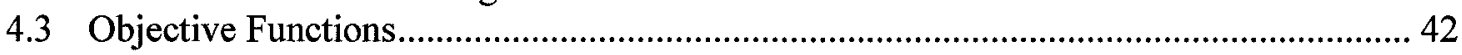

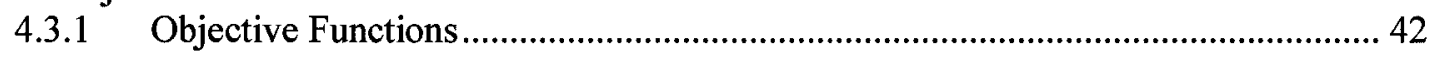

4.3.2 Optimization Scheme 1-Specifying a Maximum Test Cost................................. 45

4.3.3 Optimization Scheme 2-Specifying a Cumulative Coverage Rate ...................... 47

4.3.4 Optimization Scheme 3-Specifying 100\% Cumulative Coverage Rate and Maximum Available Test Cost.......................................................................... 48

4.3.5 Optimization Scheme 4-Specifying a Maximum Test Cost and a Minimum Cumulative Coverage Rate.............................................................................. 49

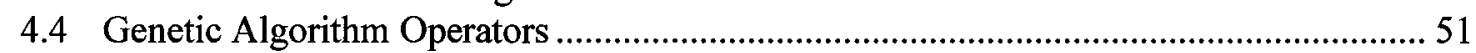

4.4.1 Crossover: Partially matched crossover.............................................................. 51

4.4.2 Mutation (Reordering Mutation): Inversion, Insertion, and Reciprocal ................ 52

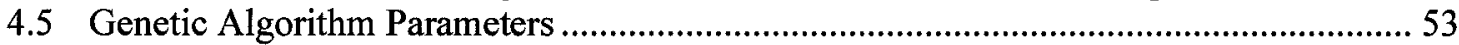

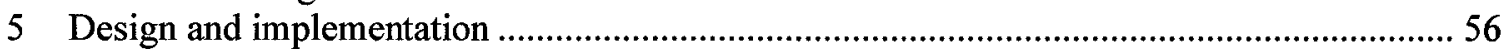




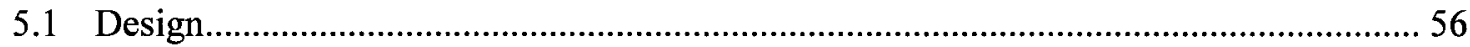

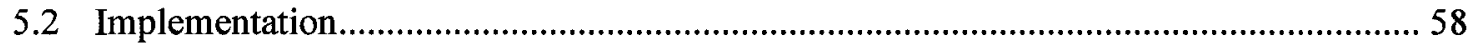

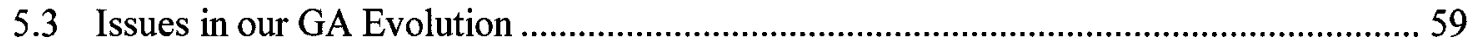

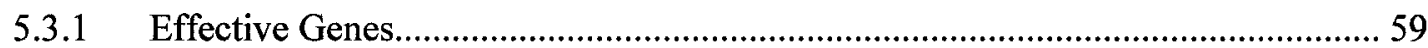

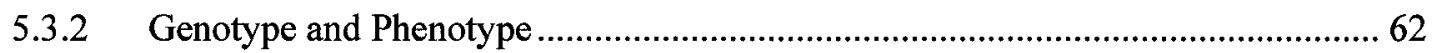

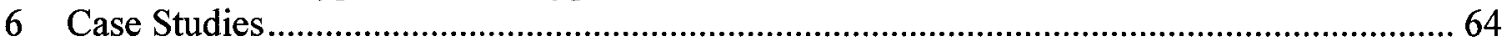

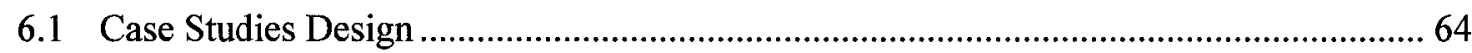

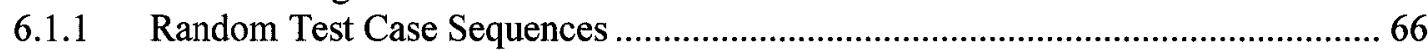

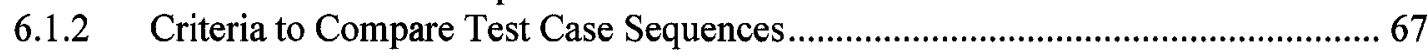

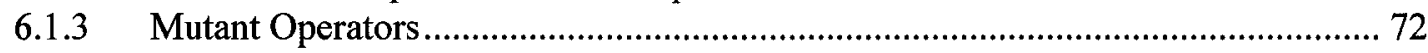

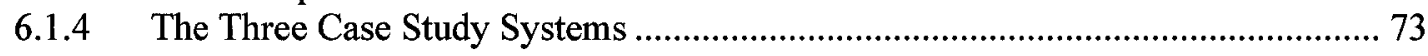

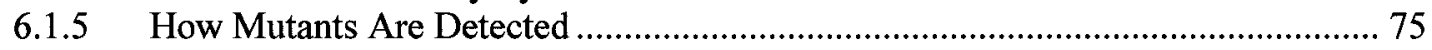

6.2 Cruise Control System Case Study - Results ................................................................... 75

6.2.1 Cruise Control: Optimization Scheme 1-Specifying a Maximum Test Cost....... 76

6.2.2 Cruise Control: Optimization Scheme 2-Specifying a Cumulative Coverage Rate

6.2.3 Cruise Control: Optimization Scheme 3-Specifying 100\% Cumulative Coverage Rate and Maximum Available Test Cost............................................................. 84

6.2.4 Cruise Control: Optimization Scheme 4-Specifying a Maximum Test Cost and a Minimum Cumulative Coverage Rate ................................................................ 86

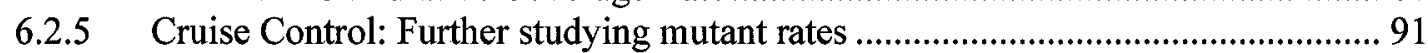

6.2.6 Cruise Control: Summary of Results ................................................................95

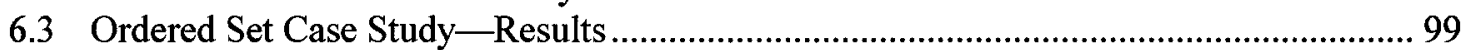

6.3.1 Ordered Set: Optimization Scheme 1-Specifying a Maximum Test Cost ......... 100

6.3.2 Ordered Set: Optimization Scheme 2-Specifying a Cumulative Coverage Rate 109

6.3.3 Ordered Set: Optimization Scheme 3-Specifying 100\% Cumulative Coverage Rate and Maximum Available Test Cost............................................................ 112

6.3.4 Ordered Set: Optimization Scheme 4-Specifying a Maximum Test Cost and a Minimum Cumulative Coverage Rate ............................................................... 114

6.3.5 Ordered Set: Study of the variance between GA runs ....................................... 118

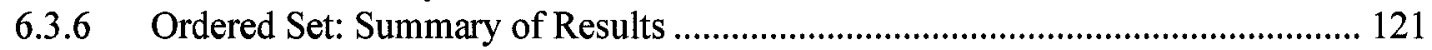

6.4 VCR System Case Study—Results ............................................................................... 122

6.4.1 VCR: Optimization Scheme 1-Specifying a Maximum Test Cost ...................... 122

6.4.2 VCR: Optimization Scheme 2-Specifying a Cumulative Coverage Rate.......... 127

6.4.3 VCR: Optimization Scheme 3-Specifying 100\% Cumulative Coverage Rate and Maximum Available Test Cost......................................................................... 130

6.4.4 VCR: Optimization Scheme 4-Specifying a Maximum Test Cost and a Minimum

Cumulative Coverage Rate .......................................................................... 132

6.4.5 VCR: Study of the variance between GA runs ................................................. 136

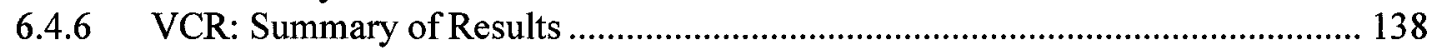

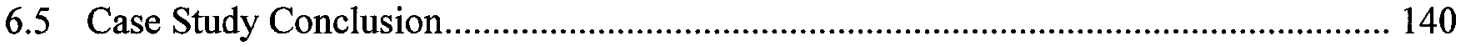

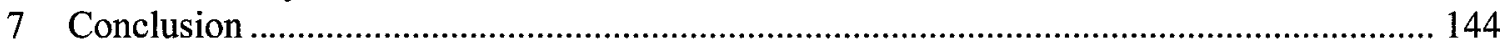

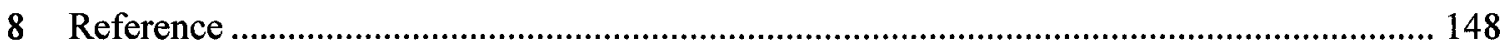

Appendix A Multi-objective Genetic Algorithms ........................................................... 153

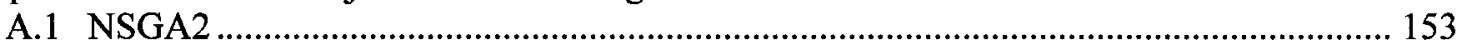

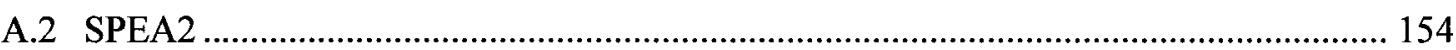

Appendix B The method to Identify All Definitions or All DU Pairs Covered by a

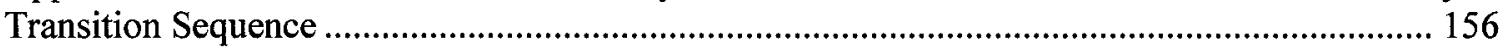

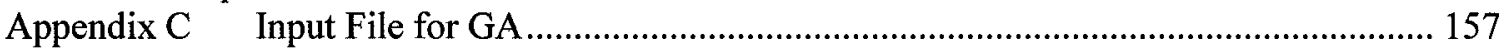

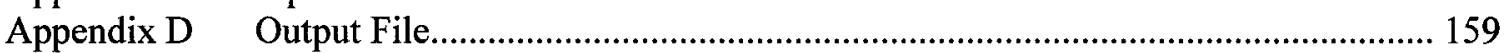

Appendix E The Three Case Studies (System, Test Suite, Mutants) .................................... 160 


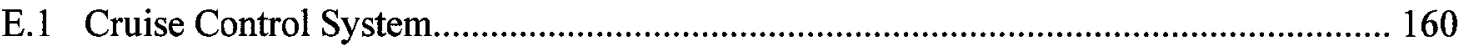

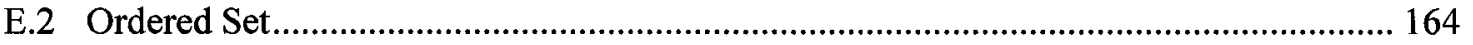

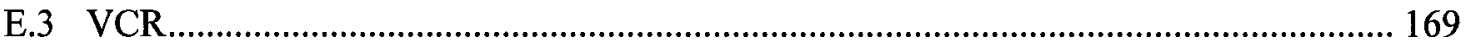

Appendix F Operation Contracts for the Three Case Studies.................................................... 173

F.1 Operation contracts of the Cruise Control System ......................................................... 173

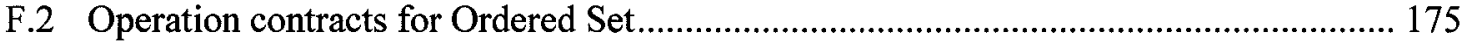

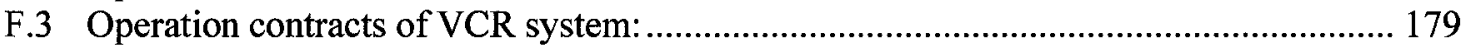




\section{List of Figures}

Figure 1: Genetic algorithm

Figure 2: A simple state machine diagram (A) and the derived transition trees: by depth-first

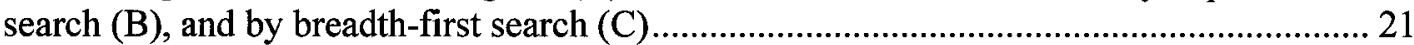

Figure 3: A state machine diagram (A), a transition tree derived following Binder's rule (B) and a

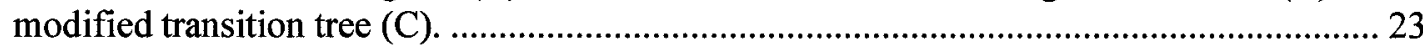

Figure 4: A simple state machine diagram and a test suite satisfying the all-round-trip path

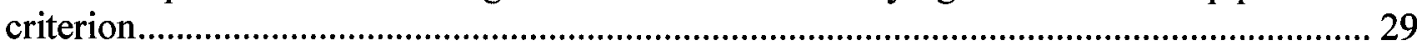

Figure 5: A state machine diagram with a guard condition and a test suite ................................. 30

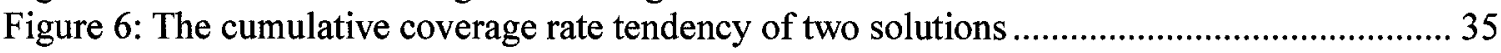

Figure 7: With a given test cost, an example with two possible solutions .................................. 37

Figure 8: An example of two possible solutions reaching the given cumulative coverage rate of

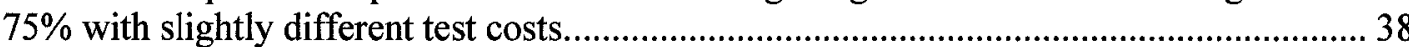

Figure 9: An example of two possible solutions reaching the given cumulative coverage rate of

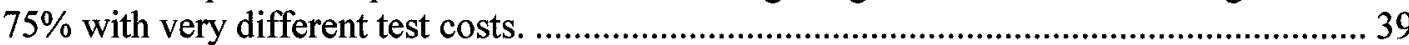

Figure 10: With a 100\% cumulative coverage rate; an example with two possible solutions. .... 40

Figure 11: Four possible solutions in optimization scheme 4 .................................................. 41

Figure 12: Maximize the sum of cumulative coverage rates with a given test cost 16 ............... 43

Figure 13: Minimize the shadowed area with a given cumulative coverage rate $80 \%$................ 44

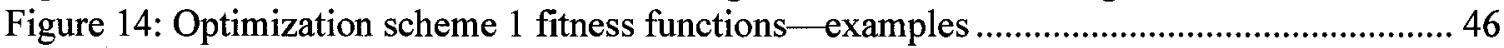

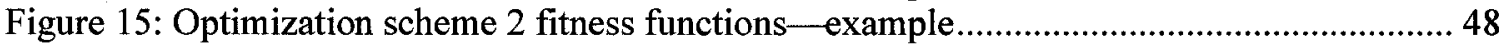

Figure 16: Optimization scheme 3 fitness functions - example..................................................... 49

Figure 17: Optimization scheme 4 fitness functions — example....................................................5 50

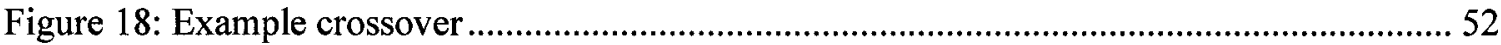

Figure 19: Model of test case sequence and data flow information ............................................56

Figure 20: Algorithm for evaluating coverage of data flow information ....................................57

Figure 21: Class diagram of the implementation based on JGAP framework ............................59

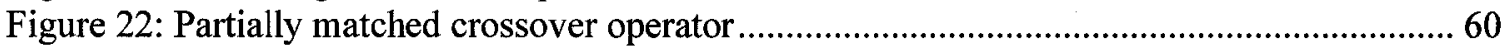

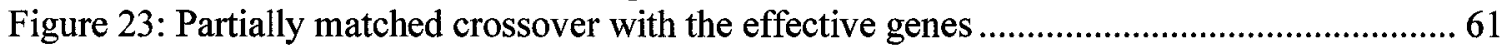

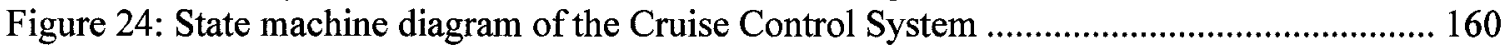

Figure 25: Modified transition tree for Cruise Control System................................................... 161

Figure 26: Analysis of 350 mutants killed by 20 test cases for Cruise Control System............... 162

Figure 27: Analysis of 234 mutants killed by 20 test cases for Cruise Control System.............. 162

Figure 28: Mutant operator distribution for Cruise Control System ....................................... 163

Figure 29: Mutant distribution per method in source code, before (234) and after (157) balancing

Figure 30: State machine diagram of Ordered Set ............................................................. 165

Figure 31: Incomplete transition tree for Ordered set ........................................................ 166

Figure 32: Analysis of 761 mutants killed by 165 test cases for Ordered Set........................... 167

Figure 33: Analysis of 575 mutants killed by 165 test cases for Ordered Set........................... 168

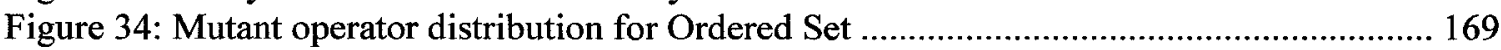

Figure 35: State machine diagram for VCR system ........................................................... 170

Figure 36: Analysis of 450 mutants killed by 1548 test cases for VCR System ........................ 171

Figure 37: Analysis of 175 mutants killed by 1548 test cases for VCR system.......................... 172

Figure 38: Mutant operator distribution for VCR System..................................................... 172 


\section{List of Tables}

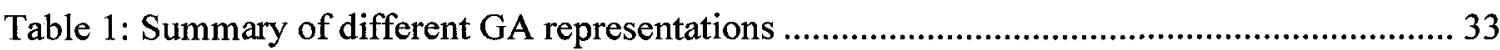

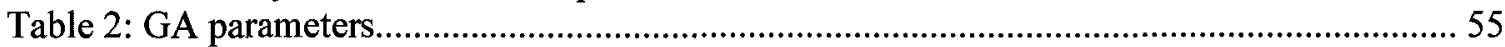

Table 3: A short example: a test suite with 10 test cases covers 20 data flow pieces ................... 60

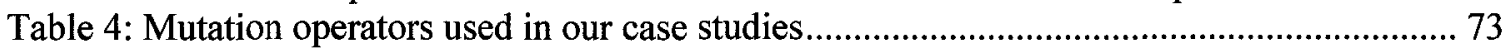

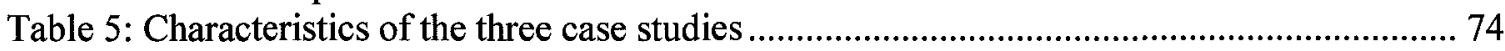

Table 6: Data flow coverage and mutant detection rates of GA-evolved and random sequences

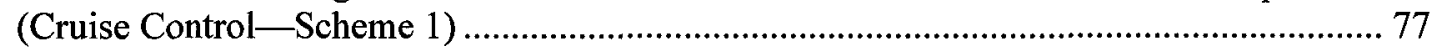

Table 7: Cruise Control Test Set and its data flow/mutant relationship .................................... 81

Table 8: Data flow coverage and mutant detection rates of GA-evolved and random sequences (Cruise Control-Scheme 2)

Table 9: Data flow coverage and mutant detection rates of GA-evolved and random sequences (Cruise Control-Scheme 3) ................................................................................................ 84

Table 10 : Data flow coverage and mutant detection rates of GA-evolved and random sequences

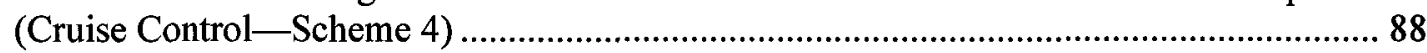

Table 11: Data flow coverage and mutant detection rates of GA-evolved and random sequences with new Ts (Cruise Control-Scheme 4)......

Table 12: Cruise Control Test Set and its data flow/mutant relationship after balancing mutant distribution

Table 13: Data flow coverage and mutant detection rates of GA-evolved and random sequences with different mutant sets (Cruise Control-Scheme 3)

Table 14: the trade-off objective values of the GA-evolved solutions

Table 15: Data flow coverage and mutant detection rates of GA-evolved and random sequences (Ordered Set-Scheme 1)

Table 16: Data flow coverage and mutant detection scores of GA-evolved and random sequences (Ordered Set-Scheme 2).

Table 17: Data flow coverage and mutant detection rates of GA-evolved and random sequences (Ordered Set-Scheme 3).

Table 18: Data flow coverage and mutant detection rates of GA-evolved and random sequences (Ordered Set-Scheme 4).

Table 19: Data flow coverage and mutant detection scores of GA-evolved and random sequences with new Ts (Ordered Set-Scheme 4)

Table 20: Data flow rate and mutant rate in comparison with $20 \mathrm{GA}$-evolved sequences from 20 trials and 20 random sequences-OrderSet System..................................................... 120

Table 21: Data flow coverage rate and mutant score comparison with GA-evolved sequences and random sequences (VCR-Scheme 1)

Table 22: Data flow coverage rate and mutant score comparison with GA-evolved sequences and random sequences (VCR - Scheme 2)

Table 23: Data flow coverage rate and mutant score comparison with GA-evolved sequences and random sequences (VCR-Scheme 3 )

Table 24: Data flow coverage rate and mutant score comparison with GA-evolved sequences and random sequences (VCR-Scheme 4)

Table 25: Data flow coverage rate and mutant score comparison with GA-evolved sequences and random sequences (VCR-Scheme 4), new Ts.

Table 26: Data flow rate and mutant rate in comparison with 20 GA-evolved sequences from 20 trials and 20 random sequences -VCR System 


\section{INTRODUCTION}

Software engineers are under severe pressure due to the short release cycles expected in today's software market. The earlier software testers detect defects, the more time software developers gain to correct them. To find the defects earlier, software testers can schedule the execution of test cases in such a way that test cases with higher defect detection capabilities are scheduled earlier and those with lower defect detection capabilities are scheduled later. By doing this, when time is limited, software testers are able to decide which test cases to execute in order to (hopefully) detect as many defects as possible. If deriving this order could be done automatically, it would greatly reduce the burden of the software testers.

In this thesis, we address the problem of ordering the execution of black-box test cases, and propose a heuristic searching technique for obtaining an approximate optimal test case sequence. The sequence is the most promising for detecting defects as early as possible.

In the context of object oriented development, the Unified Modeling Language (UML) is a standardized specification language for object modeling. UML state machine diagrams are extensions of finite state machines with notations for concurrency, hierarchy and communication. State machine diagrams are commonly used to describe the behavior of a state-dependent System Under Test (SUT) object and other entity, such as a subsystem. They specify the actions a SUT object takes and the sequence of states it goes through in response to internal or external events. State-based testing has received a great deal of research attention. A number of papers propose using state machine diagrams as test models to derive test suites (e.g., [1, 2]). One of those techniques, referred to as Binder's round-trip path tree, has shown to be an interesting cost-effective compromise between inexpensive but ineffective criteria (such as the all-transitions criterion) and very effective but very expensive criteria (such as the all-transition pairs and the full predicate criteria) [3]. 
Furthermore, for UML-based analysis and design, operation contracts are widely used to specify the conditions that need to be satisfied before an operation is invoked (precondition) and the changes that will happen after an operation executes (post-condition). Contracts are agreements between callers and callees to specify their rights and obligations [4]. From a testing point of view, although a tester may not know exactly how an operation is implemented, he/she may still be able to ascertain the main data changes and uses of class attributes and operation parameters from operation contracts. To that end, some rules have been defined by Briand et al. [5] to identify the data flow information entailed in the operations from their contracts. Furthermore, Briand et al's work reveals that the data flow information could be a good indicator of defect detection effectiveness.

From the above two aspects, we believe that with a test suite derived from a state machine diagram and the data flow information contained in the operation contracts, software testers can ascertain the possible data flow information in the implementation. Once they have the possible data flow information, software testers can speculate on the probability of detecting defects for each test case. Furthermore, software testers can schedule test cases so that the test case with the highest probability of detecting defects is executed first and the test case with the lowest probability of detecting defects is executed last.

In this thesis, given a state-based, black-box test suite, built according to the round-trip path strategy for example, we propose a method for ordering the test cases of the test suite. The ordering process relies primarily on the data flow coverage of those test cases, for the reasons discussed above. The ordering of test cases is subject to constraints. Given the current software development context, which is subject to tight deadlines and resources (e.g., time cost), we intend to find sequences of test cases that achieve a maximum data flow coverage rate and/or pay a minimum time cost (e.g., in terms of calls to the SUT to be performed in the sequence). The problem then becomes multi-objective: to maximize data flow coverage rate and minimize execution cost. We propose solving it using a multi-objective genetic algorithm. 
The proposed approach is evaluated on three representative systems that exhibit statebased behaviors: two purely event-based real-time systems (a cruise control system and a VCR system) and a data structure class (an ordered set class).

\subsection{Contributions}

This thesis makes three major contributions.

First, this thesis proposes an approach for ordering test cases from a test suite derived from specifications entailing a representation of state based behavior and operation contracts. This approach allows software testers to plan testing earlier in the development process.

Second, it suggests a heuristic method for obtaining a subset of test cases in an order that satisfies a software tester's specification, with a compromise on execution cost and defect detection effectiveness.

Third, it empirically validates the approach on representative case study systems.

\subsection{Thesis Organization}

The rest of the thesis is organized as follows: Chapter 2 presents the background information for this work; Chapter 3 introduces some related works; Chapter 4 describes how a genetic algorithm is applied to our problem; and Chapter 5 presents the design and implementation of our approach. Chapter 6 provides three case study results. Chapter 6 summarizes this research. 


\section{BACKGROUND INFORMATION ON GENETIC ALGORITHMS}

This chapter presents background information to help readers understand this thesis better. Section 2.1 presents a general description of genetic algorithms. Section 2.2 discusses the most popular techniques for multi-objective genetic algorithms.

\subsection{Genetic Algorithm}

A genetic algorithm is a searching technique for finding approximate optimal solutions based on the mechanics of natural selection and natural genetics [6]. A genetic algorithm is a random search directed by its fitness function toward regions with likely improvement. (A fitness function indicates the level of adaptation that an individual is suitable to a specific environment [7].) In the initial phase, solutions are spread randomly throughout the search space. During the evolution, the searching process will explore more search space and identify promising optimal areas. Gradually, the solutions will converge towards a relative optimal area. A genetic algorithm is a heuristic algorithm that will not necessarily lead to optimal solutions. Section 2.1 .1 provides a general introduction on Genetic Algorithms. Sections 2.1.2 and 2.1.3 discuss the notions of solution representation and population evolution, respectively.

\subsubsection{Introduction}

A Genetic Algorithm (GA) is inspired by biological evolution. It is used in optimization and search problems. It takes advantage of the characteristics of biological evolution, such as inheritance, recombination, mutation and selection, to adapt individuals in a population to a specific environment. The main idea is that a fitter individual in an environment will have a greater opportunity to survive in competition and pass its own heredity material on to the next generation. A simple genetic algorithm, summarized on the basis of the introduction in [6], is shown in Figure 1. 
Genetic Algorithm:

Step 1: Randomly initialize a solution set with size N.

Step 2: Evaluate fitness value for each solution.

Step 3: Select solutions to produce offspring.

Selection methods: roulette wheel, tournament, rank, elitism, etc.

Step 4: Produce offspring by crossover and mutation.

Crossover methods: one point, two points, uniform, position based, etc.

Mutation methods: swap, insert, delete, reverse, etc.

Step 5: Generate next generation and go back to step 2, loop until termination.

Figure 1: Genetic algorithm

In the first step, the genetic algorithm initializes a population of $N$ chromosomes (or individuals). In the second step, the algorithm evaluates the fitness value of chromosomes. In the third and fourth steps, the algorithm selects two (or more) parents based on their fitness (e.g., the best fitness values), mates them (crossover and mutation) to generate two children, puts the children into the child population, then repeats these steps until all slots in the child population are filled. In the fifth step, the algorithm repeats steps 2 to 5 until some stopping criterion is met.

Throughout the process, there are many stochastic decisions, such as a randomly generated initial solution set; parents randomly picked for crossover; a randomly picked crossover point; a randomly picked mutation point, and so on. These stochastic choices are only used as tools to guide a search and are well controlled by the algorithm parameters [6]. Fitness function(s) directs the whole search process. Competition based on fitness values among solutions leads to a superior solution with a greater probability of producing good offspring and an inferior solution with a lower probability of producing good offspring. Directed by fitness function(s), the population becomes better and better. The GA search process starts its intelligent exploitation process from the initial population within a defined search space. The initial solutions randomly spread in the search space. Genetic operators "crossover" and "mutation" produce new solutions, therefore extending the explored area. Fitness function(s) lead solutions towards optimal areas. 


\subsubsection{Representation}

In genetic algorithms, a candidate solution is coded as a chromosome, composed of a set of genes. A gene has two important attributes: its position in the chromosome (or locus) and its values (or allele). Reference to the terms "genes" or "alleles" indicates the genotype of a chromosome (i.e., the chromosome structure [6]). Reference to "objectives" or "fitness values" indicates the phenotype of a chromosome (i.e., the way the individual interacts with its environment [6]).

Let us consider the traveling salesman problem as an example. A salesman is going to travel to a list of cities exactly once, starting and ending in his home city. As a candidate solution, the cities to which he/she is going to travel can be randomly ordered. A sequence of cities is encoded to a chromosome. A gene corresponds to a city. A gene at locus $\mathrm{k}$ means the city in the $\mathrm{k}$-th gene will be visited after $\mathrm{k}-1$ cities have been visited. In this problem, the traveling salesman has to start his trip from home and end at home. As a result, the alleles for the first gene and last gene have only one possible value, the home city. The alleles for all intermediate genes have all cities except the home city as possible values.

Chromosomes in a GA can have a fixed-length, meaning all solutions in the genotype have similar structures, with the same number of genes. For example, in the traveling salesman problem, he/she is going to travel to a fixed number of cities and to each city exactly once. It is obvious that all solutions (chromosomes) contain all cities and contain each one exactly once, so all chromosomes have the same length. Alternatively, chromosomes in a GA can have variable lengths, meaning that some genes are optional. The individuals in a population may have different structures with a different number of genes, thus different chromosome lengths. Let us consider the rural postman problem as an example: when a postman delivers letters along some streets as a subset E' of streets set $E$ (the streets in E' may be disconnected), he/she wants to find the shortest path that covers every street in E' (he/she may re-traverse any street). The possible solutions may contain some streets that are in the street set $\mathrm{E}$ but not in the subset E', or contain a street in $E^{\prime}$ several times. In this problem, the number of streets the postman is going to traverse 
is not a fixed number. In the genetic algorithm, solutions for such a problem could be coded as variable-length chromosomes. Deciding on whether one wants to use fixedlength or variable-length chromosomes has important consequences on genetic operators (Section 2.1.3).

\subsubsection{Evolution}

During evolution, there are three important genetic operators: selection, crossover and mutation. We provide a general discussion of these genetic operators in the following sections. The reader interested in more details is referred to [6].

\subsubsection{Selection}

Selection is a process in which an individual is selected to breed a new generation. Solutions are selected through a fitness-based process, where a fitter solution will be more likely to be selected to breed. Many selection methods are used to select parents; they are different with regard to the way individuals are compared and selected. Some of the most well known and used selection methods are discussed below.

Roulette wheel selection is a stochastic sampling scheme with replacement. The reproduction selection is based on the individuals' fitness proportion in a population. According to De Jong's study [6], for a maximizing problem, "the expected number of offspring for each chromosome is $n^{*} f / \bar{f}$ (assuming that the entire population with $n$ solutions is reproduced at each generation)", where an individual's fitness value is $f$ and the average population fitness value is $\bar{f}$. Through this formula, a fitter individual with a higher fitness value will gain more opportunities to produce offspring. But the roulette wheel selection may cause the population to lose its diversity quickly if the fitness of the best individual is extremely better than the average fitness (in that the majority of the offspring population will be descended from the best individual). In this situation, the roulette wheel selection may cause the population converging towards a near-optimal area. Such a convergence is called a premature convergence [6]. 
With linear rank selection, the population is ranked according to individuals' fitness value for the objective(s), which is called raw fitness. An individual is then selected for breeding based on its rank instead of its raw fitness. In other words, the fitness of an individual in the competition for breeding is its rank. In an environment with a high variance of raw fitness values, the selection pressure can be reduced, which is the ratio of: the best individual's selection probability to the average selection probability of all individuals. With linear rank selection, individuals with extremely good fitness will not dominate a population very quickly. In rank selection, the raw fitness value of individuals is not directly used for breeding, so the difference in raw fitness between individuals does not affect the selection as evidently as roulette wheel selection. However, this method is less efficient since its computation complexity is $O(n \log n)$ (it sorts the whole population).

With tournament selection, in each tournament selection trial, a fixed number of individuals, known as tournament size, are selected randomly from a population. These individuals are then compared according to their fitness value. Within one tournament, the fittest individual wins the tournament with a selection probability. One of the remaining individuals wins the tournament with the left chance. The tournament size and the selection probability are combined to control the selection pressure. In total, for a population of $n$ individuals, $n$ independent trials are needed to select individuals for breeding. This method has some benefits: 1 . knowledge of the individuals within one only tournament is required instead of the whole population; 2 . there is no sorting; 3 . it is easy to control selection pressure by controlling tournament size; 4. it is good for parallel computing.

\subsubsection{Crossover}

Crossover, also referred to as recombination takes two individuals as two parents, exchanges part of the genes from the two parents and forms two offspring. For different chromosome structures, the crossover methods are different.

For fixed-length chromosomes, the following crossover operators can be used. With onepoint crossover, a crossover point for both parent chromosomes is picked randomly and 
all genes beyond that point are swapped between the two parent chromosomes. With twopoint crossover, two crossover points on parent chromosomes are picked randomly and all genes between the two points are swapped between the two parent chromosomes.

These crossover methods can be used for non-ordered chromosomes, i.e., chromosomes in which the order of genes does not matter during the evaluation of fitness functions. For ordered chromosomes, where the fact that a gene appears before another is important during fitness evaluation, partially mapped crossover (PMX) can be applied, which will be discussed in section 4.4.1.

For variable length chromosomes, the "Cut and Splice" crossover method can be applied. With Cut and Splice, each parent chromosome has its own choice of crossover point. The parent chromosome is then cut into two pieces. Each child chromosome takes one piece. A child chromosome is formed by splicing two pieces from parent 1 and parent 2 . The crossover point for each parent chromosome is randomly picked, or pseudorandomly picked if a randomly picked cut and splice point does not result in a feasible offspring.

\subsubsection{Mutation}

Mutation is a genetic operator for maintaining population diversity. It protects some potentially useful genetic materials against irrecoverable loss. In the initial population, a set of chromosomes is randomly generated with genes that contain a specific set of alleles but may not contain all possible alleles. Without mutation, for a gene, the crossover operator only swaps alleles that exist in the current population, but the alleles that do not appear in the initial population will never appear in any generation without mutation operators. During evolution, the mutation operator changes the value of a gene from the current allele to another allele.

\subsection{Multi-Objective Genetic Algorithm}

Real-world optimization problems often involve different criteria. These criteria may be presented simultaneously, be incommensurable, or even conflict with each other. In a 
single objective genetic algorithm, a fitness value is often a simple value to indicate how well a solution is evaluated for a well-defined objective. The genetic algorithm (GA) simply seeks the best (highest or lowest) value. But in a multi-objective genetic algorithm, objective functions are different for different objective criteria. A vector with values for different objectives is used to determine a solution's fitness. Because the fitness value comes from a vector, there are various methods for dealing with the elements of the vector.

The simplest method, a Weighted Sum of Objectives, is to obtain fitness by multiplying each element of the vector with a weight factor and adding all the weighted elements together. This is not satisfactory since the choice of weights can only be performed a priori and can only be subjective.

VEGA [8] is another simple method. VEGA goes through the whole population and selects the fittest solutions for each objective to form a mating population. Inside the mating population, all solutions have the equivalent opportunity to breed offspring. This technique ensures that heuristic optimal solutions for each objective are represented in the population, but it can lead to the population converging towards each objective's optimal area. For instance, with a given multi-objective problem dealing with maximizing profit and minimizing cost in a manufactory, reducing manufacturing cost will reduce the output but reduce profit at the same time. Through genetic evolution by VEGA, the population contains a half of all individuals with maximum profit and a half of all individuals with minimum cost. Ultimately, no compromising solutions could be used to reveal the constraint relationship of maximizing profit and minimizing cost.

To solve this problem, other genetic algorithms evolve solutions using the ParetoOptimal technique (section 2.2.1). A number of multi-objective genetic algorithms are based on the Pareto-Optimal technique, such as SPEA2 [9] (section 2.2.2) and NSGA2 [10] (section 2.2.3) which have been widely used in different domains such as circuit design, business process design, etc. We therefore compare SPEA2 and NSGA2 (section 2.2.4) 


\subsubsection{Pareto Optimality}

Pareto Optimality, formulated by Italian economist Vilfredo Pareto, is a measure of efficiency in multi-criteria problems. In a genetic algorithm, Goldberg [6] mathematically defined a Pareto Optimality, for example for a maximizing problem. Vector point $\bar{x}$ dominates vector point $\bar{y}$, written as $(\bar{x} p>\bar{y})$ where $p>$ means Pareto dominate, if and only if $\left(\forall_{i}\right)\left(x_{i} \geq y_{i}\right) \wedge\left(\exists_{i}\right)\left(x_{i}>y_{i}\right)$. This can be interpreted as follows: if a Pareto-Optimal solution has a fitness vector $f(x)=\left\{f_{1}(x), f_{2}(x), \ldots f_{n}(x)\right\}$, another non-Pareto-Optimal solution has a fitness vector $f(y)=\left\{f_{1}(y), f_{2}(y), \ldots f_{n}(y)\right\}$ where each element of $f(x)$ is greater than or equal to the corresponding element of $f(y)$ and at least one element of $f(x)$ is strictly greater than the corresponding element of $f(y)$. In the objective search space, $f(x)$ dominates $f(y)$; in the decision variable space, solution $x$ dominates solution $y$.

A Pareto frontier is the set of all Pareto-Optimal solutions, which form a trade-off frontier. All solutions on the Pareto frontier cannot evolve for one objective without sacrificing other objective(s).

\subsubsection{NSGA2}

NSGA2 [10] is an extension of Non-dominated Sorting Genetic Algorithm (NSGA) [11]. In NSGA [11], the initial population is generated randomly. NSGA sorts the whole population, finds the first non-dominated frontier (if any one of the objectives of a solution is not dominated by anyone else for the same objective in the current population, this solution is located on the current non-dominated frontier), and assigns a dummy fitness value to all individuals in the current non-dominated frontier. (The dummy fitness value is only a temporary value to identify the frontier that a solution belongs to. It will not be directly used in the selection process). For the current frontier, a niche count is used to evaluate the number of individuals coexisting in a niche. (A niche is a specific sub-domain of environment or area that some individuals live together and share resources [6]. The niche boundary is determined by a user-specified niche diameter, which is also called sharing parameter in some GAs.) For each solution at the current frontier, NSGA calculates its shared fitness value by dividing the assigned dummy fitness 
by its niche count. (Shared fitness is used to balance the population distribution. For example, a good individual living in a crowded area may get less opportunity to survive than a relatively bad individual living in a sparse area.) NSGA then temporarily removes these individuals in the current frontier in order to sort the remaining individuals. NSGA repeats the following steps until all individuals are classified: finds individuals in the next non-dominated frontier from the remaining individuals; assigns a smaller dummy fitness value; calculates these individuals' shared fitness; temporarily removes them. Finally, after all individuals are classified and assigned shared fitness values, NSGA selects and recombines the individuals to produce the next generation according to the shared fitness (see [11] for details).

This algorithm ensures a relative even distribution of individuals and allows for the existence of multiple equivalent solutions. Some researchers [12] show NSGA performed quite well in terms of "coverage" of the Pareto front (i.e., how widely solutions are distributed on the Pareto front). However, this algorithm lacks elitism (an effort to preserve the best solutions), it requires that the sharing be manually set a priori (this value is specified based on user's awareness to the search space), it does not consciously protect the boundary solutions near the Pareto front boundary from being discarded (for example, a solution is extremely good at one objective and extremely bad at another objective), and it has a high computation complexity because it sorts the whole population.

NSGA2 is an improvement of NSGA. It introduces elitism to preserve the best solutions, eliminates the manual specification of the sharing parameter to reduce the algorithm sensitivity to a manually set parameter, and reduces computation complexity to save execution time. It also introduces a comparison mechanism that ensures an even distribution of solutions on the Pareto front. The overall process is similar to the one of NSGA, with some variations (to implement the improvements) that we detail in Appendix A.1. 


\subsubsection{SPEA2}

SPEA2 [9] is an improvement on SPEA [12]. SPEA uses the concept of Pareto dominance to assign a scalar fitness value to each individual. SPEA maintains two populations: a regular population with population size $N$ and an archive population with population size $M$ to save a set of non-dominated solutions (other solutions are saved in the regular population). The fitness value of an individual is determined by comparing the two populations. The fitness value of an individual $i$ in the archive population, which is called strength, equals $n / N+1$ ( $n$ is the number of individuals in the regular population that are dominated by the individual $i ; N$ is the regular population size). The fitness value of an individual $j$ in the regular population equals the value of the summation of strengths of all non-dominated solutions in the archive population that dominate $j$. During the evolution, SPEA maintains the size $M$ of the archive population through a truncation technique if the number of the non-dominated individuals exceeds $M$. (The truncation technique, named "clustering" in [12], divides non-dominated solutions into $M$ groups of relatively homogeneous solutions based on their distance in phenotype and selects one representative solution for each group.) In a genetic evolution, SPEA selects individuals from the regular population and the archive population with a tournament selection to breed offspring.

SPEA2 [13] improves on SPEA in three aspects. First, it improves the fitness assignment scheme for an individual $i$. In SPEA2, the fitness value takes into account the number of individuals that dominate $i$ and the number of individuals $i$ dominates, in both the archive population and the regular population, whereas in SPEA, the fitness assignment of $i$ is only determined by the strengths of its dominators in the archive population. Second, it introduces a nearest-neighbour density estimation technique during truncation. Third, it has a new archive truncation method for preserving boundary solutions. We provide the detail information of this algorithm in Appendix A.2.

\subsubsection{Comparison of NSGA2 and SPEA2}

Both NSGA2 and SPEA2 are elitism algorithms, assign fitness based on Pareto domination, use external sets for selection and use a concept similar to a cluster (or 
niche). A great deal of research (e.g., [14-16]) in different fields, such as performance analysis [17], business processes design and circuit design, has shown that NSGA2 and SPEA2 have similar behaviors with regard to optimization, as well as some slight differences. Based on these research results and the interests of concern to us, we define the following metrics for comparing NSGA2 and SPEA2: computing complexity, solution distribution and diversity, distance from the obtained set to the Pareto-Optimal frontier, performance for different objective dimensions.

Computing complexity is an important benchmark for comparing the performance of algorithms. SPEA2 and NSGA2 have the same computing complexity of $O\left(M N^{2}\right)$ [15], where $M$ is the number of objectives and $N$ is the population size.

Solution distribution/diversity relates to the constraint relationship(s) between objectives. A bad example is VEGA, in which solutions are divided into groups by objectives and crowded to the optimal area for each objective. VEGA does not present compromising solutions so a user cannot have a clear understanding of the constraints between objectives. An algorithm that would present more compromising solutions that are evenly distributed would allow a user to better more understand those constraints between objectives. Additionally, some authors argue that SPEA2 produces broader distributions of solutions than NSGA2 [9], [17], [18].

A genetic algorithm is a heuristic search and therefore does not guarantee that solutions are optimal. The distance to the optimal solution (when it is known) can therefore be used to compare the efficiency of different algorithms. An efficient algorithm is expected to lead solutions close to the known optimal. The study reported in [9] shows that there is no obvious difference between SPEA2 and NSGA2 with this respect.

Similarly, the performance of SPEA2 and NSGA2 for different objective dimensions are very close [9], [19]. However, the comparison by Zitzler [9] indicates that in higher dimensional objective spaces (e.g., four fitness functions and higher), SPEA2 seems to have an advantage over NSGA2. 
In conclusion, the two algorithms are very close to one another: we cannot say either is definitely better than another. We however selected SPEA2 because it retains more diversity, comparatively speaking, and we have an available SPEA2 Java implementation. 


\section{RELATED WORK}

In this chapter, we discuss related work in state-based testing (Section 3.1), data flow identification from operation contracts (Section 3.2), the meta-heuristic searching techniques applied in software testing (Section 3.3), and test case prioritization (Section 3.4).

\subsection{UML State Machine Diagram and State-based Testing Criteria}

The UML state machine diagram notation is widely used to describe the dynamic behaviors of a state-dependent object or subsystem. It shows a state machine, consisting of states, transitions, events and activities, to emphasize the event-ordered behavior of an object or subsystem [20]. A state machine diagram presents the sequence of states an object or a subsystem goes through within its lifespan in response to internal/external events [21].

\subsubsection{States and Transitions}

A state can be characterized in three complementary ways [20]: a set of attribute values; a period of time for which an object waits for an event; and a period of time for which an object carries out ongoing activity. As our research interest is data flow, we focus mainly on the set of attribute values. A state is a unique combination of attribute values (ranges) of an object, known as state invariants. The states in an object's lifespan are subsets of all possible combinations of attribute values. When the attribute values are changed, the state of the object may be redefined according to the new combination of these values. A state can have an entry (resp. exit) action that executes each time the object enters (resp. leaves) the state.

A transition models a movement from one state to another. It is a unique combination of five aspects: a source state, an event trigger, optional guard conditions, effects and a target state [20]. A source state is the movement's start point and a target state is the movement's end point. An event triggers the object state change, moving from the source state to the target state. In other words, an event trigger is a stimulus of a transition. A 
guard condition determines if the stimulus will be accepted and the state will be changed. Effects are some executable behavior, such as a set of actions that will happen in a deterministic order.

\subsubsection{State-based Testing Criteria}

According to [21], state-based testing consists in devising transition sequences (or sequences of stimuli) to exercise during testing, executing them and, at the end of the transition sequences (or after each transition exercised), comparing the resulting states with the expected states. The rationale for focusing on the resulting state (compared to the expected state) is that each state is a unique combination of some attribute values (ranges) in a state machine diagram. By determining the current state instead of determining all attribute values (ranges), a software tester can identify whether the system is responding correctly to a stimulus or a set of stimuli. But there are some difficulties with state-based testing: 1) before a given transition sequence is executed, an object of a SUT class must be placed in the desired start state of the given transition sequence; 2) because of the existence of guard conditions in a state machine diagram, even if a SUT object is placed in the desired start state of a given transition sequence, the stimulus of each transition will not be accepted if guard conditions are not satisfied. As a result, the transition sequence will not be exercised. In practice, to carry out a transition sequence, a software tester has to manually derive a complementary transition sequence in order to set the SUT object at the start state and adapt the attribute values to satisfy guard conditions.

A number of state-based strategies and coverage criteria have been proposed in the literature: all transitions [1, 22], all transition pairs [1], all transition k-tuples [22], full predicates [1, 22], round-trip path tree [22], and complete sequence [1]. (The last criterion is based on software testers' experience, and domain knowledge.)

All-transition coverage is obtained if any transition in a state machine diagram is exercised at least once. As a result, all states, all events and all actions will be exercised at least once. This criterion does not require any specified transition sequence. According 
to Offutt [1], obtaining all-transition coverage uses minimum cost, but the effectiveness of this criterion is no better than random testing.

All transition-pairs coverage is obtained if, for each pair of adjacent transitions in a state machine diagram, a test suite contains a test case to exercise this pair in sequence. In Binder's book [22], a more general coverage criterion is the all transition k-tuples. All ktuples coverage is obtained when every specified transition sequence of $\mathrm{k}$ events is exercised at least once. For example, a 2-tuple coverage includes all transition-pairs; a 3tuple coverage includes all triples; and so on. Transition-pairs coverage is intended to check the interfaces among states [1] and some incorrect or corrupt states can be detected by this coverage criterion [22]. Compared with all-transition coverage, this criterion has a much greater execution cost. See [3] for detailed discussion on this issue.

According to Offutt [1], to obtain full predicates coverage, for each predicate $P$ on each transition, a test suite must include test cases that cause each clause $c$ in $P$ to result in a pair of outcomes, true and false, where the value of $c$ controls the value of $P$. This coverage criterion exerts a greater execution cost and is more effective than all-transition. Offutt et al. [2] also present general criteria for generating test inputs from state-based requirements/specifications. In their example, they show their full-predicate technique achieves a high level of branch coverage and detects a large percentage of faults.

A well-known criterion is Binder's round-trip path tree [22]. In 1978, Chow proposed a set of coverage criteria for finite state machines (FSMs), one of them being the n-switch cover. In 1999, Binder adapted this coverage criterion to state machine diagrams and came up with the all-round-trip paths coverage criterion, in which every sequence of specified transitions beginning and ending in the same state is exercised at least once. The set of all-round-trip paths includes all transition sequences with $\mathrm{k}$ transitions where $\mathrm{k}$ is less than or equal to the number of states. Binder presented an algorithm to build a round-trip path tree with an adequate test suite covering all-round-trip paths. It is referred to as the transition tree or Binder's transition tree in the sections below. According to Briand et al. [3], this test criterion is a good compromise in terms of defect detection 
effectiveness and execution cost between all transitions and all transition pairs (or full predicate).

\subsubsection{All-Round-Trip Paths}

Round-trip paths are defined by Binder [22] as "transition sequences that begin and end with the same state and simple paths from the initial state to final state. A path is a sequence of transitions: state $_{p}$, event $_{i}$, state $_{q}$, event $_{j}$, state $_{r}, \ldots$ A simple path contains only one iteration of a loop, if a loop is present in some sequence."

For the coverage criterion, according to Binder's definition [22], "all-round-trip path coverage is obtained when every sequence of specified transitions beginning and ending in the same state is exercised at least once". To satisfy this coverage criterion, a test suite should contain all possible $\mathrm{k}$ transition sequences starting from any one state and ending at the same state where $\mathrm{k}$ is the enumeration of 1 to the number of total states. According to Binder, all-round-trip paths coverage is effective for detecting several important classes of defects, such as "incorrect/missing transitions, incorrect/missing actions, missing states, and some corrupt states" [23].

Binder presents a method to build up a round-trip-path transition tree. Based on Binder's methodology, different transition trees could be built up according to different searching sequences by the same searching method or by applying different searching methods. Briand et al. [5] researched some variant round-trip-path transition trees (built up according to different searching sequences by a breadth-first searching method), pointed out the difference of defect detection effectiveness between the variant transition trees (for example, some transition trees may miss some detectable defects), presented a modified transition tree and observed that the test suite derived from the modified transition tree is effective to detect defects.

We present Binder's method to build up a transition tree in section 3.1.3.1, and Briand et al.'s method to build up a modified transition tree in section 3.1.3.2. 


\subsubsection{Transition Tree}

The algorithm suggested by Binder for creating a round-trip paths adequate test set is based on the construction of a transition tree [22]. This criterion creates a test suite to cover all-round-trip paths in a piece-wise manner, which means each round-trip path is covered by combining parts of two or more test paths. For example, one round-trip path @stateA@transition1@stateB@transition2@stateC@transition3@stateA (where symbo1 (a) is used as a delimiter between states and transitions) is covered by two test paths: @stateA@transition1@stateB@transition2@stateC and@stateA-@transition4@stateC @transition3@stateA. We will refer to this criterion as the transition tree technique throughout the rest of this thesis.

According to Binder [22], the steps for deriving a transition tree from a state machine diagram are given as follows ${ }^{1}$ :

Step 1: The initial state of the state machine is defined as the root of the tree.

Step 2: If the current working state has appeared somewhere else in the tree or it is a final state, the corresponding node is marked as terminal. Otherwise, for each transition leaving the state, draw an edge marking the event, guard condition, and action, and draw a node for the state this transition will enter.

Step 3: Repeat step 2 until all nodes in this tree are marked as terminal.

The traversal of the state machine diagram to build the transition tree can follow a depthfirst or breadth-first algorithm. Either way, the resulting transition tree satisfies the allround-trip criterion.

When a transition in the state machine diagram has a guard condition, Binder suggests the following: if the guard condition is a simple predicate or a predicate with only AND operators, add one branch for that transition in the tree; if the guard condition is a

\footnotetext{
${ }^{1}$ Note that before the algorithm can be used (i.e., the steps followed), the state machine diagram has to be flattened; that is, the concurrency and composition aspects of the UML state machine diagram notation have to be removed. A procedure for flattening a state machine diagram is proposed in [24]. This aspect is beyond the scope of this thesis.
} 
complex predicate with OR operators, add a new branch for each truth value combination that is sufficient to make the guard true. For example, if a transition $t 1$ leading a SUT object from state $A$ to state $B$ has a guard condition $[a t t 1>0 \& \&$ att $2<0]$, there is only one test path from state $A$ to state B:@stateA@t1[att1>0\&\&att2<0]@stateB; if $t 1$ has a guard condition $[a t t 1>0 \| a t t 2<0]$, there are two test paths from state $A$ to state $B$ : @stateA@t1[att1>0 \&\& att2>=0]@stateB and @stateA@t1[att1<=0 \&\& att2<0]@stateB. Additionally, if a guard condition becomes true only after repeating some events, one edge in the tree is added, labeled with these events and appended with an asterisk $(*)$, showing the need for repetition.

After a transition tree is built, each transition tree path is transcribed to a test case. A test path in a transition tree is a sequence of transitions leading a SUT object from the initial state to a leaf state in this transition tree.

Figure 2 gives a brief example of a state machine diagram and the derived transition tree. In Figure 2, (A) shows a short state machine diagram, (B) shows a derived Binder's transition tree based on a depth-first search, and (C) shows a derived transition tree based on a breadth-first search.

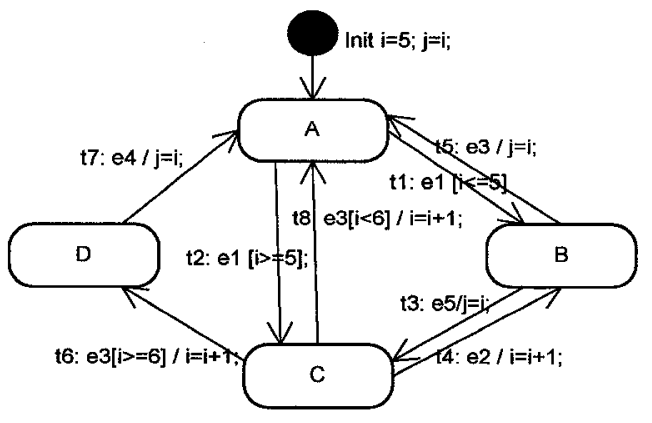

(A)

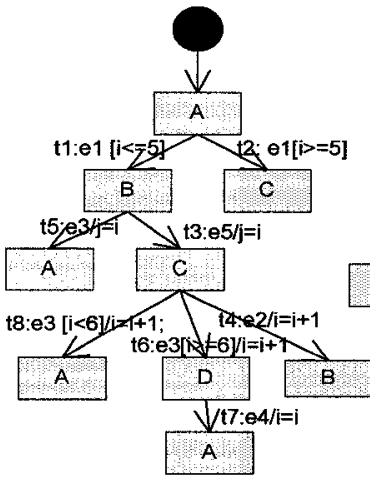

(B)

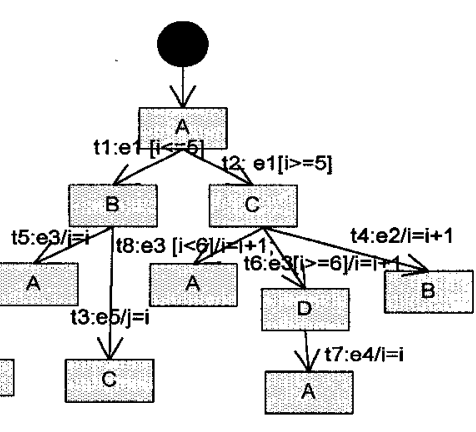

(C)

Figure 2: A simple state machine diagram (A) and the derived transition trees: by depth-first search (B), and by breadth-first search (C)

When Binder's depth-first transition tree is transcribed to a test suite, there are five test cases, as follows:

1. @A@ 
2. @A $A @ t 1[i<=5] @ B @ t 5 @ A ;$

3.1@A@t1[i<=5]@B@t3@C@t8[i<6]@A

4.1@A@t1[i<=5]@B@t3@C@t6[i>=6]@D@t7@A

5._@A@t1[i<=5]@B@t3@C@t4@B

The test case for test path (4) is not feasible because of guard conditions ( $i$ should satisfy $i<=5$ and $i>=6$ ). To satisfy the guard condition, a complementary transition sequence @C@t4@B@t3@C should be marked with an asterisk and inserted before transition t6, but it does not appear in this test path, or anywhere in the transition tree.

Based on Binder's algorithm for deriving a transition tree, a tester can derive several variant transition trees, using a depth-first search or breadth-first search. In [5], Briand et al. stated that these variant transition trees may have different defect detection capabilities.

\subsubsection{Modified Transition Tree}

A modified transition tree proposed by Briand et al. in [3] changes the termination criterion for building transition trees. In this criterion, a state node in a transition tree is marked as terminal if the state is a final state or the state has appeared in the path leading to that node. The generated transition tree ensures that each test path contains one complete round-trip path, whereas Binder's definition results in covering some round-trip paths entirely and some round-trip paths only in a piece-wise manner. An example is given in Figure 3, including a state machine diagram, Binder's transition tree and a modified transition tree. 


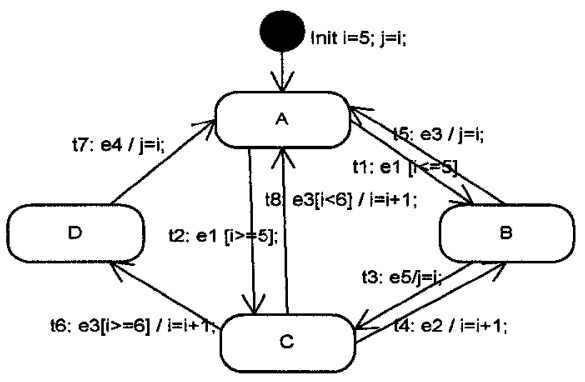

(A)

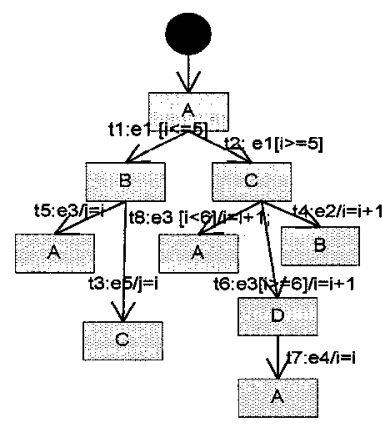

(B)

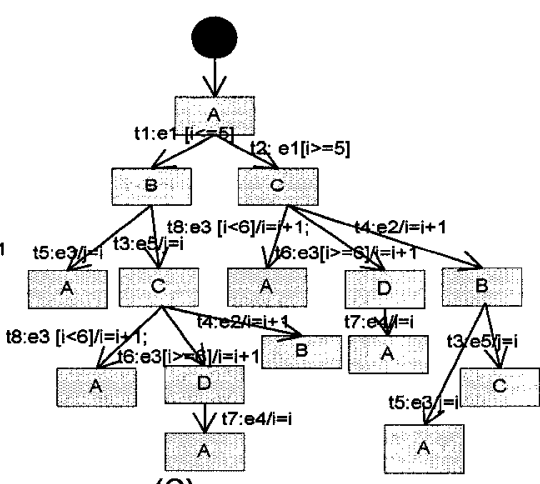

(C)

Figure 3: A state machine diagram (A), a transition tree derived following Binder's rule (B) and a modified transition tree (C).

This criterion leads to more expensive testing: transition tree (B) has more test paths than transition tree (A). In the same way as Binder's transition tree, to satisfy guard conditions, a complementary transition sequence should be derived manually.

Most of state-based testing works we discussed before, if not all of them, assume software testers have a getState method of the SUT or have access to attributes, and associations of the SUT. So software testers can check the state of the SUT at the end of every transition or test case. On the other hand, in the area of state-based protocol conformance testing, works assume the SUT to be a complete black box $[25,26]$. To ascertain the state of a SUT, the researchers have devised the notion of characterization set. The characterization set is supposed to be executed at the end of every test case and it is able to help tester ascertain the state without any getState method or any access to attribute values. Our work is based on an existing source code with a getState method and full access to attributes and associations of the SUT.

\subsection{Data Flow Information in Operation Contracts}

Operation contracts describe conditions and changes to a SUT object when an operation takes place. Operation contracts include conditions (on attributes, parameters, links) that a caller must satisfy before an operation is executed, namely pre-conditions, as well as changes (to attributes, parameters, links) after the operation is executed, namely postconditions, thereby revealing data flow potentially triggered during operations executions 
[27]. As an option in UML, operation contracts can be expressed using the Object Constraint Language.

Briand et al. [27] provided rules for identifying definitions and uses of model elements (e.g., attributes, associations) from operation contracts. This was particularly applied to UML state machine diagrams by analyzing operation pre- and postconditions (operations triggering events, actions resulting from triggered events), class invariants, as well as guard conditions, all specified in OCL. A transition sequence, and more generally a transition tree, can be transformed into an event/action flow graph (EAFG) that accounts for the control flow suggested in pre and post conditions. Finally, definitions and uses of model elements are identified from OCL constraints (guard conditions, terms in pre and post conditions) by a set of rules and the data flow information can be derived. Typically, model elements that appear in OCL constraints on edges of an EAFG are identified as puses, and model elements that appear in OCL constraints on nodes are identified as either c-uses or definitions (see [27] for further details). Then criteria such as all-definitions (i.e., for each definition, test cases must trigger a path from that definition to a use) and allDU-paths (i.e., for each definition and each reachable use, test cases must trigger all possible paths) can be used to define test cases. One important observation Briand et al. made on three different case studies (actually the three ones we will be using in Section 6) is that the set of DU-paths equals the set of DU-pairs (i.e., only one definition-clear path from the definition to the use for each DU-pair). It is therefore sufficient to determine DU-pairs to cover DU-paths on the three case studies. In this thesis, it is named as all-DU pairs. This may not be the case in general though, and only further studies will confirm (or not) this result. The authors also noticed that the data flow analysis they propose can be used as an indicator to reveal a test suite's capability in defect detection. A general rule is that in a set of variant transition trees based on one state machine diagram, the transition tree covering the most data flow information (in the model) has better capability for detecting defects.

We adopt the rules of Briand et al. [27] to identify definitions and uses of model elements from operation contracts. 
As noted by the authors of [28], a state machine diagram only shows the attributes that have an effect on the behavior of the SUT object, but does not show local attributes in the implementation. They therefore propose a grey-box (both based on the model and the code) state based testing approach whereby transition sequences are derived from the state based model and derive test case from an analysis of the data flow triggered in the code by these sequences. This method cannot be used in the analysis phase because local variables are not discussed in the analysis model and software testers have to wait for the code implementation to start their work.

\subsection{Related Work on Meta-heuristic Search for Software Testing}

Meta-heuristic search is a general concept for expressing a search algorithm; it is based on blind sampling and is heading in a promising direction. It encompasses a set of algorithms that have been successfully applied to solving combinatorial optimization problems. Besides genetic algorithms (GAs), the simulated annealing (SA) is another often-used algorithm. This algorithm allows well controlled movements in a search space, which probably go through worse areas temporarily and ultimately head to the optimal areas [29]. (Please refer to [30] for detailed information).

In the software testing field, many researchers have applied meta-heuristic algorithms to generate test data. Tracey et al. [29] applied the SA to dynamically generate test data for satisfying structural testing criteria, such as branch predicates coverage (test data to cover all branches), boundary value analysis (test data close to boundary), assert/run-time exception testing (test data to break assertions or cause run-time exceptions) and component re-use testing (test data to satisfy/break pre-condition of a re-usable component). Mansour et al. [31] applied the SA and the GA in data generation for path testing; they applied heuristic algorithms to generate test data to exercise executable paths in a program. They also compared the results presented by the two algorithms. They observed that SA tends to perform slightly better than the GA in terms of the number of executable paths it identifies, but the GA is faster than SA. Derderian et al. [32] applied the GA to generate feasible input data sequences for extended finite state machines and indicated that the GA found one feasible transition path for each possible path length, but 
the random generation algorithm did not. Guo et al. [33] applied the SA and the GA to generate multiple unique input sequences for FSM based testing; the author gave penalties (during fitness evaluation) to solutions that are similar to each other in genotype; the author found that there is no significant difference between the GA and the $\mathrm{SA}$, but the SA and the GA with penalties for similar solutions outperforms the GA without penalties. Lakhotia et al. [34] presented a multi-objective approach to searchbased test data generation for branch coverage and dynamic memory consumption and showed the multi-objective GA is suitable for their application.

Meta-heuristic search algorithms are not only used in test data generation, but also in some other applications such as detecting error-prone paths [35] and prioritizing test case sequences for regression testing [36] or for integration testing [37].

In our work, we apply the genetic algorithm but not other algorithms like simulated annealing, hill climbing, or tabu search because all of these algorithms only maintain one solution during the search process. On the other hand, a genetic algorithm maintains a pool of solutions allowing more exploitation and exploration of the search space.

\subsection{Related Work on Test Case Prioritization}

Our research problem, for scheduling test cases of a test suite in an order, bears some similarities with current research on test case prioritization in the context of regression testing, which is to prioritize test cases of a test pool to detect defects as early as possible. In the context of regression testing, those techniques are based on the analysis of the source code. However, our technique is based on the analysis model.

In the context of regression testing, given $T$, a test suite, $P T$, the set of permutations of $T$, and $f$, a function from $P T$ to the real number, the prioritization problem is to find $T^{\prime} \in P T$ such that $\left(\forall T^{\prime \prime}\right)\left(T^{\prime \prime} \in P T\right)\left(T^{\prime \prime} \neq T^{\prime}\right)\left[f\left(T^{\prime}\right) \geq f\left(T^{\prime \prime}\right)\right]$ [38]. Different prioritization criteria have been proposed [38]: prioritizing incremental coverage of statements, incremental coverage of branches, and incremental probability of exposing faults, etc. (Fault exposing probability is evaluated from mutation analysis.) Other criteria have been suggested by the same authors [39]. 
In 2005, Srikanth et al. [40] first applied test case prioritization based on requirements, considering four prioritization factors: customer-assigned priority, requirements volatility, implementation complexity and fault proneness. They also provided a prototype tool, PORT, which requires traceability among requirements, test cases, and test/field failures. It allows the software tester to get involved in software testing earlier, but the prioritization factors of customer-assigned priority, implementation complexity and fault proneness are quite subjective in the analysis phase.

In 2007, for regression testing, $\mathrm{Li}$ et al. [36] compared several search methods for test case prioritization based on branch/block/statement coverage rate: three greedy searching approaches, hill climbing, and the genetic algorithm to ascertain an optimal test sequence for regression testing. The authors mentioned that the empirical result shows the genetic algorithm performs well, although some of the greedy approaches are surprisingly effective. 


\section{TAILORED GENETIC ALGORITHM}

In this chapter, there are four sections. Section 4.1 discusses how the permutation problem of test cases is represented in the genetic algorithm. Section 4.2 discusses why this combinatorial optimization problem is a multi-objective problem, and how this problem can be solved using the multi-objective genetic algorithm. Section 4.3 discusses the fitness functions used to evaluate different objectives, and the combination of fitness functions based on different user expectations. Section 4.4 discusses the genetic algorithm's operators.

\subsection{Representation-Chromosome}

A chromosome is a sequence of test cases that is referred to as TCS in the remaining of this thesis. Any test suite satfisying the state-based criteria presented in Section 3.1 can be represented in this form.

First, we discuss a general method for chromosome representation and data flow information based on a state machine diagram. Since a test case may contain a sequence of transitions starting from any state in a state machine diagram, we use a test suite including test cases starting from any state in a state machine diagram as an example, in order to discuss how a TCS can be represented by a chromosome and how data flow information can be evaluated.

Since a test case contains a test path, which is comprised of a sequence of transitions, ultimately a chromosome is a long sequence of transitions that covers all test paths in all test cases. One issue that arises, which we refer to as the "executable issue", is that we can either consider that each test case (transitions sequence) in a chromosome is a separate sequence of transitions to execute (each possibly requiring a prefix sequence of transitions to bring the SUT in the right state from the start state) or consider that the whole chromosome is a sequence of transitions to execute (requiring one prefix sequence of transition to bring the SUT in the right state from the start state and then "bridging" sequences from the last state of each test case to the first state of the following test case, 
or identifying consecutive test sequences that overlap). In other words, we can consider that the chromosome is a sequence of test cases or one single test case. This leads to two different forms of chromosomes, which we discuss in section 4.1.1 and section 4.1.2. We then conclude in section 4.1.3.

\subsubsection{Form a Chromosome by Combining and Splicing All Test Paths}

In this approach, a chromosome is formed by combining and splicing all test paths. In this thesis, when we discuss combining and splicing test paths, we means that some overlapping transitions between test paths could be merged or some new transitions could be added to bridge test paths with the aim of creating one long executable transition sequence. Since test paths are combined and spliced, a test path is not suitable to be treated as a gene, which is the atomic element in the genetic algorithm. Instead a gene is a transition. At the same time, the genetic algorithm should ensure that the transition sequence of generated offspring is executable. The following example explains how test paths are combined and spliced.

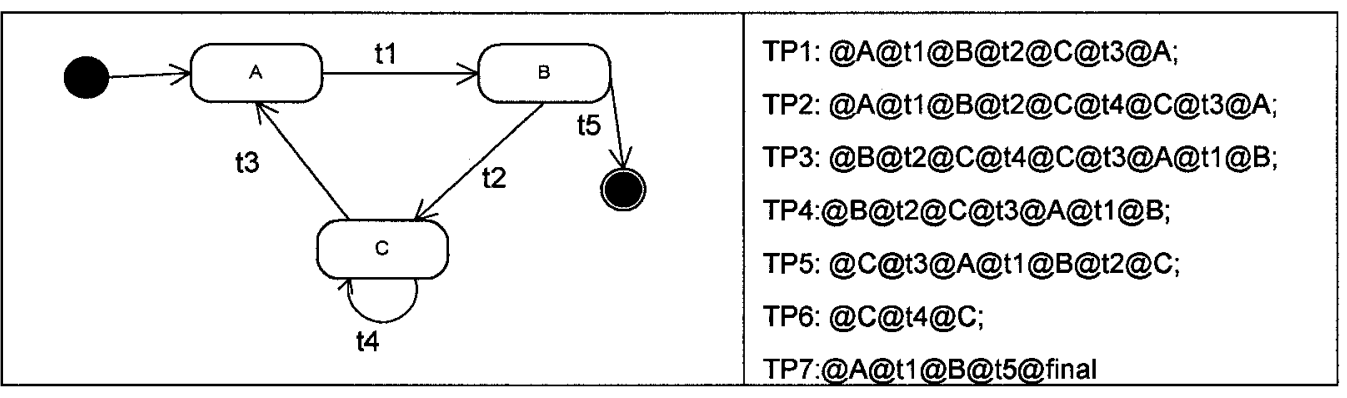

Figure 4: A simple state machine diagram and a test suite satisfying the all-roundtrip path criterion

Consider the state machine diagram in Figure 4 and seven test sequences TP1 to TP7. Assuming that one solution to the problem is to order TP1 after TP 3 in a chromosome, the formed transition sequence could be $@ B @ t 2 @ C @ t 4 @ C @ t 3 @ A @ t 1 @ B @ t 2 @ C @ t 3 @ A$ by identifying and merging the common transition sub-sequence of TP1 and TP3 (underlined in the chromosome above). Alternatively, the chromosome could be @B@t2@C@t4@C@t3@A@t1@B@t2@C@t3@A@t1@B@t2@C@t3@A by bridging test paths with extra transition sequence@t2@C@t3 (underlined in the chromosome 
above) so that the entire sequence of transitions become executable. (Special case: the final state could be considered equivalent to the initial state since a tester could reinitialize a SUT object to restart, so a test case ending with the final state could be followed by a test case starting with the initial state or its immediate successive state, e.g. the state $\mathrm{A}$ in the above example).

In this approach, a gene has to be a transition since test sequences can be concatenated or overlapping in a chromosome. With merging the common transition sequences or adding extra transition sequences, the different lengths of transition sequences give rise to variable-length chromosomes. For variable-length chromosomes, some particular GA crossover operator (e.g. cut $\&$ splice) and mutation operator (e.g. delete $\&$ insert) can be applied. On the other hand, the genetic algorithm (through crossover and mutation operators) needs to take care of the executable issue, ensuring that the whole transition sequences of offspring remains executable. This approach has the best potentiality to find the shortest transition sequence to cover all test paths, i.e., to reduce costs.

But this approach does not work if test paths contain guard conditions since then it is difficult, or even impossible to ensure a chromosome represents a single executable transition sequence. Consider for example the state machine diagram and test sequences in Figure 5. Transition sequence@A@t1@B@t2@C@t3@A@t1@B@t2@C@t4@C@ $13 @ A$ formed by connecting TP1 with TP2 may not satisfy the guard condition and the "executable issue" cannot easily (if ever) be solved.

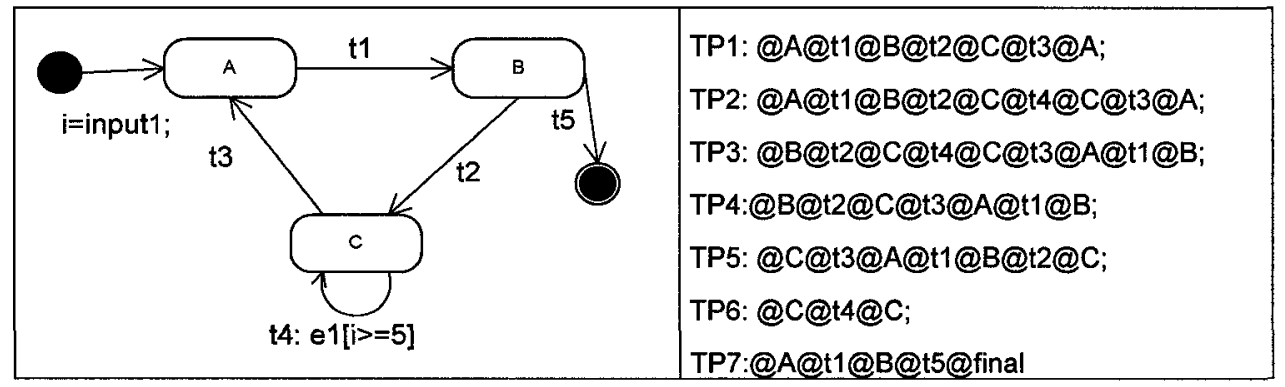

Figure 5: A state machine diagram with a guard condition and a test suite

As a result, this approach is not suitable for a state machine diagram with guard conditions. But for a rare state machine diagram without guard conditions, it may be useful and effective. 


\subsubsection{Form a Chromosome by Ordering All Test Paths}

In this approach, a chromosome is formed by ordering all test paths without considering combining or splicing them. Though the formed chromosome is per se a long sequence of transitions, each test case is considered separately (no attempt is made by the GA to move from one test path to its successor smoothly since the predecessor's last state probably doesn't match the successor's first state).

In this approach, a gene in a chromosome is a test path. The number of test paths in a test suite does not change, once set, since each chromosome contains all the test paths, so we have fixed-length chromosomes in the GA. The crossover operators such as PMX (partial mapped crossover), and the mutation crossover operators such as inversion, insertion, and reciprocal, could then be applied (see Section 4.4.2).

Once again, in this approach, we only simply order test paths but not combine or splice test paths. Based on the example in Figure 4, a formed transition sequence can be @A@t1@B@t2@B@t3@A-@A@t1@B@t2@C@t4@C@t3@A@B@t2@C@t3@A@t1@B-@B@t2@C@t4@C@t3@A@t1@B@C@t3@A@t1@B@t2@C-@C@t4@C@t3@A@t1@B@t2@C@A@t1@B@t5@final,(where ‘-'separates test paths.)

It is obvious that this sequence of transitions is not executable since the first states of the third, fifth and seventh test paths do not match their predecessor's last states. To solve this problem, we could apply a connection method to bridge these test paths. This would however be outside the scope of the GA. Three possible methods can be considered:

1. Identify the shortest transition sequence between the last state of the predecessor test path and the first state of the successive test path, then add all the shortest transition sequences to bridge all test paths. Thus the test path sequence is executable. The shortest test paths between any two states could be achieved by applying a graph algorithm to find the shortest path between any two vertices. The presence of guard conditions still remains a challenge for this purely graph-based solution. 
2. For each test path, have a manually developed "setState" method to set the state of the SUT object to the first state of that test path, and set the parameters, attributes or properties of navigated association to the right values for guaranteeing each test path is executable. This may require a lot of development work. The benefit of this method is that it does not increase the execution time to execute all test paths since it never adds any extra transitions to connect test paths.

3. For each test path, re-initialize the SUT object and prefix a transition sequence that takes the SUT object from its initial state to the first state of the path and satisfies guard conditions of that test path. Some development work is required to find a sequence from the initial state of the SUT to the first state of the test path.

\subsubsection{Summary of Methodologies}

Based on the above discussion about the GA presentation and the pros and cons for each method, we conclude with a comparison summary in Table 1. The two GA representations, fixed length chromosome or variable length chromosome, are evaluated according to eight different criteria: what a gene represents (transition or transition test sequence), possible GA operators, how to generate executable test sequences, whether the representation allows us to (easily) handle guard conditions, characteristics of state machines that are suitable for the representations. The table concludes with the characteristics of the test suite (and therefore state machine) that would best work with the representation.

As we want to develop an approach that is as general as possible, and therefore handle state machines with guard conditions, we will use fixed length chromosomes. 


\begin{tabular}{|c|c|c|c|c|}
\hline & \multicolumn{4}{|l|}{ GA representation } \\
\hline & $\begin{array}{l}\text { Variable length } \\
\text { chromosome }\end{array}$ & \multicolumn{3}{|c|}{ Fixed length chromosome } \\
\hline Gene & Transition & \multicolumn{3}{|c|}{ Transition test sequence } \\
\hline GA operator & $\begin{array}{l}\text { Crossover: Cut \& Splice } \\
\text { Mutation : Insert / Delete }\end{array}$ & \multicolumn{3}{|c|}{$\begin{array}{l}\text { Crossover : PMX } \\
\text { Mutation : Insert / Inverse }\end{array}$} \\
\hline $\begin{array}{l}\text { Executable } \\
\text { transition } \\
\text { sequence }\end{array}$ & $\begin{array}{l}\text { Merge common transition } \\
\text { sequence and splice test } \\
\text { paths by adding extra } \\
\text { transitions }\end{array}$ & $\begin{array}{l}\text { Add the shortest } \\
\text { transition sequence } \\
\text { between test paths. }\end{array}$ & $\begin{array}{l}\text { Develop "setState" } \\
\text { operation for each } \\
\text { test path }\end{array}$ & $\begin{array}{l}\text { Re-initialize the } \\
\text { SUT object and use } \\
\text { a prefix transition } \\
\text { sequence for each } \\
\text { test path }\end{array}$ \\
\hline $\begin{array}{l}\text { Handles guard } \\
\text { conditions }\end{array}$ & No & No & Yes & Yes \\
\hline $\begin{array}{l}\text { Suitable state } \\
\text { machine } \\
\text { diagram }\end{array}$ & $\begin{array}{l}\text { State machine diagram } \\
\text { without guard conditions }\end{array}$ & $\begin{array}{l}\text { State machine } \\
\text { diagram without } \\
\text { guard conditions }\end{array}$ & Any & Any \\
\hline $\begin{array}{l}\text { The best fit } \\
\text { test suite }\end{array}$ & $\begin{array}{l}\text { A test suite without any } \\
\text { guard condition }\end{array}$ & $\begin{array}{l}\text { A test suite without } \\
\text { any guard condition }\end{array}$ & $\begin{array}{l}\text { A test suite with test } \\
\text { paths starting from } \\
\text { all states. }\end{array}$ & $\begin{array}{l}\text { A test suite with test } \\
\text { paths starting from } \\
\text { one state. }\end{array}$ \\
\hline
\end{tabular}

Table 1: Summary of different GA representations

\subsubsection{Chromosome Properties}

In our problem, a chromosome has two important properties: one is the test cost (Section 4.1.4.1); the other is the cumulative data flow coverage rate (Section 4.1.4.2).

\subsubsection{Test Cost}

According to Binder [22], the cost of state-based testing comprises two parts: time and resource for each test case and for each test message (event) used in a test case. The cost of design, setup and post-test for each test case usually does not depend on the number of events in test cases. Since there is no general way to quantify this sort of cost and this sort of cost usually has no proportionally relationship with the number of events in test cases, we simply assume each test cost taking equivalent cost for design, setup and post-test and exclude them when we consider the test cost property of a chromosome. The cost per message includes the time and computer resource of storage, execution of the message and development of the message. It usually increases proportionally with the number of test messages. The cost per message could clearly identify the cost difference between 
test cases. A test case containing a longer transition sequence will take more cost to trigger more events, traverse more states and cover more behaviours than a test case containing a shorter transition sequence. As a result, we define the test cost of a test case as the number of transitions the test case contains. The test cost of any transition is treated equivalently.

Other, more refined measures of cost could be investigated but this measure of cost is currently the most widely used in the testing community and past empirical studies (e.g., [3]).

\subsubsection{Cumulative Data Flow Coverage Rate}

We rely on the methodology presented in [5], based on the analysis of OCL contracts, for identifying data flow exercised by sequences of transitions. We consider two criteria, namely all-definitions and all-DU-paths. (Recall from Section 3.2 that all-DU pairs has been shown to be equivalent to all-DU-paths.) We therefore compute two different data flow coverage rates - one for each criterion, that is the numbers of already covered definitions/DU-pairs divided by the total number of definitions/DU-pairs we can identify in the model. The number of already covered definitions/DU-pairs at a given transition $i$ is the total number of non-duplicate definitions/DU-pairs covered from the first transition to transition $i$. The cumulative data flow coverage rates at any transition $i$ are therefore defined as:

$$
\begin{gathered}
c_{\text {all-defs }}(i)=\frac{\text { TheNumberOfAlreadyCoveredDefinitionsAtTheTransition }(i)}{\text { TheTotalNumberOfDefinitions }} ; \\
c_{\text {all }- \text { uses }}(i)=\frac{\text { TheNumberOfAlreadyCoveredUsesAtTheTransition }(i)}{\text { TheTotalNumberOfDUPairs }} ;
\end{gathered}
$$

where the numerators are the number of data flow elements (definitions and uses) covered in the test case sequence up to test case number $i$, and the denominators are total numbers of definitions/DU pairs that can be covered by the whole test suite. (There may be definitions and uses in the model that are not covered by the whole test suite. These are not considered here.) In the remaining part of the thesis, the cumulative data flow 
coverage rate is often briefly called the cumulative coverage rate, and when the data flow elements of interest is not relevant we simply write $c(i)$ to denote a cumulative coverage rate.

\subsection{Multi-objective Optimization Problem}

Recall that our problem is to execute test cases in such an order that coverage of data flow is maximized as quickly as possible (since covering data flow has shown to relate to fault detection) while minimizing the cost of executing those test cases. For a chromosome, we therefore expect it increases its cumulative coverage rate as quickly as possible, finally reaching a certain data flow coverage rate, while minimizing its cost.

For a known set of test cases, the chart in Figure 6 shows the relationship of the cumulative coverage rate to the test cost for two chromosomes, i.e., two sequences of test cases (an optimal sequence and a sub-optimal sequence). Recall we assume that transitions have the same cost of one cost unit. Therefore, the cumulative coverage rate $\mathrm{Y}$ at test cost $\mathrm{X}$ is the cumulative coverage rate $\mathrm{Y}$ reached after the first $\mathrm{X}$ transitions have been executed. In our problem, the cumulative coverage rate is a series of discrete values. In the remaining sections, to easily show the relationship between the test cost and the cumulative coverage rate of a solution in a figure, we use a curve to connect its discrete values of cumulative coverage rate.

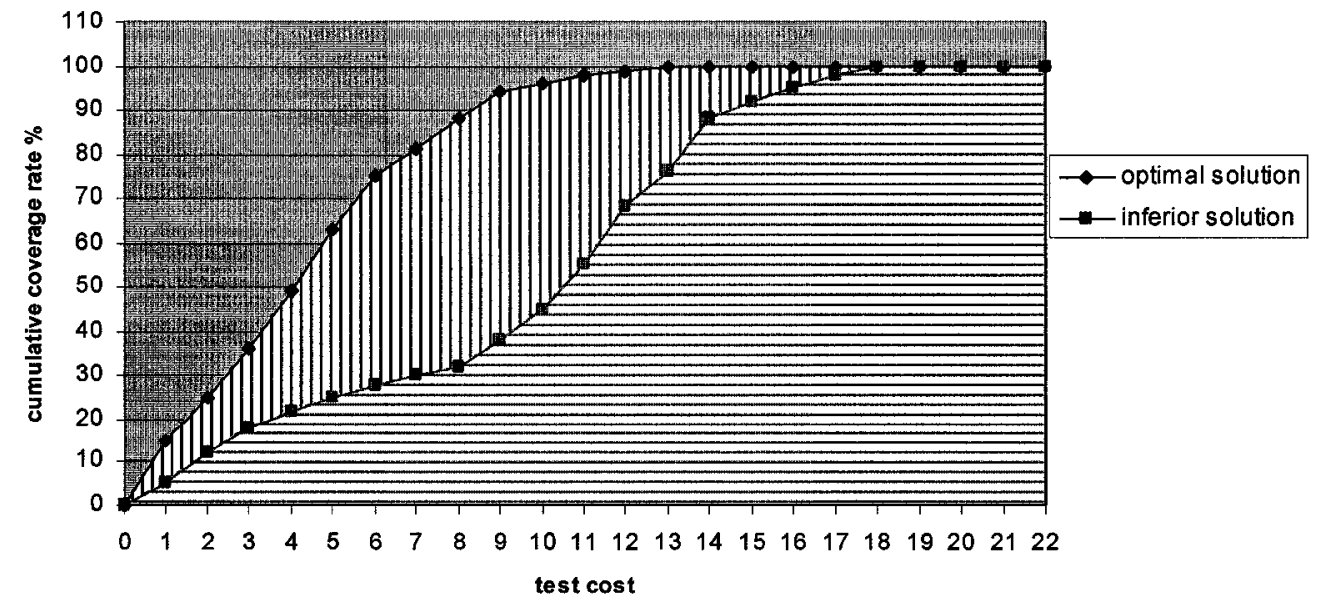

Figure 6: The cumulative coverage rate tendency of two solutions 
We could interpret Figure 6 as follows: the superior (resp. inferior) solution covers 25\% (resp. 12\%) of the data flow information when the first two transitions are executed, and $81 \%$ (resp. $30 \%$ ) of the data flow information when the first seven transitions are executed.

In practice, a user may expect a data flow coverage rate or have limited budget to execute test cases. Then the expected data flow coverage rate or the affordable budget are user constraints. In general, whatever the user constraints, we expect a good solution to increase its cumulative coverage rate sharply as soon as possible, as this would hopefully tend to help a user detect faults earlier during the testing activity. As well, we expect a good solution to reach the user specified data flow coverage rate as early as possible if it is given, or obtain a data flow coverage rate as high as possible at a certain cost if an affordable budget is given. The different solutions satisfying different expectations, the best solution with the data flow coverage rate increment or the best solution satisfying the user constraint(s), will be presented to the user for his/her own appraisal since only the test engineer has the expertise to decide which solution is best.

This general problem can be decomposed in at least four different sub-problems, that we refer to as schemes, depending on the requirements of the test engineers, as discussed in the following sections: the test engineer has a budget and wants to maximize coverage (section 4.2.1), the test engineers decides to only achieve a maximum coverage rate and reduce costs (section 4.2.2), the test engineer wants to achieve full coverage and reduce costs (section 4.2.3), the test engineer specifies a minimum coverage to be reached and a maximum cost allowed (section 4.2.4).

\subsubsection{Optimization Scheme 1-Specifying a Maximum Test Cost}

If a test engineer has a given budget for a testing campaign, the available execution time is estimated to allow him/her to execute a part of the test cases with the maximum of $\mathrm{X}$ transitions rather than all test cases with the total number of transitions. The engineer will want to maximize coverage within the cost budget. 
One objective is therefore to maximize the sum of cumulative coverage rates within the given test cost, i.e., the area defined by the curve of cumulative coverage rates, as illustrated in Figure 6.

However, it is possible that the available test cost is too small for any test case subset arranged in a specific order (to maximize early coverage) to achieve maximum coverage $(100 \%)$ while staying within the allowed budget. In such a situation, the solution with the best sum of cumulative coverage rates may not possess the best cumulative coverage rate at the given (budget) test cost. In Figure 7, assuming the budget is 9 cost units, solution 1 has a larger sum of cumulative coverage rates (area under the curve) than solution two, but solution 2 has a better overall cumulative coverage rate (close to 90\%). In such a situation, we tend to keep both solutions, since only the test engineer has the expertise to decide which solution is best.

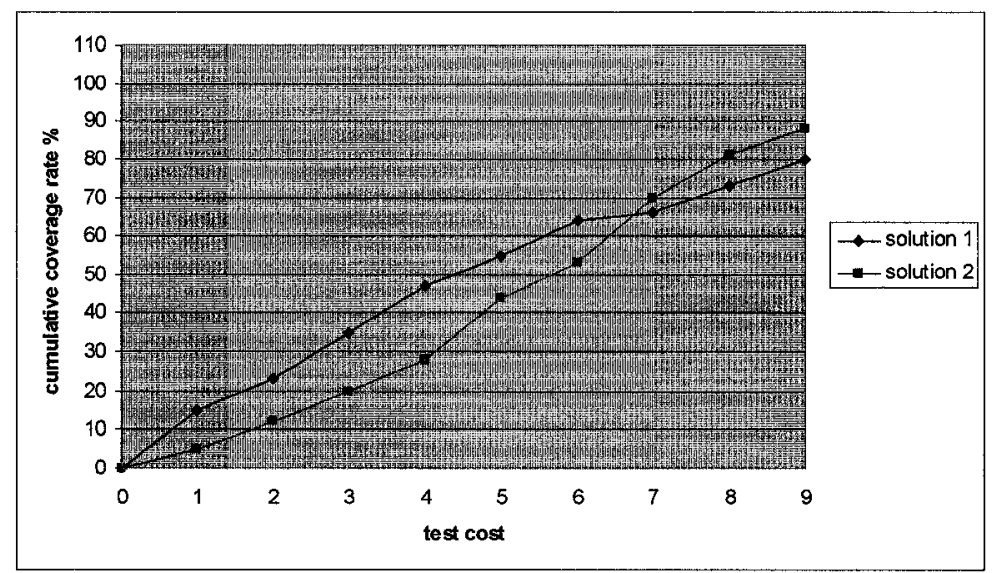

Figure 7: With a given test cost, an example with two possible solutions

Thus, in this scheme, there are two objectives:

1. Maximize the sum of the cumulative coverage rates within the given test cost.

2. Maximize the cumulative coverage rate at the given (budget) test cost.

\subsubsection{Optimization Scheme 2-Specifying a Cumulative Coverage Rate}

A test engineer may decide to cover a certain amount of data flow information to gain enough confidence in the SUT, while minimizing costs. 
To satisfy this requirement, we expect solutions to cover more data flow information at the beginning of the transition sequence and to quickly reach the required cumulative coverage rate as early as possible. However, assuming the specified cumulative rate is $75 \%$ for the solutions of Figure 7, solution 2 reaches the data flow coverage rate first but its cumulative coverage rate before the sixth transition is worse than solution 1 . In such a situation, we tend to keep both solutions, since only the test engineer has the expertise to decide which solution is best.

In this scheme we only consider the cumulative coverage rate value before the given cumulative coverage rate is reached, so we stop evaluating the cumulative coverage rate once a solution reaches the given value. For the two solutions of Figure 7, we calculate the area bounded by the y-axis, the curve and the straight (horizontal) line of the expected cumulative coverage rate, which is shown as the shadowed area in Figure 8. A solution with a greater sum of cumulative coverage rates, such as solution 1 , has a smaller value of the shadowed area.
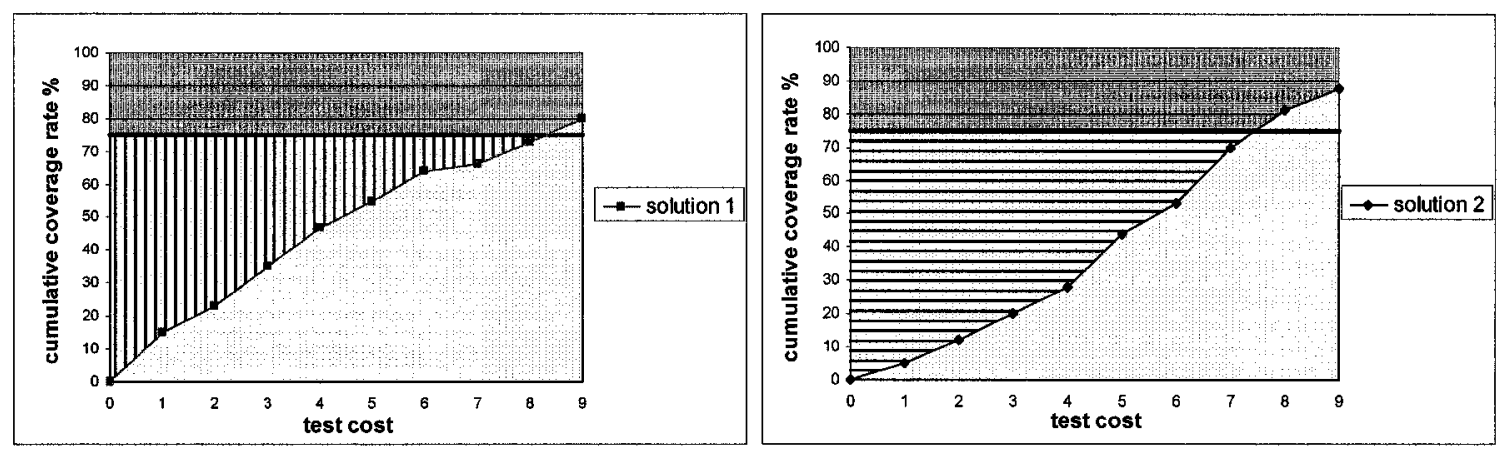

Figure 8: An example of two possible solutions reaching the given cumulative coverage rate of $75 \%$ with slightly different test costs.

Note that minimizing the area above the curve is not equivalent to maximizing the area below the curve, especially when solutions are very different, as illustrated in Figure 9 (assuming the specified cumulative coverage is .75\%): solution 1 has coverage rates.5, .6 and .8 at costs 1,2 , and 3 , whereas solution 2 has coverage rates $.1, .2, .3, .4, .5, .6, .7$, and .8 at costs 1 to 8 . In this case, maximizing the area below the curve (i.e., between the $\mathrm{x}$-axis, the curve and the vertical line at which the curve reaches the specified cumulative coverage rate) would promote solution 2 (are of 3.6) rather than solution 1 (area of 1.9). 


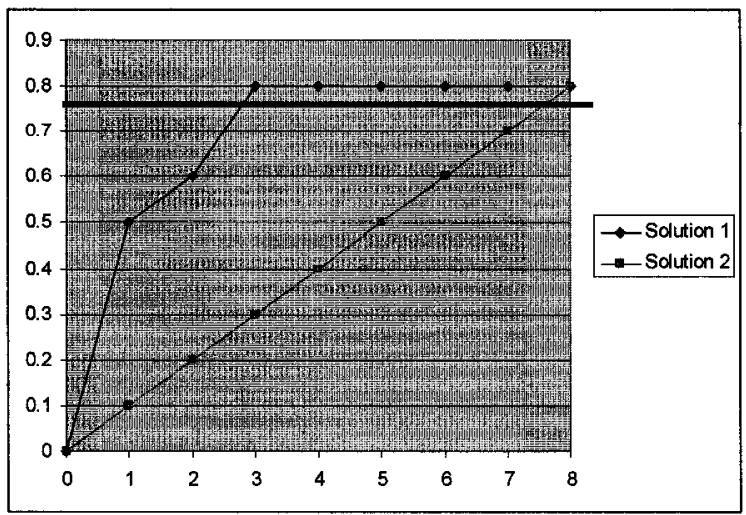

Figure 9: An example of two possible solutions reaching the given cumulative coverage rate of $75 \%$ with very different test costs.

Thus, in this scheme, there are two objectives:

1. Minimize the area surrounded by the solution's cumulative coverage rate curve, the $y$-axis and the straight (horizontal) line defined by the given cumulative coverage rate;

2. Minimize the test cost to reach the given cumulative coverage rate.

\subsubsection{Optimization Scheme 3-Specifying $100 \%$ Cumulative Coverage Rate and Maximum Available Test Cost}

A test engineer may be required to cover all data flow information (100\% coverage) regardless of test cost, so the (maximum) available budget (or test cost) can be the total number of transitions.

In this optimization scheme, different solutions may cover $100 \%$ of data flow information before all transitions are executed. In addition to minimizing costs, the engineer may be interested in solutions that cover more data flow information at the beginning of the transition sequence and quickly reach $100 \%$ of the cumulative coverage rate as early as possible. For the example in Figure 10, solution 1 which increases its data flow coverage rate quickly at the beginning of the transition sequence has a greater sum of cumulative coverage rates than solution 2 . Solution 2 , which increases its data flow coverage rate sharply in the middle of transition sequence, reaches $100 \%$ of cumulative coverage rate 
with a smaller test cost than solution 1. In such a situation, we tend to keep both solutions, since only the engineer has the expertise to decide which solution is best.

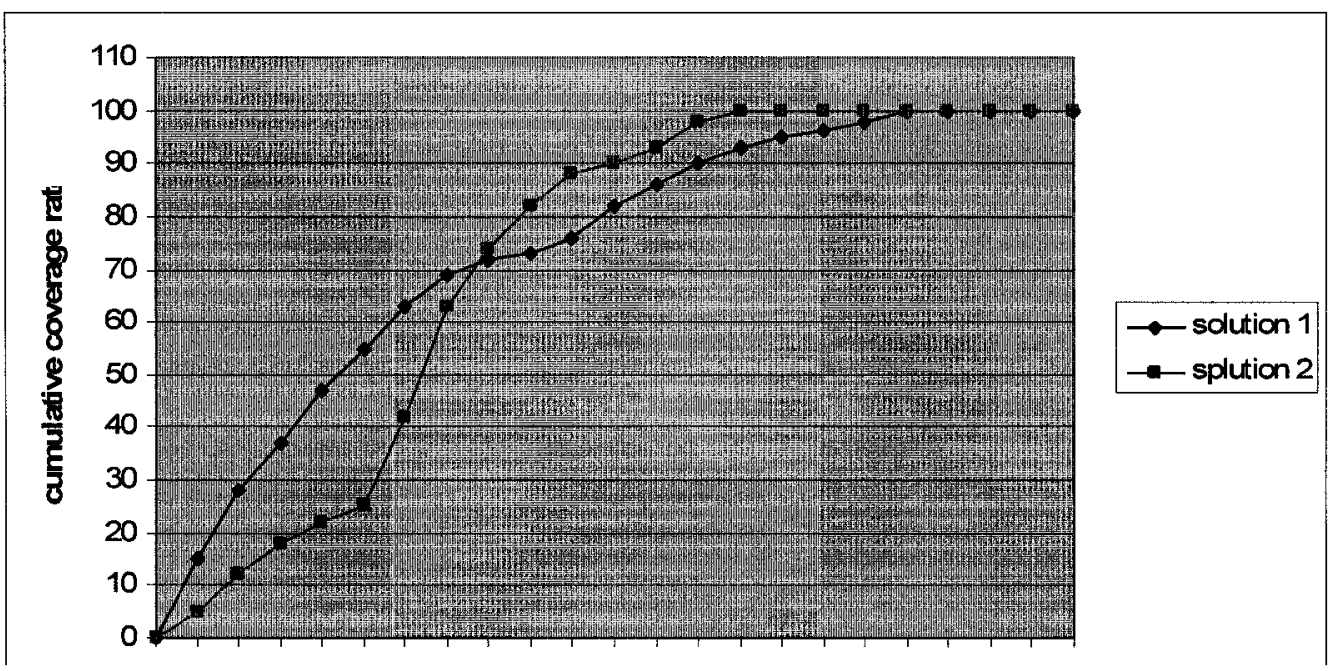

Figure 10: With a $100 \%$ cumulative coverage rate; an example with two possible solutions.

Thus, in this scheme, there are two objectives:

1. Maximize the sum of the cumulative coverage rates when accounting for all the transitions;

2. Minimize the test cost to reach $100 \%$ of the cumulative coverage rate.

\subsubsection{Optimization Scheme 4-Specifying a Maximum Test Cost and a Minimum Cumulative Coverage Rate}

A test engineer may have a limited budget and want to have enough confidence in the SUT by specifying a minimum coverage rate to achieve. With these two requirements, there are three possible situations:

1. The specified budget is only enough to achieve the expected coverage for some subsets of test cases but not for all possible subsets of test cases;

2. The specified budget is so large that any test case sequence can achieve $100 \%$ of data flow coverage (or the specified minimum coverage rate); 
3. For any subset of test cases, the expected coverage is too high to be achieved within the specified budget.

Figure 11 shows an example of the first situation, assuming the specified budget is 20 and the specified coverage rate is $80 \%$.

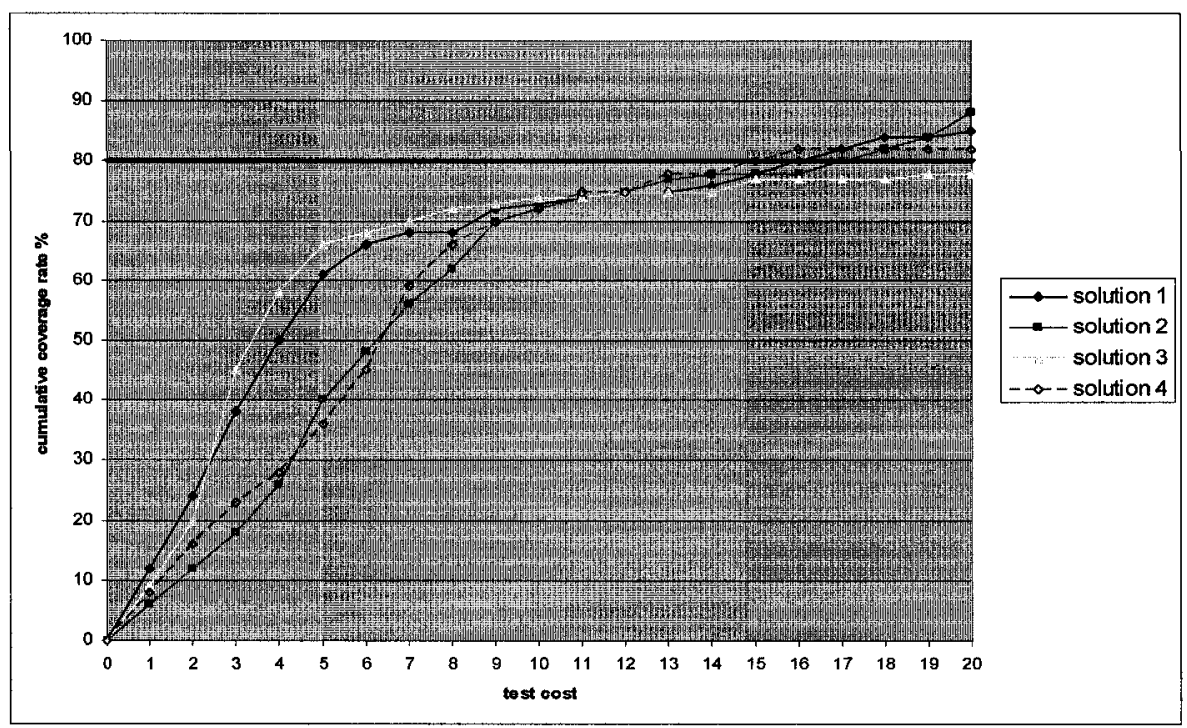

Figure 11: Four possible solutions in optimization scheme 4

Solution 1 has the biggest sum of cumulative coverage rate. Solution 2 has the best cumulative coverage rate at the given test cost 20 . Solution 3 has the smallest area bounded by the y-axis, the cumulative coverage rate curve and the horizontal straight line of expected cumulative coverage rate $80 \%$. Solution 4 has the shortest test cost at which its cumulative coverage rate reaches the expected cumulative coverage rate of $80 \%$. In this case, we tend to keep all solutions since only the engineer has the expertise to decide which solution is best.

Therefore, we have the following objectives:

1. Maximize the sum of the cumulative coverage rates within the specified test cost.

2. Maximize the cumulative coverage rate at the specified test cost.

3. Minimize the area bounded by the solution's curve, the y-axis and the straight (horizontal) line of the specified cumulative coverage rate. 
4. Minimize the test cost to reach the specified cumulative coverage rate.

Using these four objectives, the test engineer will be able to see useful results for each of the three situations mentioned above: the solutions that can achieve the specified coverage within budget (situation 1), the solutions that can achieve the specified coverage with a smaller budget than the one specified (situation 2), the solutions that maximize coverage (though smaller than the one expected) within the specified budget (situation 3 ).

\subsection{Objective Functions}

In section 4.2, we discussed the fact that the problem is multi-objective, and we discussed objectives of several optimization schemes. This section formalizes the objectives in terms of objective functions and discusses how these objective functions work together to find heuristic solutions in each optimization scheme. As illustrated in section 4.2, the different schemes rely on the same optimization criteria, which we formalize in section 4.3.1. Sections 4.3.2 to 4.3.5 then show how this formalization is used in the different schemes.

\subsubsection{Objective Functions}

\section{Sum of cumulative coverage rates}

The sum of cumulative coverage rates can be visualized ${ }^{2}$ as illustrated by the shadowed area in Figure 12.

\footnotetext{
${ }^{2}$ Note that the shadowed area is simply used here to visualize the objective function and provide intuition to the reader. Though it would be feasible, we do not want to suggest that we compute the size of the area (see footnote 3 ).
} 


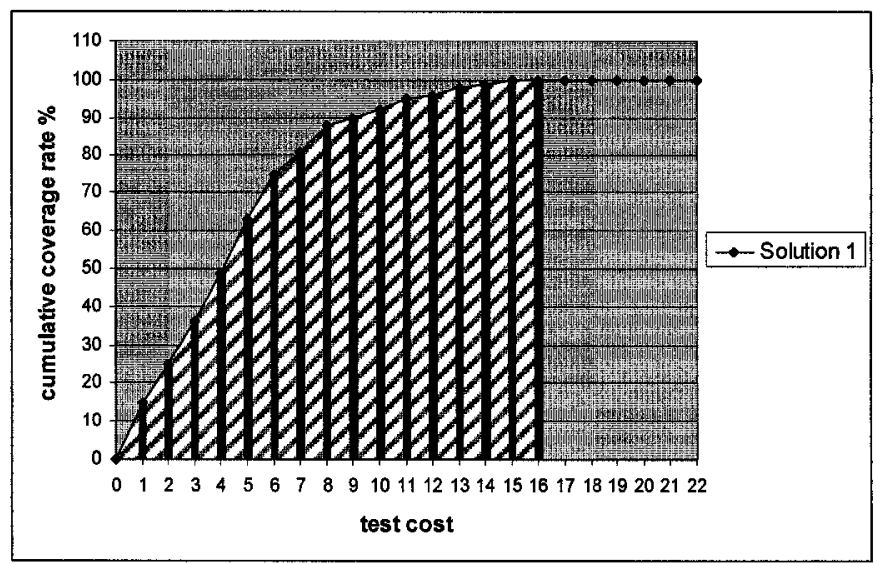

Figure 12: Maximize the sum of cumulative coverage rates with a given test cost 16.

Since we are interested in sharp increases early in the sequence of test cases, we multiply the cumulative coverage rate at each test cost value with a weight factor. To avoid bias (e.g., by defining those weights a priori), the weight factor is directly related to test cost: The weights linearly decrease as the cost increases. The objective function for the sum of the cumulative coverage rates is therefore ${ }^{3}$ :

$f_{1}(i): \operatorname{SumCumulCov}(i)=\sum_{j=1}^{j=T}(T-j+1) * c(i, j)$;

where $T$ is the maximum test cost value (on the $\mathrm{x}$ axis) used to compute the area ( $T$ is smaller or equal to the total number of transitions in the test suite we prioritize), and $c(i, j)$ denotes the cumulative coverage rate at a test cost $j$ for chromosome $i$, i.e., $c(j)$ of chromosome $i$ as we defined it in section 4.1.4.2.

\section{Cumulative coverage rate at a specified test cost}

With a specified test cost, the cumulative coverage rate at that test cost $T$ is simply: $f_{2}(i): \operatorname{CumulCov} \operatorname{Cost}(i)=c(i, T)$.

\footnotetext{
${ }^{3}$ An alternative can be to extrapolate the curve, for instance with $3^{\text {rd }}$ degree splines, and compute the integral of the splines. We considered that our sum, though an approximation of the area under the curve, would be a small approximation (especially since the $\mathrm{x}$-axis is split into equal segments of value 1 , as we assume the cost of a transition to be one) and would be more efficient (the GA will likely perform thousands of evaluations of this function).
} 


\section{Sum of differences between cumulative coverage rate and a specified coverage rate}

One of the objectives mentioned in section 4.2 is to minimize the area bounded by the solution's curve, the y-axis and the straight (horizontal) line of a given cumulative coverage rate (referred to as SC), as illustrated by the shadowed area in Figure 13.

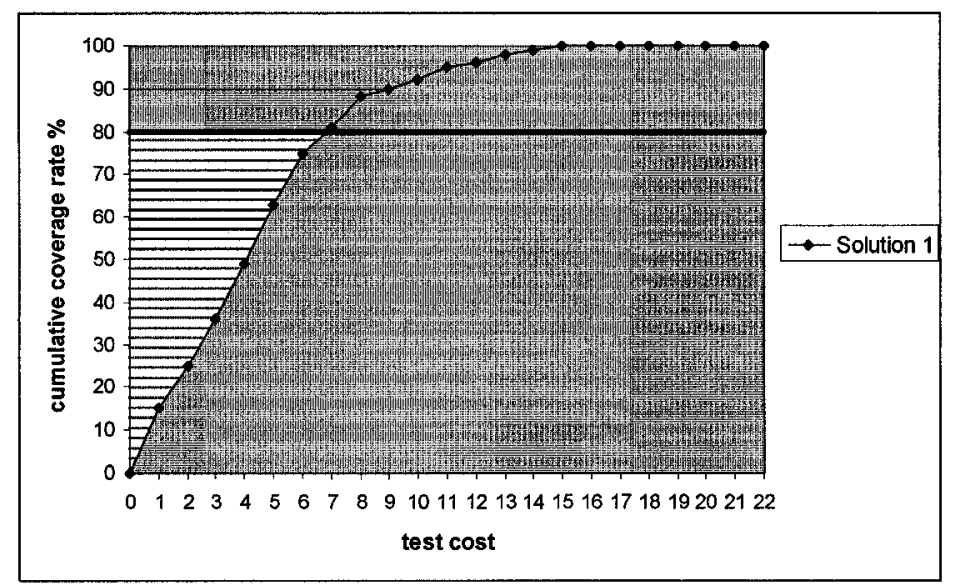

Figure 13: Minimize the shadowed area with a given cumulative coverage rate $80 \%$.

To estimate ${ }^{2,3}$ the area above the curve, we can add all differences between the cumulative coverage rates and the specified cumulative coverage rate ( $80 \%$ in Figure 13 ) at all test costs from test cost 0 to test cost $\bar{T}-1$ (where $\bar{T}$ is the first test cost where the cumulative coverage rate reaches/exceeds the specified cumulative coverage rate $S C$, and therefore $\bar{T}-1$ is the last test cost where the cumulative coverage rate does not reach the specified cumulative coverage rate). Since we are interested in sharp increases early in the sequence of test cases, similarly to fitness function $f_{1}(i): \operatorname{SumCumulCov}(i)$, we multiply the cumulative coverage rate at each test cost value with a weight factor, which is directly related to test cost: The weights linearly decrease as the cost increases. The objective function is:

$$
f_{3}(i): \operatorname{SumDifCov}(i)=\sum_{j=1}^{j=\bar{T}-1}(T-j+1) *(S C-c(i, j)) ;
$$

where $T$ is the user specified test cost (if $T$ is not specified such as in optimization scheme 2 , it is the default test cost - i.e., the total number of transitions), $S C$ is the specified 
cumulative coverage rate and $\bar{T}$ denotes the first test cost at which the cumulative coverage rate reaches/exceeds the given cumulative coverage rate $S C$. In other words:

$\bar{T}=l \mid c(i, l-1)<S C \wedge c(i, l) \geq S C$.

\section{Test cost for a specified cumulative coverage rate}

This objective function represents the test cost for a specified cumulative coverage rate, i.e.:

$f_{4}(i):$ CostAtRate $(i)=\bar{T}$;

where $\bar{T}=l \mid c(i, l-1)<S C \wedge c(i, l) \geq S C(\bar{T}$ is the first test cost at which the data flow cumulative coverage rate reaches/exceeds the user specified cumulative coverage rate $S C)$.

\subsubsection{Optimization Scheme 1 -Specifying a Maximum Test Cost ${ }^{4}$}

A test engineer specifies an expected test cost, less than the total number of transitions in the test suite, with a default cumulative coverage rate of $100 \%$. The optimization problem is therefore:

$\max : f_{1}(i): \operatorname{SumCumulCov}(i)=\sum_{j=1}^{j=T}(T-j+1) * c(i, j)$

$\max : f_{2}(i):$ CumulCovCost $(i)=c(i, T)$.

\footnotetext{
${ }^{4}$ In sections 4.3 .2 to 4.3 .5 , we reuse the notation of section 4.3.1. $T$ is therefore the user-specified maximum test cost allowed and $S C$ is the user-specified minimum cumulative coverage rate to reach.
} 


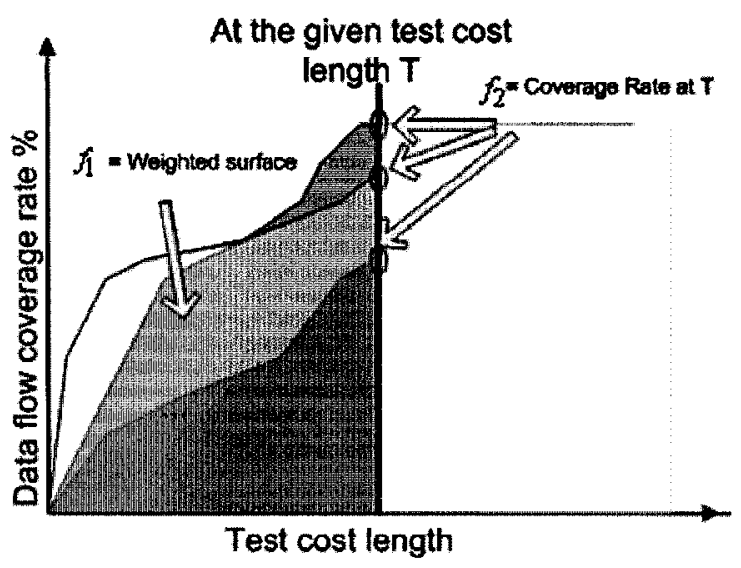

(a)

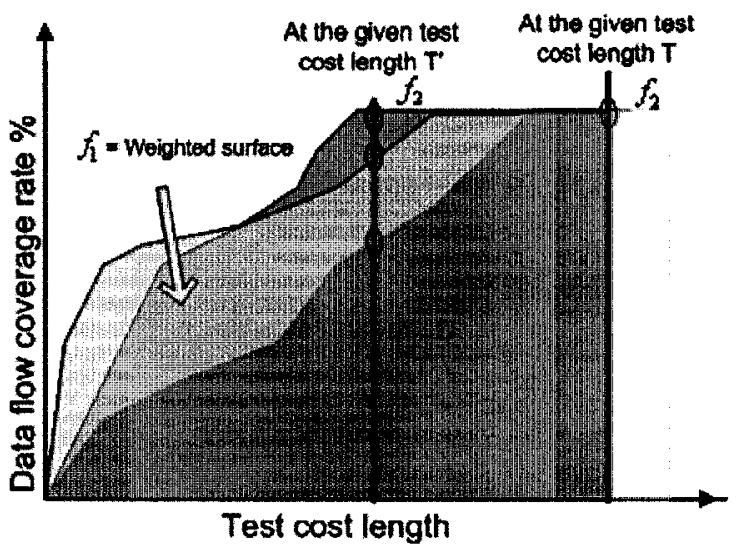

(b)

Figure 14: Optimization scheme 1 fitness functions-examples

Figure 14 (a) illustrates this scheme using three chromosomes, i.e., using three coverage rate curves. The objective function $f_{1}(i): \operatorname{SumCumulCov}(i)$ is the weighted area, bounded by the curve, the $\mathrm{x}$-axis and the vertical line at $\mathrm{x}=T$. The objective function $f_{2}(i)$ : CumulCovCost $(i)$ is the cumulative coverage rate at $T$, as indicated by ovals. From the above objective functions, the chromosomes with the best weighted area and with the best cumulative coverage rate at test cost length $T$ will be kept as non-dominated solutions by the objective functions $f_{1}(i)$ : SumCumulCov(i) and $f_{2}(i)$ : CumulCovCost(i).

If the given test cost $T$ is too large, all solutions in a population may reach $100 \%$ cumulative coverage rate before $T$ as illustrated in Figure 14 (b). All solutions then have the same fitness value for $f_{2}(i): \operatorname{CumulCov} \operatorname{Cost}(i)$, i.e., 100\%. The multi-objective genetic algorithm becomes a single objective genetic algorithm since the objective function $f_{2}(i)$ : CumulCovCost $(i)$ does not contribute to the SPEA2 fitness functions any more. We can then stop the GA search since good solutions have been found: the GA can return the solutions in the archive as they achieve $100 \%$ cumulative coverage with lower costs than the one expected (and specified) by the test engineers. Alternatively, we can let the GA find even better solutions. However, to increase the competition between solutions by relying on two fitness functions, we have to change the maximum test cost being used by the GA. One possibility is to lower the maximum test cost $T$ to a value $T^{\prime}$ : 
for instance $T^{\prime}$ can be set to the average value of test costs at which the SPEA2 archive solutions reach $100 \%$ cumulative coverage rate. We decided to implement this second approach in our GA. Since $f_{1}(i): \operatorname{SumCumulCov}(i)$ and $f_{2}(i): \operatorname{Cumul\operatorname {Cov}Cost}(i)$ use $T$ to calculate the objective values, once the maximum test cost $T$ is lowered to $T^{\prime}$, these objective functions will replace $T$ with $T$ '.

More generally, we believe that it is not necessary to wait for all the solutions in the archive population to reach $100 \%$ cumulative coverage rate within $T$ to lower $T$. Our GA allows the test engineer to choose a threshold $S$ (e.g., 80\%) such that if $S$ percent of the archive population reach $100 \%$ cumulative coverage rate within $T$ then the lowering heuristic of $T$ discussed above is used. Again, the rationale is to increase competition between individual solutions.

\subsubsection{Optimization Scheme 2-Specifying a Cumulative Coverage Rate}

A test engineer specifies an expected cumulative coverage rate that is less than $100 \%$, with the default test cost as the total number of transitions. The optimization problem is therefore:

$\min : f_{3}(i): \operatorname{SumDifCov}(i)=\sum_{j=1}^{j=\bar{T}-1}(T-j+1)^{*}(S C-c(i, j))$,

$\min : f_{4}(i):$ CostAtRate $(i)=\bar{T}$, where $\bar{T}=l \mid c(i, l-1)<S C \wedge c(i, l) \geq S C$.

A test engineer may decide to cover a certain amount of data flow information to gain enough confidence in the SUT, while minimizing costs. 


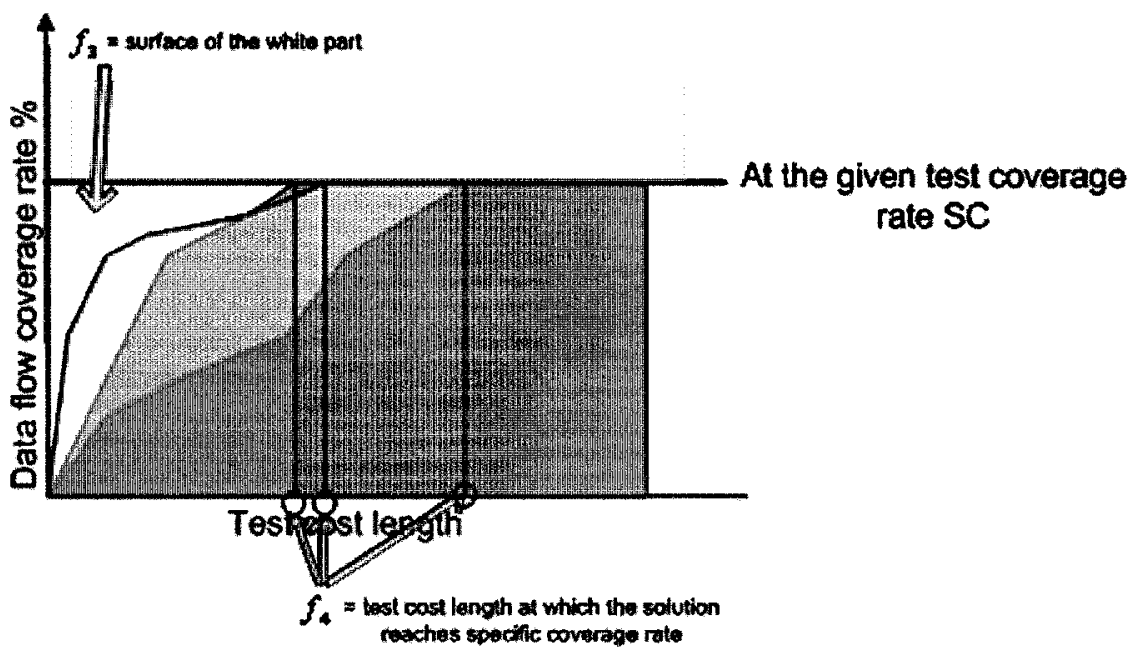

Figure 15: Optimization scheme 2 fitness functions-example

This is illustrated in Figure 15 with three chromosomes (i.e., three coverage rate curves). Objective function $f_{3}(i)$ : SumDifCov(i) is the area bounded by the curve, the straight horizontal line at coverage rate $S C$ and the y-axis. Objective function $f_{4}(i)$ : CostAtRate(i) is the test cost reached at the specific cumulative coverage rate $S C$, indicated by a circle. Using these two objective functions, the chromosomes with the smallest value of the objective $f_{3}(i)$ : SumDifCov(i) and the shortest test cost at the specific coverage rate are kept as non-dominated solutions. There is no need to use the cost reduction heuristic presented in the context of Scheme 1 since no maximum cost is provided by the test engineer and the objective is already to minimize cost.

\subsubsection{Optimization Scheme 3-Specifying 100\% Cumulative Coverage Rate and Maximum Available Test Cost}

The test engineer does not specify any maximum cost or minimum coverage, therefore default values are used: $100 \%$ coverage, and reducing cost. The optimization problem is therefore:

$$
\begin{aligned}
& \max : f_{1}(i): \operatorname{SumCumulCov}(i)=\sum_{j=1}^{j=T}(T-j+1)^{*} c(i, j), \\
& \min : f_{4}(i): \operatorname{CostAtRate}(i)=\bar{T}, \text { where } \bar{T}=l \mid c(i, l-1)<S C \wedge c(i, l) \geq S C .
\end{aligned}
$$




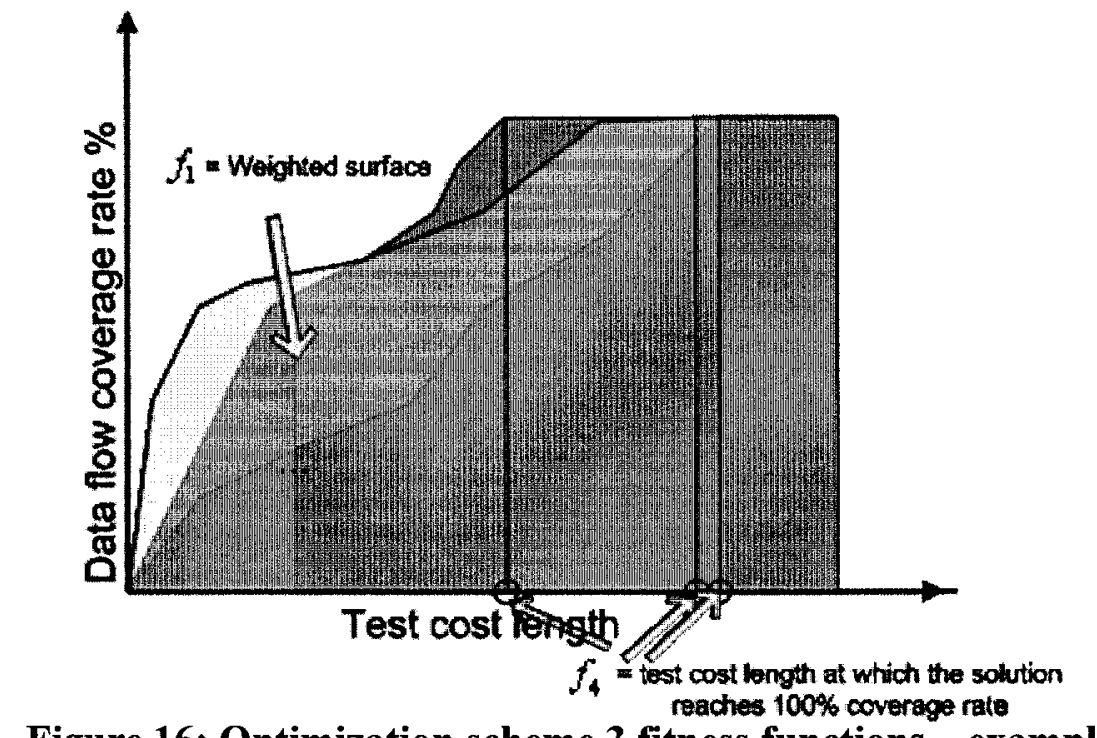

Figure 16: Optimization scheme 3 fitness functions-example

As shown in Figure 16, objective function $f_{1}(i): \operatorname{SumCumulCov}(i)$ is the weighted area bounded by the cumulative coverage rate curve, the $x$-axis (test cost) and the vertical line of the total number of transitions. Objective function $f_{4}(i)$ : CostAtRate(i) is the test cost at which a solution reaches $100 \%$ cumulative coverage rate $(S C=100 \%)$, as indicated by a circle. There is no need to use the cost reduction heuristic presented in the context of Scheme 1 since no maximum cost is provided by the test engineer and the objective is already to minimize cost.

\subsubsection{Optimization Scheme 4-Specifying a Maximum Test Cost and a Minimum Cumulative Coverage Rate}

The test engineer specifies a minimum cumulative coverage rate less than $100 \%$ and a maximum test cost less than the total number of transitions. The optimization problem is therefore:

$$
\begin{aligned}
& \max : f_{1}(i): \operatorname{SumCumulCov}(i)=\sum_{j=1}^{j=T}(T-j+1) * c(i, j) \\
& \max : f_{2}(i): \text { CumulCovCost }(i)=c(i, T) \text {, } \\
& \min : f_{3}(i): \operatorname{SumDifCov}(i)=\sum_{j=1}^{j=\bar{T}-1}(T-j+1)^{*}(S C-c(i, j)) \text {, } \\
& \min : f_{4}(i): \text { CostAtRate }(i)=\bar{T} \text {, where } \bar{T}=l \mid c(i, l-1)<S C \wedge c(i, l) \geq S C \text {. }
\end{aligned}
$$




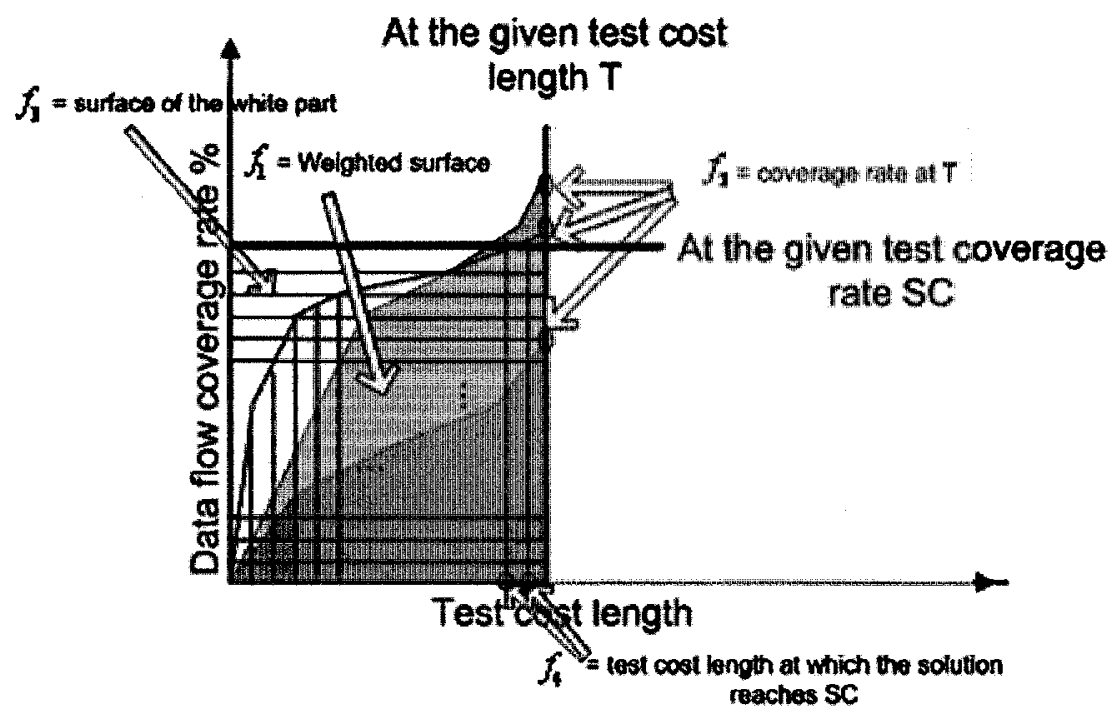

Figure 17: Optimization scheme 4 fitness functions-example

Figure 17 illustrates the four objective functions. From the above objective functions, the chromosome with the best weighted area within test cost $T$ and the chromosome with the best cumulative coverage rate at test cost $T$ will be kept as non-dominated solutions

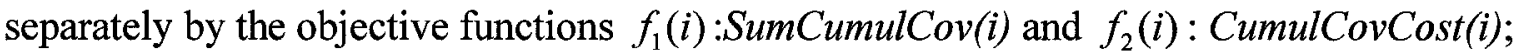
the chromosome with the smallest weighted area bounded by the horizontal line of $S C$, the cumulative coverage rate curve and the y-axis, and the chromosome with the shortest test cost reaching $S C$ will be kept as non-dominated solutions separately by the objective function $f_{3}(i): \operatorname{SumDifCov}(i)$ and $f_{4}(i)$ : CostAtRate(i).

The same heuristic for reducing $T$ as for Optimization Scheme 1 (section 4.3.2) is used in this optimization scheme. Since $f_{1}(i): \operatorname{SumCumulCov}(i), f_{2}(i): \operatorname{CumulCov\operatorname {Cost}(i)}$ and $f_{3}(i): \operatorname{SumDifCov}(i)$ use $T$ to calculate the objective values, once the maximum test cost $T$ is lowered to $T^{\prime}$, these objective functions will replace $T$ with $T^{\prime}$.

Additionally, a similar heuristic is used to reduce $S C$ when the value of $S C$ as specified by the test engineer is so high (and the specified test cost is so small) that no solution can reach $S C$ within $T$ : all solutions will get the same value for objective function $f_{4}(i)$ : CostAtRate(i), and then $f_{4}(i):$ CostAtRate(i) will not contribute to the SPEA2 fitness function any more. Our GA application then automatically lowers the user specified SC 
to $S C$ ' using the following heuristic: $S C^{\prime}$ ' is the average of cumulative coverage rates at $T$ of all solutions in the SPEA2 archive set. Once again, the rationale is to keep the competition between individual solutions by using all the fitness functions. Since $f_{3}(i)$ : SumDifCov(i) and $f_{4}(i)$ : CostAtRate(i) use $S C$ to calculate the objective values, once the minimum data flow cumulative coverage rate $S C$ is lowered to $S C$, these objective functions will replace $S C$ with $S C$ ' to obtain the right values.

One question remains to be answered: When should $S C$ be lowered to $S C$ '? In particular, it is unlikely that any solution in the initial population will reach $S C$ within $T$ since the initial population is constructed randomly, and this does not necessarily mean that $S C$ is too high. We only want to lower $S C$ to $S C$ ' if no solution reaches $S C$ within $T$ after the evolution gets into a stable stage. Different definitions can be given to "stable stage". For example, one might think the evolution gets into a stable stage when the evolution does not improve the population average coverage rate at $T$ by more than $3 \%$ over 50 generations. This is the interpretation of "stable stage" that we used in our GA, and we let the test engineer provide values for the number of generations to monitor for improvements ( 50 in the above example) and the minimum percentage of improvement in population average coverage rate ( 3 in the above example).

\subsection{Genetic Algorithm Operators}

GA operators include crossover (section 4.4.1) and mutation (section 4.4.2). Recall that crossover recombines the heredity material while mutation retains the diversity of the heredity material.

\subsubsection{Crossover: Partially matched crossover}

Our crossover operator is a partially matched crossover (PMX) [6]. Two parent chromosomes are first selected for crossover. Two crossing points are randomly picked for the two parent chromosomes. The section between the two crossover points is called the matching section. The GA then swaps the matching sections in the two parent chromosomes to form two child chromosomes. This will (likely) result into duplicated genes in chromosomes. A matching relationship (noted $\rightarrow$ ) is then identified to remove 
gene duplication, i.e., to identify how the duplicated genes before and after the matching section have to be changed. The matching relation between two genes, one from the child chromosome (gc) in the matching section and one from the parent chromosome (gp), is defined as follows: $g c \rightarrow g p$ if and only if gc and gp appear at the same place in the two chromosomes (i.e., at the same index in the sequences of genes). For each gene $\mathrm{c}$ outside of the matching section in a child chromosome, the GA identifies a gene c' in the matching section of that chromosome that is a duplicate of $c$ (same gene but at different positions in the chromosome). If such a duplicate gene c' exists, the GA recursively applies the matching relationship to c' until the result is a gene that does not appear in the matching section of the chromosome. The result then replaces gene $\mathrm{c}$ in the child chromosome.

Consider the example of Figure 18 with parent chromosomes Parent1 and Parent2 and matching section comprising genes 2 to 5 . Swapping the matching sections results in the "proto" children 1 and 2. In proto child 1, genes A, B and I are duplicated. Gene A at index 0 in the child is a duplicate of gene $A$ at index 5 . There is a matching relationship between $\mathrm{A}$ at index 5 in proto child 1 and $\mathrm{F}$ at index 5 in Parent1, and between $\mathrm{F}$ at index 2 in proto child 1 and $\mathrm{C}$ at index 2 in Parent 1: A $\rightarrow$ F $\rightarrow$ C. Therefore A at index 0 in proto child 1 is replaced by $\mathrm{C}$ to remove duplication. This is repeated for all the genes outside of the matching section.

Parent 1 ABCDEFGH I

Parent 2 EDE |BAGHC

Proto child 1 A B FIBAGH I

Proto child 2 EDEDEFGHC

$$
\begin{array}{lll}
A->F->C, B->E & \text { Child 1 } & \text { CEF I BAGHD } \\
\text { B->E, I->D } & \text { Child 2 } & \text { B I CDEFGHA }
\end{array}
$$

Figure 18: Example crossover

\subsubsection{Mutation (Reordering Mutation): Inversion, Insertion, and Reciprocal}

We used three mutation operators (all these mutation operators only work for the fixed length chromosome):

- Inversion: This operator converts a (randomly selected) sub-sequence of genes into its inverse. For instance, chromosome G1G2G3G4G5G6 would be transformed into 
chromosome G1G2G5G4G3G6, assuming the randomly selected sub-sequence is the one underlined.

- Insertion: This operator randomly takes one gene and randomly moves it at any position in the chromosome. For instance, a chromosome G1G2G3G4G5G6 would be transformed into a chromosome G1G4G2G3G5G6, assuming the gene at position 3 is randomly selected and is randomly moved to position 1 .

- Reciprocal exchange: This operator randomly selects two genes and exchanges their positions. This mutation operator is also called swap mutation in some genetic algorithms frameworks [41]. For instance, chromosome G1 1 2G3G4G5G6 would be transformed into chromosome $\mathrm{G} 1 \underline{\mathrm{G}} 4 \mathrm{G} 3 \underline{\mathrm{G}} 2 \mathrm{G} 5 \mathrm{G} 6$, assuming genes at positions 1 and 3 are randomly selected.

\subsection{Genetic Algorithm Parameters}

The GA efficiency is highly dependent on a number of genetic parameters, such as the population size, the archive size, the number of generation (as a stopping criterion), the crossover rate and the mutation rate. A reasonable combination of genetic parameters could improve the effectiveness of the random search. On the contrary, a poor combination of genetic parameters may lead to premature convergence or loss of good solutions. These parameters should be carefully selected.

The size of the population affects a genetic algorithm's effeciency and effectiveness. A GA with a too small population may not adequately explore the search space. A GA with a too large population may take a long time to converge. Following Goldberg's work [6], De Jong's work applied a population size of 50 in his study for five optimizations. Grefenstette [42] instead suggeseted a population size of 30. In other genetic algorithm applications, a population size of 50 to 100 is widely used by many researchers (e.g., [16, 43]). But for a multi-objective GA, some authors tend to use a larger population size than for a traditional GA, and suggest a size of 30 to 80 for each objective (e.g., [44]). In our GA application, we use a population size of 50 for each objective. 
In SPEA2, the size of the archive set determines how elitist the algorithm is. A small archive set will strengthen the elitism, but the risk is that some (interesting) nondominated solutions may not have room in the archive and may therefore not be kept by the GA. According to [44], archive sizes in the range [1/4, 4] of the population size are acceptable and perform well. But this range was observed to be too wide in our case studies. To keep computation time within reasonable bounds, we set the archive size to half the population size.

The number of generations is also important for an evolution. If the number of generations is too small, the evolution may stop although the solutions may have a high probability of improving. If the number of generations is too large, there may be quite a high time cost. Considering the balance of budget and evolution, as studied in the literature where SPEA2 is used $[12,45]$, we used 500 generations for each trial execution of our GA.

The crossover rate is an important factor to the genetic alogorithm. A moderate crossover rate is able to achieve a good balance between exploration in the whole search space and exploitation to a specific area in the search space1. ${ }^{5}$. According to Goldberg [6], De Jong suggested a crossover rate of 0.6 . Haupt [46] summarized the studies of crossover rate by Grefenstette in 1986 and Schaffer in 1989. Based on their work, a crossover rate in the range $[0.45,0.95]$ is observed to perform well. Consistent with their findings, we set the crossover rate to 0.7 .

The mutation rate also affects the performance of the genetic algorithm. Following Goldberg's work [6], De Jong studied mutation rates from 0.001 to 0.1. Based on De Jong's analysis, the GA with mutation rate 0.1 is close to a random search. The mutation rates of $0.001,0.005,0.01,0.02$ have similar performances, though the mutation rate 0.001 is a little bit better than the others (this value is applied in De Jong's subsequent studies). Later, Grefenstette [42] suggests a mutation rate 0.01 based on his work. In [44],

\footnotetext{
${ }^{5}$ Exploration refers to how widely the algorithm searches/explores the whole search space whereas exploitation refers to how deep the algorithm searches into an optimal area to find the best solution. An hill climbing algorithm is a strategy to do exploitation without considering exploration, and a random search algorithm is a strategy to do exploration without considering exploitation.
} 
the authors report that based on their study on 250,500 , and 750 items knapsack problems, a relatively high mutation rate should be used to achieve the best performance. They suggest a mutation rate of 5 divided by the length of chromosomes. Base on their size of the problem, the mutation rate is respectively $0.02,0.01$ and 0.007 which are in the range of De Jong's study. But this was not suitable for two of our case studies due to their small sizes (20 and 165 genes). Therefore we selected the widely used GA mutation rate of $0.01[12,17,42]$. Note that we will study the impact of different mutation rate values for our largest problem.

Based on the above discussion, we summarize the GA parameters in Table 2.

\begin{tabular}{|l|l|l|}
\hline & $\begin{array}{l}\text { Optimization } \\
\text { Scheme 1,2,3 }\end{array}$ & $\begin{array}{l}\text { Optimization } \\
\text { Scheme 4 }\end{array}$ \\
\hline Generations & 500 & 500 \\
\hline Population Size & 100 & 200 \\
\hline Archive Set Size & 50 & 100 \\
\hline Crossover Rate & 0.7 & 0.7 \\
\hline Mutation Rate & 0.01 & 0.01 \\
\hline
\end{tabular}

Table 2: GA parameters 


\section{DESIGN AND IMPLEMENTATION}

In this chapter, we present how we designed and implemented our multi-objective GA application in section 5.1 and section 5.2. Also, we discuss the issues that we encountered in the initial experiment, and provide our analysis and solution in section 5.3.

\subsection{Design}

In our problem, a test case sequence is an ordered set of test cases from a test suite. A test case sequence is modeled as a chromosome where genes are test cases. Each test case contains one or several ordered transitions. Transitions are considered equivalent costwise, e.g., they are assumed to take the same time to execute. Thus the test cost of a test case is equal to the number of transitions it contains. Each transition may cover zero or several definitions or definition-use (DU) pairs: a transition covers a definition when it contains a definition of a variable; a transition covers a DU pair when it contains a use of a variable, there exist a transition earlier in the test case containing a definition of that variable, and none of the transitions in between (re-)defines that variable. The conceptual model of the concepts we have to model and their relationship is shown in Figure 19.

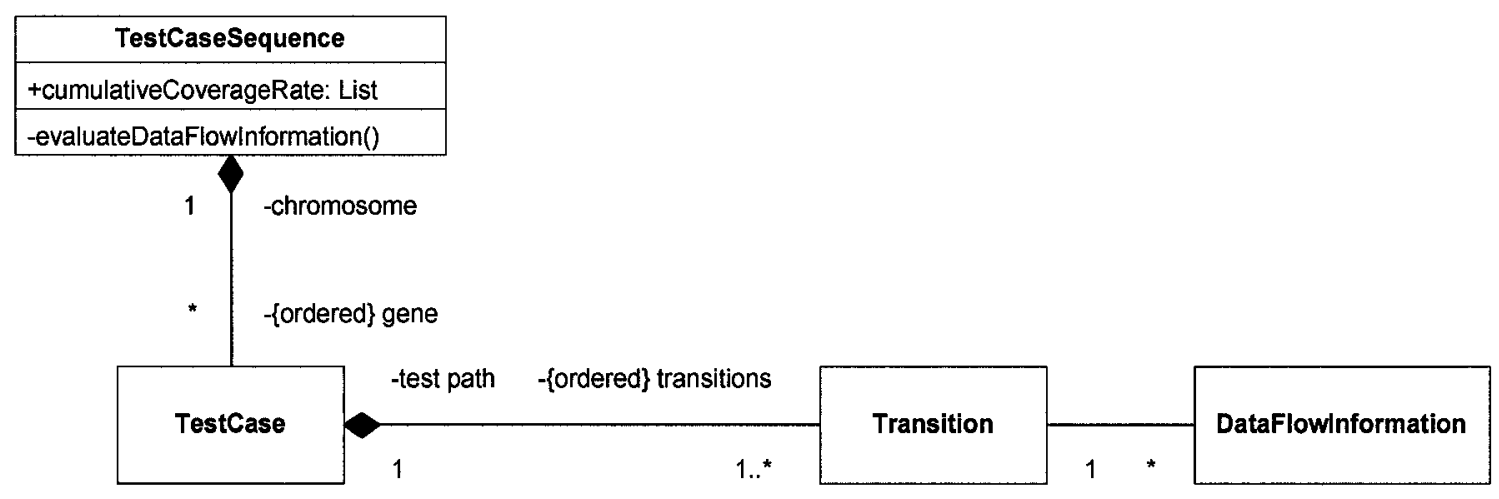

Figure 19: Model of test case sequence and data flow information

Method evaluateDataFlowInformation() in Figure 20 evaluates the list of cumulative coverage rates of a chromosome. The inputs to this method are the user specified minimum cumulative coverage $S C$ and maximum cost $T$, as well as the total number of transitions and the total number of definitions or the total number of DU pairs. The 
specified $S C$ and $T$ are the stop criteria for the calculation of data flow in a chromosome. Method docalculate() calculates the data flow increments by comparing the data flow information a transition covers with the data flow information its predecessors (in the chromosome) cover, and eventually gets the cumulative coverage rate for the current test cost (variable $\mathrm{CCR}$ ). Each element of cumulative coverage rates is a vector that contains a cumulative coverage rate and a test cost.

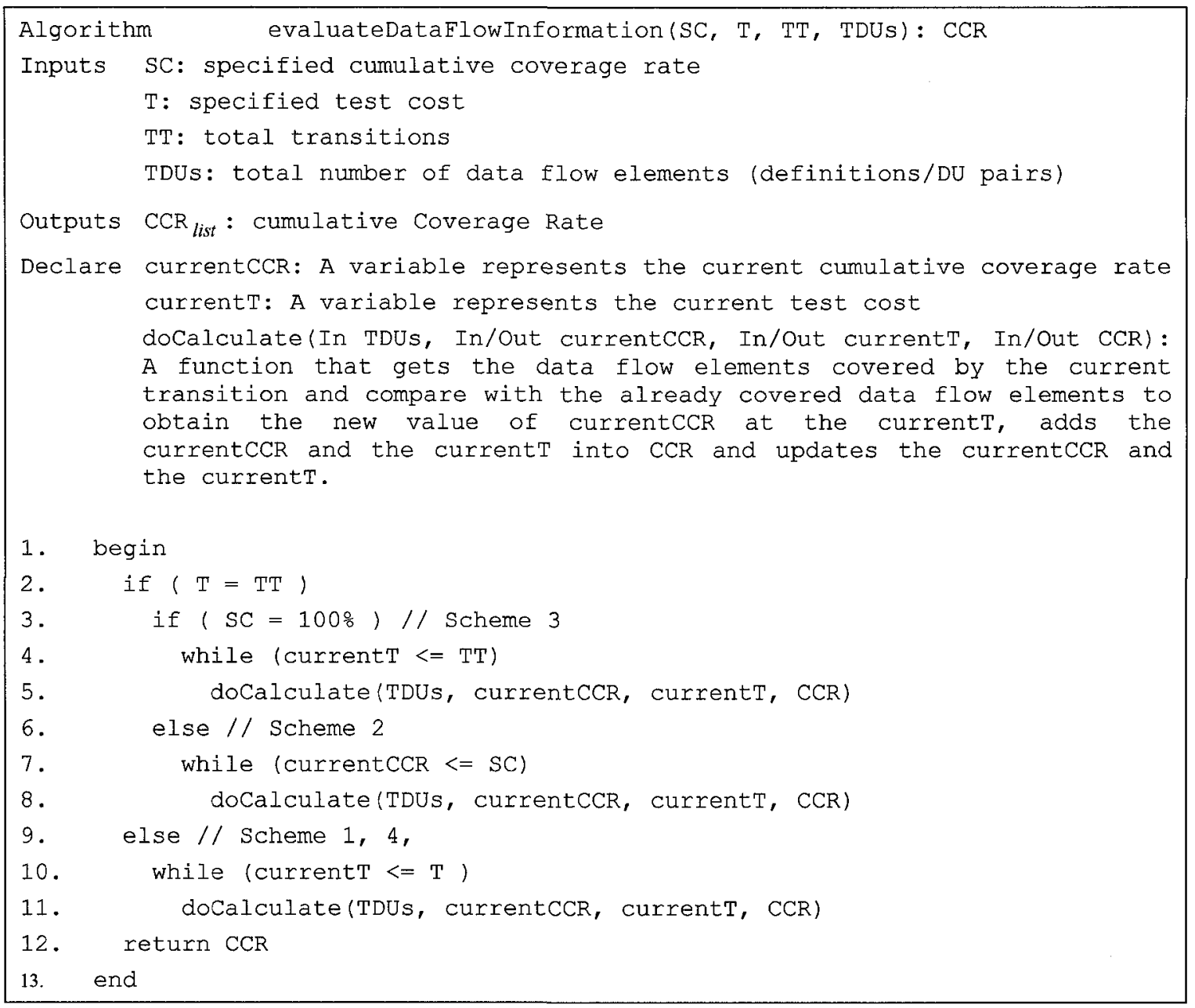

Figure 20: Algorithm for evaluating coverage of data flow information

In our design, two parameters, the user specified cumulative coverage rate (SC) and the user specified test cost (T), define the optimization scheme. This is illustrated by the different conditions in the algorithm of Figure 20: Scheme 3 appears on lines 4-5, Scheme 2 appears on lines 7-8, and Schemes 1 and 4 appear on lines 10-11. Other inputs are required to evaluate a chromosome's fitness such as the total number of data flow elements and the total number of transitions. Appendix B provides a method for obtaining 
data flow elements (definitions/DU pairs) of operation contracts covered by each transition in each test case. All the above information is provided by the user through an input file. All the GA parameters (recall section 4.5) are also provided by that input file. An example of an input file is provided in Appendix C.

In the output of the GA, all test case sequences that satisfy a test engineer's requirements are presented with each objective value and the list of cumulative coverage rates. An example of an output file is provided in Appendix D.

\subsection{Implementation}

The implementation is based on the open source genetic algorithm framework JGAP [41]. JGAP is a Java-based Genetic Algorithm and Genetic Programming framework. It provides basic evolutionary mechanisms that allow users to easily use their own genetic operators. Generally, when a user wants to apply JGAP to solve his/her own problem, he/she first needs to decide the form in which a candidate solution will be represented. This framework defines two interfaces for that purpose: Ichromosome and Gene. We implemented a chromosome class TestCaseSequence and a gene class Transition.

Each chromosome contains a sequence of genes. It is decoded before the fitness function is called to assign a fitness value. The decode procedure is accomplished by method evaluateDataflowInformation(). After that, the list of cumulative coverage rates is obtained. The objective functions are then called to assign objective values based on the cumulative coverage rates. These objective function values are used by the SPEA 2 fitness function to obtain a value of strength as the chromosome's fitness value.

Based on an available SPEA2 implementation on the JGAP framework [47, 48], we implemented the SPEA2 fitness function, the objective functions, the crossover operators and the mutation operators. The class diagram based on JGAP is presented in Figure 21. The classes and interfaces from the SPEA2 implementation on the JGAP framework [47] are marked in white; our implemented classes are marked in light grey. We implemented our own objective functions (section 4.3.1), the genetic operators (e.g. the crossover operator PartiallyMatchedXover and the mutation operators InsertMutation and 
InverseMutation), the chromosome TestCaseSequence, the gene TestCase and the entity Transition which is used to build the data relationship between InputProperties and Testcase.

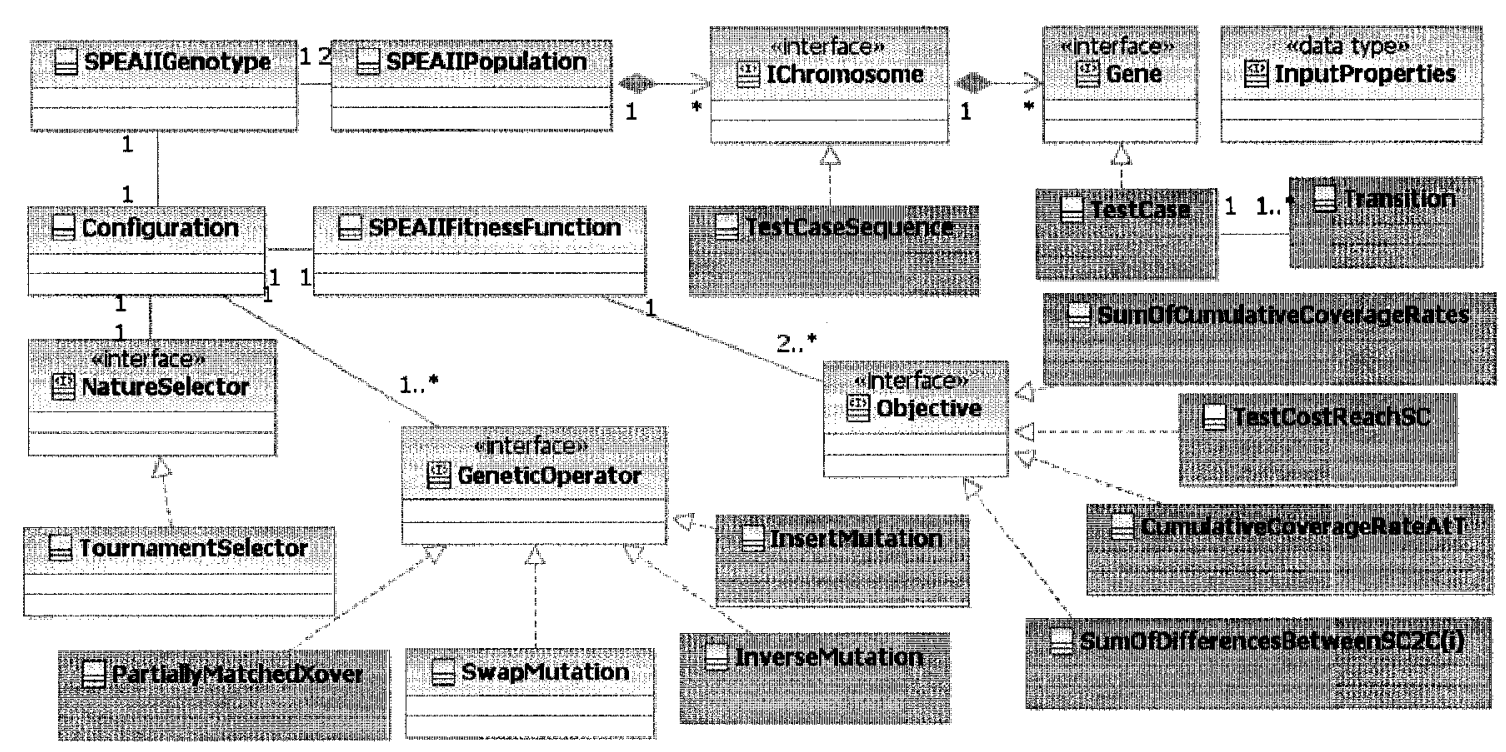

Figure 21: Class diagram of the implementation based on JGAP framework

\subsection{Issues in our GA Evolution}

During our initial uses of our multi-objective GA, we identified two issues which we discuss here.

\subsubsection{Effective Genes}

In our implementation, the raw data of the data flow coverage rate is used to calculate the objective values. When the data flow coverage rate reaches $100 \%$ or a user-specified coverage rate at the $i$-th gene (test case), any change to the chromosome (in particular crossover operations) from the $i+1$-th gene to the end of the chromosome will not change any objective value, then will not change the fitness value, since changes are made to parts of the chromosome that are not used for objective evaluation purposes. This is illustrated by the short example in Table 3, where a test suite of ten test cases (rows) covers 20 data flow pieces (columns) with the simplifying assumption that each test case contains one transition. 


\begin{tabular}{|l|c|c|c|c|c|c|c|c|c|c|c|c|c|c|c|c|c|c|c|c|}
\hline TCIDataFlow & A & B & C & D & E & F & G & H & I & G & K & L & M & N & O & P & Q & R & S & T \\
\hline TC1 & & X & & X & & X & & X & & & & X & & & & X & & & X & \\
\hline TC2 & $\mathrm{X}$ & & & $\mathrm{X}$ & & & $\mathrm{X}$ & & & & & & & $\mathrm{X}$ & & & $\mathrm{X}$ & $\mathrm{X}$ & & \\
\hline TC3 & & & $\mathrm{X}$ & & & $\mathrm{X}$ & & & & $\mathrm{X}$ & & & $\mathrm{X}$ & & & $\mathrm{X}$ & $\mathrm{X}$ & & $\mathrm{X}$ & \\
\hline TC4 & & & & & $\mathrm{X}$ & & & & $\mathrm{X}$ & & & $\mathrm{X}$ & & $\mathrm{X}$ & & & & & & $\mathrm{X}$ \\
\hline TC5 & & $\mathrm{X}$ & & & & & $\mathrm{X}$ & & & & $\mathrm{X}$ & & & & $\mathrm{X}$ & & & & & \\
\hline TC6 & $\mathrm{X}$ & & & & & & & & $\mathrm{X}$ & & & & $\mathrm{X}$ & & & & & & & \\
\hline TC7 & & $\mathrm{X}$ & $\mathrm{X}$ & & & & & & & & $\mathrm{X}$ & & & & & $\mathrm{X}$ & & & $\mathrm{X}$ & $\mathrm{X}$ \\
\hline TC8 & & & & $\mathrm{X}$ & & & & $\mathrm{X}$ & & & $\mathrm{X}$ & & & & $\mathrm{X}$ & & & & & \\
\hline TC9 & & $\mathrm{X}$ & & & & & & & & $\mathrm{X}$ & & & & & & & $\mathrm{X}$ & $\mathrm{X}$ & & \\
\hline TC10 & & & & & $\mathrm{X}$ & & & & & & & & & $\mathrm{X}$ & & $\mathrm{X}$ & $\mathrm{X}$ & & & \\
\hline
\end{tabular}

Table 3: A short example: a test suite with 10 test cases covers 20 data flow pieces

Further assume that the two parents below are used in a crossover operation (partially matched crossover described in Section 4.4.1), with two randomly selected crossover points of 6 and 8 (as indicated in Figure 22). Since the coverage rate of the two parents has already reached $100 \%$ by gene 6 (first crossover point), crossover changes after 6 do not change the data flow coverage rate: the two generated children do not improve the population; they are actually identical to the two parents from the point of view of the fitness function.

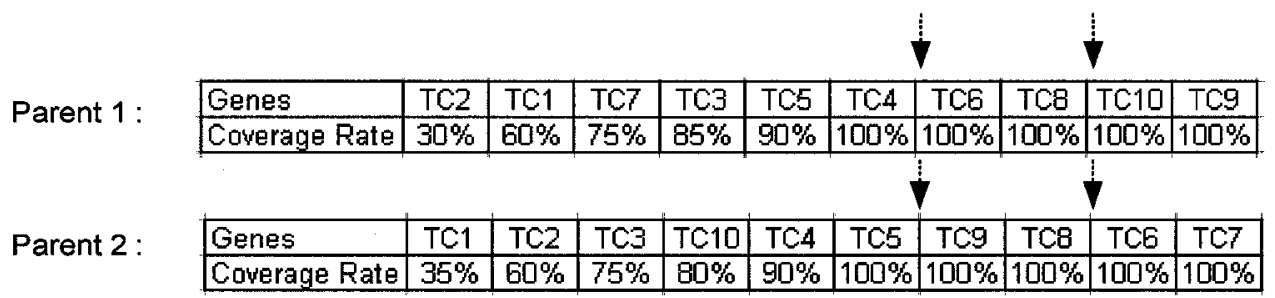

After Partially Matched Crossover

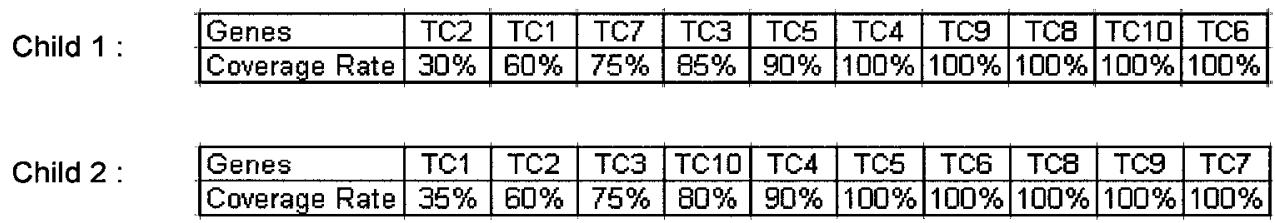

Figure 22: Partially matched crossover operator

In some extreme cases, if it takes only the first $20 \%$ of the genes of a chromosome to reach a $100 \%$ data flow coverage rate or the user-specified coverage rate, all genetic 
operations that take place in the following $80 \%$ of the genes do not help genetic evolution. If the two crossover points are randomly selected, there is an $80 \%$ probability that the genetic operations will not help genetic evolution. This in essence reduces the crossover rate: the smaller the number of genes actually used in fitness evaluations, the larger the reduction in crossover rate.

To avoid such a situation, the crossover points need to be selected carefully and we used the following heuristic when applying the partially mapped crossover operator.

First, we ascertain the gene at which the data flow coverage rate reaches $100 \%$ or the user-specified coverage rate SC. This gene and its preceding genes are called the effective genes since they are important for fitness evaluation. We then make sure the first crossover point is always located within the effective genes. This ensures that some effective genes get modified in the child. We also make sure the second crossover point is always located outside the effective genes. This ensures that genetic material other than in the effective genes will always appear in children. Reusing the above example, the first crossover point will always be located within the first six genes (say 2), and the second crossover point will be always located after the seventh gene (say 7), resulting for instance in the two children of Figure 23.

\begin{tabular}{|c|c|c|c|c|c|c|c|c|c|c|}
\hline \multirow{3}{*}{ Parent 1: } & \multicolumn{5}{|c|}{$\nabla$} & \multicolumn{5}{|c|}{7} \\
\hline & Genes & TC2 & TC1 & TC7 & TC3 & TC4 & TC6 & $T C 8$ & TC10 & TC9 \\
\hline & Coverage Rate & $30 \%$ & $60 \%$ & $75 \%$ & $85 \%$ & \begin{tabular}{|l|l|}
$90 \%$ & $100 \%$ \\
\end{tabular} & $100 \%$ & $100 \%$ & $100 \%$ & $100 \%$ \\
\hline \multirow{3}{*}{ Parent 2: } & \multicolumn{5}{|c|}{ i } & \multicolumn{5}{|c|}{7} \\
\hline & Genes & TC1 & TC2 & TC3 & TC10 & TO5 & TC9 & $\mathrm{TCB}$ & TC6 & TC7 \\
\hline & Cowerage Rate & $35 \%$ & $60 \%$ & $75 \%$ & $60 \%$ & $100 \%$ & $100 \%$ & $100 \%$ & $100 \%$ & $100 \%$ \\
\hline
\end{tabular}

After Partially Matched Crossover

\begin{tabular}{|c|c|c|c|c|c|c|c|c|c|c|c|}
\hline \multirow{2}{*}{ Child 1: } & Genes & TC2 & TC1 & TC3 & TC10 & TC4 & TC5 & TC9 & TC8 & TC7 & TCE \\
\hline & Coverage Rate & $30 \%$ & $60 \%$ & $75 \%$ & $80 \%$ & $90 \%$ & $100 \%$ & $100 \%$ & $100 \%$ & $100 \%$ & $100 \%$ \\
\hline \multirow[t]{2}{*}{ Child 2} & \begin{tabular}{|l} 
Genes \\
\end{tabular} & TC1 & TC2 & TC7 & $T 03$ & TC5 & TC4 & TC6 & TC8 & $\mathrm{TC9}$ & TC \\
\hline & Coverage Rate & $35 \%$ & $60 \%$ & $75 \%$ & $85 \%$ & $90 \%$ & $100 \%$ & $100 \%$ & $100 \%$ & $100 \%$ & $100 \%$ \\
\hline
\end{tabular}

Figure 23: Partially matched crossover with the effective genes 
It is very possible that two parents have different numbers of effective genes. In that situation, the crossover points will be selected according to the parent chromosome that has the smaller number of effective genes.

Effective genes do not have an impact on the mutation rate as they do on the crossover rate. When applying a crossover, the crossover rate applies to the chromosome, i.e., the genetic algorithm first decides if two chromosomes will be crossed over (according to the crossover rate), and then randomly select two crossover points to do the crossover. Crossover points located after the effective genes will produce chromosomes identical in phenotype to the parents, thereby reducing the actual crossover rate. On the other hand, when applying mutation, the mutation rate applies to each gene in the chromosome, i.e., the genetic algorithm goes through each gene in a chromosome and uses the same probability (the mutation rate) to decide whether to mutate the gene or not. Mutations happening to the effective genes or non-effective genes have therefore the same probability of being mutated: effective genes cannot reduce to the mutation rate. It is also worth noting that some mutations (in particular the swaping) may bring genes from the non-effective section to the effective section.

\subsubsection{Genotype and Phenotype}

Some chromosomes may have different sequences of genes, but may have the same objective values, while others may have different objective values but almost identical sequences of genes. In other words, some chromosomes may have different genotypes but identical phenotype, while others may have different phenotype but almost identical genotypes. Both the genotype and the phenotype are important characteristics of chromosomes for evolution purposes. The phenotype (objective values) tell us whether chromosomes are good solutions for the objective(s) whereas the genotype may indicate promising genetic material for future evolution though the chromosomes with this promising genetic material may not currently be good solutions.

In some multiple-objective genetic algorithms, such as SPEA2, when the number of nondominated solutions exceeds the size of the archive set, a truncation mechanism is employed to obtain the right number of chromosomes in the archive set: typically, some 
of the non-dominated solutions, i.e., some of the good solutions phenotype-wise, are removed from the archive set. For instance, if two solutions have the same objective values, one of them will be removed since they are thought to be redundant solutions. If two solutions have similar objective values, one of them may be removed to maintain diversity in phenotype. In SPEA2, when the number of non-dominated solutions exceeds the size of the archive set, the truncation operator removes the solutions that are close to others, in terms of phenotype: SPEA2 uses a neighbor distance that is based on the difference between objective values. The genotype is therefore not used.

Using only the phenotype when comparing non-dominated solutions may be a problem. Indeed, two chromosomes with identical phenotype may have very different genotype and the two genotypes may be equally promising for future offspring. If only the phenotype is used when truncating, there is a risk that only chromosomes with similar genotypes be kept, thereby leading to low diversity in terms of genotype. In our studies, we did find that our final GA solutions (i..e, test case sequences) bear some similarity in terms of genotype, which means many solutions look like each other with only a very small difference in the sequences of genes. It is however not clear whether this is a result of the specifics of the case studies or the result of SPEA2 (and we may have lost good solutions). However, all currently available multi-objective genetic algorithms are working on the phenotype rather than on the genotype. We believe that both phenotype and genotype should be considered, and we will look into this during future work. 


\section{CASE STUDIES}

In this chapter we will look at three case studies on three representative systems that exhibit state-based behaviors: two purely event-based real-time systems (a cruise control system and a VCR system) and a data structure class. Based on the three systems, we will develop a test suite and apply the GA application to order test cases. Then we will compare the GA results, using the different schemes, with randomly ordered test case sequences to see how well the GA ordered test case sequences increase their data flow coverage rates and whether the GA ordered test case sequences could help users to detect faults earlier. We first discuss the design of the case studies in section 6.1. We then look at the results for the three case study systems in sequence (sections 6.2 to 6.4). Last we conclude by summarizing the results in section 6.5 .

\subsection{Case Studies Design}

In this section, we discuss the design of the three case studies. First of all, we have to decide of a test case generation strategy. In the light of the discussion of section 3.1.2, we decided to use Briand et al.'s algorithm to generate test cases from a transition tree. Test cases therefore all start from the initial state of the state machine: recall the discussion in section 4.1.2. Note however that the whole strategy can be applied to any test suite made of transition sequence, regardless of the criterion being used to generate them.

We selected two data flow coverage criteria, namely all-definitions and all-DU pairs (recall section 4.1.4.2). The all-definitions criterion requires that for each definition of a variable, a test suite should cover a definition clear path from this definition to at least one use. The all-DU pairs criterion requires that for each definition of a variable, a test suite should cover a definition clear path from this definition to each use.

In order to compare the GA ordered test case sequences, using different schemes, and randomly ordered test case sequences (section 6.1.1), we define two comparison criteria (section 6.1.2). One of the comparison criteria requires the presence of faults and we 
discuss the generation of faults (a.k.a. mutants ${ }^{6}$ ) in section 6.1.3. We then briefly present the three cases studies in section 6.1.4.

We proceeded as follows during the three case studies. For each of the three systems, we first generated a test suite based on Briand et al.'s algorithm, which covers more data flow information than Binder's algorithm (section 3.1.3). We then applied the multiobjective GA, using the four different schemes separately, to generate test case sequences. Note that some of the schemes require that the test engineer provide inputs (such as a maximum test cost). We tried different values for these inputs to study the impact of those values on trends in coverage and mutation scores. Then we seeded mutants into the source code of the case study system and executed GA-evolved test case sequences to obtain their mutant detection capability (one of the two comparison criteria, discussed in section 6.1.2). In addition, for comparison purposes, we generated random orders of test case sequences (section 6.1.1) and determined their mutant detection capability. Finally, we compared the data flow coverage rate and the mutant detection capability (section 6.1.2) of the different orders to verify whether the GA-evolved test case sequences detect these mutants earlier, and whether with a user-specified test cost, the GA-evolved test case sequences detect more mutants.

During our case studies, we want to answer three research questions:

Q1: Can the data flow information derived at the model level help testers to identify interesting (in terms of data flow coverage, in term of fault detection) execution priorities of test cases?

Q2: Do the GA-evolved test case sequences detect defects in source code earlier than randomly ordered test case sequences, or detect more defects within a limited budget?

\footnotetext{
${ }^{6}$ In the literature on mutation testing and the use of mutants to compare testing techniques, modifications of the source code are referred to as mutants and are created from mutation operators. And one refers to the number of mutant discovered (or killed) by a test suite as the mutation score. To avoid confusion with the notion of mutation in the context of a Genetic Algorithm, we use the word mutant to refer to source code modifications. We therefore use terms like mutant score, mutant operators, instead of mutation score and mutation operator.
} 
Q3: What is the variation (standard deviation) in defect detection of GA-evolved test cases sequences (using data flow information at the model level), and how does it compare with the variation in defect detection of random test case sequences?

\subsubsection{Random Test Case Sequences}

Random test case sequences are generated for comparing data flow coverage rates and mutant detection rates with GA ordered non-dominated test case sequences. After one genetic execution, we will get a SPEA2 archive set. From this set, we could get at least one to at most all of the archive set of non-dominated solutions. To perform a fare comparison we will therefore generate the same number of random test case sequences as the size of the SPEA2 archive set at the end of the evolution.

Since we have one genetic execution for each optimization scheme, we will generate a set of random test case sequences for each scheme. Based on different optimization schemes, we will use different methods to generate random test case sequences. For schemes 1, 3 and 4, since all GA ordered non-dominated test case sequences have the same test costs, we simply generate a set of random test case sequences with the same test costs as the GA ordered sequences. For scheme 2, since all GA ordered non-dominated test case sequences might have different test costs (at which they reach $S C$ ), we generate a set of random test case sequences and stop their construction when they reach $S C$.

When the non-dominated test case sequences presented by our GA application have the same test costs (schemes 1, 3, and 4), we compare them with random test case sequences cost by cost from test cost 1 to the end of these sequences (the common cost value). When the non-dominated test case sequences presented by our GA application have different test costs (scheme 2), we compare them with random test case sequences cost by cost from test cost 1 to the maximum cost of the non-dominated test case sequences. (This is discussed in more details in the following section.) We will discuss the criteria to compare non-dominated test case sequences and random test case sequences in the next section. 


\subsubsection{Criteria to Compare Test Case Sequences}

In our case studies, two important properties will be compared: the data flow coverage rate (section 6.1.2.1) and mutant detection rate (section 6.1.2.2). Because at the end of each genetic algorithm execution our multi-objective GA presents us with a set of candidate test case sequences, we will compare the average rate of the whole set with the average rate of a set of randomly ordered test case sequences. We will also look at distributions of those rates. Section 6.1.2.3 discusses our selection of input values (minimum cumulative coverage and/or maximum cost) to simulate what a test engineer might want.

\subsubsection{Average Data Flow Cumulative Coverage Rate}

In section 4.1.4.2, we defined the cumulative coverage rate $c(i) . c(i)$ equals the result of the number of definitions / DU pairs covered up to the $i$-th test cost divided by the number of all known definitions / DU pairs. We also defined $c(k, i)$ as the data flow coverage rate at test cost $i$ for the $k$-th test case sequence in section 4.3.1. With $N$ test case sequences, the data flow coverage rate at each test cost $i$ on average is therefore calculated as: $D F(i)=\frac{1}{N} \sum_{k=1}^{N} c(k, i)$.

In our case study, we compare the series of data flow coverage rates $D F(i)$ from the GAevolved test case sequences with the series of data flow coverage rates $D F(i)$ from a set of random test case sequences.

Recalling optimization scheme 2, all non-dominated chromosomes in the SPEA2 archive set might take different test costs to reach a user specified cumulative coverage rate $S C$. For example, solution 1 reaches $S C$ at test cost $l$, solution 2 reaches $S C$ at test cost $m$ and $m$ is greater than $l$. Since solution 1 does not contain any data flow cumulative coverage rate value at test costs $[l+1, m]$, we cannot use the above formula to calculate the average data flow cumulative coverage rate for test costs $[l+l, m]$. We have two options: 1 . identify the solution whose data flow cumulative coverage rate reaches $S C$ with the shortest test cost $T_{\text {shortest }}$ in the SPEA2 archive set and stop calculating the average data 
flow cumulative coverage rates at $T_{\text {shortest }}$ for all the sequences; 2 . calculate the average data flow cumulative coverage rate with only chromosomes who have non-zero cumulative coverage rates at each test cost value.

For option 1, the average data flow cumulative coverage rate at each test cost on average is re-defined as:

$$
D F(i)=\frac{1}{N} \sum_{k=1}^{N} c(k, i), i \leq T_{\text {shorrest }}
$$

For option 2, the average data flow cumulative coverage rate at each test cost on average is re-defined as:

$$
D F(i)=\frac{1}{\left|N^{\prime}\right|} \sum_{k \in N^{\prime}} c(k, i), N^{\prime}=\{k \mid c(k, i)>0 \wedge c(k, i) \leq S C\}
$$

Option 1 allows us to easily get the average data flow cumulative coverage rate, but it does not show the data flow cumulative coverage rate after the shortest test cost $T_{\text {shortest }}$. Option 2 leads to slightly more difficult calculations, but it shows all information about non-dominated solutions. Plus, for all cost values between cost 0 and $T_{\text {shorest }}$ the two options provide identical results. However, one side effect of option 2 is that the $D F(i)$ function may no longer be monotonic (i.e., it increases) for cost values greater than $T_{\text {shortest }}$ since the number of solutions considered in the average may decrease as cost increases. For example, consider two solutions with data flow coverage rates $\{0.1,0.2$, $0.3,0.4,0.5,0.6,0.7,0.8\}$ and $\{0.3,0.6,0.7,0.8\}$ reaching 0.8 at test costs 8 and 4 , respectively. The shortest test cost $T_{\text {shorest }}$ equals 4 (for the second solution). The average data flow coverage rate at each cost is $\{0.2,0.4,0.5,0.6,0.5,0.6,0.7,0.8\}$, which increase for costs values smaller than $T_{\text {shortest }}$ but is no longer monotonic (it increases and decreases) for cost values greater than $T_{\text {shortest }}$. In the end, we select option 2 as it accounts for all the information in chromsomes. We will however keep in mind the counter-intuitive (for a cumulative metrics) situation discussed above when analyzing results. 
When we present the comparison results of GA-evolved sequences and random sequences, we also want to show the variance between the sequences. We foresee that random sequences may have large variances. Therefore, when we present the comparison result of GA-evolved sequences and random test case sequences, we do not only present the average data flow coverage rate at each test cost, but also we present their mean, minimum and maximum values at test cost $i$.

\subsubsection{Average Mutant Detection Rate}

Authors have defined a metric to compare orders of test cases in terms of fault detection: the average of the percentage of faults detected (APFD) metric [38], [39], and its extension APFDc ("cost-cognizant APFD") that accounts for varying test case costs and varying faults severities [49]. Though it has been defined in the context of regression testing, specifically for comparing prioritizations of regression tests, this metric may apply to our context. To compare two orders of test cases using APFD, the two test case orders need to have the same number of test cases. This is however not necessarily the case of test case sequences returned by our multi-objective GA. Consider for example scheme 2 , whereby the test engineer specifies a minimum cumulative coverage rate to be achieved. Different test case sequences with varying numbers of test cases may be returned by the GA. Even with scheme 1, whereby the test engineer specifies a maximum cost, test case sequences with various lengths may be returned by the GA, for instance if it is feasible to achieve $100 \%$ cumulative coverage with the specified cost limit.

We therefore defined our own metric to compare the fault detection effectiveness of different test case sequences. The mutant rate of one test case sequence at test cost $i$ is the number of detected mutants up to the $i$-th test cost divided by the number of all known detectable mutants, written as:

$m(i)=\frac{\text { TheNumberOfDetectedMutationUntil }(i)}{\text { TheTotalNumberOfDetectableMutations }}$

With $N$ test case sequences, the mutant rate at each test cost on average is calculated as: 
$M(i)=\frac{1}{N} \sum_{k=1}^{N} m(k, i)$ where $m(k, i)$ is the mutant rate at test cost $i$ for the $k$-th test case sequence.

In our case study, we compare the series of mutant rate $M(i)$ from the GA-evolved test case sequences with the series of mutant rate $M(i)$ from a set of random test case sequences. As well, in the case study results, we also present mean, minimum and maximum mutant rate values at each test cost $i$.

For optimization scheme 2, we apply the same method to calculate the average mutant detection rate as the one we discussed to calculate the average data flow cumulative coverage rate (section 6.1.2.1): i.e., we continue past the minimum cost but perhaps with varying (actually decreasing) numbers of sequences. Remember from section 6.1.1 that in scheme 2, since all GA ordered non-dominated test case sequences might have different test costs (at which they reach $S C$ ), we generate a set of random test case sequences and stop their construction when they reach $S C$. So these random sequences may too have different lengths, i.e., costs.

\subsubsection{Selection of user-defined inputs for comparison purposes}

In our GA application, a tester has to define his/her expected data flow coverage rate and the available budget which is expressed as a maximum test cost. In our case studies, we therefore need to select values for these two user input data for each scheme requiring user input. Often, achieving $100 \%$ coverage turns out to be too expensive. Testers often do not expect $100 \%$ coverage but on the other hand they do not want a too low data flow coverage rate since otherwise it is easy to miss a lot of defects. It is well accepted, thanks to empirical results (e.g., $[3,50])$, that a data flow coverage rate around $90 \%$ or above is a reasonable target. We therefore select data flow coverage rates of $90 \%$ and $80 \%$ (both for all-definitions and all-DU pairs) as inputs for optimization scheme 2 and scheme 4 . Usually a tester expects to cover a high data flow coverage rate with a short test cost. Since at the end of genetic evolution, our GA application can provide the user the reduced test cost which satisfies his/her requirement in terms of data flow coverage rate, in scheme 1, we just set a quite large test cost (actually, close to the maximum cost, i.e., 
the full test suite, such as $99 \%$ of the total cost of the full test suite) and wait for the GA to reduce test costs. (Note that setting maximum cost to $100 \%$, instead of $99 \%$, would correspond to scheme 3 rather than scheme 1.) In scheme 4 , where we set a maximum test cost and a minimum cumulative coverage rate, we set the cost to $80 \%$ and $90 \%$ of the total cost of the full test suite because we want to reduce test costs in proportion to the specified data flow coverage rates (which we set to $80 \%$ and $90 \%$ ). To complement the study of the impact of a specified test cost, we then use test cost values a bit larger and smaller than the one discovered by the GA. For example, if with optimization scheme 4 and user input data $T=90$ and $S C=90 \%$ our GA application reduces test cost $T$ to $T^{\prime}=45$ (i.e., we do not need a cost of 90 to reach $S C$, but a cost of 45 is sufficient), we then try optimization scheme 4 again twice, with pairs of input data $(T=50, S C=90 \%)$ and $(T=40$, $S C=90 \%$ ). In the former case, we expect our GA application to return similar results as with input ( $T=90, S C=90 \%$ ). Since these results might be very similar to the initial inputs, we will not report all detailed results. In the latter case, we expect our GA application to present solutions that can reach a cumulative coverage $S C^{\prime}$ (lower than $S C$ ) at the user specified test cost $T=40$ and we will discuss them when presenting results.

As a result, for optimization scheme 1 (where one specifies a maximum test cost), we first set a large maximum test cost such as $99 \%$ (of the total number of test cases in the tets suite) just to ensure that most solutions can reach $100 \%$ data flow cumulative coverage rate. Then we use other values of cost (smaller and larger than what the GA returns). For optimization scheme 2 (where one specifies a minimum cumulative coverage rate), we set the minimum data flow coverage rate to $80 \%$ and $90 \%$. For optimization scheme 3 (where one wants to achieve $100 \%$ cumulative coverage, regardless of cost), we do not need to set any user specified data. For optimization scheme 4 , we first set a large maximum test cost, such as $80 \%$ or $90 \%$ of total test costs, combined with data flow coverage rate $80 \%$ and $90 \%$. Then we use other values of costs (smaller and larger than what the GA returns), as discussd above, with the same data flow coverage rate $80 \%$ and $90 \%$. 


\subsubsection{Mutant Operators}

In our case studies, mutants are automatically generated by a third party tool, Muclipse, which is the Eclipse plugin of MuJava [51]. MuJava is a mutation system for Java programs. MuJava automatically and systematically generates traditional and class-level mutants. When it generates mutants based on mutant operators according to the user's selection of mutant operators, without any bias it seeds as many mutants as it can. We used MuJava with all the mutation operators (traditional and class-level) it offers.

Some mutants are equivalent to the original program and cannot be killed by any test case. On the other hand, it is possible that the test cases in our test suites may not be able to kill all non-equivalent mutants. This may be due to inadequate test inputs being used in test cases, to oracles not being precise enough, or to code not being executed by test cases $^{7}$. Therefore, when comparing mutant detection rates, we only account for those mutants that can be killed by at least one of the test cases in the original test suite.

MuJava uses two types of mutant operators, class level and method level. We list in Table 4 the mutant operators used in our case studies that resulted in detectable mutants. Appendix E provides, for each of the three case studies, the distribution of mutants per mutation operators. It also provides an analysis of how easy (or hard) it is for the test cases to kills mutants. Variations of number of mutants per mutation operators are observed. This is to be expected since this highly depends on the characteristics of the source code: e.g., a larger number of arithmetic operators in the source code lead to more opportunities to use the AODU mutant operator.

\footnotetext{
${ }^{7}$ For instance, we simplified the Ordered Set state machine diagram and omitted a number of simple methods (without omitting important behavior though), and our test cases therefore do not execute those methods. Since the mutant generation tool we used (MuJava) automatically creates mutants from an analysis of the source code, it created mutants in methods that are never executed by test cases.
} 


\begin{tabular}{|c|c|c|}
\hline $\begin{array}{l}\text { Mutant } \\
\text { Operators }\end{array}$ & Level & Description \\
\hline AODU & method & Arithmetic Operator Deletion - delete basic unary arithmetic operators: $+/-$ \\
\hline AOIS & method & $\begin{array}{l}\text { Arithmetic Operator Insertion - insert short-cut arithmetic operators: op }++,++ \text { op, } \\
\text { op--, --op }\end{array}$ \\
\hline AOIU & method & Arithmetic Operator Insertion - insert unary arithmetic operators: $+/-$ \\
\hline AORB & method & Arithmetic Operator Replacement - replace basic unary arithmetic operators: $+/-$ \\
\hline AORS & method & $\begin{array}{l}\text { Arithmetic Operator Replacement - replace short-cut arithmetic operators: op++, } \\
++ \text { op, op--, --op }\end{array}$ \\
\hline ASRS & method & $\begin{array}{l}\text { Short-Cut Assignment Operator Replacement - replace short-cut assignment } \\
\text { operators with the same kind of short-cut operators: } *=, 1=, \%=, \&=, \mid=, \wedge \text {, } \\
\langle<=,>>=,>>\rangle=\end{array}$ \\
\hline COD & method & Conditional Operator Deletion - delete unary conditional operator: ! \\
\hline COI & method & Conditional Operator Insertion - insert unary conditional operator: ! \\
\hline COR & method & $\begin{array}{l}\text { Conditional Operator Replacement - replace binary conditional operator with other } \\
\text { binary conditional operators: } \& \&, \|, \&, \mid, \wedge\end{array}$ \\
\hline LOI & method & Logical Operator Insertion - insert unary logical operator: $\&,,^{\wedge} \wedge$ \\
\hline ROR & method & $\begin{array}{l}\text { Relational Operator Replacement - Replace relational operator with other relational } \\
\text { operators: }>,>=,<,<=,==, !=\end{array}$ \\
\hline EAM & class & $\begin{array}{l}\text { Accessor method change - change an accessor method name for other compatible } \\
\text { accessor method name. }\end{array}$ \\
\hline JSI & class & $\begin{array}{l}\text { static modifier insertion - add the "static" modifier to change instance variable to } \\
\text { class variable }\end{array}$ \\
\hline PCI & class & $\begin{array}{l}\text { Type cast operator insertion - change the actual type of an object to the parent of } \\
\text { child of the original declared type }\end{array}$ \\
\hline
\end{tabular}

Table 4: Mutation operators used in our case studies

\subsubsection{The Three Case Study Systems}

The first case study is a cruise control system that monitors the speed of a car and allows the driver to set the speed, to cruise, to cancel cruising, ... The second case study is an Ordered Set system, i.e., a data structure with a state-based behavior, that has the capability to resize itself when more room is required to add elements. The third case study is a VCR system that allows a user to play a tape, go forward, backward, ... These three systems were chosen because they have various complexities (source code, test suites, data flow information), as summarized in Table 5. Table 5 shows the number of (un-commented) lines of Java code, the number of test cases and transitions in all the test cases, and the number of data flow elements to cover when using the all-definitions and the all-DU pairs criteria. It also reports on the number of mutants we use for each case study, also illustrating that many mutants are "very hard" or "hard" to kill", and that on average, each mutant is killed by $27.4 \%, 27.1 \%$, and $22.1 \%$ of the test cases in the Cruise 
Control, Ordered Set, and VCR test suites, respectively. The cruise control system is the simplest one of the three systems, the VCR system is the most complex one, whereas the ordered set system has an intermediate complexity.

For different problems, our GA application may take different amounts of time to execute. The GA execution time depends on the number of test cases, the number of transitions, the number of data flow elements. When our GA application does GA operations, e.g., selection, crossover or mutation, the number of test cases affects execution time. When our GA application evaluates the objective values, the number of transitions and the number of data flow elements affects execution time. In fact, our GA application spends more time evaluating objective values than performing GA operations. Table 5 mentions two quite different times: when using definition coverage and when using definition-use pairs coverage. Usually, the GA execution based on all-definitions takes the shorter time, and the GA execution based on all-DU pairs takes the longer time (simply because there are many more definition-uses than definitions).

More details on the three case study systems, including class diagrams, state machine diagrams, transition trees, details on mutants can be found in Appendix E. OCL contracts for the three case study systems can be found in Appendix F.

\begin{tabular}{|l|l|l|l|}
\hline & $\begin{array}{l}\text { Cruise Control } \\
\text { System }\end{array}$ & Ordered Set & VCR \\
\hline LOCs & 271 & 242 & 954 \\
\hline Number of Test Cases & 20 & 165 & 1548 \\
\hline Number of Transitions in Test Suite & 72 & 916 & 8622 \\
\hline Number of definitions & 29 & 152 & 184 \\
\hline Number of DU pairs & 90 & 619 & 1219 \\
\hline Number of mutants used & 234 & 575 & 155 \\
\hline Percentage of "hard to kill" mutants & $20.5 \%$ & $4.9 \%$ & $1.3 \%$ \\
\hline Percentage of "very hard to kill" mutants & $0 \%$ & $0.7 \%$ & $2.6 \%$ \\
\hline Average mutation score of test cases & $27.4 \%$ & $27.1 \%$ & $22.1 \%$ \\
\hline $\begin{array}{l}\text { Execution Time (min and max) for one GA } \\
\text { evolution (mins) }\end{array}$ & $5-30$ & $30-90$ & $120-260$ \\
\hline
\end{tabular}

Table 5: Characteristics of the three case studies

\footnotetext{
${ }^{8}$ Mutants that are only killed by less than $1.5 \%$ of the test cases are considered to be "very hard to kill" whereas mutants that are killed by $1.5 \%$ to $5 \%$ of the test cases are considered to be "hard to kill" [52].

${ }^{9}$ The configuration of the workstation we used to execute our GA is the following: dual $3.2 \mathrm{GHz}$ CPUs, $3 \mathrm{G}$ RAM.
} 


\subsubsection{How Mutants Are Detected}

In our case studies, we firstly generate the test oracle. We execute the complete test suite based on the error-free source code and generate the expected SUT state and other expected values of SUT's attributes/associations, which are shown in the state machine diagram, for each transition of each test case. These recorded data becomes the expected data used by the oracle.

When executing test cases on mutants we record the same information, the same way. Then we compare the actual (mutated) SUT states with the expected SUT states, and compare the actual data of SUT attributes/associates with the expected ones for every test case. If the comparison shows any difference between the expected states/data and the actual states/data, then the mutant is detected by the test case that reveals the difference.

Since we record all expected states that a SUT goes through by executing a sequence of transitions, a wrong state due to a seeded mutant can be detected by comparing the actual state with the expected one. In our case studies, the wrong states due to seeded mutants have indeed been detected.

\subsection{Cruise Control System Case Study-Results}

For each GA run, we present the comparison results with data flow coverage rates and mutant detection rates. In all comparison results for all three case studies, "mean_TCS" refers to the average rate (data flow coverage rate or mutant rate) of GA-evolved sequences at each test cost. "min_TCS" and "max_TCS" refer to the value of the worst and best data flow coverage rates/mutant rates at each test cost. These are compared with the mean, minimum and maximum at each test cost from randomly ordered test case sequences, refered to as "mean_random", "min_random" and"max_random", respectively. We use minimum and maximum values to show the variance between the test case sequences.

We discuss each scheme separately in sections 6.2 .1 to 6.2 .4 , respectively, analyze mutant detection in section 6.2.5 and then summarize the results in section 6.2.6. 


\subsubsection{Cruise Control: Optimization Scheme 1-Specifying a Maximum Test Cost}

In the initial run, we specify a high test cost $T$ (71, i.e., $99 \%$ of total cost), to make sure that all solutions will reach $100 \%$ data flow coverage at $T$. Our GA application shortens $T$ to $T^{\prime}=33$ ( $46 \%$ of total cost) for all-definitions and $T^{\prime}=64(89 \%$ of total cost) for all-DU pairs, respectively. (It is more expansive to cover DU-pairs than definitions.) We show the results in Table 6 . We then try two (sets of) GA runs with test cost $T=29$ and $T=36$ ( $40 \%$ and $50 \%$ of total cost) for all-definitions, and two other (sets of) GA runs with test cost $T=58$ and $T=68$ (80\% and $95 \%$ of total cost) for all-DU pairs. For values larger than $T^{\prime}$, our GA application always shortens them to $T^{\prime}$, and we therefore do not show these results. But we provide the GA results with the smaller values than $T$ ' in Table 6.

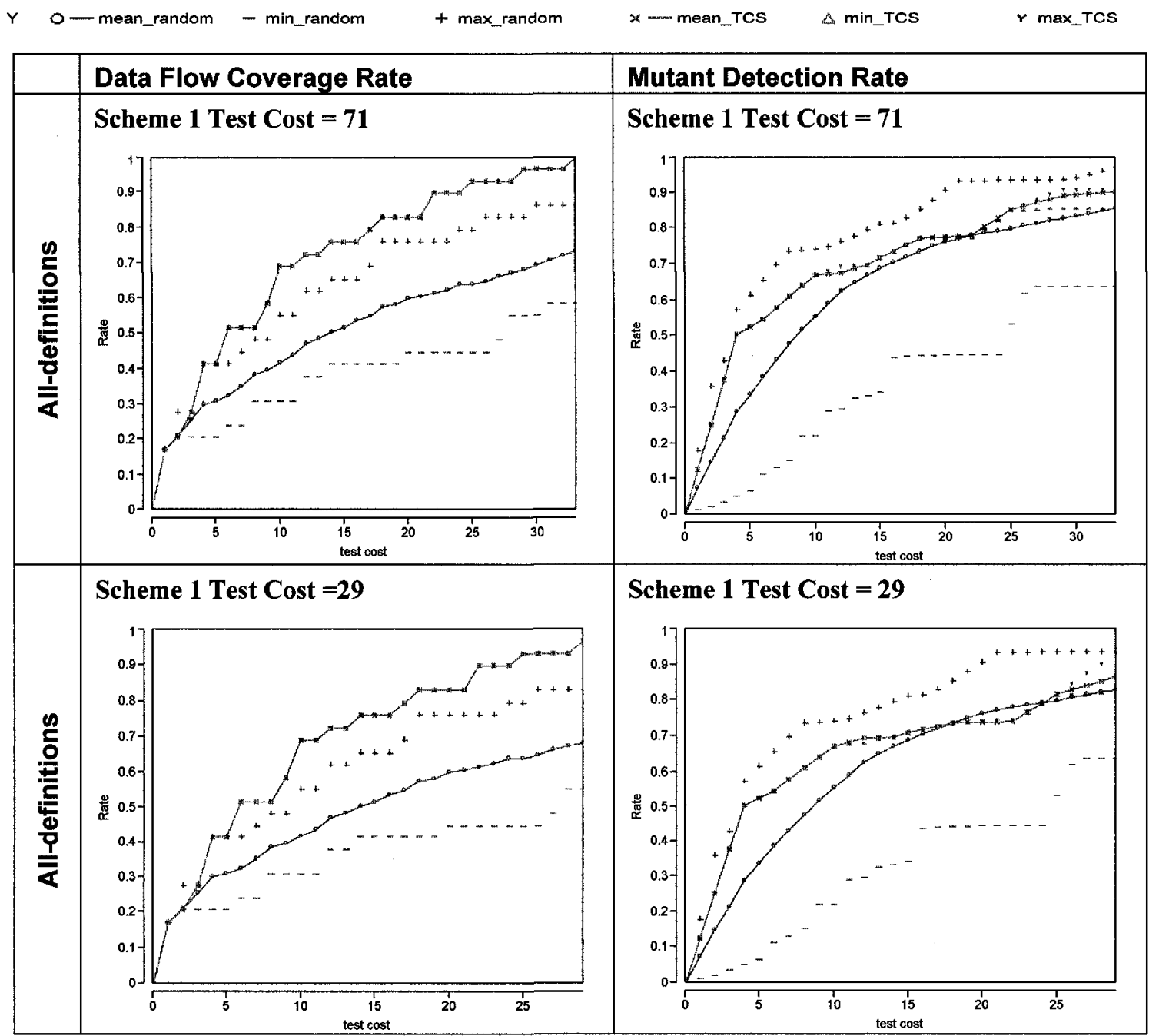




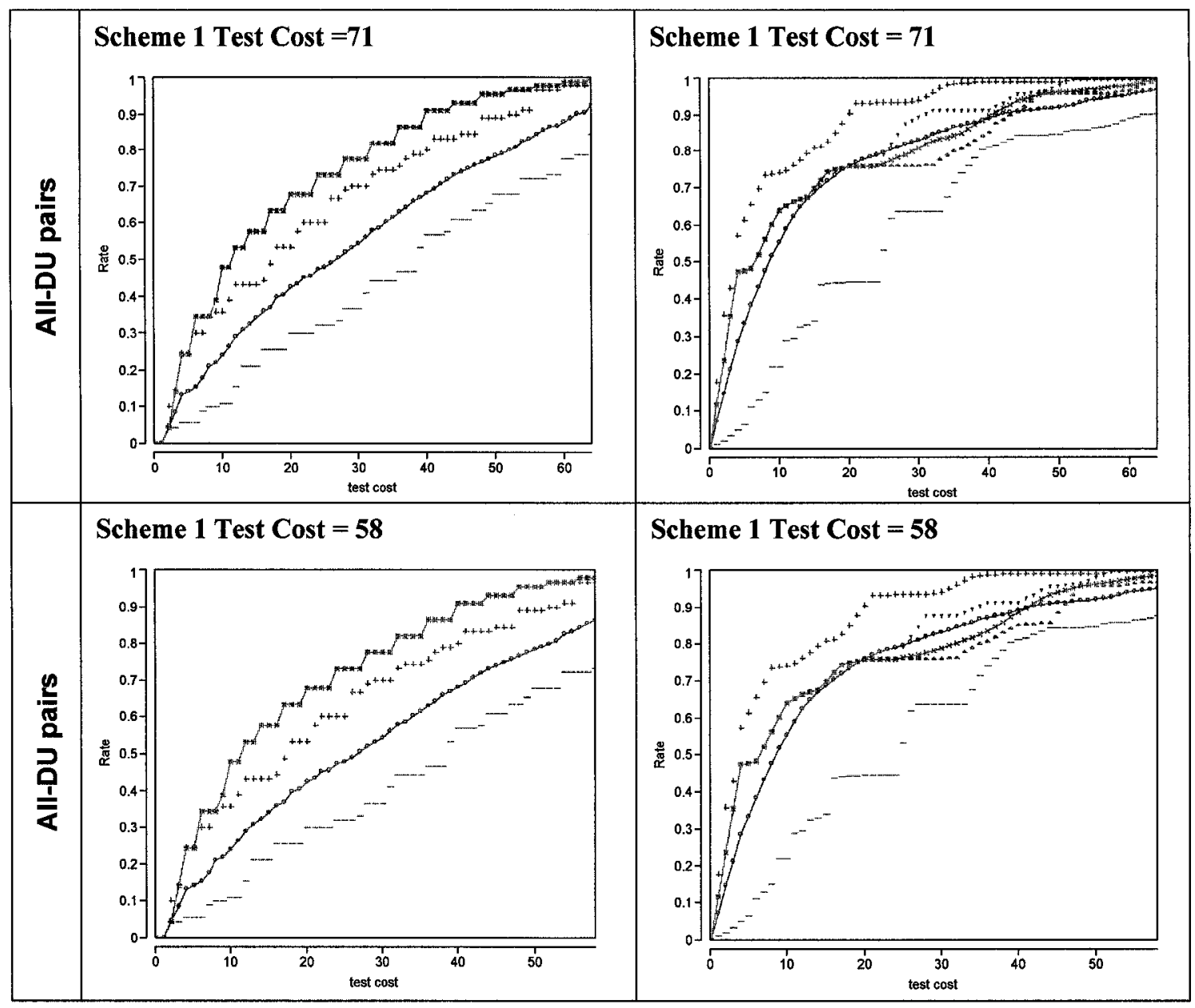

Table 6: Data flow coverage and mutant detection rates of GA-evolved and random sequences (Cruise Control-Scheme 1)

From the comparison reported in Table 6, the GA-evolved sequences increase their data flow coverage rates quickly, which is the intended behavior (i.e., trying to cover more data flow early in the sequence of test cases). For data flow coverage rate, the GAevolved sequences have no variance, since they have the same mean, minimum and maximum values. But the random sequences have a large variance, so their ranges from minimum to maximum are quite big. This variance difference is also noticeable for mutant detection rate curves: low variance with GA-evolved sequences and high variance with random sequences. The GA-evolved sequences detect more mutants on average than the random sequences during the first 20 transitions, with some plateau (e.g., around cost 20)-though the difference is not very large. After test cost 20 , the GA-evolved sequences detect (on average) a similar number of mutants as random sequences, and 
sometimes even fewer mutants than the random sequences. This is further discussed below.

GA-evolved sequences show jumps and small plateaus of data flow coverage. This is due to characteristics of the test cases we used. Recall from section 6.1 that we used Briand et al's algorithm, an adaptation of Binder's algorithm (section 3.1.3), to derive a transition tree and that therefore test case sequences all start at the initial state of the state machine and then correspond to traversals of the state machine graph. Test case sequences therefore share sub-sequences, specifically initial sub-sequences. This is illustrated by the sequences displayed in Table 7: all sequences start with subsequence eIdle@engineon@Running and a large proportion of the sequences start with subsequence aIdle@engineon@Runningeonecruisinge. Therefore, when two test cases that share an initial subsequence are sequenced one after the other by the GA, additional coverage occurs only after the sub-sequence has been executed: there is additional cost, but no additional coverage for the initial, common subsequence, and additional coverage only occurs after the common sub-sequence. Such jumps and plateaus also exist in random sequences. However, because of the randomness of the many sequences we randomly generated, the jumps and plateaus are averaged out and therefore do not appear explicitely in the graphs.

An analysis of data flow coverage and killed mutants for each test case sequence shows that there is no monotonic relationship between the two: increasing coverage of data flow at the model level (recall we use OCL operation contracts), using round trip paths test cases derived from a tree, does not necessarily increases mutant detection. This may seem counter-intuitive. However, having in mind that data flow information is determined at the model level (not the code level), it is not entirely surprising that a method with a complex algorithm (and therefore potentially seeded with many mutants as there are many opportunities to seed faults) will not necessarily be involved in many data flow elements at the model level: models are abstractions. For example, test path (engineOn, engineOff\} covers six definitions and five DU pairs, whereas test path \{engineOn, brake\} covers six definitions and four DU pairs. However since method brake() has a more complex algorithm than method engineoff(), The automatic mutant generation tool, 
MuJava, generates more mutants for brake() (43 mutants) than for engineoff() (30 mutants). So executing \{engineOn, engineOff $\}$ and \{engineOn, brake\} result in similar data flow coverage but the latter can lead to more mutants being killed. This counterintuitive result is also due to the fact that, as discussed earlier, the test cases in the sequences share many transitions. Test case sequences are therefore not optimal in terms of cost. Other test suites, derived using other criteria and techniques would lead to different results.

It is also interesting to note that GA-evolved sequences based on DU-pair coverage show quite some variation (in terms of effectiveness) between cost values 25 and 40 . This is due to many test cases that are equivalent in terms of coverage (the coverage curve does not show variation)-equivalent in terms of number of DU-pairs covered but not necessarily in terms of the actual DU-pairs covered-but have very different mutant detection capabilities (i.e., the sets of DU-pairs covered are in fact different). We observe a smaller variation when using definition coverage after test cost 25: variation occurs less often and to a lesser extent because there are fewer equivalent test cases based on definitions than du-pairs for test costs 25 to 33. Also, recall that results based on definition and du-pairs correspond to different optimizations, i.e., to different runs of the GA with different optimization goals, so that solutions are likely different.

Since the mutant detection rates of the random sequences are so good that random sequences (on average) are even better than some GA-evolved sequences, we specially studied the mutant detection capability of each test case. All test cases, the numbers of covered data flow elements (here we show DU pairs because our GA-evolved sequences are obviously worse than random sequences somewhere) and the numbers of killed mutants are provided in Table 7.

In the total of 20 test cases in the cruise control test set, test cases TC1, TC9, TC11, TC12, TC13, TC14, TC15 and TC19 kill a lot of mutants (resp. 78, 90, 128, 128, 127, 105, 98, 112, out of a total of 234 mutants): The test set of test cases $\{\mathrm{TC} 1, \mathrm{TC} 9, \mathrm{TC} 11, \mathrm{TC} 12, \mathrm{TC} 13$, TC14, TC15, TC19\} kills 200 of the 234 mutants. These test cases involve the most important behaviour of the cruise control system, specifically accelerating. The test set 
\{ $\mathrm{TC11}, \mathrm{TC12}, \mathrm{TC13}, \mathrm{TC14}, \mathrm{TC} 15\}$ is the most interesting subset of all test cases as it kills 180 mutants of the 234 mutants and its most powerful subset \{TC11, TC12, TC13\} kills 171 mutants of the 234 mutants.

Based on the data flow information derived from operation contracts (e.g., all DU pairs), test cases TC11, TC12, TC13, TC14 and TC15 cover relatively large numbers of data flow elements (resp., 17, 14, 17, 22, 18, out of a total of 90), but there are a lot of duplicated data flow elements since they have the same first three transitions. The GA application will therefore always arrange test case $\mathrm{TC} 14$ at the first position since it covers the most data flow elements. This explains why GA-evolved sequences do better on average during the early cost values. But the other test cases TC11, TC12, TC13 and TC15 will be always arranged later since they do not increase data flow coverage a lot (because of redundancies early in the transition sequences) but bring an additional cost (specifically 4) that is higher than other test cases. In other words, because of redundancies in test cases, the GA application selects one of the similar test cases, and "pushes" the others to the end of the sequence, thereby systematically delaying the detection of 28 mutants which could only be detected by subsets of test cases $\{\mathrm{TC1} 1, \mathrm{TC} 12, \mathrm{TC} 13, \mathrm{TC} 15\}$. It seems that GA-evolved sequences are penalized by the many redundancies that exist between round trip path test cases. On the other hand, random test case sequences have more chances of using all those test cases early in the sequences. So in a random test case sequence, if any subset of test cases $\{\mathrm{TC1}, \mathrm{TC}, \mathrm{TC1}, \mathrm{TC12}, \mathrm{TC1}$, TC14, TC15, TC19\} is placed at the beginning of the sequence, its mutant rate is quite close or even better than a GA-generated test case sequence. Because of the big difference in mutant detection between test cases, it is not surprising to see big variations of the mutant rates between random test case sequences. 


\begin{tabular}{|c|c|c|c|}
\hline $\begin{array}{l}\text { Test } \\
\text { Case }\end{array}$ & $\begin{array}{l}\text { Data Flow } \\
\text { Elements }\end{array}$ & \begin{tabular}{|l} 
Killed \\
Mutants
\end{tabular} & Transition Sequences \\
\hline TC1 & 9 & 78 & @idle@engineOn@Running@accelerator@Running \\
\hline TC2 & 4 & 26 & @Idle@engineOn@Running@brake@Running \\
\hline TC3 & 5 & 5 & @Idle@engineOn@Running@engineOff@Idle \\
\hline TC4 & 8 & 10 & @Idle@engineOn@Running@on@Cruising@on@Cruising \\
\hline TC5 & 9 & 23 & @Idle@engineOn@Running@on@Cruising@engineOff@ldle \\
\hline TC6 & 8 & 19 & @Idle@engineOn@Running@on@Cruising@off@Standby@on@Cruising \\
\hline TC7 & 5 & 30 & @Idle@engineOn@Running@on@Cruising@off@Standby@resume@Cruising \\
\hline TC8 & 8 & 33 & @Idle@engineOn@Running@on@Cruising@off@Standby@resume@Cruising \\
\hline TC9 & 13 & 90 & @Idle@engineOn@Running@on@Cruising@off@Standby@accelerator@Standby \\
\hline TC10 & 9 & 17 & @Idle@engineOn@Running@on@Cruising@off@Standby@engineOff@ldl \\
\hline TC11 & 17 & 128 & @Idle@engineOn@Running@on@Cruising@accelerator@Standby@on@cruising \\
\hline TC12 & 14 & 128 & @Idle@engineOn@Running@on@Cruising@accelerator@Standby@resume@cruising \\
\hline $\mathrm{TC} 13$ & 17 & 127 & @Idle@engineOn@Running@on@Cruising@accelerator@Standby@brake@Standby \\
\hline TC14 & 22 & 105 & @Idle@engineOn@Running@on@Cruising@accelerator@Standby@accelerator@Standby \\
\hline TC15 & 18 & 98 & @Idle@engineOn@Running@on@Cruising@accelerator@Standby@engineOff@ldle \\
\hline TC16 & 12 & 44 & @Idle@engineOn@Running@on@Cruising@brake@Standby@on@Cruising \\
\hline $\mathrm{TC} 17$ & 9 & 61 & @Idle@engineOn@Running@on@Cruising@brake@Standby@resume@Cruising \\
\hline TC18 & 12 & 32 & @Idle@engineOn@Running@on@Cruising@brake@Standby@brake@Standby \\
\hline TC19 & 17 & 112 & @Idle@engineOn@Running@on@Cruising@brake@Standby@accelerator@Standby \\
\hline TC20 & 13 & 44 & @Idle@engineOn@Running@on@Cruising@brake@Standby@engineOff@Idle \\
\hline
\end{tabular}

Table 7: Cruise Control Test Set and its data flow/mutant relationship

Last, note that the discussion we just had about mutant rates and the qualitative analysis of some test case sequences and (un-)killed mutants also applies to the other schemes, although we won't systematically report on that when discussing the other schemes.

\subsubsection{Cruise Control: Optimization Scheme 2-Specifying a Cumulative Coverage Rate}

In this optimization scheme, we specify two user expected cumulative coverage rates $(S C): 80 \%$ and $90 \%$ data flow coverage rate for all-definitions and all-DU pairs: Table 8 . For this scheme, it happens that all the GA-evolved sequences take the same test cost to reach $S C$, so we do not observe the (expected) non-monotonic increasing in data flow coverage rates and mutant detection rates of the GA-evolved sequences after cost $T_{\text {shortest }}$ as discussed in sections 6.1.2.1 and 6.1.2.2. The (cumulative) data flow coverage rates and mutant detection rates of random sequences appear non-monotonic after a certain (cost) threshold $T_{\text {shortest }}$, as already explained in section 6.1.2.1: $T_{\text {shortest }}$ is the shortest test cost of GA-evolved sequences to reach $S C$. 


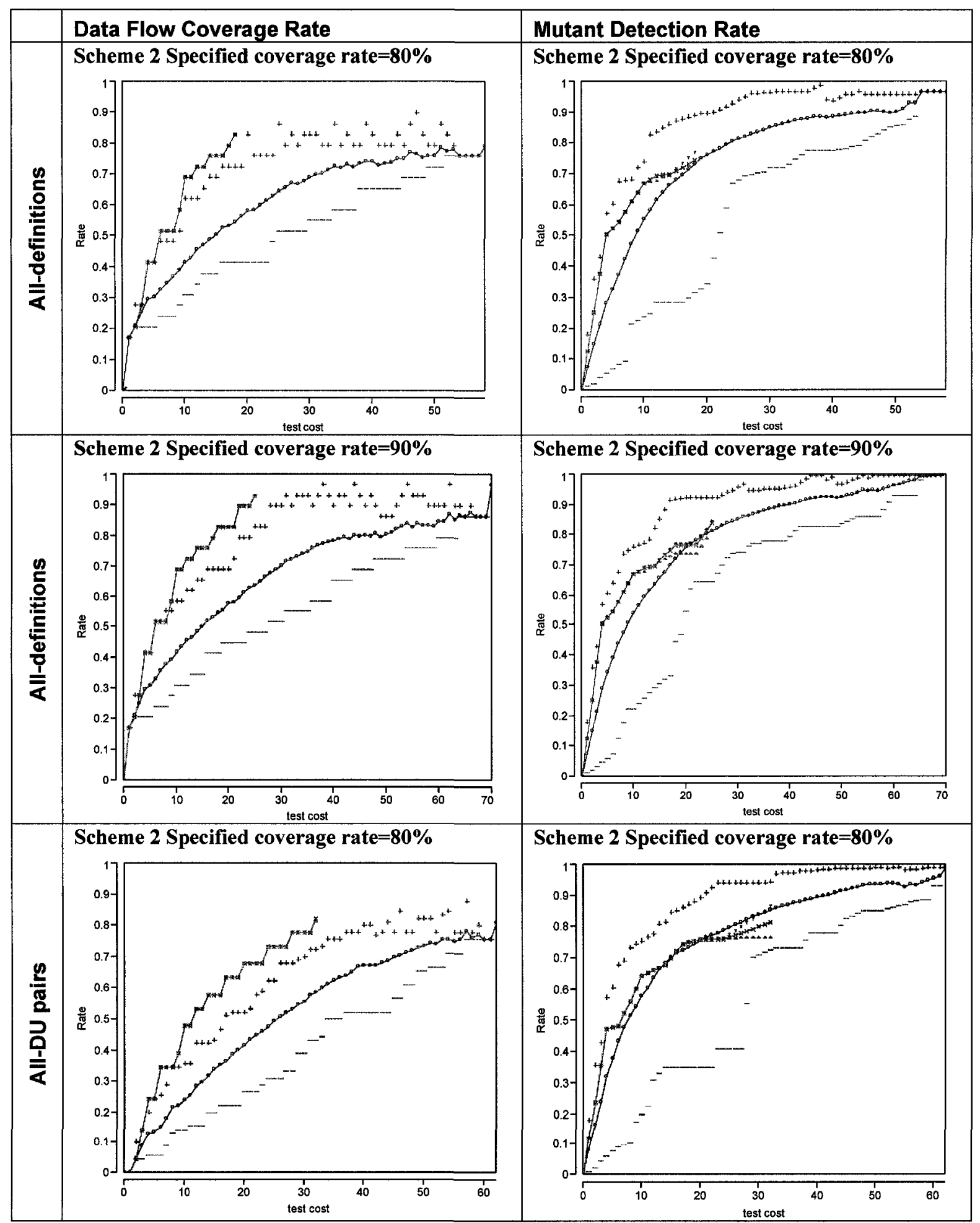




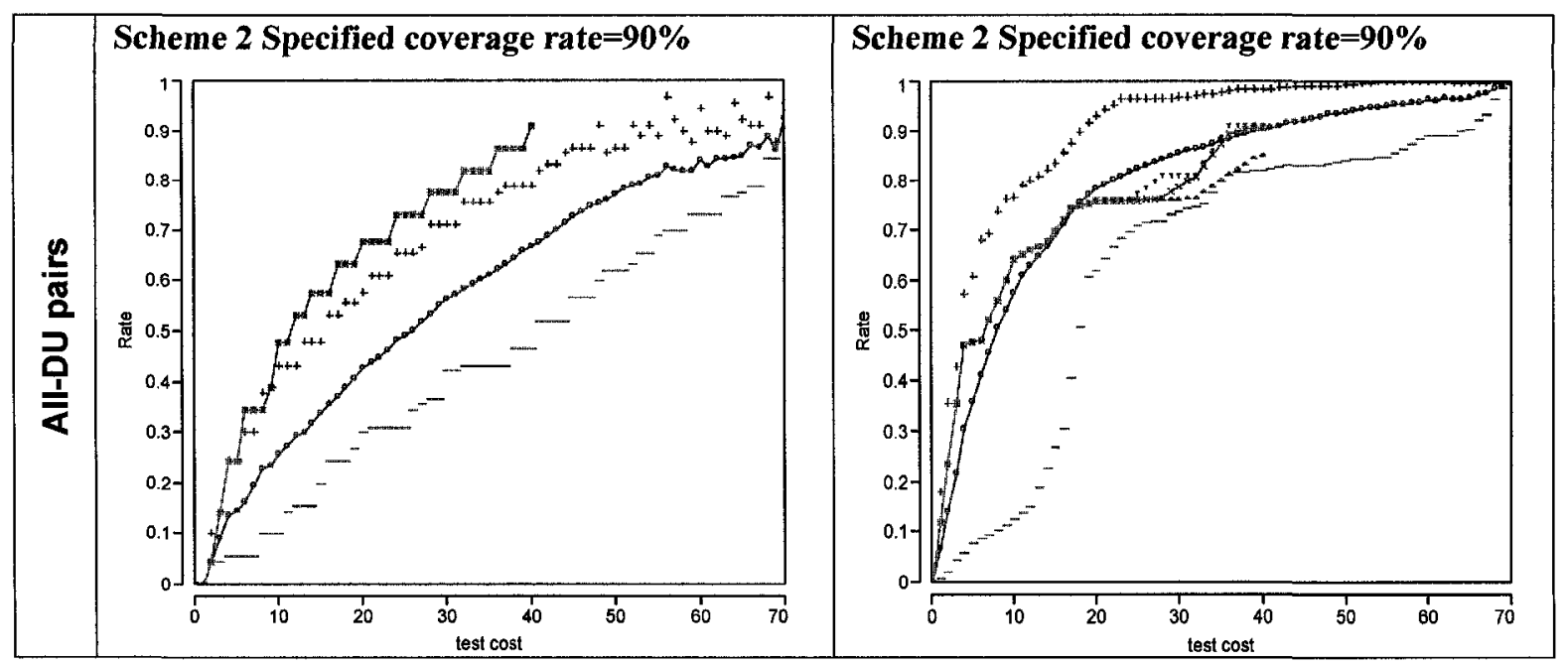

Table 8: Data flow coverage and mutant detection rates of GA-evolved and random sequences (Cruise Control-Scheme 2)

From Table 8, the GA-evolved sequences based on all-DU pairs and all-definitions increase their data flow coverage rate quickly and reach the data flow coverage much earlier than random sequences. The GA-evolved sequences reach $80 \%$ and $90 \%$ of all definitons at test costs 18 and 25, respectively, and $80 \%$ and $90 \%$ of all DU pairs at test costs 32 and 40, respectively. The random sequences reach $80 \%$ and $90 \%$ of all definitions at test costs in ranges $[21,58]$ and $[30,70]$, respectively, and $80 \%$ and $90 \%$ of all DU pairs at test costs in ranges $[32,61]$ and $[47,70]$, respectively. From the analysis of data flow coverage rates, the GA-evolved sequences have no variance but the random sequences have a large variance.

For the mutant detection rate, in general, the GA-evolved sequences are better than random sequences at the beginning; however, there is no significant difference after test cost 20. But the variance of the GA-evolved sequences is much smaller than the variance of the random sequences. Similarly to the previous scheme, and for reasons similar to the ones provided earlier, GA-evolved sequences do better on average during the early cost values.

Similarly to scheme 1, mutant detection shows more variation when using du-pairs than when using definitions, the definition curve seems to be higher than the du-pair curve (compared to the same random curves) for the same test cost. 


\subsubsection{Cruise Control: Optimization Scheme 3-Specifying 100\% Cumulative Coverage Rate and Maximum Available Test Cost}

In this optimization scheme, we do not specify any user expected data. This scheme simply uses $100 \%$ cumulative coverage rate and $100 \%$ of total transitions. The comparison result is given in Table 9, where $S C$ refers to the user specified coverage rate and $T$ refers to the user specified test cost ( $100 \%$ or 1.0 , and $100 \%$ or 72 , respectively).

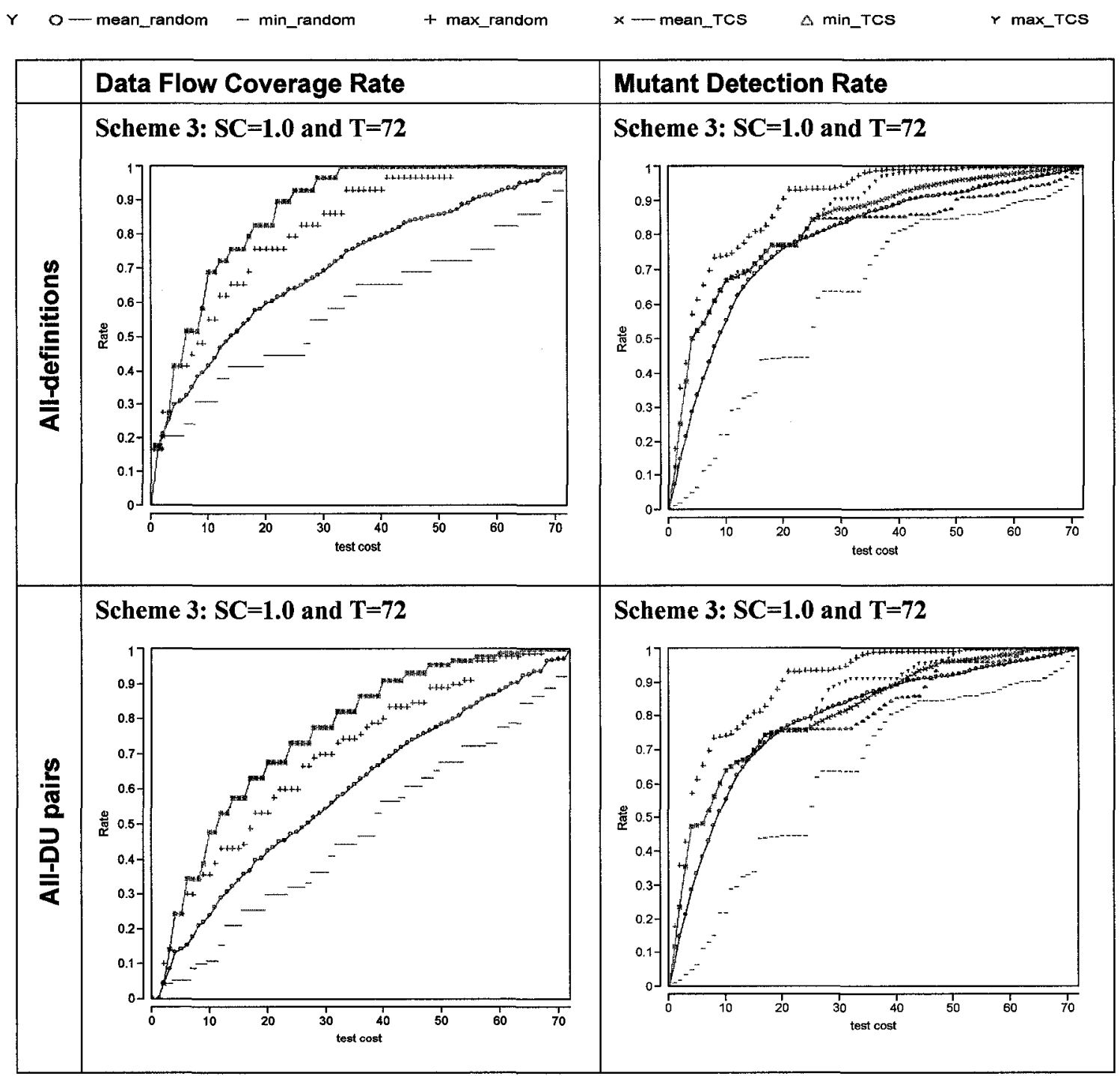

Table 9: Data flow coverage and mutant detection rates of GA-evolved and random sequences (Cruise Control-Scheme 3) 
With $100 \%$ of total transitions and $100 \%$ data flow coverage rate, we observe similar results as the other optimization schemes: the GA-evolved sequences increase their data flow coverage rates quicker than the random sequences; the GA-evolved sequences have no variance in data flow coverage rate but the random sequences have large variances; the GA-evolved sequences have better mutant rates than the random sequences at the beginning; the GA-evolved sequences have mutant rates similar to the random sequences later in the sequences. The average mutant rate of the GA-evolved sequences reaches $87.4 \%$ when the average data flow coverage rate based on all-definitions reaches $100 \%$; the average mutant rate of the GA-evolved sequences reaches $100 \%$ when the average data flow coverage rate based on all-DU pairs reaches $100 \%$.

It is interesting to note that GA-evolved sequences based on all-definitions coverage show some variation in effectiveness after cost value 25 and quite large variation after cost value 33 . The large variation after cost value 33 is due to the fact that once at cost 33 , definition coverage has reached $100 \%$. Since our fitness functions do not account for what happens once $100 \%$ coverage has been reached, all test cases after cost 33 are equivalent to the genetic algorithm (any change to the test cases sequences after cost 33 does not help improve fitness values). Since those changes are random (because they are due to cross-over and mutation), effectiveness shows a lot of variation. The variation appears to even start before cost 33: in fact around cost 25 because of the existance of equivalent test cases in terms of data flow coverage (recall the discussion in section 6.2.1).

One additional observation we can make is that, based on this case study system and this scheme, GA-evolved sequences based on all-definitions outperforms GA-evolved sequences based on all-DU pairs in terms of effectiveness at killing mutants. This can be observed when comparing GA-evolved curves to the average random curve. It appears from Table 9, that the all-definitions curve is usually above the average random curve whereas the all-DU pairs curve is not: for instance, it is almost identical to the random average at cost 15 and goes below (worse) the average random curve from cost 20 to 35 . However, for different data flow criteria, at the test cost where the GA-evolved sequences reach their coverage goals ( 33 and 64 respectively), the all-DU pairs curve reaches $100 \%$ 
mutation rate whereas the all-definitions curve does not. In fact, the GA-evolved sequences based on all-definitions reach $100 \%$ mutant rate later than the ones based on all-DU pairs. This confirms observations that have already been made in [5]: (1) covering all the definitions allows to detect a large proportion of the mutants (87\%), and this is easier than covering du-pairs, (2) covering definitions is not sufficient to kill some mutants, which can only be killed by covering specific du-pairs (this was already observed in [5]).

\subsubsection{Cruise Control: Optimization Scheme 4-Specifying a Maximum Test Cost and a Minimum Cumulative Coverage Rate}

In this optimization scheme, we need to specify a maximum test cost and a minimum cumulative coverage rate. Since our GA application can heuristically shorten the test cost to a value that satisfies the minimum cumulative coverage rate, we simply specify relatively large test cost and data flow coverage rates $S C$ of $80 \%$ and $90 \%$. Empirically, given our understanding of the system under test, we then specify test cost values of 58 and 65 (around $80 \%$ and $90 \%$ of total cost) for $S C=80 \%$ and $S C=90 \%$, respectively, to make sure a large number of solutions in the initial population can reach the minimum $S C$ at the end of test case sequences. (Note that we did not use the full cost since this would then be equivalent to scheme 2.) We present the comparison results based on the initial user input data in Table 10. 


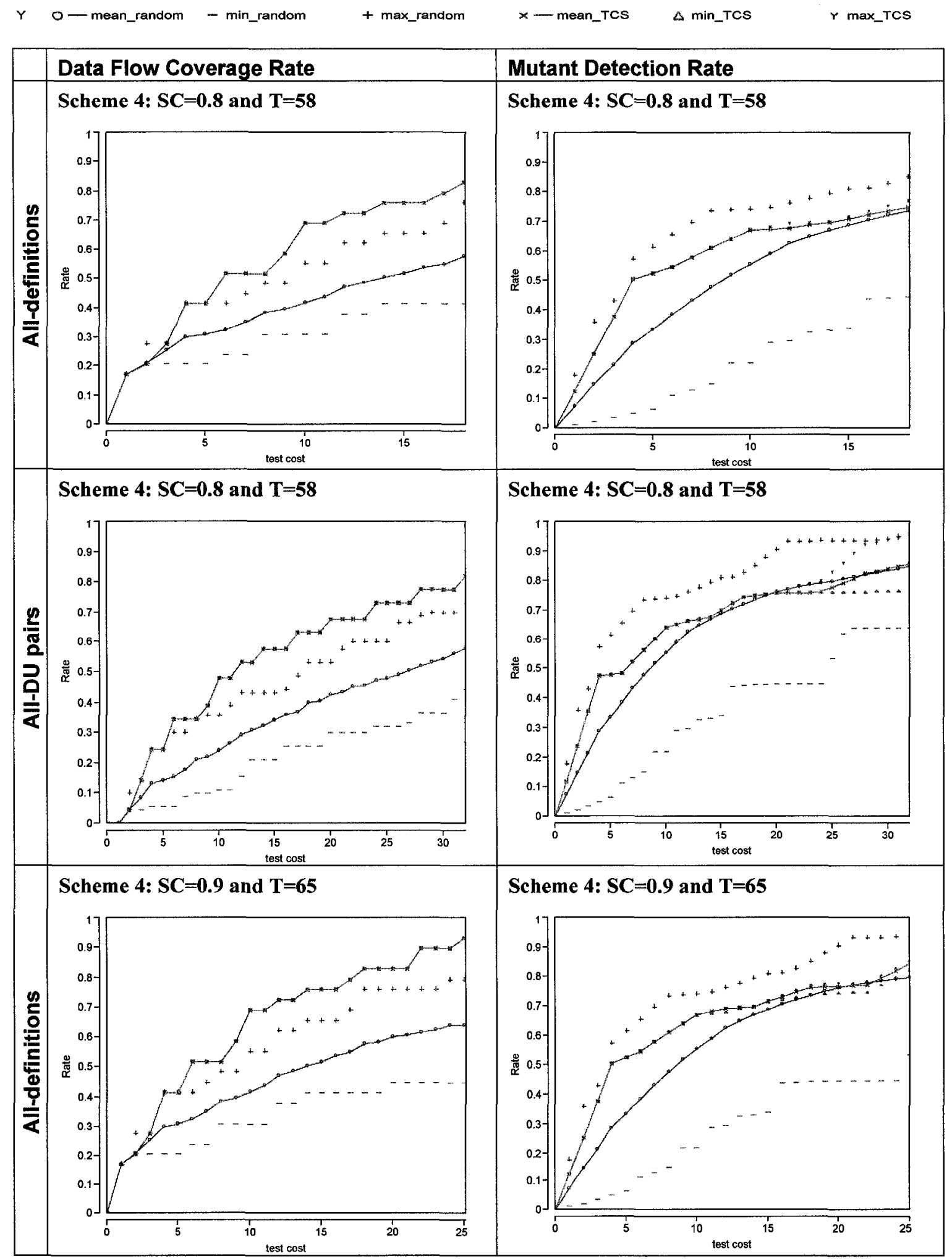




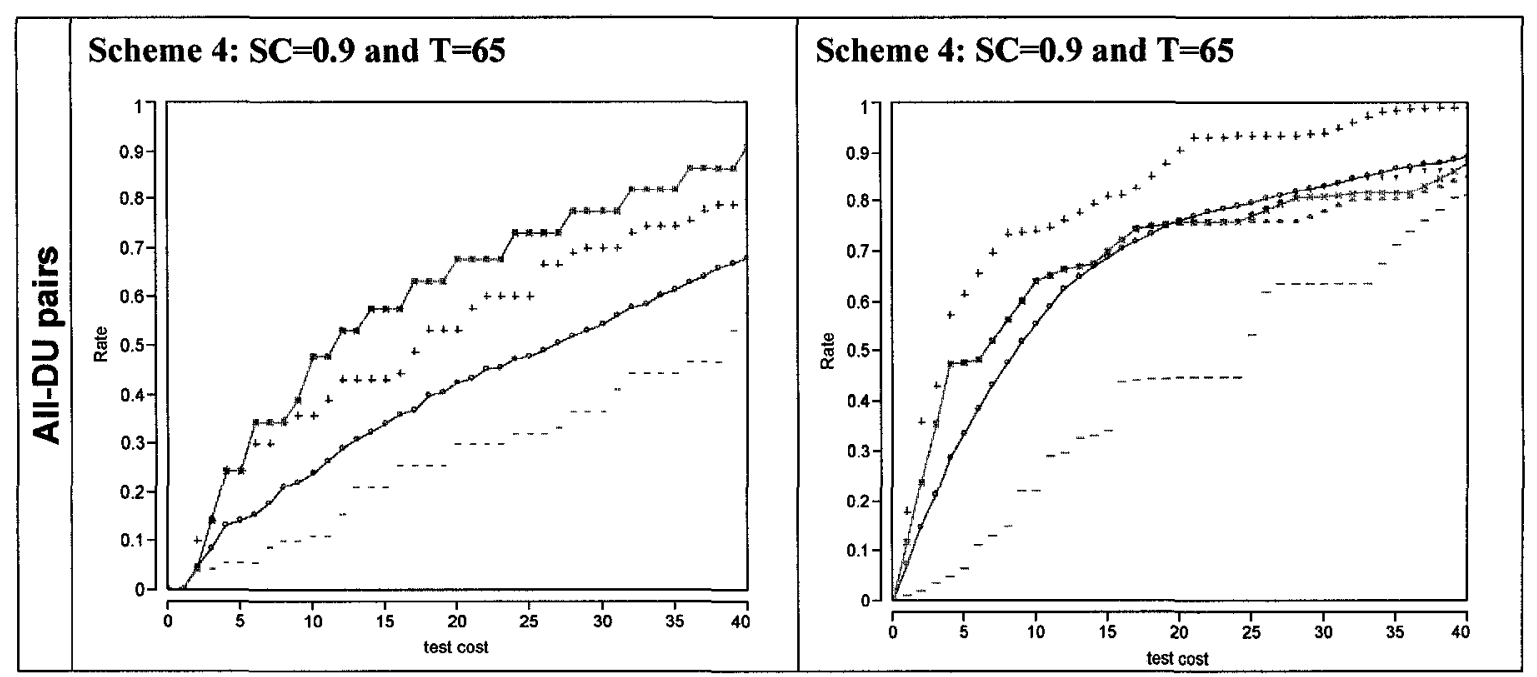

Table 10 : Data flow coverage and mutant detection rates of GA-evolved and random sequences (Cruise Control-Scheme 4)

From Table 10, we can see that the data flow coverage of GA-evolved sequences increases faster than the one of random sequences and reaches the specified data flow coverage rate with less test costs than the user-specified test cost. To cover $80 \%$ and $90 \%$ of all definitions, GA-evolved sequences need sequences of cost 18 and 25 respectively. To cover $80 \%$ and $90 \%$ of all DU pairs, GA-evolved sequences need sequences of cost 32 and 40 respectively. The GA-evolved sequences have no variance in the data flow coverage rate but the random sequences have much large variances.

For the mutant detection rate, the GA-evolved sequences have better average values for cost values up to 20 , but similar and even lower values after that. But the GA-evolved sequences have much smaller variances in the mutant detection rates. This has already been discussed in Section 6.2.1.

One can note that DU-pair coverage does not increase right away: one needs to wait for cost value two to see an increase. This was also the case in previous schemes but less legible because of the unit on the $\mathrm{x}$-axis. This is to be expected since to cover a du-pair one needs to reach a definition and then a use, which in our case means that we need to cover at least two transitions. 
Since our GA application shortens $T$ to $T$ ' for all-definitions and all-DU pairs, we re-run the GA with slightly smaller and larger values of $T^{\prime}$, as discussed in section 6.1.2.3. For all-definitions, we therefore specify test costs 14 and 22 for $\mathrm{SC}=80 \%$ (20\% and $30 \%$ of total cost, smaller and larger values than 18), and 22 and 29 for $\mathrm{SC}=90 \%$ (20\% and $30 \%$ of total cost, smaller and larger values than 25). Similarly, for all-DU pairs, we specify test costs $29(40 \%)$ and $36(50 \%)$ for $\mathrm{SC}=80 \%$, and $36(50 \%)$ and $43(60 \%)$ for $\mathrm{SC}=90 \%$. Since the results of the larger values are very similar to the initial runs (i.e, the GA returns to the same $T$ ' values), we do not present those results here. Table 11 presents the comparison results based on the combination of the smaller $T^{\prime}$, values and the two cumulative coverage rates of $80 \%$ and $90 \%$.

Y O-mean_random - min_random + max_random $W_{\text {man mean_TCS }}^{A}$ min_TCS $r$ max_TCS

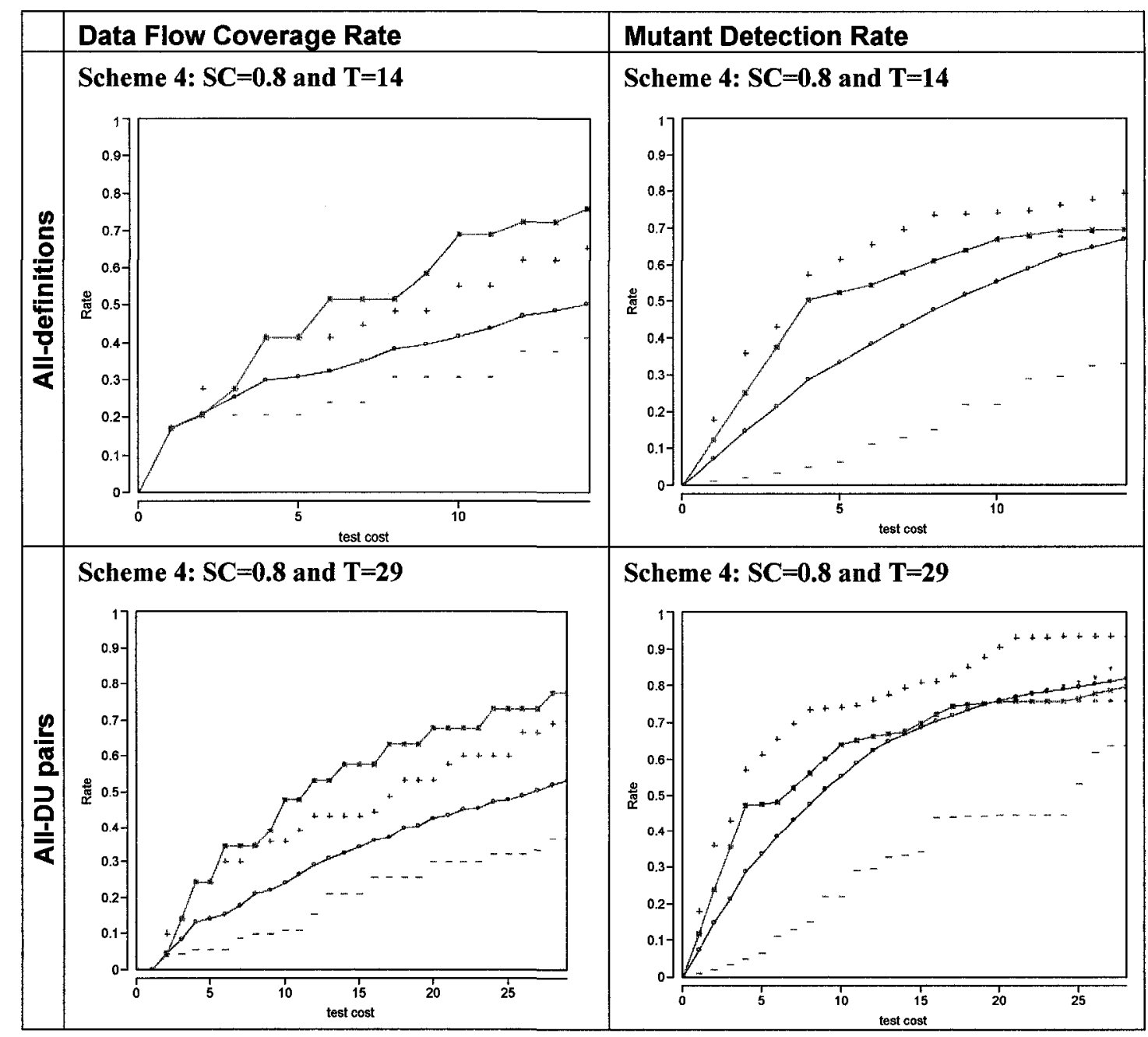




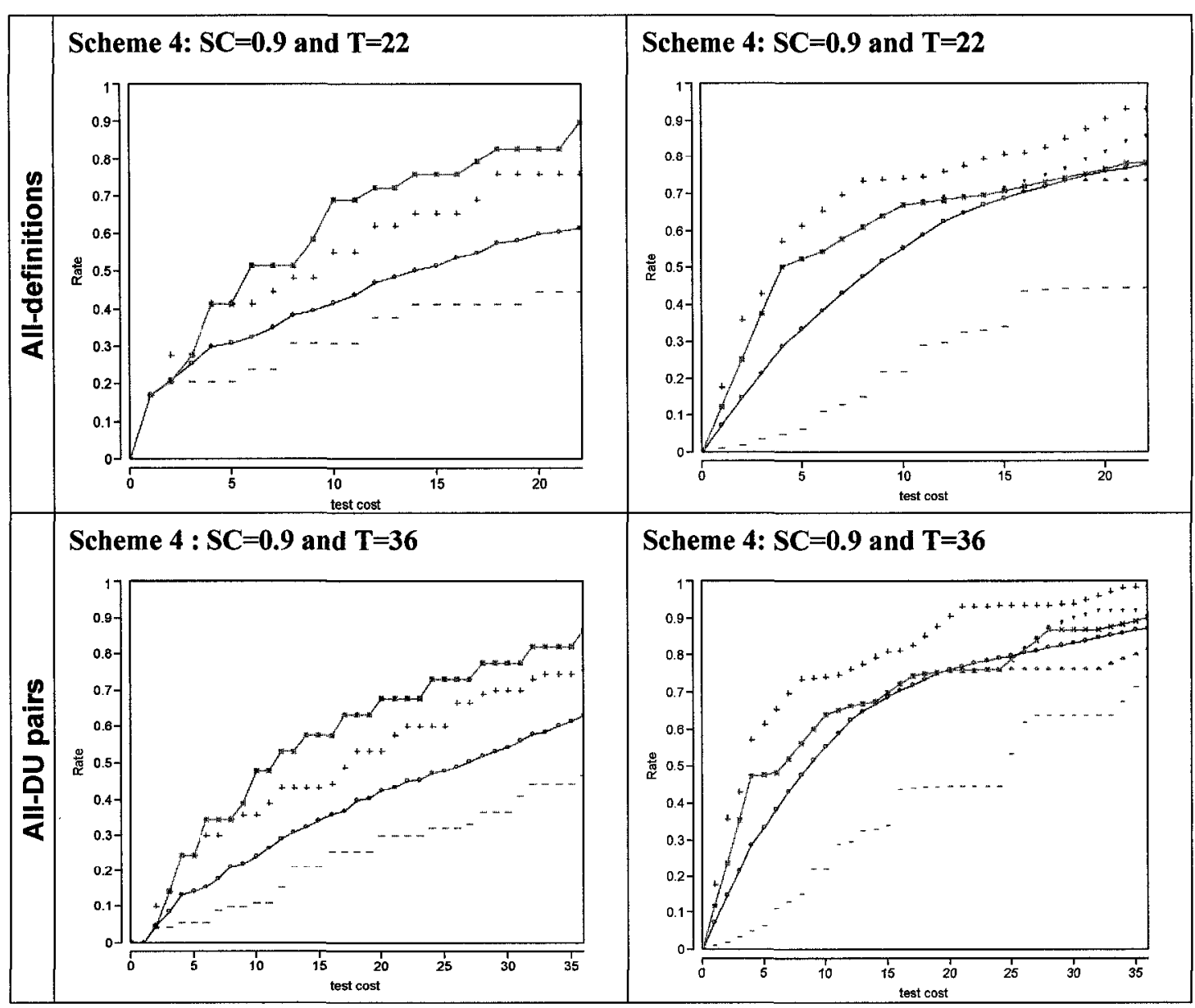

Table 11: Data flow coverage and mutant detection rates of GA-evolved and random sequences with new Ts (Cruise Control-Scheme 4)

For the new test costs 14 and 22, the GA-evolved sequences coud not cover $80 \%$ and $90 \%$ of all definitions, respectively. They are only enough to cover $76 \%$ and $89.7 \%$ of all definitions on average, respectively. For the new test costs 29 and 36, the GA-evolved sequences coud not cover $80 \%$ and $90 \%$ of all DU pairs. They are only enough to cover $77.8 \%$ and $86.7 \%$ of all DU pairs, respectively.

Similarly to results reported earlier, GA-evolved sequences increase their data flow coverage rates faster than random sequences and have no variance whereas random sequences have a lot of variances. Also, GA-evolved sequences have better mutant rates and much less variance than the random sequences at the beginning of the sequences, but they tend to have similar mutant rates to the random sequences later in sequences. 


\subsubsection{Cruise Control: Further studying mutant rates}

In the above four optimization schemes, we observed that GA-evolved sequences achieve similar mutant rates as random sequences after the first 20 test costs. In section 6.2.1, we already studied the number of killed mutants by each test case, which revealed the significant difference of mutant detection capability of those test cases. We identified a set of test cases, which we referred to as the powerful test set $\{\mathrm{TC1}, \mathrm{TC}, \mathrm{TC1} 1, \mathrm{TC} 12$, TC13, TC14, TC15, TC19\} that kills 200 of the 234 mutants. We argued that any random sequence picking up one or more test cases from the powerful test set would increase its mutant rate sharply. This is the main reason we identified that explains that random sequences can outperform the GA-evolved sequences in terms of mutant rate. We here go further in our analysis and investigate what makes this set so powerful.

The main reason is that the mutant distribution over methods in the source code is unbalance: a large number of mutants (one would say too many mutants) are inserted in the code that implements the "accelerating" behavior (as specified in the state machine): the distribution of mutants per method in Appendix E shows an average of 11 mutants per method and a standard deviation of 13 (maximum and minimum number of mutants per method are 56 and 1 respectively). As a result, test cases involving the "accelerating" behavior have a better chance to (and they do) kill a large proportion of mutants. The unbalanced mutant distribution causes significant differences in mutant detection capabilities among the 20 test cases in the complete test suite.

To further investigate the impact of this balancing of mutants in methods on our results, we tried to have a more balanced distribution of mutants per method. To balance the mutant distribution, we could add more mutants associated with the other behaviors/methods (other than "accelerating"), or we could reduce the number of mutants associated with the behavior "accelerating". Since MuJava already seeds as many mutants as possible, adding more mutants may be very difficult. So we try to reduce the number of mutants associated with the behavior "accelerating". We try to balance the number of mutants per behavior specified in the state machine. 
However, because of the many classes and methods that implement the state based behavior of the state machine, it is difficult to identify whether a mutant is seeded in the code that implements a behavior specified in the state machine. So we start by reducing the number of killed mutants by test cases in the powerful test set $\{\mathrm{TC} 1, \mathrm{TC}, \mathrm{TC} 11, \mathrm{TC} 12$, TC13, TC14, TC15, TC19\}, which kill mutant 108 on average ${ }^{10}$, by removing some of the mutants they all kill. Since the remaining test cases, namely, TC2, TC3, TC4, TC5, TC6, TC7, TC8, TC10, TC16, TC17, TC18, and TC20 kill 28.7 mutants on average (based on the data in Table 7), we then try to reduce the number of mutants killed on average by the test cases in the powerful test set to a value close to that of the other test cases. After removing some mutants, the powerful test cases kill on average between 40 to 50 mutants, and all test cases kill on average between 30 to 35 mutants.

When we remove mutants, firstly we remove 60 mutants only detected by test cases in set $\{\mathrm{TC} 1, \mathrm{TC} 9, \mathrm{TC} 11, \mathrm{TC1}, \mathrm{TC} 13, \mathrm{TC} 14, \mathrm{TC} 15, \mathrm{TC} 19\}$. Then we remove 3 mutants only detected by test cases in set $\{\mathrm{TC1} 1, \mathrm{TC1}\}, 2$ mutants only detected by test cases in set $\{\mathrm{TC} 11, \mathrm{TC} 13\}$, and 1 mutant only detected by test cases in set $\{\mathrm{TC}, \mathrm{TC} 11, \mathrm{TC} 12, \mathrm{TC} 13\}$. To achieve our goal, we also remove 11 mutants only detected by test cases in set \{TC11, TC12, TC16, TC17\}. When we remove mutants, we do not remove any of the hard mutants, i.e., ones which can only be killed by one test case. In the end, we have 157 mutants left and the number of killed mutants on average is 33.6 for all the test cases. The new table with all test cases, the numbers of covered data flow elements and the numbers of killed mutants is provided in Table 12 (to be compared with Table 7). From the number of killed mutants by each test case, we do not see the significant difference between test cases any more. Appendix E shows the new distribution of seeded mutants per method in the source code: average and standard deviations of 7 (minimum and maximum numbers of mutants per method of 0 and 22 respectively).

\footnotetext{
${ }^{10}$ The definition of the number of killed mutants on average is: $K m O A=\sum_{i=o}^{N} K m(i) / N$, where $N$ is the total number of test cases and $K m(i)$ is the number of killed mutant by test case $i$.
} 


\begin{tabular}{|c|c|c|c|}
\hline $\begin{array}{l}\text { Test } \\
\text { Case } \\
\end{array}$ & $\begin{array}{l}\text { Data Flow } \\
\text { Elements }\end{array}$ & $\begin{array}{l}\text { Killed } \\
\text { Mutants }\end{array}$ & Transition Sequences \\
\hline TC1 & 9 & 18 & @Idle@engineOn@Running@accelerator@Running \\
\hline TC2 & 4 & 26 & @Idle@engineOn@Running@brake@Running \\
\hline TC3 & 5 & 5 & @Idle@engineOn@Running@engineOff@ldle \\
\hline TC4 & 8 & 10 & @Idle@engineOn@Running@on@Cruising@on@Cruising \\
\hline TC5 & 9 & 23 & @Idle@engineOn@Running@on@Cruising@engineOff@Idle \\
\hline TC6 & 8 & 19 & @Idle@engineOn@Running@on@Cruising@off@Standby@on@Cruising \\
\hline TC7 & 5 & 30 & @Idle@engineOn@Running@on@Cruising@off@Standby@resume@cruising \\
\hline TC8 & 8 & 33 & @ldle@engineOn@Running@on@Cruising@off@Standby@resume@cruising \\
\hline TC9 & 13 & 29 & @Idle@engineOn@Running@on@Cruising@off@Standby@accelerator@Standby \\
\hline TC10 & 9 & 17 & @Idle@engineOn@Running@on@Cruising@off@Standby@engineOff@ldl \\
\hline $\mathrm{TC} 11$ & 17 & 51 & @Idle@engineOn@Running@on@Cruising@accelerator@Standby@on@Cruising \\
\hline TC12 & 14 & 53 & @Idle@engineOn@Running@on@Cruising@accelerator@Standby@resume@Cruising \\
\hline TC13 & 17 & 64 & @Idle@engineOn@Running@on@Cruising@accelerator@Standby@brake@Standby \\
\hline TC14 & 22 & 45 & @Idle@engineOn@Running@on@Cruising@accelerator@Standby@accelerator@Standby \\
\hline TC15 & 18 & 38 & @Idle@engineOn@Running@on@Cruising@accelerator@Standby@engineOff@Idle \\
\hline TC16 & 12 & 33 & @Idle@engineOn@Running@on@Cruising@brake@Standby@on@Cruising \\
\hline TC17 & 9 & 50 & @Idle@engineOn@Running@on@Cruising@brake@Standby@resume@Cruising \\
\hline TC18 & 12 & 32 & @Idle@engineOn@Running@on@Cruising@brake@Standby@brake@Standby \\
\hline TC19 & 17 & 52 & @Idle@engineOn@Running@on@Cruising@brake@Standby@accelerator@Standby \\
\hline $\mathrm{TC} 20$ & 13 & 44 & @Idle@engineOn@Running@on@Cruising@brake@Standby@engineOff@Idle \\
\hline
\end{tabular}

Table 12: Cruise Control Test Set and its data flow/mutant relationship after

\section{balancing mutant distribution}

With the 157 mutants, we re-do the comparison between GA-evolved sequences of random sequences of section 6.2 .3 (i.e., scheme 3 ) as an example. Table 13 shows the mutant rates of the GA-evolved sequences and the random sequences based on the original 234 mutants, as well as the mutant rates of the same sequences based on the 157 mutants. 


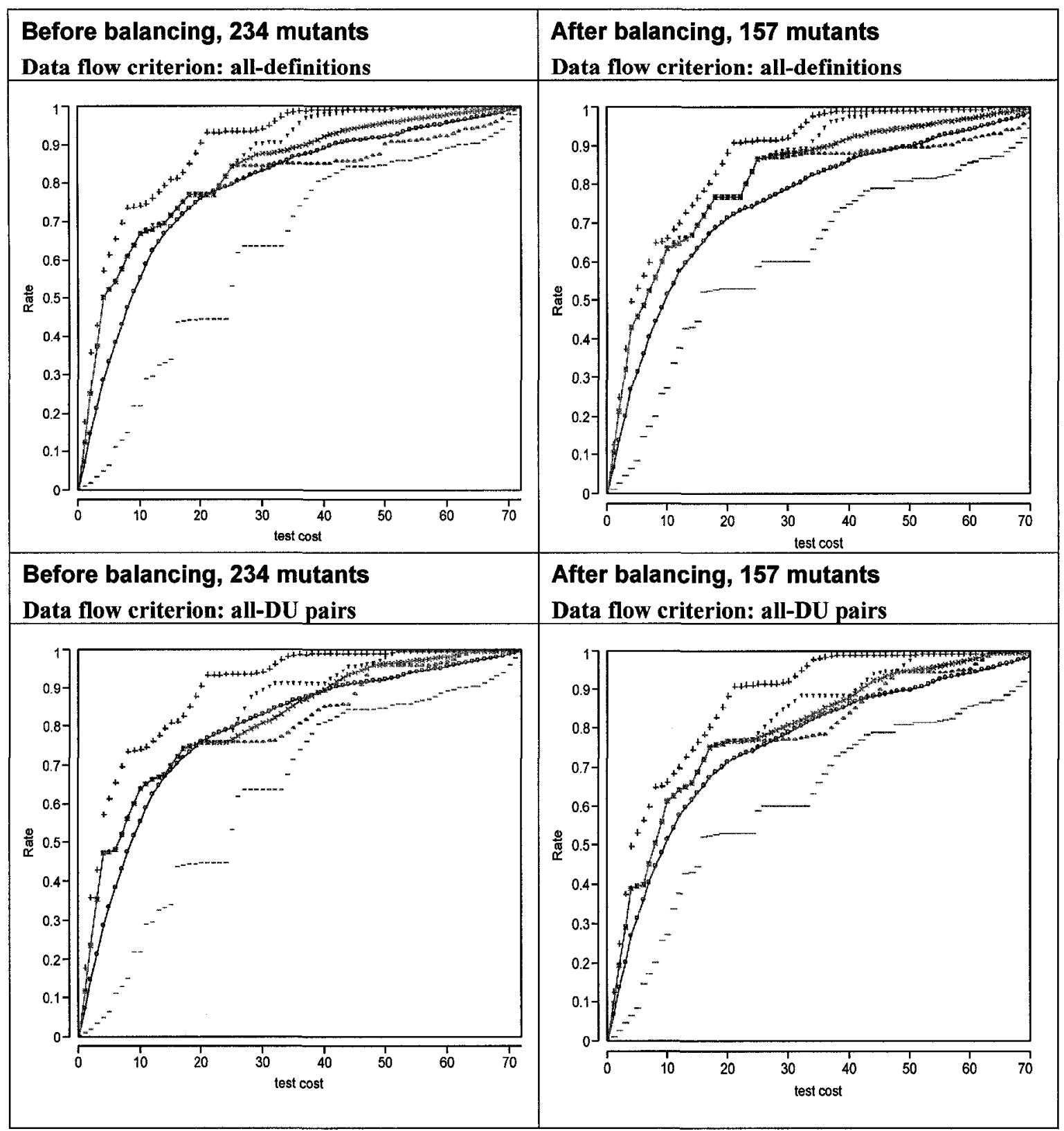

Table 13: Data flow coverage and mutant detection rates of GA-evolved and random sequences with different mutant sets (Cruise Control-Scheme 3)

Using the all-definitions criterion, we observe that with 234 mutants, the GA-evolved sequences usually detect more mutants than the random sequences, but between test costs 21 to 25 , the GA-evolved sequences obtain similar mutant rates as the random sequences (on average). With the 157 mutants, the GA-evolved sequences always detect more mutants $(10 \%$ on average) than the random sequences. Based on all-DU pairs, with 234 
mutants, the GA-evolved sequences detect more mutants than random sequences from test cost 0 to test cost 20 , but between test costs 20 and 40, the random sequences detect more mutants than the GA-evolved sequences. With 157 mutants, the GA-evolved sequences always detect more mutants (5\% on average) than random sequences except between test costs 25 and 40 where the GA-evolved sequences detect similar mutants as the random sequences (on average). This analysis confirms the general observations we made in previous sections. As well, we observe that when balancing the mutant distribution, similarly to what we observed for scheme 3 , the GA-evolved sequences based on all-definitions outperform the GA-evolved sequences based on all-DU pairs (in fact, going through all GA-evolved sequences from different optimization schemes in this case study, we always notice the same trend). But it takes specific du-pairs to kill some mutants, which explains why the DU-pair mutant rate reaches $100 \%$ a bit faster than the definition mutant rate. This analysis indicates that the fact that GA-evolved and random sequences were so close to one another in previous analysis was indeed due to unbalanced seeding of mutants in the code. Also, a fewer number of mutants (157 instead of 234) leads to a smaller effectiveness variance of random sequences - which was to be expected. Given that the curve of the maximum value does not represent an actual sequence of test cases, this confirms that GA-evolved sequences do perform better than random sequences.

\subsubsection{Cruise Control: Summary of Results}

In our study, we find that the data flow coverage rates of GA-evolved sequences are better on average than the ones of random sequences, but the mutant detection rates of GA-evolved sequences are not necessarily better on average than the ones of the random sequences. For the data flow coverage rate, all GA-evolved sequences obtained in one GA run have the exactly same data flow coverage rate curves (i.e., they are equivalent in phenotype). But these sequences have slightly different test case sequences (i.e., they are different in genotype). For the mutant detection rate, we observe that GA-evolved sequences have very little variance, whereas random sequences have large variances. This is due to the fact that GA-evolved sequences look very similar. Let us take a representative example, using criterion all-definitions and scheme 3 . We report below 
three randomly picked up sequences from the non-dominated solutions. (The format in the following three test case sequences shows test case numbers and incremental costs: e.g., 19L4-1L 6 means the first test case is TC19 ending at test cost 4 , and the second test case is TC1 ending at test cost 6).

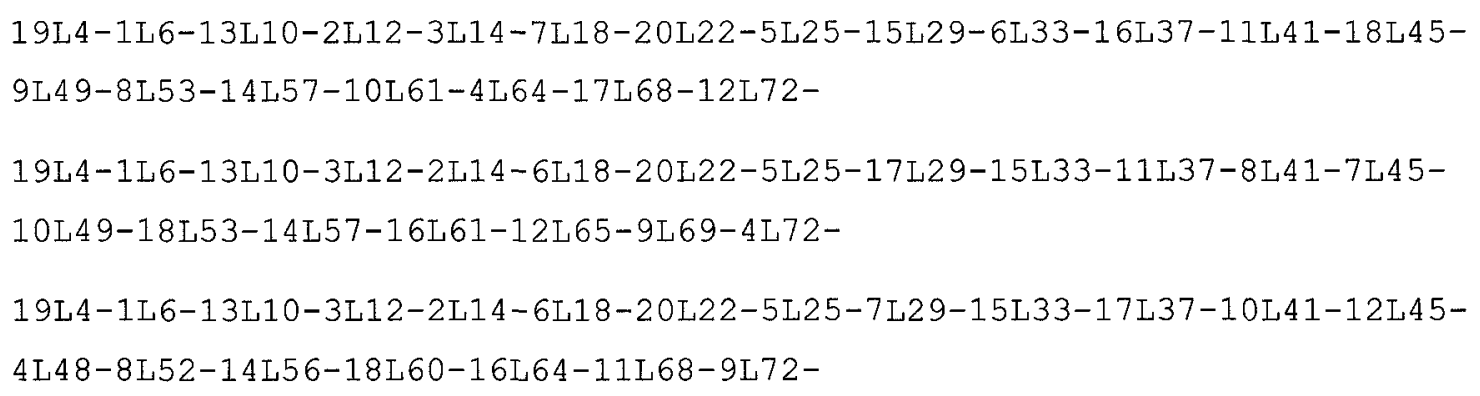

These sequences have the same first three genes: test cases TC19, TC1, and TC13. The fourth and fifth genes, though different, are equivalent genes in terms of additional cost and coverage. For the sixth gene, the GA had the choice between two equivalents (in terms of additional cost and coverage), specifically test cases 6 and 7. The seventh and eighth genes are the same: test cases 20 and 5. At that stage of the sequence, test cases 7 , 15, and 17 are equivalent (cost and coverage). This also applies to test cases 6 and 15 for the tenth gene. At that stage, any permutation of the remaining test cases leads to equivalent test sequences (in terms of coverage) since we have already reached $100 \%$ coverage.

The above analysis shows that the GA has many equivalent candidate values for many genes to select from. Since our current implementation of SPEA2 (in fact the default one) only distinguish the solutions by the phenotype (i.e., the fitness function values), and not the genotype (i.e., the contents of chromosomes), the generated test case sequences (like the three discussed previously) look equivalent to SPEA2 and therefore the selection of one (equivalent) test case over another for a gene becomes random. The non-dominated solutions in the initial generation produce offspring inheriting their gene characteristics. The SPEA2 does not tend to keep solutions showing up with different equivalent genes consciously, so we often observe that the solutions in the SPEA2 archive set only have one or two possible test cases (alleles) for a gene even if there are more (equivalent) candidate test cases (alleles) for that gene. Once the data flow coverage rate reaches 
$100 \%$ from one gene, all successive genes are arranged in random order because all of them are equivalent (same phenotype). Thus in the above examples, we observe that nearer the end of the test case sequences, permutations of test cases look random.

From this case study, we can conclude that data flow information derived at the model level can help identify the case execution sequences. However, data flow analysis at the model level is not as complete as data flow analysis at the code level: models are abstractions of code. For this reason, covering more data flow at the model level does not necessarily lead to killing more mutants. The relation is not necessarily monotonic, as one would likely observe if a data flow analysis at the source code level were used. Indeed, we observed that some complex behavior (i.e., complex algorithms implemented in the code), and therefore complex data flow at the code level does not necessarily correspond to complex data flow at the model level: e.g., the accelerate method (code level) has a complex control and data flow whereas the accelerate operation (model level) is not involved in many data flow elements at the model level. The completeness and precision of model-based data flow analysis appear to be a limiting factor of our approach. We believe that it may not be always practical (or even feasible) to use a model-based data flow analysis as precise as a code-based data flow analysis and we therefore believe that model-based data flow coverage should not be the only criterion when ordering test cases. Some characteristics of the system, such as the (expected) method implementation complexity, or the importance of specific behaviors from an analysis point of view, should be accounted for as well. This will be addressed in future work.

We also observed that GA-evolved test case sequences detect more mutants at the beginning of generated test case sequences than random test case sequences, but not necessarily more mutants later in the sequence. The GA-evolved sequences significantly reduce the mutant detection variance. When GA-evolved sequences are not better than random sequences in terms of mutant detection score, their average (mutant detection score) values are very close to the ones of random sequences but with much less variance. There are two reasons for this result: first, our test cases have many transitions in common and therefore some useful (in terms of mutant detection) test cases are pushed 
towards the end of the sequence by the GA because they are considered too similar to others; second, towards higher cost values, GA-evolved test sequences and random sequences are closer and closer to the full test suite and therefore reach similar mutant detection rates. As well, based on this case study, we observe that GA-evolved sequences based on all-definitions usually outperform the GA-evolved sequences based on all-DU pairs in terms of mutant detection (but not necessarily for highest values of mutation score). We believe this is due to the fact that the test suite covers a larger proportion of definitions than definition-use pairs in the model, as already observed in [5]. We believe this is also due to MuJava. Based on the analysis to the mutants, we find that MuJava tends to seed a large proportion of mutants to defining statements. For instance, for a statement $x=y+z$, more than ten mutants will be seeded into this statement because of the characteristic of MuJava. All these mutants are easily detected if we then reach any use of $\mathrm{x}$. In such a situation, the sequence derived based on all-definitions tends to detect mutants earlier since they cover all definitions earlier.

Also, it is important to remember that the test cases we used and order in a sequence are adequate for the round trip path criterion, which is in essence a control flow criterion (using the criterion a test suite exercises sequences of executions, i.e., control flow). We only use data flow coverage information to rank the test cases. The test suite we used does not cover all the data flow one can identify in the model, as initially observed in a previous work [5].

Last, random curves (mean, minimum and maximum) show mutant detection at each cost value, and suggest that a random sequence could be anywhere in the range between minimum and maximum. Also, this range is quite large and the GA-evolved sequences' average (minimum and maximum) is in the upper part of the range. So a random sequence could, in theory, be slightly better than a GA-evolved sequence at the beginning of the sequence and then worse for the rest of the sequence. Or, a random sequence could, in theory, be always better than a GA-evolved sequence. In other words, given that the range for GA-evolved sequences is very small, we are pretty sure that the average curve is quite close to represent actual sequences. On the other hand, since the variance of random sequences is large, we cannot be sure that the curves for random sequences 
(average, minimum and maximum) do represent actual sequences. By looking at a few random sequences, we did not find a random sequence that is systemnatically (i.e., for each cost value) better than the GA-evolved sequences.

Based on the study of the mutant rate, we find that the mutant distribution significantly affects the mutant rates. MuJava exhaustively seeded mutants in the source code without bias. Its chracteristics cause mutants to be distributed in an unbalance way in such a way that more mutants are seeded in the methods involving more computations. Because of such a set of unbalance mutants, it is sometimes sufficient for a test case to contain a specific transition to kill a large number of mutants: we identified the set of such test cases and called it the powerful set (it kills a large proportion of mutants). As a result, the mutant rates of random sequences are quite close to GA-evolved sequences. We confirmed this by trimming the set of mutants and obtaining a more balanced distribution of mutants per methods. Since mutants in all three case studies are generated by MuJava, these conclusions also apply to the other two case studies.

\subsection{Ordered Set Case Study—Results}

The state machine diagram and all operation contracts for the Ordered Set system are presented in Appendices E.2 and F.2, respectively. Similarly to the Cruise Control system, we present the average data flow coverage rate and the average mutant detection rate at each test cost, as well as their maximum and minimum values at each test cost. "mean_TCS" refers to the average rate (data flow coverage rate or mutant rate) of GAevolved sequences at each test cost. "min_TCS" and "max_TCS" refer to the minimum and maximum values of data flow coverage rate/mutant rate at each test cost. These are compared with the mean, minimum and maximum at each test cost from randomly ordered test case sequences, refered to as "mean_random", "min_random" and"max_random", respectively. We use minimum and maximum values to show the variance between the test case sequences. 


\subsubsection{Ordered Set: Optimization Scheme 1-Specifying a Maximum Test Cost}

In the initial runs, we specify a high test cost $T$ (907, i.e., $99 \%$ of total cost), to make sure that all solutions are able to reach $100 \%$ data flow coverage at $T$. Our GA application shortens $T$ to $T^{\prime}=90$ (around $10 \%$ of total cost) for all-definitions and $T^{\prime}=659$ (around $70 \%$ of total cost) for all-DU pairs, respectively. We then try two GA runs with test cost $T=46$ and $T=182$ ( $5 \%$ and $20 \%$ of total cost) for all-definitions, and two other GA runs with test cost $T=550$ and $T=732(60 \%$ and $80 \%$ of total cost) for all-DU pairs. For values larger than $T^{\prime}$, our GA application always shortens them to values around $T^{\prime}( \pm 3 \%$ of $T$ '). This variation, that we did not observe for the Cruise Control case study is due to the larger size of the Ordered Set case study (larger number of test cases, data flow elements as reported in Table 5), and therefore on the much larger search space. We therefore do not show the results with larger values than $T^{\prime}$ but only show the results with the smaller values than $T^{\prime}$.

As opposed to the solutions of the cruise control system case study, where the GAevolved solutions have exactly the same objective values, the non-dominated GAevolved solutions have different objective values (different in phenotype). As well they are slightly different in genotype.

In Table 14, we show the boundary values of the trade-off frontier of two objectives in terms of objective values, and all trade-off points in graphs. The points indicate the solutions' objective values. For instance, there are three trade-off points for the optimization results of scheme 1 , test cost 46 for all definitions. They correspond to three sets of non-dominated solutions and all solutions within one set are the exactly same in phenotype. One set has objective values (sumCumulCov $=413.72$, CumulCovCost $=91.4 \%$, one set has objective values sumCumulCov $=413.97$, CumulCovCost $=88.2 \%$ and the remaining set has objective values of \{sumCumulCov $=413.76$, CumulCovCost $=90.1 \%$ \}. The three sets of objective values form the trade-off frontier. One set attains the best cumulative coverage rate, one attains the best coverage rate at the end of the user constraint and the other one provides a compromise. The solutions within one set have slightly different test case sequences 
(genotype). Since our non-dominated solutions form several sets of solutions and each set of solutions have the exactly same objective values, it results that the solutions are not evenly distributed in the trade-off frontier. The GA may be able to improve a little bit with additional generations in hope of approximating to the Pareto frontier. Future work will look at other algorithms to increase diversity.

\begin{tabular}{|c|c|c|c|c|c|c|c|}
\hline \multicolumn{2}{|c|}{$\begin{array}{l}\text { All definitons } \\
\text { Test Cost }=907\end{array}$} & \multicolumn{2}{|c|}{$\begin{array}{l}\text { All definitons } \\
\text { Test Cost }=46\end{array}$} & \multicolumn{2}{|c|}{$\begin{array}{l}\text { All DU pairs } \\
\text { Test Cost }=907\end{array}$} & \multicolumn{2}{|c|}{$\begin{array}{l}\text { All DU pairs } \\
\text { Test Cost }=\mathbf{5 5 0}\end{array}$} \\
\hline $\begin{array}{l}\text { SumCumul } \\
\text { Cov }\end{array}$ & $\begin{array}{l}\text { CumulCov } \\
\text { Cost }\end{array}$ & $\begin{array}{l}\text { SumCum } \\
\text { ulCov }\end{array}$ & $\begin{array}{l}\text { CumulCov } \\
\text { Cost }\end{array}$ & $\begin{array}{l}\text { SumCumulC } \\
\text { ov }\end{array}$ & $\begin{array}{l}\text { CumulCo } \\
v \text { Cost }\end{array}$ & $\begin{array}{l}\text { SumCumul } \\
\text { Cov }\end{array}$ & \\
\hline 2416.77 & $100 \%$ & 413.72 & $91.4 \%$ & 137786.77 & $100 \%$ & 95779.63 & $99.2 \%$ \\
\hline 2421.39 & $98 \%$ & 413.97 & $88.2 \%$ & 137799.75 & $99.8 \%$ & 95786.82 & $98.5 \%$ \\
\hline \multicolumn{2}{|c|}{ 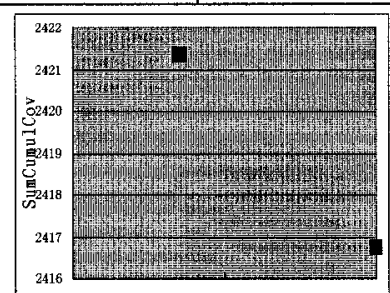 } & \multicolumn{2}{|c|}{ 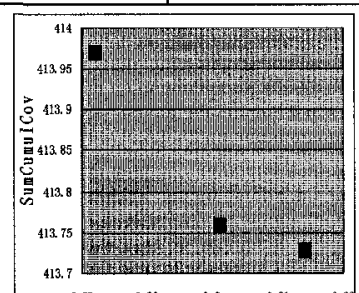 } & \multicolumn{2}{|c|}{ 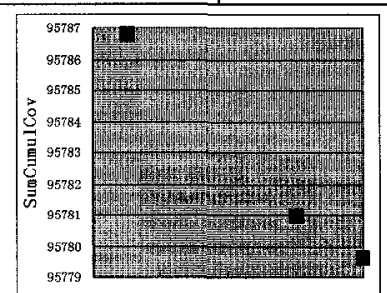 } & \multicolumn{2}{|c|}{ 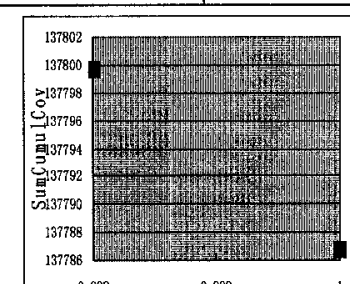 } \\
\hline
\end{tabular}

Table 14: the trade-off objective values of the GA-evolved solutions

In all remaining schemes for this case study, as well as for the VCR case study, we make similar observations regarding trade-off values. We however do not show them since the observations and conclusions are the same.

In Table 15, we provide the results of the GA-evolved sequences and the random sequences in comparison with data flow coverage rate and mutant detection rate of the above four GA runs. Note that when reporting on results that have large test costs for the $\mathrm{x}$-axis, we use a logarithmic scale to facilitate observations. 


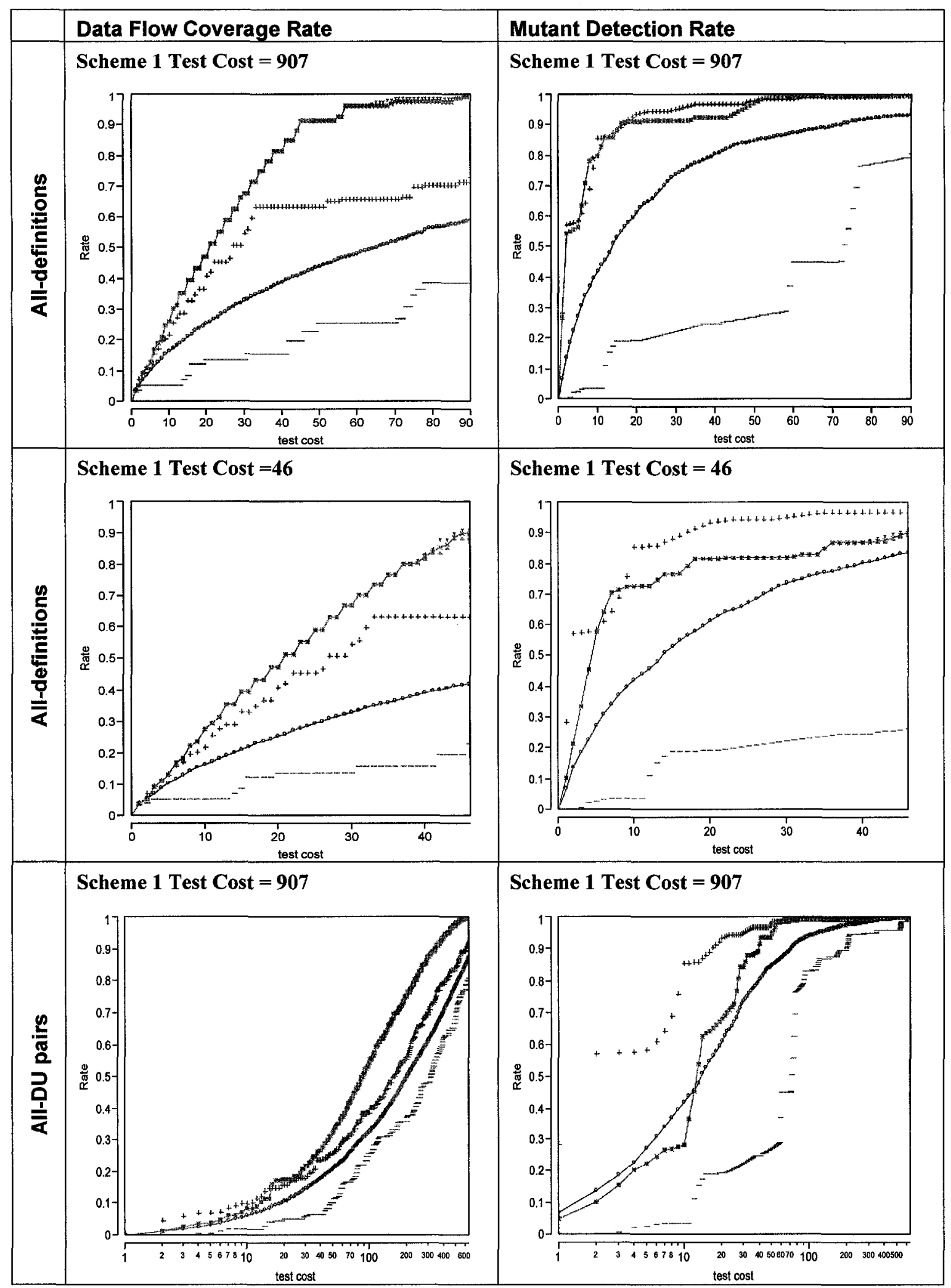




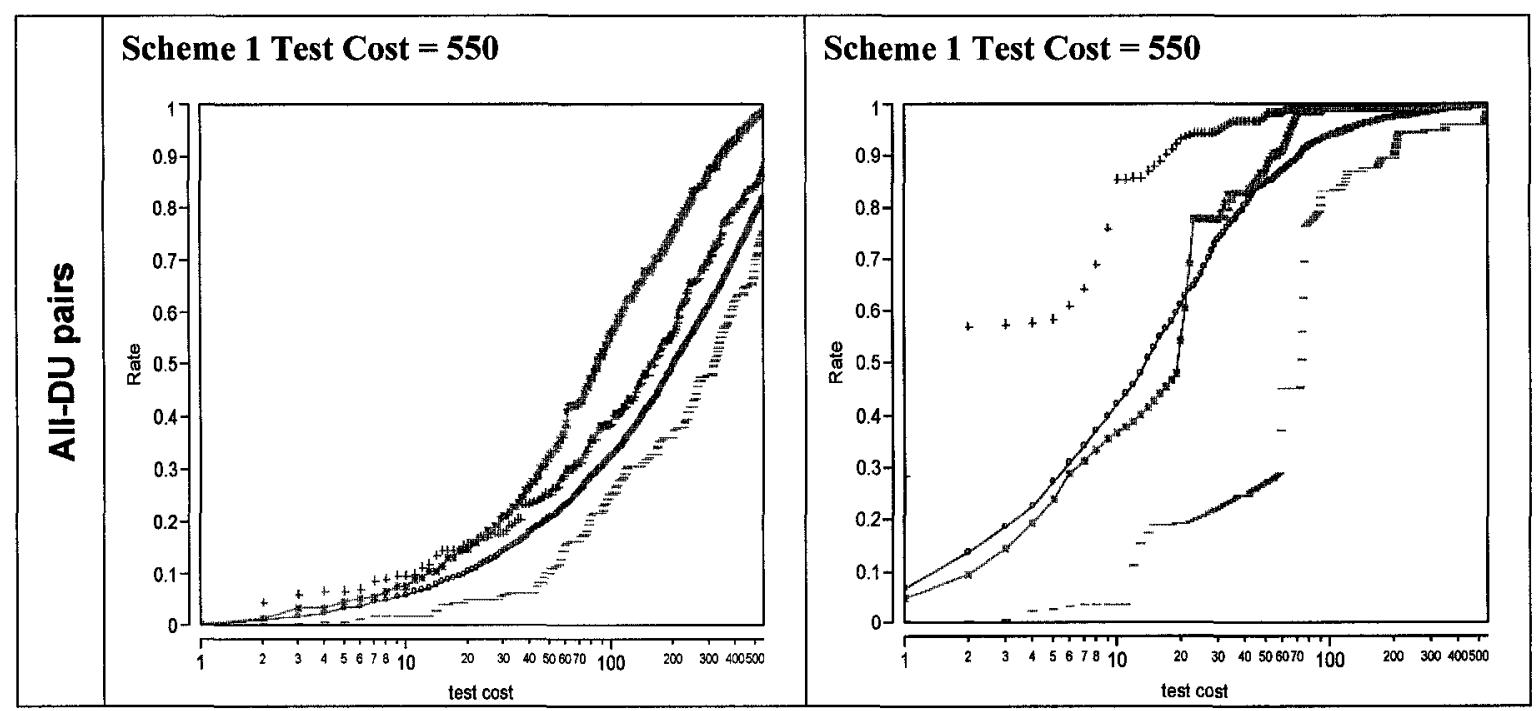

Table 15: Data flow coverage and mutant detection rates of GA-evolved and random sequences (Ordered Set—Scheme 1)

We discuss next coverage rates and mutant rates in two separate sections (Sections 6.3.1.1 and 6.3.1.2). Then we discuss specific characteristics of our search problem that can explain some of the results (Section 6.3.1.3).

\subsubsection{Discussion of coverage rates}

From Table 15, similarly to the previous case study, (1) the GA-evolved sequences increase their data flow coverage rates quickly, which is the intended behavior, (2) the GA-evolved sequences have very small variances, as opposed to random sequences. Using definition coverage makes a clear difference with random. Using a reduced test cost does not change the conclusions.

We notice that the data flow coverage rates of GA-evolved sequences, based on all-DU pairs, are not better than the ones of random sequences during the first 15 test cost values. They are worse than the best random sequence at the first 15 test costs, and close to the average random curve. To explain this observation, recall the objective function for the cumulative coverage rate: $f_{1}(i):$ SumCumulCov $=\sum_{j=1}^{j=T}(T-j+1)^{*} c(i, j)$. If the value of $c(i$, $j$ ), the cumulative coverage rate at test cost $j$ for chromosome $i$, is small and gene $j$ appears early in the sequence $(j$ is small), it does not contribute much though it has a 
large $(T-j+1)$ factor value, and therefore the coverage curve grows slowly and is similar to the average random. This happens in particular if the total amount of data flow elements to cover (here DU-pairs) is very large. If test cases are not extremely long (remember how the transition tree is built), their cumulative coverage rates are all small. This is the case for the OrderedSet case study: the model shows 619 DU-pairs (Table 5), and each of the 165 test cases, with a maximum of 18 transitions (Appendix E) therefore covers a small portion of those data flow elements. Since the GA optimized according to DU-pairs coverage, it then takes a few number of test cases (test costs) to see differences with random sequences. This happens more often with DU pairs than definitions because there are always many more DU-pairs than definitions to cover. We will discuss another possible reason later, namely deceptive attractor.

\subsubsection{Discussion of mutant rates}

Using a large value of allowable cost (i.e., $\mathrm{T}=907$ ), it appears again that the all-definitions criterion performs better (mutant rates) than all-DU pairs: the all-definitions curve is consistently above the random average whereas this is not the case for all-DU pairs (especially early during the sequence). Additionally, both curves reach a mutant score close to $100 \%$ around the same cost value, specifically around $50-60$.

The GA-evolved sequences, based on definitions, detect $80 \%$ mutants at (approx.) cost values 10 and 20 , under different GA runs with different initial cost inputs, and then enter a plateau (though the coverage continues to increase). The random sequences detect $80 \%$ mutants (on average) around cost value 40 .

The plateau is a consequence of (i) the way the test suite, i.e., the transition tree is built, (ii) the way data flow information is identified from the tree, and (iii) the seeding of mutants in the code. Recall (Section 3.1.3.1 and [22]) that when building the transition tree, if a transition has a guard condition that is a disjunction, a branch is added to the tree for each truth value combination that is sufficient to make the guard true. Each added branch in the tree will therefore contain uses of the same model variables: recall Section 3.2). In the result solutions from these GA runs for all-definitions, after test cost value 20 , we observe that all states in the state machine diagram are covered (Empty, PF, Filled, 
Overflow), that each triggering event is covered (e.g., add, remove). By cost value 10 (or 20 ), however, only a subset of the uses has been covered. Since, similarly to the previous case study, it is sufficient to exercise once some methods to kill a large propotion of mutants, we therefore reach a mutant score slightly above $80 \%$ by cost value 10 (or 20 ). The plateau corresponds to the coverage of additional uses due to branch exercising additional combinations of truth value to make guards true. Since the corresponding methods have already been covered, this does not increase much mutant score: thus the plateau. Another reason is the fact that mutants are not seeded in a balanced way, resulting in some test cases killing similar sets of mutants. This was not observed in the previous case study as the state machine did not contain any guard condition.

The curve reaches the plateau faster with initial cost value 907 than with initial cost value 46: there is a sharp increase in mutant score for the first two cost values - half of the mutants are killed at cost value two. We found that the first test case in the sequence (gene 1) - let us label it test case 107 - is quite effective: Test case 107 kills around 54.4\% of the mutants by covering eight definitions and seven du-pairs with cost 2 . This test case exercises the unions of two sets to generate one new ordered set which size is just below the maximum acceptable size and then successfully removes one item from the new set. By looking at the source code, we discover that method union() uses (directly or indirectly) all the most complex methods of the class. Since many mutants are killed by simply executing the methods where they are seeded, and because a large proportion of the mutants are seeded in the methods (in)directly called by union (), that test case becomes a quite effective test case.

We notice the random sequences do very well in terms of mutant rates. Based on our past experience with the cruise control case study, we looked at the mutants. We find that $42 \%$ of the mutants (240 out of 575) are seeded into method union () and around $45 \%$ of the test cases ( 75 out of 165) invoke this method. 52 of those 75 test cases perform a union of two sets as similar as test case 107 discussed previously. These form a powerful set of test cases, a situation which we also encountered in the previous case study (section 6.2.1). These test cases therefore tend to kill most of the easy to kill mutants of these 240 mutants. Their mutant rates are between $40 \%$ and $55 \%$. The fact that random sequences 
are close to GA-evolved sequences is therefore a result of test cases that exercise a lot of the functionalities at once and kill the seeding of mutants (as discussed for the previous case study).

Based on all-DU pairs, the GA-evolved sequences detect fewer mutants than the random sequences during the first 10 or 20 test cost values, under different GA runs with the different initial cost input ( 907 or 550). They then show a sharp increase to quickly detect around $80 \%$ mutants at a cost value around 20 . We notice that in the two GA runs (cost=907 and cost=550), GA-evolved sequences start with the test cases that are not very effective at killing mutants until around test cost 10/20. First we observe that MuJava seeded mutants that are easy to kill by simply hitting uses of variables (in the source code, and as a consequence in the model). Covering du-pairs rather than definitions does not help improve the results, on the contrary: test cases that cover the most du-pairs do not appear to cover the most definitions (e.g., a test case can cover two du-pairs but the two du-pairs involve the same definition). Second, random sequences perform well for the reasons already explained above: 52 test cases out of 165 (i.e., 31.5\%) are very effective at killing mutants.

\subsubsection{Deception during GA search}

Based on the analysis of the solutions from the different GA runs, we observe two things we did not notice in the first case study.

First, the solutions from one GA run (i.e., the non-dominated solutions in the archive) are very similar in genotype (subsequences of test cases up to a specific cost value: little differences in sub-sequences can be noticed) but the solutions from different GA runs are quite different in genotype (subsequences of test cases up to a specific cost value: subsequences with little things in common can be noticed). For instance, based on all-DU pairs, all solutions from the GA run with input $T=907$ start with subsequence ${ }^{11}$ : 19L428L 7-57L10-155L14-125L25-133L28-... But almost all solutions from the GA run with

\footnotetext{
${ }^{11}$ The format in the test case sequences shows test case numbers and incremental costs: e.g., 107L2$72 \mathrm{~L} 5$ means the first test case is TC107 ending at test cost 2 , and the second test case is TC72 ending at test cost 5 .
} 
input $T=550$ start with subsequence 35L6-72L9-19L13-134L19-55L21-84L23-39L25$54 \mathrm{~L} 27-\ldots$.

The second observation is that, based on an analysis of data flow information for all definitions, we know - we used a greedy search method - that the best choice for the first gene is test case 99 which covers the maximum number (9) of definitions with cost 2, and that the second best choice is one of a set of 11 other test cases in which each test case covers the second maximum (8) number of definitions with cost 2 . However, the GA does not often present solutions with the best choice first. Instead, it usually presents solutions with the second best choice first, even occasionally the third best choice first.

To explain the first observation, let us first remind that the fitness landscape can have numerous, possibly high peaks. These are called multimodal problems, each peak being referenced to as a mode. In multi-modal problems, "simple ${ }^{12}$ GAs usually converge to a single peak, even though multiple peaks of equal quality may exist" [53] because they do not deliberately control the competition between solutions, i.e., they do not use parts of the chromosomes (genotype) to keep solutions that show unique, good characteristics (phenotype) and discard solutions that show similar (or equal) characteristics. In other words, they do not keep solutions that show unique, good characteristics (phenotype) and discard solutions that show similar (or equal) characteristics. A GA applying the concept of niche and sharing to the fitness space ${ }^{13}$ can better exploit the search space and have a higher probability to find alternative peaks. But it does not always succeed because of many other factors such as the niche diameter, the sharing method, the size of the search space, the number of modes, the magnitude of the differences between peak fitness values, the size of the population $[53,54]$. The GA algorithm we used in our application (SPEA2) only applies the concept of niche and sharing to the phenotype (i.e., fitness values). Without applying those concepts to the genotype in addition to phenotype, all solutions from the same GA run look similar to each other in genotype but have different

\footnotetext{
12 These are GAs that do not deliberately control the competition between solutions. Controlling competition means kepping solutions that show unique characteristics and discarding solutions that show similar (or equal) characteristics.

${ }^{13}$ A niche is a specific sub-domain of the solution space to which some individuals belong; Sharing is the method used to identify similarities between solutions and determine niches [6].
} 
objective values (phenotype). This is one reason for the first observation discussed previously (solutions of one GA run have similar genes, i.e., similar genotype). Another reason is introduced below when talking about the second observation.

To explain the second observation (solutions from different GA runs are very different in genotype), we have to introduce the notions of deception and deceptive attractors in ordering genetic algorithms. Deception is a phenomenon where a GA is misled to a local optimum rather than a global optimum. A deceptive attractor is a specific order for some particular genes in the sequence, i.e., a sub-sequence in the chromosome, that will lead to a local optimum rather than a global optimum. Kargupta, Deb and Goldberg [55] reveal how ordering problems can be deceptive - i.e., how ordering multimodal problems are misleading in genetic algorithms. They designed two deceptive ordering problems in which the best sub-sequence contains a deceptive attractor that is maximally distant from the global optimum. They tried to reveal if an adequate crossover operator could get rid of deceptive attractors. They therefore studied different crossover operators for their problems: the search space is $\left.(4 !)^{8} \approx 1.1 * 10^{11}\right)$. Using a population size of 2500 and 100 generations, they observed that no single crossover operator is adequate to find the global optimal solution in both ordering problems. Whitley and Yoo [54] pointed out that deceptiveness of ordering problems depends in part on the magnitude of the differences between global optimum fitness values and local optima fitness values. In our problem, unfortunately, the search space is too large $\left(165 ! \approx 5.4 * 10^{295}\right)$ to investigate whether the GA encountered (and was deceived) by a deceptive attractor: e.g., we do not know and cannot identify the global optimum. We can argue though that the chances of deceptive attractors in our problem are high. Indeed, we know that test case 99 is the best choice for gene 1 which will lead to many peaks in the fitness space since there are then many equivalent test cases for gene 2 . On the other hand, 11 other test cases are the best second choice for gene 1 which will also lead to many peaks in the fitness space. Therefore these 11 test cases are deceptive attractors if test case 99 leads to the global optimum. Or, if all these 11 test cases lead to global optimums, then it is not surprising that for each GA run different solutions in genotype are presented: each time one of the 11 can be selected. Alternatively, if only one of those 11 test cases leads to the global optimum, then all the other ten test cases are deceptive attractors. And this discussion only accounts for the first 
genes. We therefore believe that in our problem our GA can easily be trapped in a deceptive attractor, which explains why different GA runs may result in quite different sequences in genotype and why the first gene(s) (test case/cases) is often not the best.

\subsubsection{Ordered Set: Optimization Scheme 2-Specifying a Cumulative Coverage Rate}

In this optimization scheme, we specify two user expected cumulative coverage rates (SC) of $80 \%$ and $90 \%$ for definition and DU pair coverage. The comparison result is presented in Table 16: we use a logarithmic scale on the x-axis. Similarly to the previous case study, and as discussed in 6.1.2.1, it is not surprising to see a non-monotonic increase in data flow coverage rates of random sequences after $T_{\text {shortest }}$, the shortest test cost a set of sequences take to reach $S C$. The GA-evolved sequences take very similar test costs to reach $S C$ and their data flow coverage rates and mutant rates at these test costs are very close, so the non-monotonic increasing values are not so obvious for the GAevolved sequences.

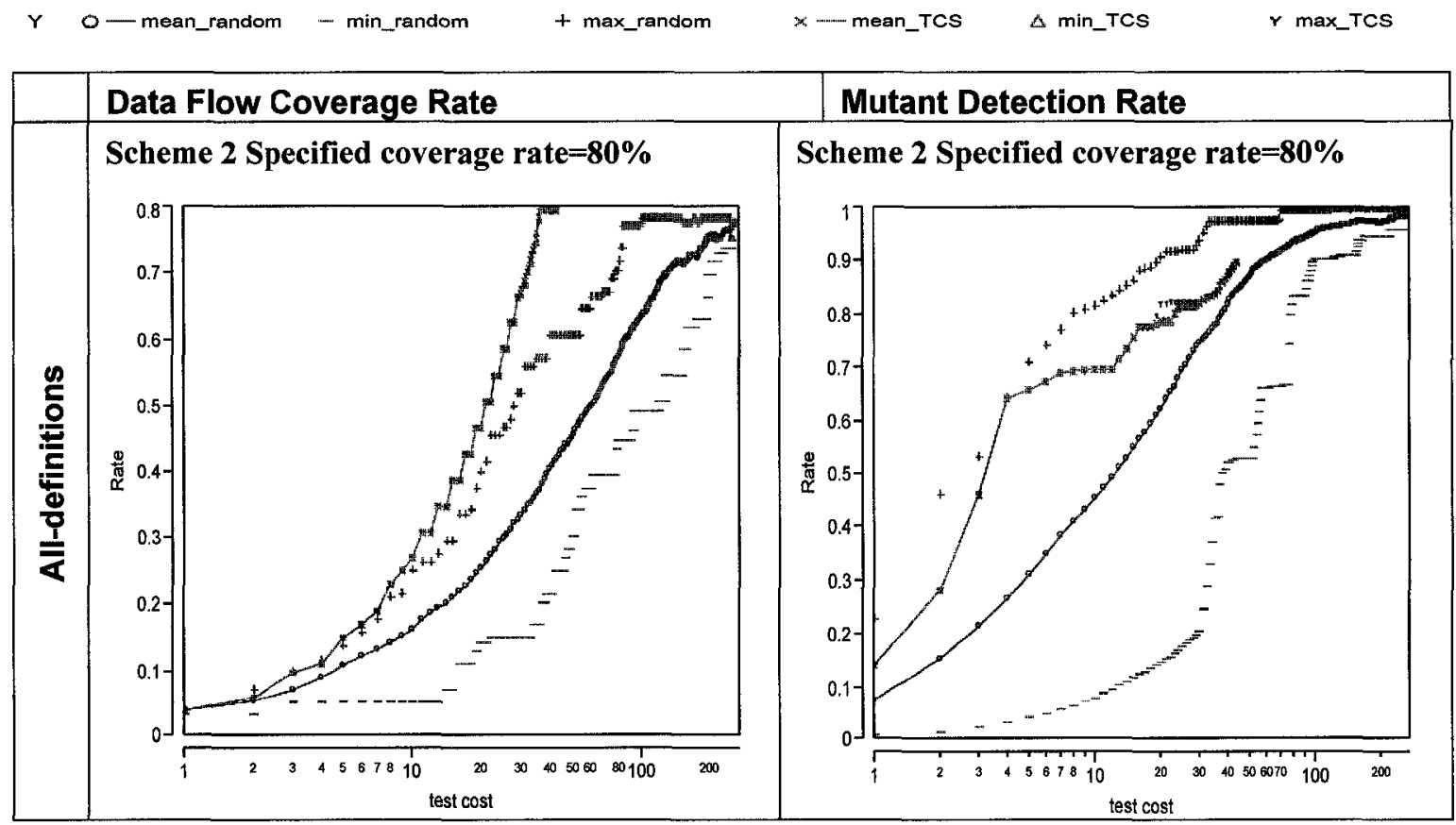




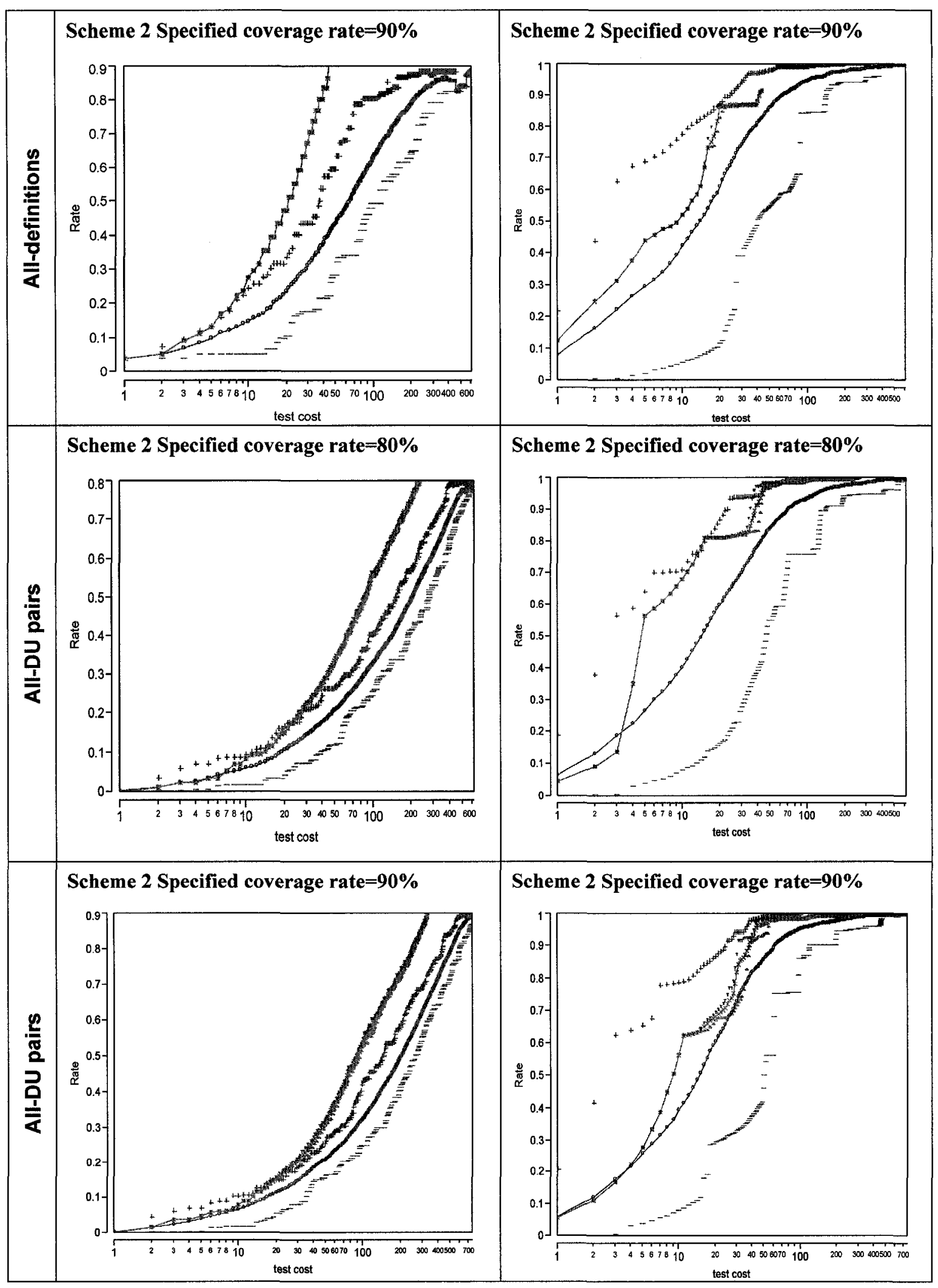

Table 16: Data flow coverage and mutant detection scores of GA-evolved and random sequences (Ordered Set-Scheme 2) 
With a data flow coverage rate of $80 \%$ or $90 \%$, the GA-evolved sequences based on allDU pairs or all-definitions increase their data flow coverage rates quicker than the random sequences, which is the intended behavior. The test costs of achieving a given coverage are significantly different for all-definitions and all-DU pairs: cost of 36 (resp. 44) to achieve 80\% (resp. 90\%) definitions; cost between 224 and 233 (resp. between 323 and 327 ) to achieve $80 \%$ (resp. 90\%) du-pairs. Criteria are cheaper to achieve such high coverage percentages than random sequences (roughly three times more): random sequences take test costs between 100 and 247 (resp. between 156 and 611) to achieve $80 \%$ (resp. 90\%) definition coverage; random sequences take test costs between 441 and 622 (resp. between 597 and 757) to achieve 80\% (resp. 90\%) du-pairs coverage. The GAevolved sequences based on all-definitions detect around $10 \%$ mutants more than the random sequences in general; the GA-evolved sequences based on all-DU pairs detect around 5\% mutants more than the random sequences except during the first 5 cost values. In section 6.3.1, we have mentioned that mutant rates of random sequences are quite good and that DU-pair coverage is not very effective at detecting mutants in our case study: we make similar observations here. We also observe usually there are a sharp increase and a plateau in the mutant rates of the GA-evolved sequences. In section 6.3.1, we already explained reasons for that: 1) the unbalanced mutant distribution causes some test cases to be quite effective; 2) the GA tends to present similar solutions in genotype (the test case sequences contain very similar subset of test cases with similar orders). When a very effective test case is ranked we see a sharp increase and the following test cases usually do not kill many more mutants (though they add to the coverage) - plateau.

Simiarly to the previous analyses, we observe a much larger variance of random sequences curves (coverage and mutant detection) than for GA-evolved sequences. The GA-evolved sequences for $S C=90 \%$ show some variance in mutant rates from cost 10 to 60 because some test cases increase data flow coverage in similar ways but they have different mutant rates.

We also observe that it is more expensive for random sequences than GA-evolved sequences to achieve specific, high mutant rates: using all-definitions, the GA-evolved sequences reach $80 \%$ (resp. 90\%) mutant rate at cost 20 (resp. between 36 and 44 
because of variance in GA solutions); using all-DU pairs, the GA-evolved sequences detect $80 \%$ (resp. $90 \%, 99 \%$ ) mutants at cost 15 to 30 (40 to 60,60 to 200); The random sequences reach $80 \%$ (resp. 90\%, 99\%) mutant score at cost 35 (resp. 60, 300).

\subsubsection{Ordered Set: Optimization Scheme 3-Specifying 100\% Cumulative Coverage Rate and Maximum Available Test Cost}

In this optimization scheme, we do not specify any user expected data. This scheme simply uses $100 \%$ cumulative coverage rate and $100 \%$ cost. The comparison result is given in Table 17: SC is the user specified coverage rate $100 \%$ (i.e., 1) and $\mathrm{T}$ is $100 \%$ test cost 916. Note that we use a logarithmic scale to facilitate observations.

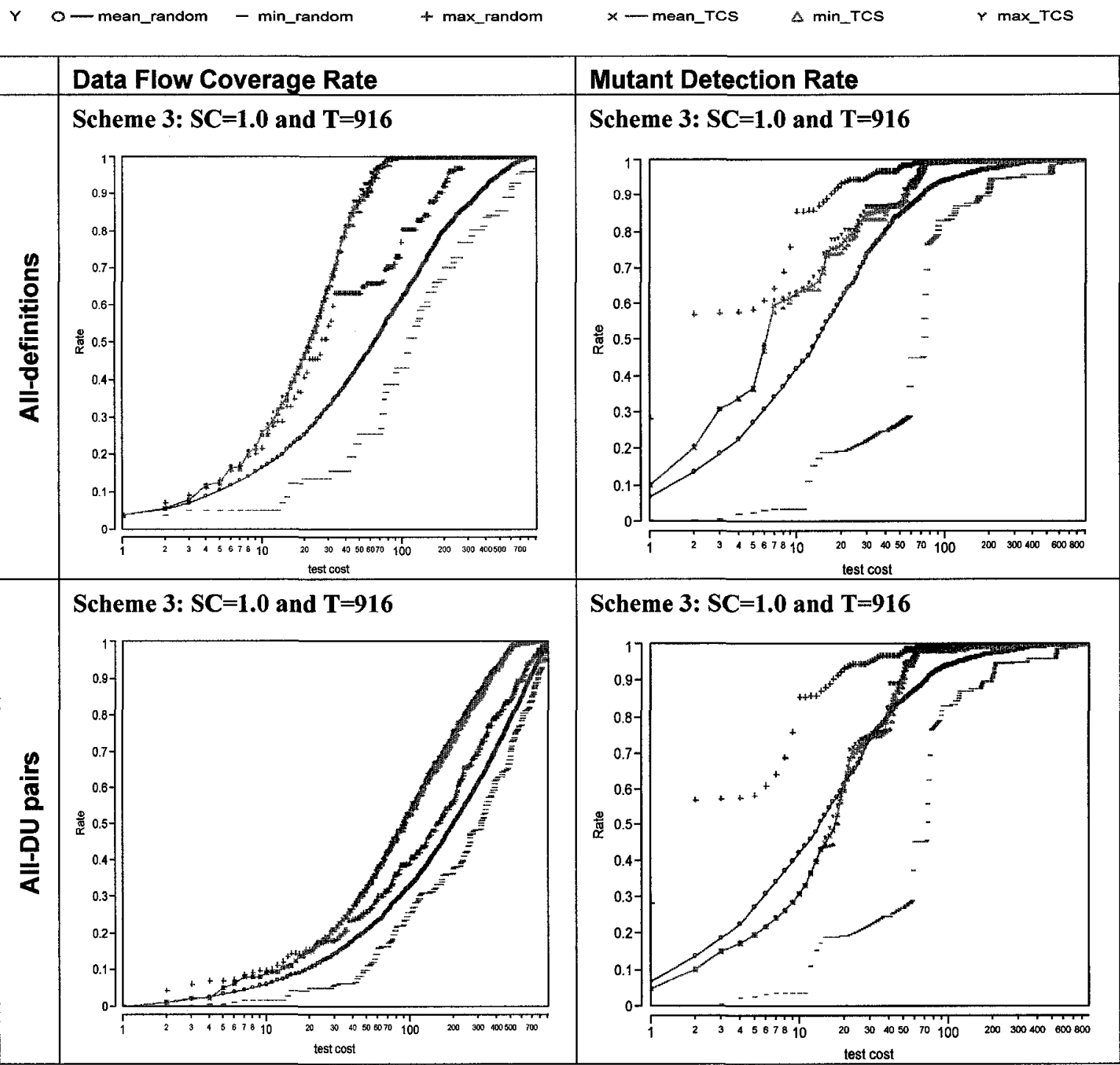




\section{Table 17: Data flow coverage and mutant detection rates of GA-evolved and random sequences (Ordered Set—Scheme 3)}

We observe that the GA-evolved sequences increase their data flow coverage rates quicker than random sequences, with much smaller variances. The GA-evolved sequences cover $100 \%$ definitions at cost 93 and cover $100 \%$ DU pairs at costs between 590 and 601.

The GA-evolved sequences based on all-definitions detect $99.8 \%$ of the mutants when all definitions are covered (i.e., at cost 93). The GA-evolved sequences based on all-DU pairs detect $100 \%$ of the mutants when all DU pairs are covered (i.e., at cost around 600). Table 17 therefore shows that definition coverage is more cost-effective than du-pair coverage: achieving $100 \%$ definition coverage is much cheaper than achieving $100 \%$ dupair coverage for (almost) the same effectiveness. In general, based on either data flow criterion, the GA-evolved sequences detect $95 \%$ of the mutants at test costs 50 to 60 , but the random sequences detect $95 \%$ mutants at test costs 110 to 120 ; the GA-evolved sequences detect $99 \%$ of the mutants at test cost around 100 but the random sequences detect $99 \%$ of the mutants at test cost around 300. Again, we observe the GA-evolved sequences based on all-definitions perform better in mutant rates than the GA-evolved sequences based on all-DU pairs for the first 40 (approx.) test costs. After that, the GAevolved sequences based on different criteria do not show significant differences in mutant rates.

We also observe the GA-evolved sequences for both criteria have a sharp increase and plateaus in the mutant rates and show variances in the mutant rates. We already mentioned the reasons for the sharp increase, the plateaus and the variances in sections 6.3.1 and 6.3.2. The same reasons apply here.

We also notice that the GA-evolved sequences based on all-DU pairs are not better at detecting mutants than the random sequences for the first 20 cost values. We find the GA-evolved sequences usually begin with the following two subsequences: 57L3-50L1187L14-28L17-70L19-131L23-... and 57L3-50L11-87L14-135L20-70L22-131L26.... In both subsequences, the only powerful test case, test case 131 , appears from test cost 20 or 23 , so 
the mutant rates of the GA-evolved sequences increases significantly from test cost 20 and exceeds the random sequences. Then test cases that are not powerful at killing mutants and are arranged after that cause the mutant rate to increase quite slowly until test cases 107 and 84 from the powerful set appear from test costs 40 to 55 , at which point we see a second sharp jump in the mutant rate curve. In considering the large number (52) of powerful test cases, it is not surprising that the random sequences could do better than the GA-evolved sequences for the first 20 cost values.

\subsubsection{Ordered Set: Optimization Scheme 4-Specifying a Maximum Test Cost and a Minimum Cumulative Coverage Rate}

In this optimization scheme, we specify a maximum test cost and a minimum cumulative coverage rate. Since our GA application can heuristically shorten the test cost to a value that satisfies the minimum cumulative coverage rate, we simply specify a relatively large test cost and combine it with the data flow coverage rates $S C$ of $80 \%$ and $90 \%$. So in the initial GA run, we specify test costs 732 and 824 (80\% and $90 \%$ of total cost) for alldefinitions and all-DU pairs, respectively, to make sure almost all solutions can reach the minimum $S C$ at the end of test case sequences. We present the comparison results based on the initial user input data in Table 18. Note that when reporting on results that have a large test cost range (x-axis), we use a logarithmic scale to facilitate observations.

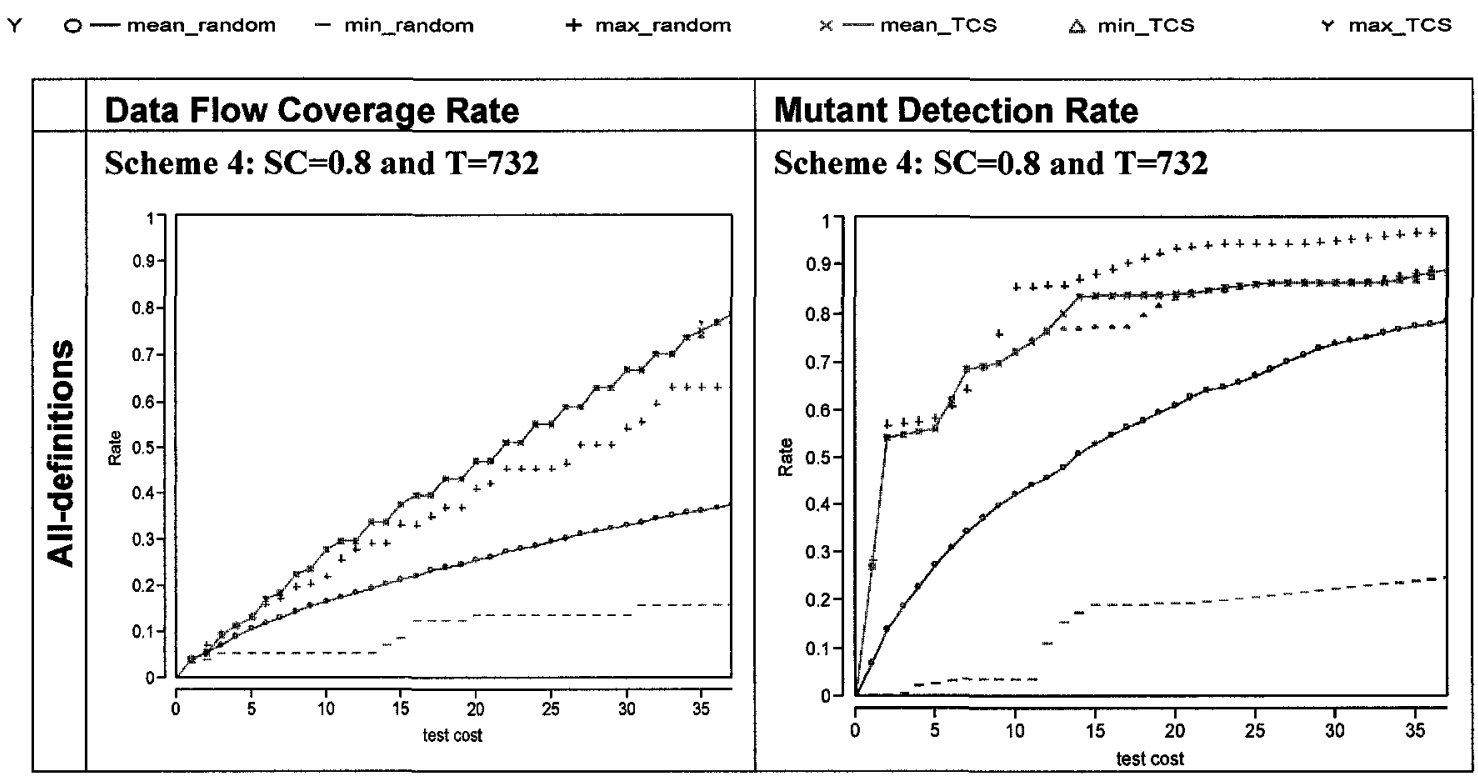




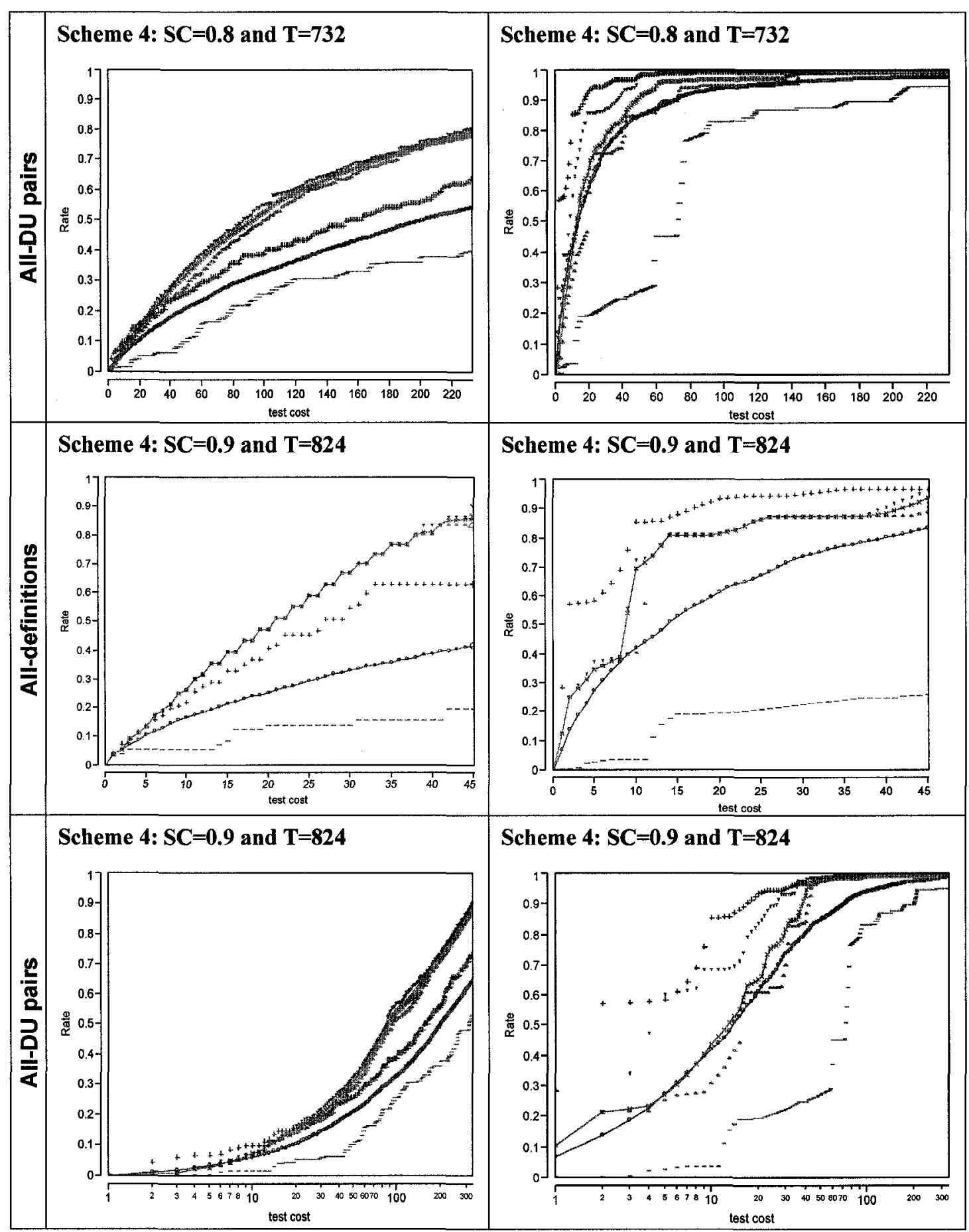

Table 18: Data flow coverage and mutant detection rates of GA-evolved and random sequences (Ordered Set-Scheme 4)

With $80 \%$ and $90 \%$ data flow coverage rates $(S C)$ and $80 \%$ and $90 \%$ of total costs $(T)$, the GA-evolved sequences increase their data flow coverage rates quicker than the random sequences, except for the initial part of the sequence (please refer to the 
explaination in section 6.3.1.1), and reach $S C$ with less test cost than $T$. To cover $80 \%$ and $90 \%$ of all definitions, the GA-evolved sequences need 36 and 44 test costs at least, respectively, so $T^{\prime}$ is set to 37 and 45 respectively. To cover $80 \%$ and $90 \%$ of all DU pairs, the GA-evolved sequences need 226 and 323 test costs at least, respectively, so $T^{\prime}$ is set to 233 and 335 respectively (recall that $T^{\prime}$ is the average test cost where the GAevolved sequences reach $S C$ ). Again, the random sequences show much higher variations than GA-evolved sequences. Similarly to the first case study, it appears that optimization for definition coverage performs better than optimization for du-pair coverage (when comparing curves to the random average). GA-evolved sequences usually have a mutant score $10 \%$ higher than the one of random sequences (on average) from test costs 20 to 100 ; then the mutant rates get closer as the test cost increases because the mutant rate of GA-evolved sequences already reach more than $99 \%$.

We notice some legible variance in the data flow coverage rate of sequences optimized for du-pair coverage with $S C=0.8$ and $T=732$. We find that the solutions presented by the GA have either subsequence 28L3-55L5-40L7-77L11-69L13-155L17-63L21-70L2315L38-133L41-105L46-120L49-42L52-... or subsequence 40L2-87L5-123L12-42L1569L17-70L19-133L22-118L26-28L29-85L31-57L34-61L37-107L39-39L41-19L45113L50-72L53-... Although the two subsequences are not equivalent in terms of data flow coverage, both sequences are preserved by the different objective funtions (here we have four objective functions). We find that the GA, likely by chance, exploites two modes represented by these two sub-sequences.

Since our GA application shortens $T$ to $T^{\prime}$, we use $T^{\prime}$ in the second set of GA runs. A larger value than $T^{\prime}$ is used to verify if our GA application consistently shortens $T$ to $T^{\prime}$ if $T$ is large enough to satisfy $S C$. A smaller value than $T$ ' is also used to verify our GA application will present a new $S C$ ' and tell the user that the cost budget is not enough to satisfy $S C$ but the user could expect $S C^{\prime}$ within $T^{\prime}$. So in the following GA runs, for alldefinitions, we use a new $T$ equal to 27 and 92 for $S C=80 \%$, and 37 and 92 for $S C=90 \%$; for all-DU pairs, we use a new $T$ equal to 183 and 278 for $S C=80 \%, 275$ and 458 for $S C=90 \%$. We observe that when the new $T$ values are larger than $T^{\prime}$, our GA application again shortens them to values around $T^{\prime}$ ( $\pm 3 \%$ of $\left.T^{\prime}\right)$. We therefore do not present those 
results and we only present the comparison results based on the combinations of values smaller than $T^{\prime}$ and two cumulative coverage rates $80 \%$ and $90 \%$ in Table 19.

$\checkmark \quad O-$ mean_random - min_random $\quad+$ max_random $\times$ - mean_TCS $\Delta$ min_TCS

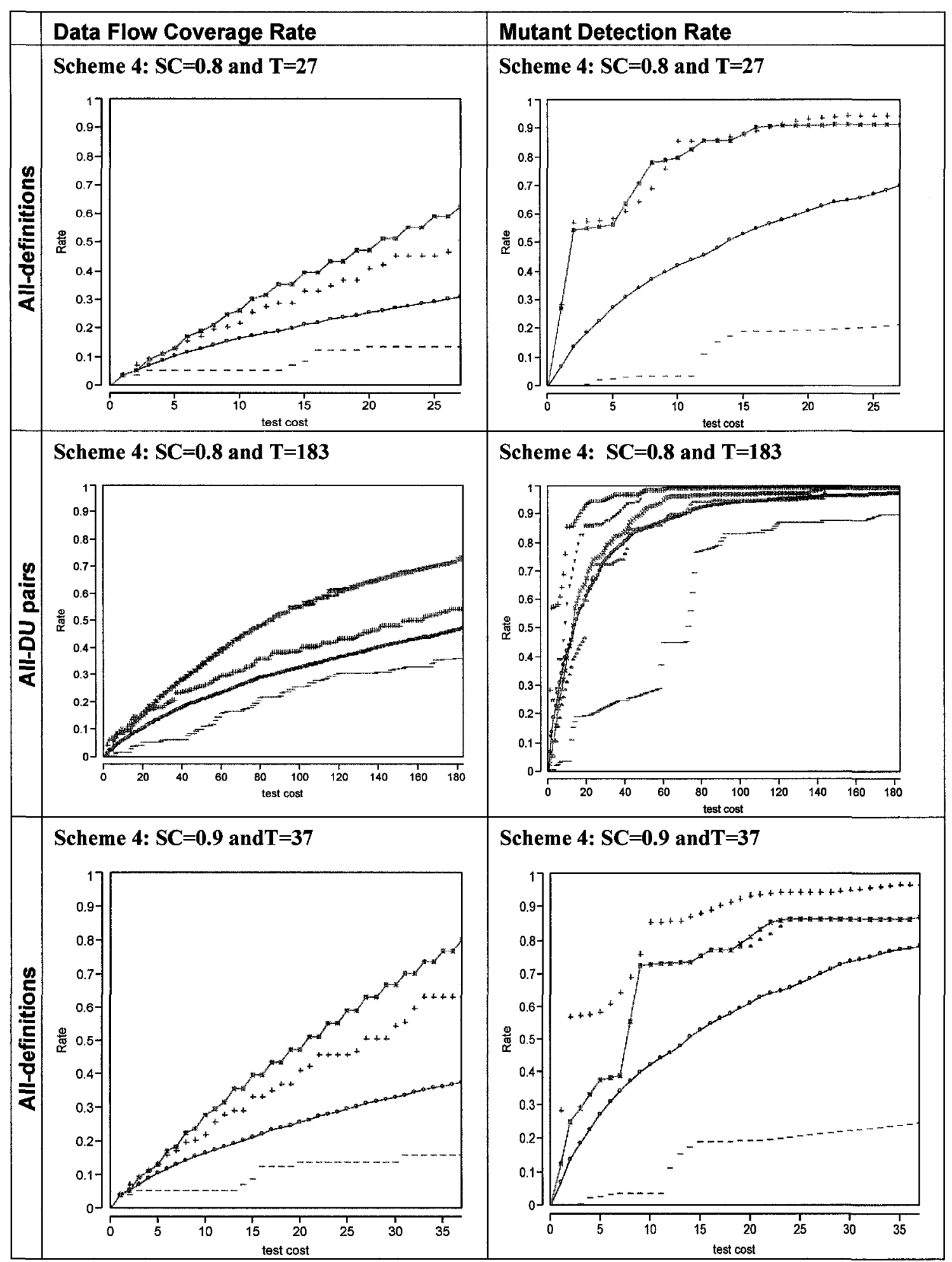




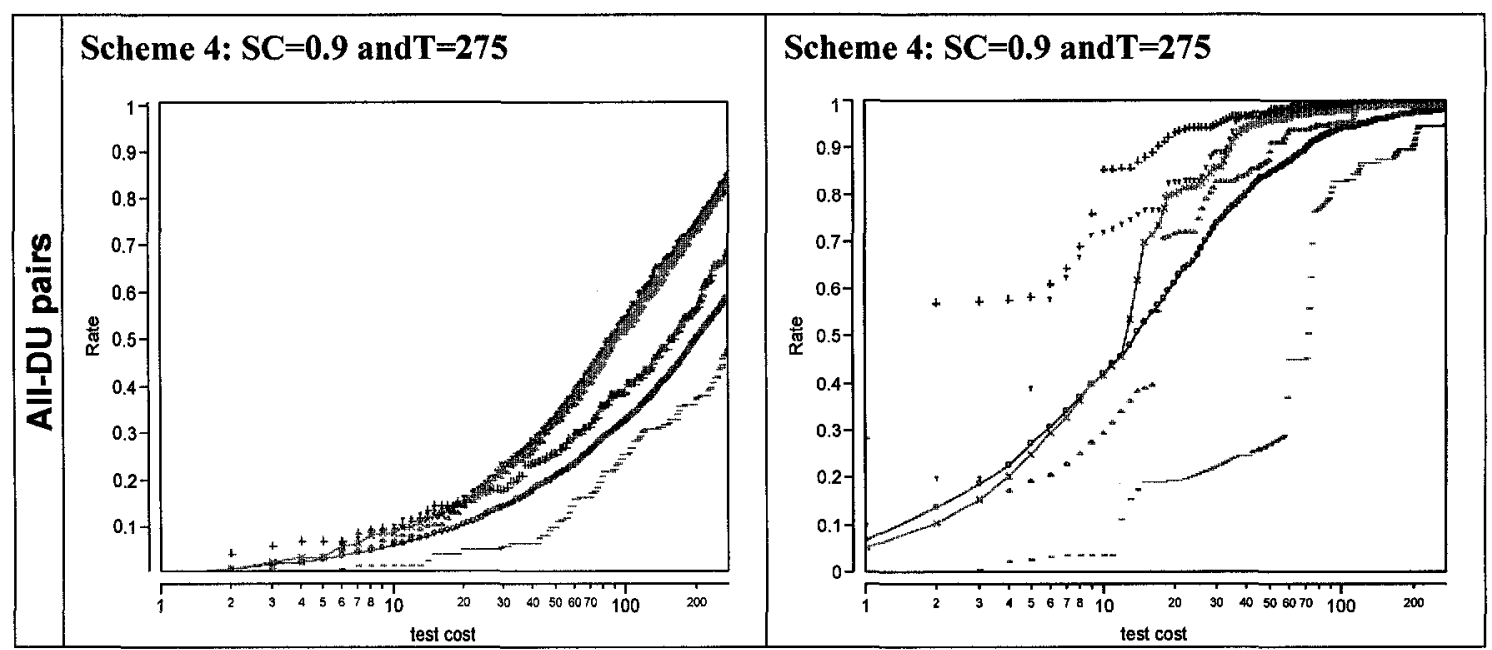

Table 19: Data flow coverage and mutant detection scores of GA-evolved and random sequences with new Ts (Ordered Set-Scheme 4)

At test costs 27 and 37, the GA-evolved sequences (based on definition coverage) cover $63 \%$ and $76 \%$ of all definitions, respectively; at test costs 183 and 275 , the GA-evolved sequences (based on du-pair coverage) cover $74 \%$ and $85 \%$ of all DU pairs, respectively. The GA-evolved sequences have less variance than the random sequences. The GAevolved sequences often detect more mutants for small cost values with less variance than the random sequences.

The sharp increase and the plateaus in mutant score curves have already been discussed and explained in previous sections (such as 6.3.1 and 6.3.2). We do not repeat them again here. We also observe that GA-evolved sequences based on all-DU pairs $(S C=0.9$ and $T=275$ ) show a legible variance in the data flow coverage rate at the beginning part of sequences (up to test case value 20). From the data flow analysis, we identify the same reason as what we mention in section 6.3.3: Non-equivalent subsequences are preserved by different objective functions and the GA may not evolve enough. With different subsequences at the beginning of the evolution, we see some significant variance in the mutant rate since the mutant rates of test cases are very different.

\subsubsection{Ordered Set: Study of the variance between GA runs}

We have noticed moderate variances in the data flow coverage rates as well as mutant rates of GA-evolved sequences. The min and max curves only show the variance within 
one GA run. Because of the (likely) occurrence of deceptive attractors in our ranking problem, we can expect that different GA runs will return different solutions in genotype even though they may have similar objective function values (phenotype). We therefore study the variance between GA runs, and what these (possibly) different solutions look like.

To answer these questions, we run $20 \mathrm{GA}$ trials using evolution scheme 3, based on alldefinitions. The $20 \mathrm{GA}$ runs return 20 sets of quite different test case sequences (genotype) with very similar objective values (phenotype), as expected. We randomly take one GA-evolved sequence from each trial to form a set of GA-evolved sequences. These $20 \mathrm{GA}$-evolved sequences are compared with 20 random sequences. We present our comparison result in Table 20, where we show all data: data flow coverage rate and mutant detection rate at each test cost for each test case sequence, as well as the minimum, maximum and average mutant rates of 20 test case sequences.

From Table 20, we find that all the data flow coverage rate curves of $20 \mathrm{GA}$-evolved sequences increase much quicker than the random sequences and reach $100 \%$ data flow coverage rate at around 100 test costs (instead of an average cost of 800 for random sequences). The variance between the $20 \mathrm{GA}$-evolved sequences is very small. Plus, the 20 random sequences have a great deal of variance in the data flow coverage rates. The 20 GA-evolved sequences cover $100 \%$ of all definitions at the shortest test cost 94 and the longest test cost 104, while the best random sequences cover $100 \%$ of all definitions with the test cost 463 .

The mutant rates of the $20 \mathrm{GA}$-evolved sequences have a $10 \%$ to $15 \%$ advantage over the mutant rates of the 20 random sequences for test costs 3 to 40 on average, and have on average a $5 \%$ to $10 \%$ advantage for test costs 41 to 100 . Moreover, the variance of the mutant rates of the $20 \mathrm{GA}$-evolved sequences is smaller than the variance of the mutant rates of the 20 random sequences. When the GA-evolved sequences cover $100 \%$ of all definitions with test costs 94 to 104 , they detect $99 \%$ of all mutants on average (the minimum value of $95.8 \%$, the maximum value of $99.8 \%$, the median value of $99.8 \%$ ). As a comparison, at test cost 94 , the random sequences detect $93.3 \%$ of all mutants on 
average (the minimum value of $73.9 \%$, the maximum value of $99.3 \%$, and the median value of $94.2 \%$ ); at the test cost 104 , the random sequences detect $94 \%$ of all mutants on average (the minimum value of $84.7 \%$, the maximum value of $99.4 \%$, the median value of $94.4 \%)$.

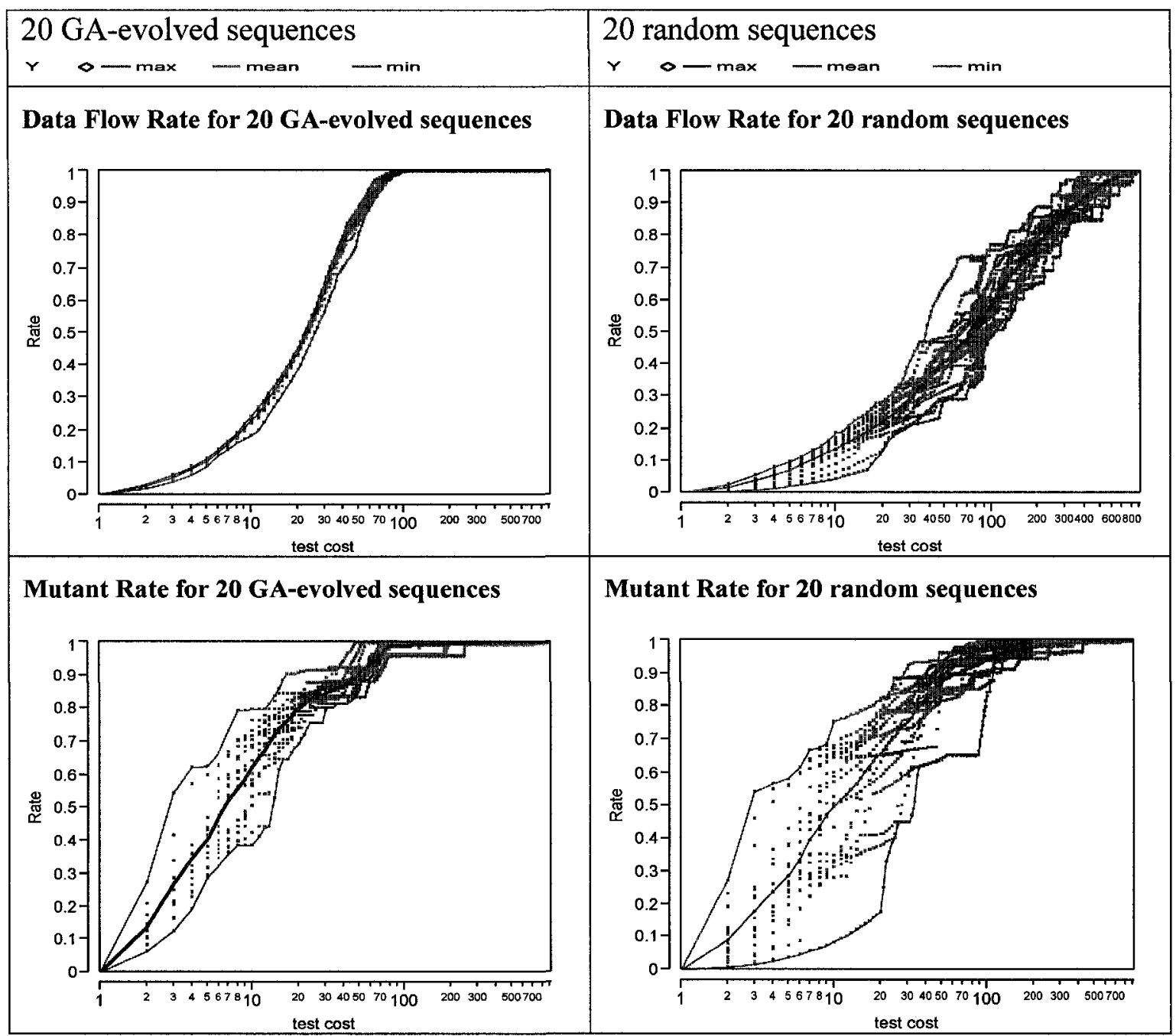

Table 20: Data flow rate and mutant rate in comparison with 20 GA-evolved sequences from 20 trials and 20 random sequences-OrderSet System.

From this study, we conclude that (1) though the deceptive attractors exist, the GAevolved sequences have very small variance in the data flow coverage rates (in phenotype); (2) because the GA-evolved sequences are different in genotype, their mutant results show significant variance. In the comparison with the random sequences, the GA-evolved sequences show smaller variance in data flow coverage rate and mutant rate, as well as the tendency of detecting mutants earlier. 


\subsubsection{Ordered Set: Summary of Results}

In this case study, the GA-evolved sequences increase their data flow coverage rate quicker and earlier than the random sequences. The different data flow criteria take different test costs to reach the specific coverage goals. In the mutant rate comparison, the GA-evolved sequences based on all-definitions usually do better than the ones based on all-DU pairs. Based on all-definitions, the mutant rates of the GA-evolved sequences are usually better than those of the random sequences at the first 100 test costs. Based on all-DU pairs, the mutant rates of the GA-evolved sequences are usually better than, except occasionally close to, those of random sequences for costs 20 to 100 . Then after that, the mutant rates of the GA-evolved sequences based on both criteria are quite close to $100 \%$. The GA-evolved sequences have very small variance in the data flow coverage rate, but their mutant rates do not necessarily have small variance. This indicates that the data flow information in the operation contracts has some difficulties to express the source code complexity exactly. This illustrates the gap between the analysis model and the source code. To narrow this gap, in the future, we need to foresee the implementation complexity for each transition and combine this factor with the genetic evolution. In general, the GA-evolved sequences usually tend to detect mutants earlier than random sequences, and detect more mutants than the random sequences with the same test costs. On the other hand, we observe that GA-evolved sequences based on all-definitions usually do better in mutant detection than the GA-evolved sequences based on all-DU pairs.

In this case study, we observe the deception phenomenon in permuation problem. It causes the genetic algorithm to not being able to widely exploite the search space because it is often trapped into one deceptive attractor and cannot move out from it. As a result, the genetic algorithm usually presents us solutions from one GA run that have similar genotype but with differences in phenotype. As well, since this problem has a multimodal search space and one GA run may be trapped into one mode, the differences between GA runs are expected and observed: different GA runs may present quite different solutions in genotype, and occasionally, one GA run may present solutions from different modes as we observed in section 6.3.4. 
Since the GA-evolved sequences between different GA runs are quite different in genotype, it is not a surprise that the different GA runs show signinficant variance in mutant detection. But in fact, within one GA run, though the GA-evolved sequences are usually very similar in genotype, they still show some variance in the mutant rates because (1) some powerful test cases are arranged slightly differently in the sequence of test cases, and (2) occasionally, we observe quite different test case sequences from two modes within one GA run.

In this case study, we again see the seeded mutants are distributed in an unbalanced way, which makes around one third of the test cases too effective (powerful) at killing mutants. Any sequence invoking a test case in the powerful subset early will have a high mutant rate. That's why we observe significant variance in the mutant rates, and why random sequences can perform well.

\subsection{VCR System Case Study-Results}

For the VCR system, the state machine diagram and all operation contracts are presented in appendices E.3 and F.3. Similarly to previous case studies, we do not only present the average data flow coverage rate and the average mutant detection rate at each test cost, but we also present their minimum and maximum values for each test cost. "mean_TCS" shows the average rate (data flow coverage rate or mutant detection rate) of GA-evolved sequences at each test cost. "min_TCS" and "max_TCS" show their minimum and maximum values. They are compared to the average rate and the minimum and maximum values at each test cost of a set of random test case sequences, shown as "mean_random", "min_random" and "max_random". Note that when reporting on results that have large test cost ranges for the $\mathrm{x}$-axis, we use a logarithmic scale to facilitate observations.

\subsubsection{VCR: Optimization Scheme 1 -Specifying a Maximum Test Cost}

In the initial run, we specify the user specified test cost $T$ to a quite high value (8600, $99.7 \%$ of total transitions) for the purpose of insuring that all solutions will reach $100 \%$ data flow coverage at $T$. Our GA application shortens $T$ to $T^{\prime}=162$ (around $2 \%$ of total cost) for all-definitions and $T^{\prime}=1752$ (around $20 \%$ of total cost) for all-DU pairs, 
respectively. We then try two GA runs with $T$ equal to 86 and 258 (around $1 \%$ and $3 \%$ of total cost) for all-definitions, and other two GA runs with $T$ equal to 862 and $2586(10 \%$ and $30 \%$ of total cost) for all-DU pairs. For the larger values than $T^{\prime}$, our GA application always shortens solutions to values around $T^{\prime}\left( \pm 7 \%\right.$ of $\left.T^{\prime}\right)$. This larger variation than the Ordered Set case study is due to the larger size of the VCR case study (larger number of test cases and data flow elements as reported in Table 5). Since our GA application returns results similar to the ones we get with $T$, we only show results from one GA run (using $T^{\prime}$ ). We provide the GA results from the initial GA run $(T=8600)$ and the smaller values than $T^{\prime}$ that are 86 for all-definitions and 862 for all-DU pairs in Table 21.

Y $O$ - mean_random - min_random

+ max_random

mean_..TCS

$\Delta$ min_TCS

$r$ max_TCS

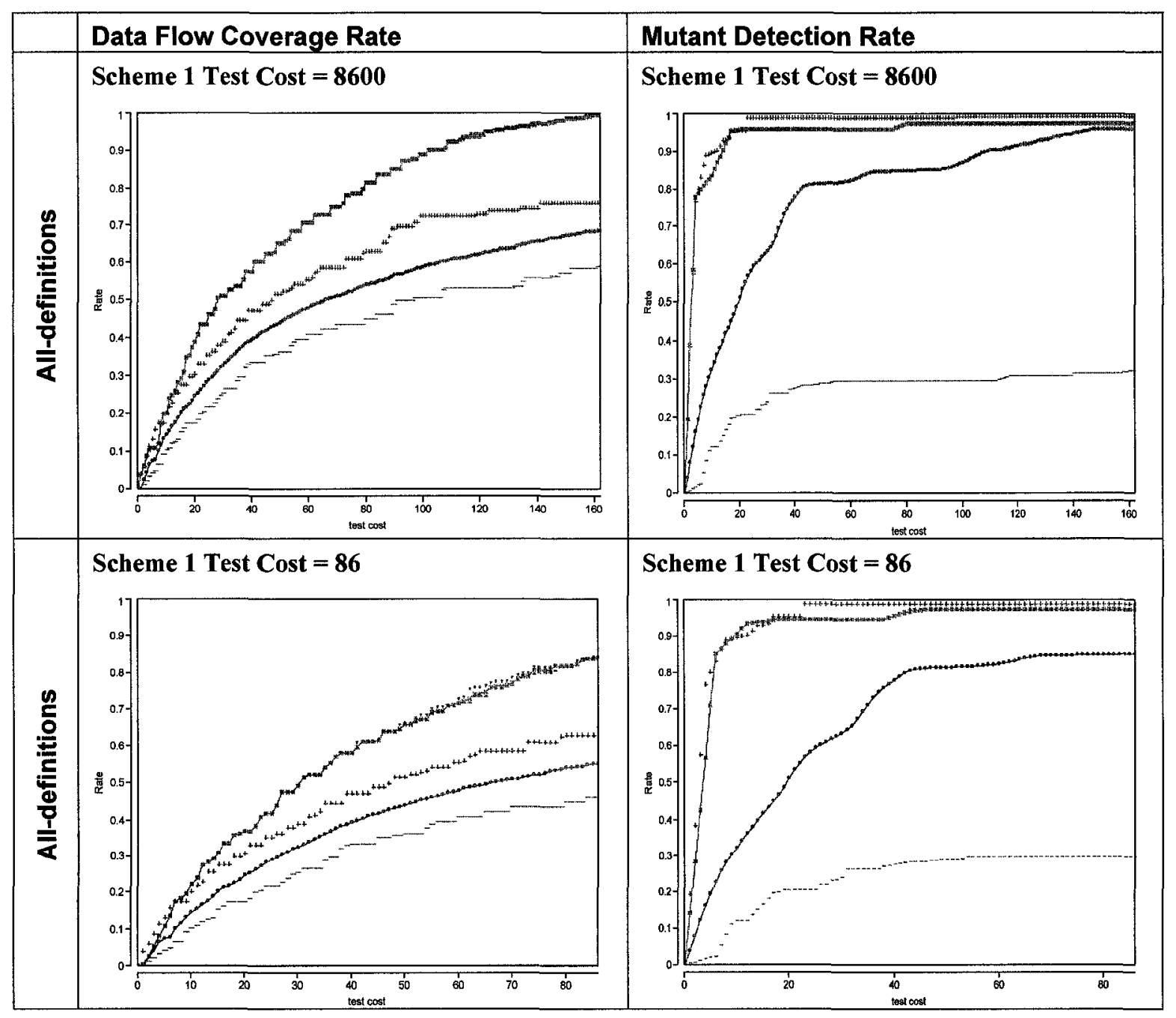




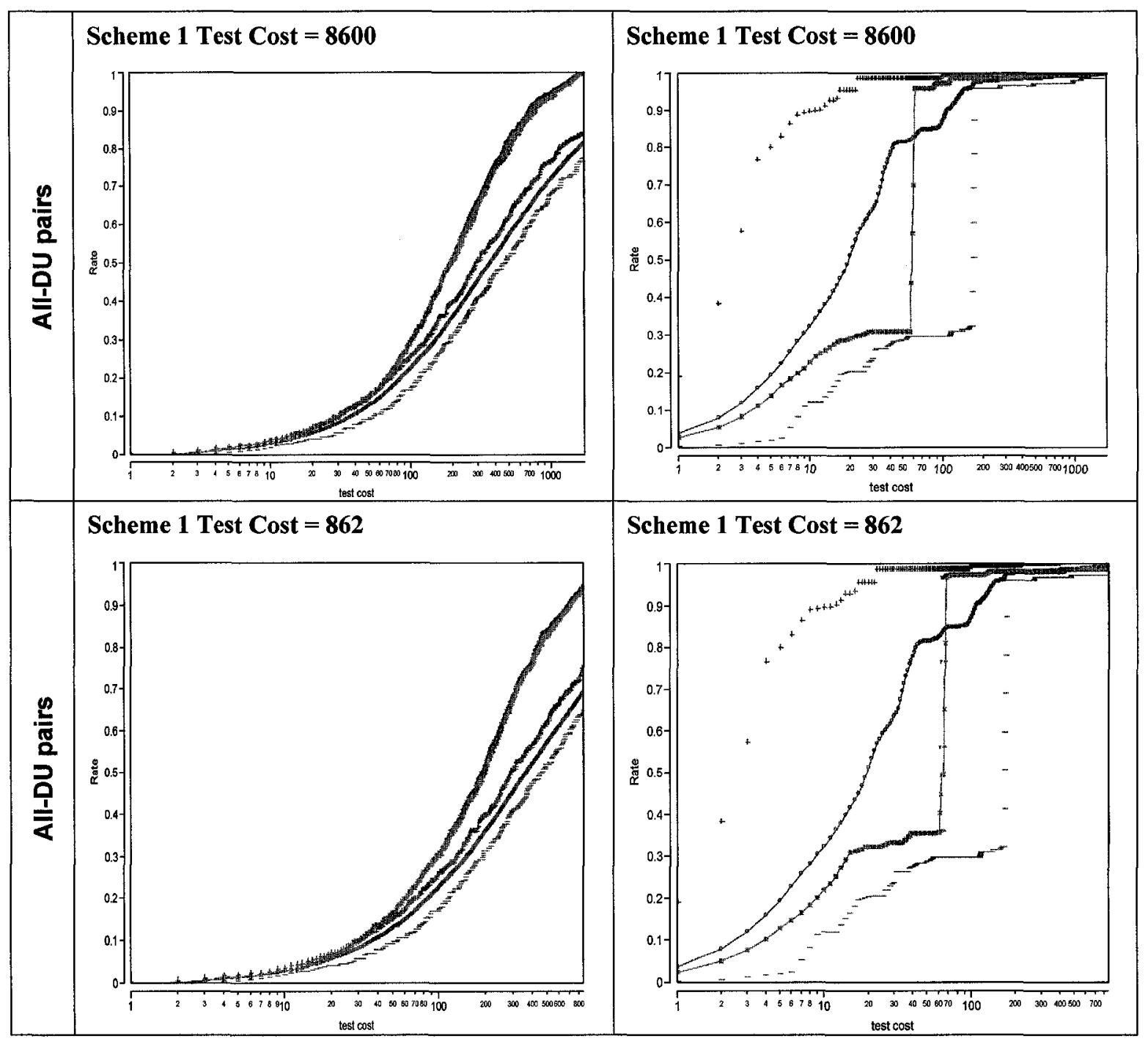

Table 21: Data flow coverage rate and mutant score comparison with GA-evolved sequences and random sequences (VCR-Scheme 1)

From Table 21, the GA-evolved sequences increase their data flow coverage rates quicker, which is the intended behavior, with smaller variance than the random sequences. But the GA-evolved sequences do not show a significant advantage over the random sequences during the first 10 test costs for all-definitions and during the first 30 test costs for all-DU pairs. We identify three reasons. First, we have deceptive attractors as what we mentioned in section 6.3.1. Since there are a lot of deceptive attractors in the huge searching space (size of 1546!), the GA evolution is easyly trapped into a deceptive attractor because of the stochastic factor in the genetic algorithm, which causes the GA converging to a subdomain of the searching space. This case study has 1548 test cases 
with test costs 1 to 8 . Due to this large number, we were not able to find the first level of deceptive attractors, the second level attractors and so on as discussed in section 6.3.1. However, given that the search space is larger than the one for OrderedSet and we observed deceptive attractors when studying OrderedSet, we can reasonably assume that we potentially have many more deceptive attractors for VCR. The second reason is the large number of test cases. This dramatically increases the difficulty for the GA to find the best test cases for the genes at the beginning of the sequence since the searching space is huge. Please recall that we use the weighted cumulative coverage rate as one objective value, which has no way to gurantee to select the best data flow coverage rate at any specific test cost. The third reason is the large number of data flow elements in this case study (the same reason as we discussed in section 6.3.1.1). It takes more time for GAsequences to accumulate the obvious advantage in the data flow coverage rate than the random sequences. For instance, a test case covering 2 definitions or $8 \mathrm{DU}$ pairs more than another test case at a certain test cost (which is common because transitions do not cover large amounts of definitions or uses), let's say cost 2 , shows a $1.1 \%(2 / 184)$ or $0.7 \%(8 / 1219)$ advantage.

Based on all-definitions, the GA-evolved sequences detect around $95 \%$ mutants with the first 20 test costs and then enter a plateau. This means that around 95\% mutants are detected by only three to five test cases. The random sequences detect $80 \%$ mutants with around 40 test costs on average and then their mutant detections increase slowly. Based on all-DU pairs, the GA-evolved sequences detect fewer mutants than random sequences at the first 60 test costs, but they have sharp increments and detect more than 96\% mutants with around 70 test costs. With 70 test costs, based on either data flow criterion, the GA-evloved sequences detect more than 95\% mutants, but the random sequences detect around $85 \%$ mutants. After that, the random sequences detect more mutants with slow increments until around test cost 120 . Then the mutant rates of the random sequences increase again for around 50 test costs and become quite close to the ones of the GA-evolved sequences (the difference of the mutant rates between the GA-evloved sequences and the random sequences is less than 2\%) after 180 to 260 test costs because both GA-evolved sequences and random sequences obtain quite high mutant rate. 
We find that the plateau in the mutant rates of the GA-evolved sequences can be explained in a similar way we explained the plateau in section 6.3.1.2 for the Ordered Set system: 1) there are guard conditions in this case studies, 2) from the study to mutants, we again notice MuJava seeds a lot of mutants that are quite easy to detect and these mutants are distributed in an extremely unbalanced way (see below).

We notice a sharp increment in the mutant rate: steep jump between test costs 60 and 65 from $26.5 \%$ to $91.7 \%$ for all-DU pairs. This is due to a test case from a powerful set of test cases (178 test cases out of a total of 1548). The powerful set of test cases has common characteristics: their mutant detection capability is quite high, range from $70 \%$ to $86.5 \%$; all of them traverse the "Recording" state. From the mutant analysis, we found that of the 175 mutants, 99 mutants are seeded in the constructor and the method calculateNewTapePosition() in Recording.java. Most of these mutants are the easy to kill as they can be detected by any use. Any test case that traverses the "Recording" state will obtain a relatively high mutant detection rate $(>50 \%)$. With combination to the other transitions, the most powerful test case in this set detects $86.5 \%$ mutants. The other test cases in this powerful set usually obtain quite good mutant rates range from $70 \%$ to $85 \%$. It explains the sharp increment in the mutant rate.

We also observe the GA-evolved sequences based on all-DU pairs show a plateau before the sharp increment in the mutant rate. From the analysis, we find that covering more DU pairs does not necessarily result in covering more definitions. If the major mutants are easy to be detected by covering all definitions, the criterion of all-DU pairs that intends to cover more DU pairs does not work well. As a result, we see the plateau of the mutant rate from the GA-evolved sequences base on all-DU pairs before the sharp increment.

The sharp increment again reveals that the mutants seeded by MuJava are not evenly distributed in the source code as what we observed in the previous two case studies. Based on all the three case studies, we encounter the same problem for the mutant distribution that some test cases are so powerful to kill the large number of mutants, these test cases appearance in the sequences significantly change the mutant rate. We would like to suggest that a user be careful to re-select the mutants generated by MuJava with 
consideration of the balance of mutant distribution. In the following studies, we need to keep in mind the sharp increments in the mutant rate both in the GA-evolved sequences and in the random sequences.

\subsubsection{VCR: Optimization Scheme 2-Specifying a Cumulative Coverage Rate}

In this optimization scheme, we specify two user expected cumulative coverage rates (SC), $80 \%$ and $90 \%$ data flow coverage rates based on all-definitions and all-DU pairs. We present the comparison result in Table 22.

$Y \quad 0-$ mean_random - min_random + max_random $x-$ - mean_TCS $\triangle$ min_TCS $Y$ max_TCS

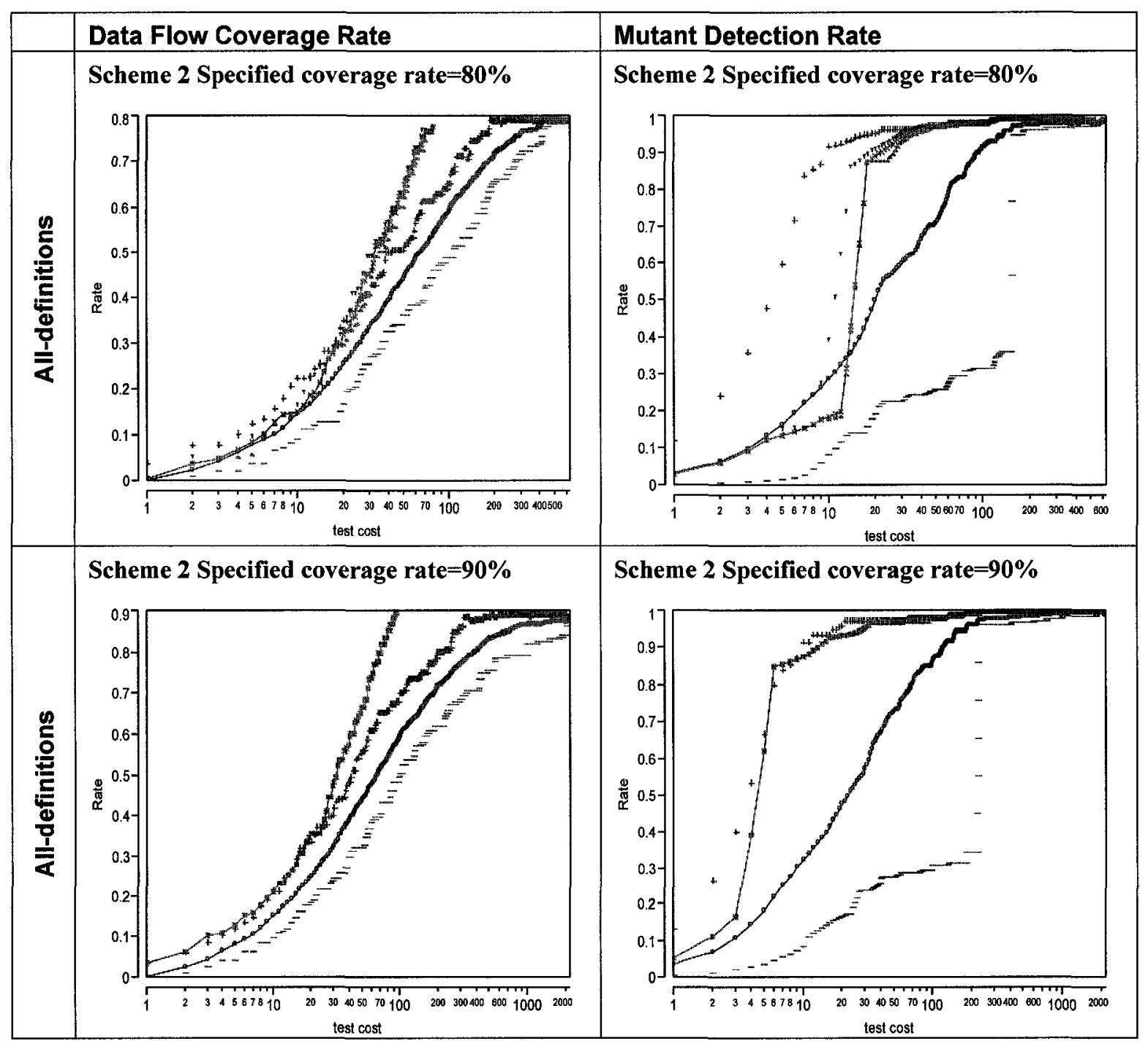




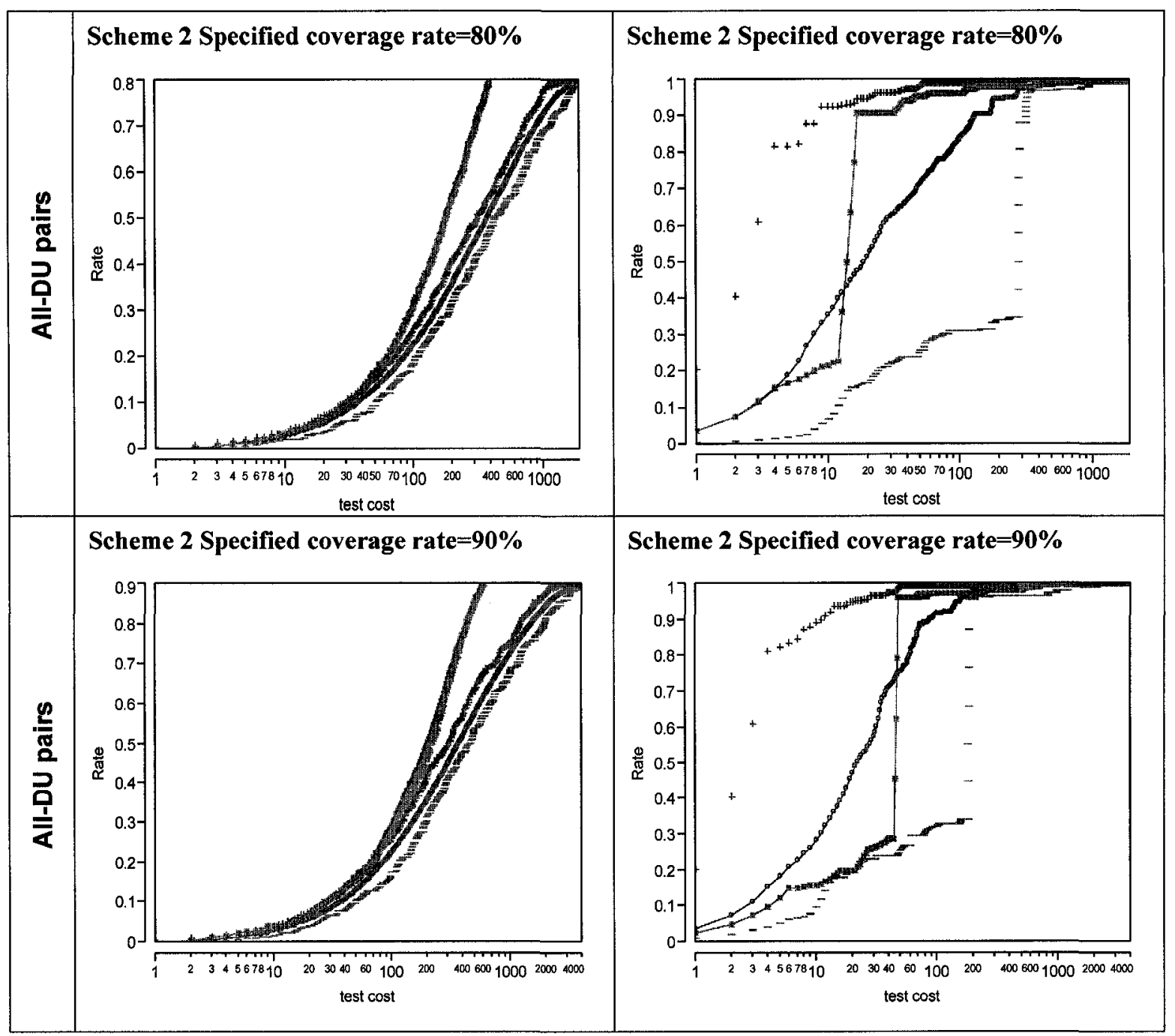

Table 22: Data flow coverage rate and mutant score comparison with GA-evolved sequences and random sequences (VCR-Scheme 2)

With a data flow coverage rate of $80 \%$ or $90 \%$, the GA-evolved sequences based on alldefinitions or all-DU pairs increase their data flow coverage rates quicker than the random sequences. With different data flow criteria, the test costs are significantly different: based on all-definitions, the GA-evolved sequences take 67 to 70,93 to 94 , test costs to reach $80 \%, 90 \%$ data flow coverage rate, respectively; based on all-DU pairs, the GA-evolved sequences take around 383 to 387,672 to 601 , test costs to reach $80 \%, 90 \%$ data flow coverage rate, respectively. In comparison, based on all-definitions, the random sequences take 177 to 501,348 to 2511 , test costs to reach $80 \% .90 \%$ data flow coverage rate, respectively; based on all-DU pairs, the random sequences take 1178 to 1812 , 
around 2169 to 3886 , test costs to reach $80 \%, 90 \%$ data flow coverage rate, respectively. For the mutant rates, the GA-evolved sequences based on all-definitons reach $97.4 \%$ and $97.6 \%$ mutant rates when they achieve the data flow coverage goals $80 \%$ and $90 \%$, respectively; the GA-evolved sequences based on all-DU pairs reach $98.6 \%$ and $98.7 \%$ mutant rates when they achieve the data flow coverage goals $80 \%$ and $90 \%$. From the variance analysis, we find the random sequences have much larger variance in terms of both data flow coverage rate and mutant detection rate.

Based on this scheme, we observe that the GA-evolved sequences, based on alldefinitions and $S C=80 \%$, have similar mean values as the mean values of random sequences for the first 15 test cost values, but the GA-evolved sequences, based on alldefinitions and $S C=90 \%$ have similar mean values as the maximum values of random sequences for the first 15 test cost values.

When we study the GA-evolved sequences for $S C=80 \%$ in all-definitions, we find there are two sets of sequences which will lead to two different sub domains of the search spaces (multimodals as we mentioned in section 6.3.1 and we also observed one GA exploiting two multimodals in section 6.3.4). One set reaches $S C$ with a shorter test cost but its cumulative data flow coverage rate is not worse than the other set. The other set has better weighted cumulative data flow coverage rates but it reaches $S C$ with longer test costs. So we see some legible variance in the data flow coverage rates.

When we study the GA-evolved sequences for $S C=90 \%$ in all-definitions, we find that the GA-evolved sequences have very similar genotypes again (these test case sequences come from the same sub domain of seaching space), and by the GA stochastic factor, the first test case is one of equivalent test cases doing best in the data flow coverage. So they show around 3\% advantage than the random sequences in the data flow coverage rate in the first 15 test cost values.

About the mutant rates, we already discussed the plateau and the sharp increment in section 6.4 .1 that is caused by unbalanced mutant distribution. So we do not repeat it again here or in the other optimization schemes. 


\subsubsection{VCR: Optimization Scheme 3-Specifying 100\% Cumulative Coverage Rate and Maximum Available Test Cost}

In this optimization scheme, we do not specify any user expected data. This scheme simply uses $100 \%$ cumulative coverage rate and $100 \%$ of total transitions. Since all GAevolved sequences and all random sequences take $100 \%$ of total transitions, we will not observe the non-monotonic situation. The comparison result is given in Table 23: $\mathrm{SC}$ is the user specified coverage rate $100 \%$ and $\mathrm{T}$ is $100 \%$ test costs 8622 .

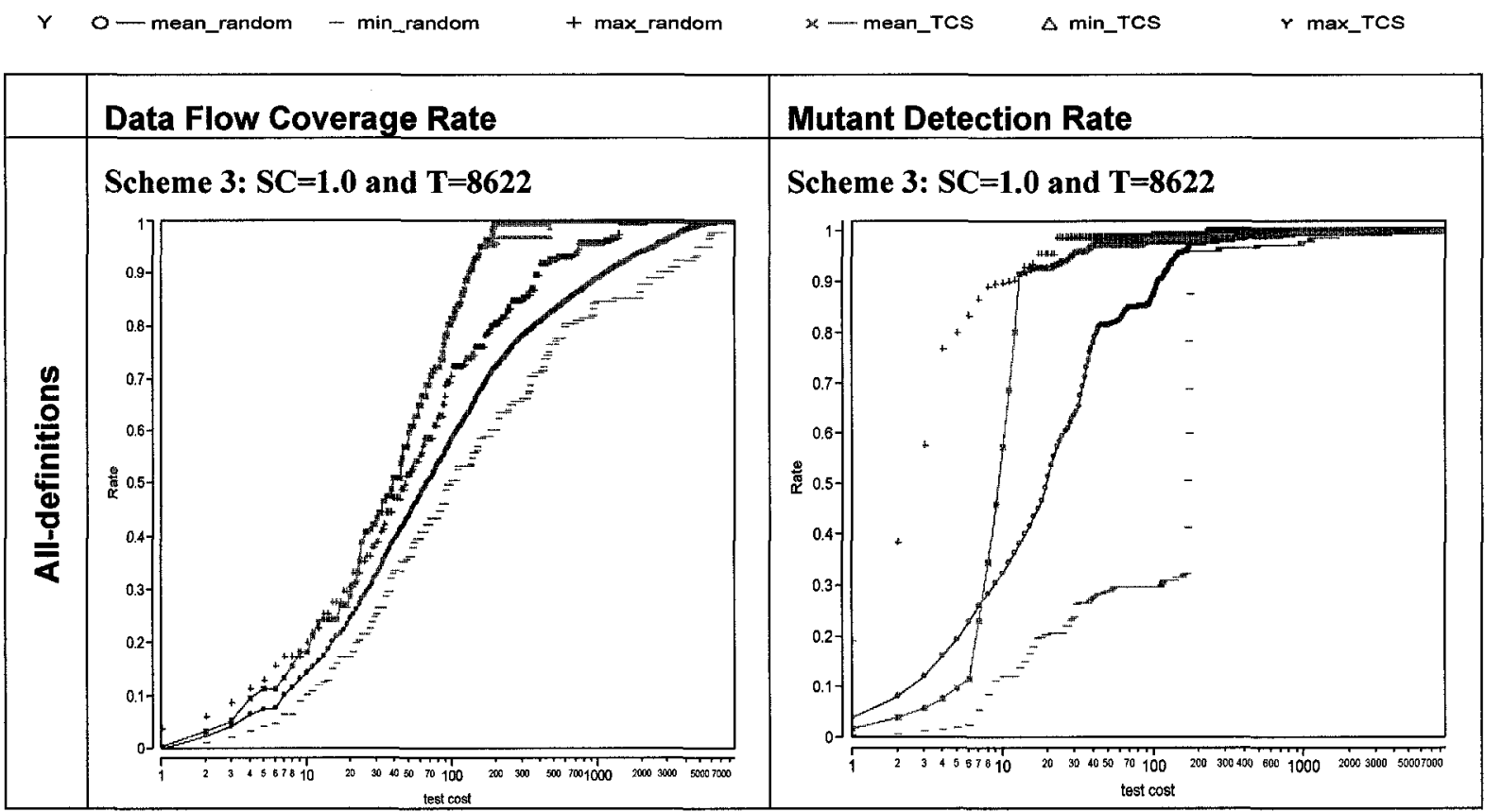




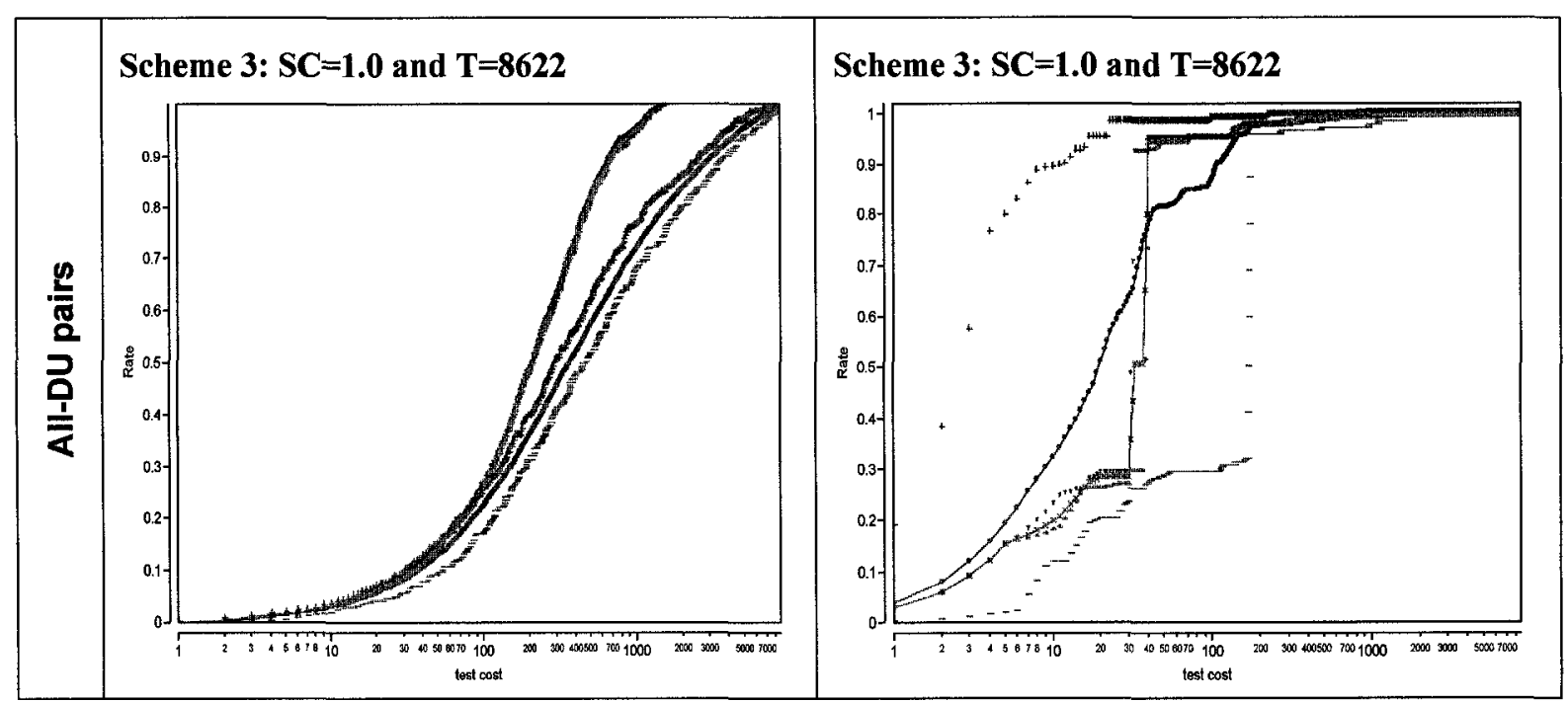

Table 23: Data flow coverage rate and mutant score comparison with GA-evolved sequences and random sequences (VCR-Scheme 3)

With all test costs (8622) and the default coverage rate of $100 \%$, the GA-evolved sequences increase their data flow coverage rates quicker than the random sequences with much smaller variance. The GA-evolved sequences cover $100 \%$ of all definitions with test costs 151 and $100 \%$ of all DU pairs with test costs from 1591 to 1804 . From a comparison of the mutant rates, the GA-evolved sequences detect more mutants than the random sequences at the first 150 test costs. The GA-evolved sequences based on alldefinitions detect $98.1 \%$ of mutants when they cover $100 \%$ of all definitions. The GAevolved sequences based on all-DU pairs detect $100 \%$ of mutants when they cover $100 \%$ of all DU pairs. In general, based on either data flow criterion, the GA-evolved sequences detect $96 \%$ mutants with taking test costs 30 to 70 , but the random sequences detect $96 \%$ mutants with taking test costs 120 to 170 .

Again, we observe the GA-evolved sequences based on all-definitions perform better than the ones based on all-DU pairs in mutant effectiveness. For the same cost, the GAevolved sequences based on all-definitions usually have better mutant rates than the ones based on all-DU pairs. Though there are sharp jumps for both sequences, the GA-evolved sequences based on all-definitions have the sharp jump earlier than the ones based on allDU pairs. 


\subsubsection{VCR: Optimization Scheme 4-Specifying a Maximum Test Cost and a Minimum Cumulative Coverage Rate}

In this optimization scheme, we need to specify a maximum test cost and a minimum cumulative coverage rate. Since our GA application could heuristically shorten the test cost to a value that satisfies the minimum cumulative coverage rate, we simply specify a relatively large test cost combined with the data flow coverage rate $S C 80 \%$ and $90 \%$. In the initial GA runs, we specify test costs 6898 and 7760 for all-definitions and all-DU pairs to make sure almost all solutions could reach the minimum $S C$ at the end of test case sequences.

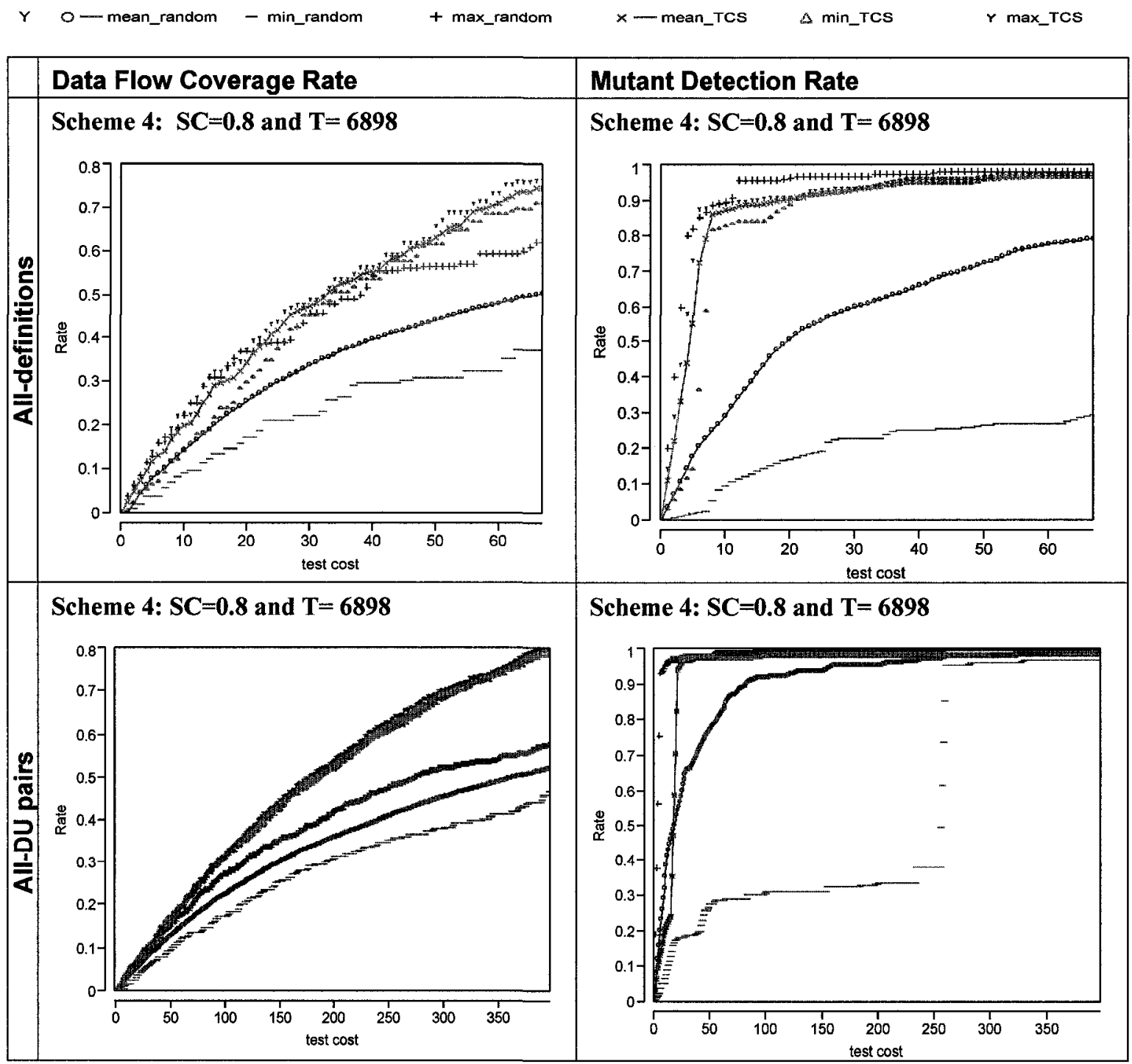




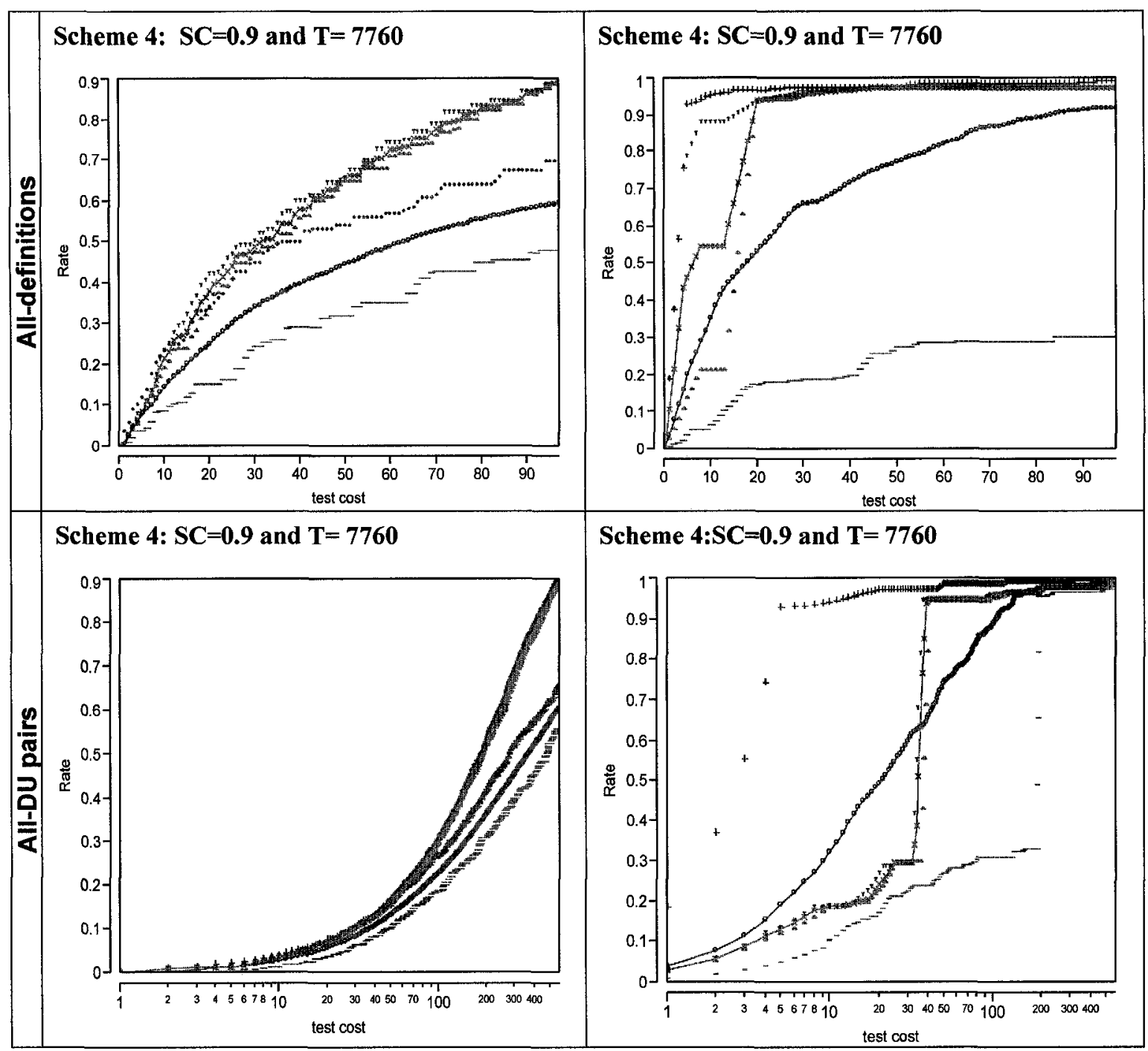

Table 24: Data flow coverage rate and mutant score comparison with GA-evolved sequences and random sequences (VCR-Scheme 4)

From Table 24, we can see that, with any combination of the user input data, the GAevolved sequences increase their data flow coverage rates quicker than the random sequences and reach the specified data flow coverage rate with less test cost than the user-specified test cost. To cover $80 \%$ and $90 \%$ of all definitions, the GA-evolved sequences take, at the best, 65 test costs and 90 test costs respectively, so $T^{\prime}$ are modified to 67 and 92 respectively (recall $T^{\prime}$ is the average of test costs where the GA-evolved sequences reach SC). To cover $80 \%$ and $90 \%$ of all-DU pairs, the GA-evolved sequences take, at the best, 386 test costs and 554 test costs respectively, so $T^{\prime}$ is modified to 398 and 574 respectively. The GA-evolved sequences have very small variances in the data 
flow coverage rate. In comparison, the random sequences have large ones. We notice the GA-evolved sequences based on all-definitions have relatively large variance for the data flow coverage rates with the different inputs. After checking the GA-evolved sequences, we find that in the two GA runs, the GA algorithm occasionally exploites two modes of the search space and preserves solutions from these two subdomains. So we observe the variance in the data flow coverage rates. In fact, we also have the same observation in section 6.3.4.

From the mutant detection rate, the GA-evolved sequences based on all-definitions usually have much better mutant rates from test costs 20 to the end of sequences and much smaller variances than the random sequences; the GA-sequences based on all-DU pairs have better mutant rates from test cost 40 to around 200 and smaller variances than the random sequences, and after test costs around 200, the GA-sequences and the random sequences have very similar mutant rates.

Since our GA application shortens $T$ to $T$ ' for all-definitions and all-DU pairs, we apply the round value of $T^{\prime}$. The larger one is used to verify if our GA application consistently shortens $T$ to $T^{\prime}$ if $T$ is large enough to satisfy $S C$. The smaller one is used to verify our GA application will present a new $S C^{\prime}$ and tell the user the time budget is not enough to satisfy $S C$ but the user could expect $S C$ ' within $T$ '. So in the following GA runs, for alldefinitions, we specify test costs 43 and 431 for $S C=80 \%$, and test costs 86 and 431 for $S C=90 \%$; for all-DU pairs, we specify test costs 345 and 862 for $S C=80 \%, 431$ and 862 for $S C=90 \%$. Since the results of values larger than $T^{\prime}$ are very similar to the initial runs, we do not present the comparison results here. In Table 25, we present the comparison results based on the combinations of smaller than $T^{\prime}$ test costs and two cumulative coverage rates. 


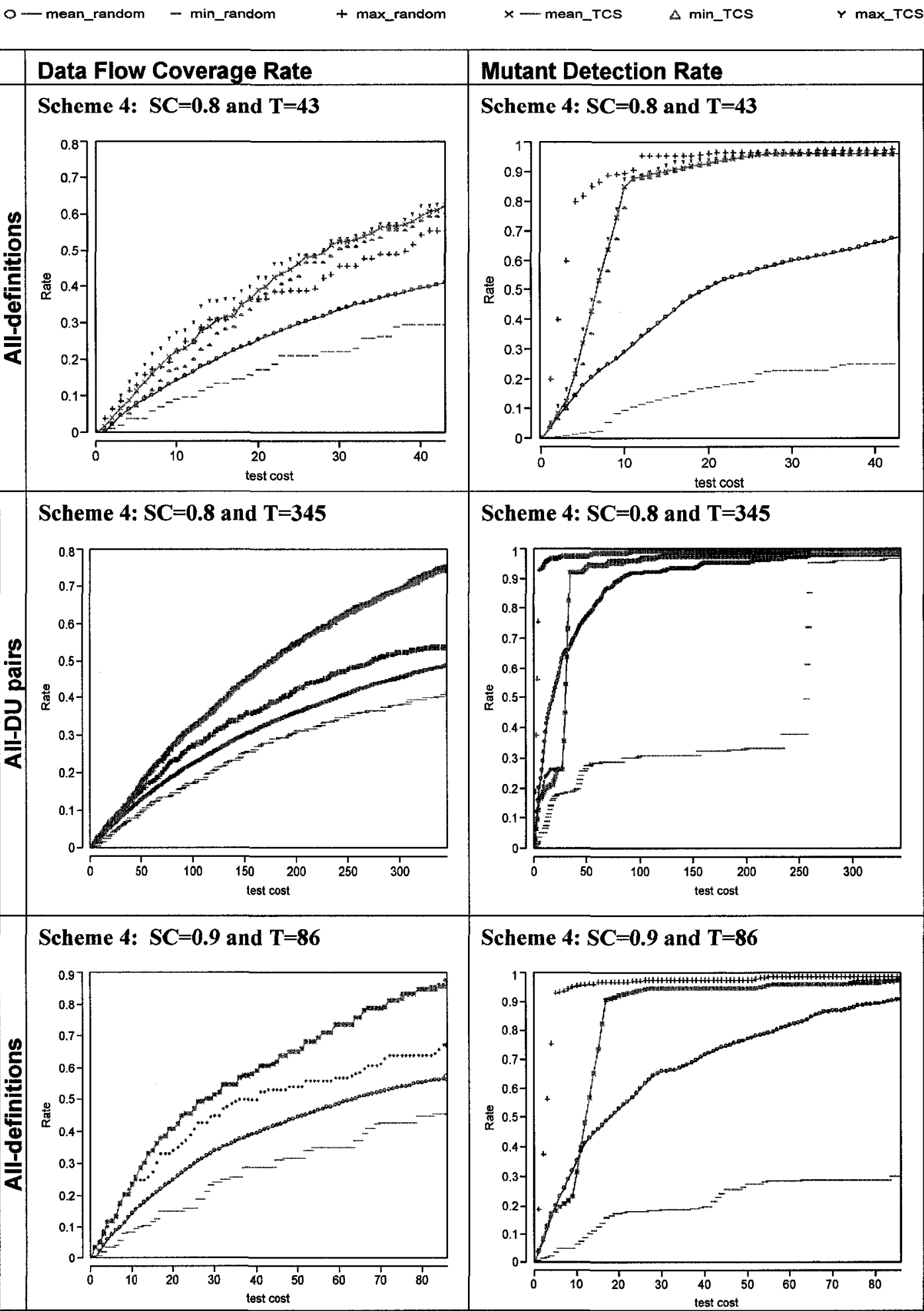




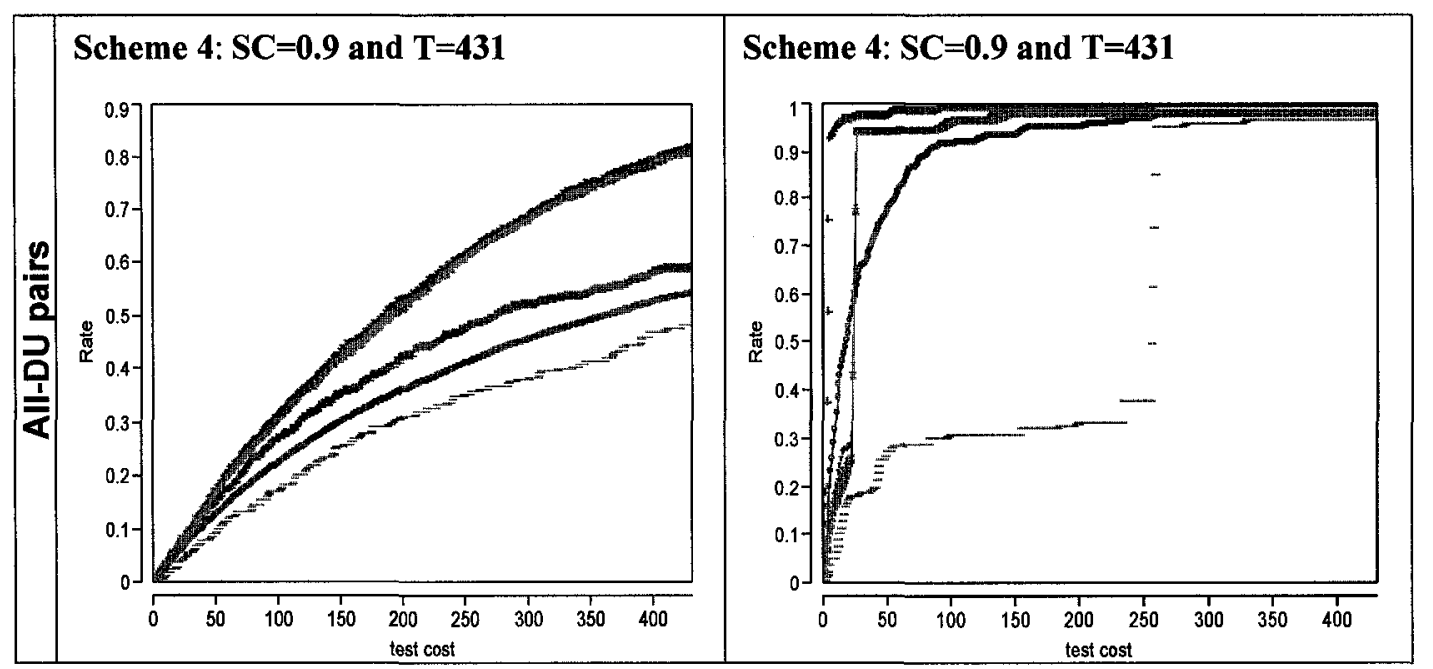

Table 25: Data flow coverage rate and mutant score comparison with GA-evolved sequences and random sequences (VCR-Scheme 4), new Ts.

From the comparison results, we can see that the GA-evolved sequences reach $65 \%$ and $87 \%$ of all definitions with test costs 43 and 86 , respectively, and $76 \%$ and $82 \%$ of all DU pairs with test costs 345 and 431, respectively. They have very small variance in the data flow coverage rates. From the analysis of mutant detection rates, the GA-evolved sequences based on all-definitions do not necessarily detect more mutants than the random sequences at the beginning 10 test costs, but they increase quickly after that and outperform the random sequenes to the end of sequences; the GA-evolved sequences based on all-DU pairs also do not necessarily detect more mutants than the random sequences at the first 30 test costs, but they increase very quickly and outperform the random sequences from test costs 30 to around 200. The GA-evolved sequences based on either data flow criterion always have much smaller variances than the random sequences.

\subsubsection{VCR: Study of the variance between GA runs}

From this case study, we already see some variances between different GA runs. Similarly the Ordered Set system, we studied the variances between each genetic run. We executed 20 genetic runs with evolution scheme 3 based on all-definitions. After that, we randomly picked one GA-evolved sequence from each genetic run to form a set of the GA-evolved sequences. These 20 GA-evolved sequences are compared with 20 random 
sequences. We present our comparison result in Table 26, where we show all data: data flow coverage rate and mutant detection rate on each test cost for each test case sequence, as well as the minimum, maximum and average mutant rates of 20 test case sequences.

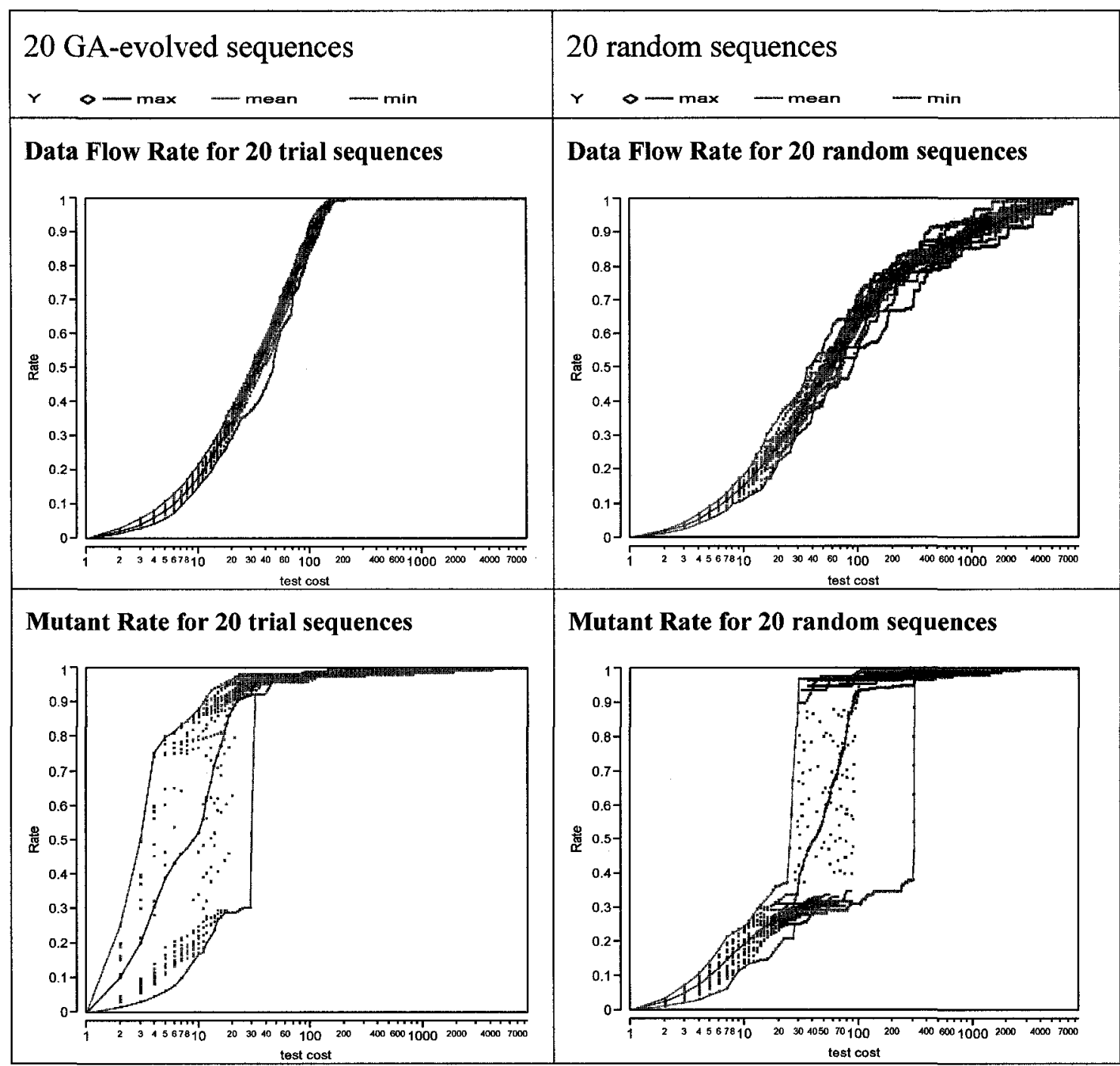

Table 26: Data flow rate and mutant rate in comparison with 20 GA-evolved sequences from 20 trials and 20 random sequences -VCR System

From Table 26, we find that all the data flow coverage rate curves of 20 GA-evolved sequences increase much quicker than the random sequences and reach $100 \%$ data flow coverage rate at around 150 test costs (instead of an average cost of 6000 for random sequences). The variance between the $20 \mathrm{GA}$-evolved sequences is very small. However, 20 random sequences have a great deal of variance in the data flow coverage rate. The 20 GA-evolved sequences cover $100 \%$ of all definitions at the shortest test cost 144 and the 
longest test cost 167 , while the best random sequences cover $100 \%$ of all definitions with the test cost 2410 .

In this figure, we could see some apparent variances in the data flow coverage rate for 20 GA trials. We believe that the deceptive attractors that we mentioned in section 6.3.1, the large search space and the large number of data flow elements, all together, cause the GA evolutions to show variance. With regards to the variances in the data flow coverage rate, the GA-evolved sequences are still much better than the random sequences, increasing coverage faster and reaching full coverage earlier. In comparison with the random sequences, the GA-evolved sequences have very small variance in the data flow coverage rates.

The average mutant rates of the GA-evolved sequences have a $30 \%$ or more advantage over the random sequences during the first 50 test cost values. The mutant rate of the GA-evolved sequences reaches $97 \%$ at test cost value 45 with a very small variance of $1 \%$. However, the mutant rate of the random sequences reaches $90 \%$ at test cost value 90 with a quite large variance (with the minimum mutant rate of $31 \%$ and the maximum mutant rate of 97\%). Even though, in this case, the mutants are not evenly distributed, the GA-evolved sequences still detect them earlier than random sequences.

From this study, we obtain similar conclusion as the OrderedSet system: first, though the deceptive attractors exist, the GA-evolved sequences have very small variance in the data flow coverage rates (in phenotype); second, because the GA-evolved sequences are different in genotype, their mutant results show significant variance. In comparison with the random sequences, the GA-evolved sequences show smaller variance in data flow coverage rate and mutant rate, as well as the tendency to detect mutants earlier.

\subsubsection{VCR: Summary of Results}

The VCR system has a huge search space, which contains 1548 ! solutions. We only obtain relatively better solutions at the $500^{\text {th }}$ generation. Usually, the GA-evolved sequences have similar data flow coverage rates as random sequences during the first 5 to 10 test cost values for all-definitions and during the first 10 to 20 cost values for all-DU 
pairs. Because of reasons such as the presence of deceptive attractors, the stochastic factors in the genetic algorithm, the large number of data flow elements and the way we defined the objective functions, the test cases with better data flow coverage rate, which appear early in random sequences, are not often arranged at the beginning of GA-evolved sequences. But we can not say the our GA totally fails since we look for complete test case sequences rather than only focussing on the beginning part of test case sequences. We can only say it is the best hope that the GA-evolved sequences do better in data flow coverage rate anywhere possible (anywhere it is less than 100\%). But such an advantage in data flow coverage does not necessarily appear at any test cost value, especially at the beginning part of a sequence, because of many reasons we mentioned before. In general, our GA-evolved sequences do better than the random sequences in the data flow coverage rates in the majority part of test case sequences where the data flow coverage rate increases from zero to the user defined coverage rate or the full coverage rate.

In our comparisons with four optimization schemes, the data flow coverage rates of the GA-evolved sequences increase much more quickly than those of the random sequences. The GA-evolved sequences reach the user specified coverage rate or the full coverage rate with less costs, or cover more data flow rates within the limited user specified costs, than random sequences.

In the comparison of the mutant rates, both the GA-evolved sequences and the random sequences have extremely sharp increments in the mutant rate (though we do not see that from the average curve of random sequences just because the values are averaged). By studying mutant distributions, we identify the mutants generated by MuJava are distributed in an extremely unbalanced way: a majority of mutants are seeded into one method, and a minority of mutants are scattered into the other methods. Such an unbalanced mutant distribution, causing the extremely sharp increases in mutant curves, can not represent likely defects in the source code introduced in the implementation phase. As a result, we do not think we could conclude something meanful or reliable based on the comparison of the mutant rates between the GA-evolved sequences and the random sequence. Same as the past experience of the first two case studies, we again see the side effect of the automated mutant generation tool. We strongly recommend that the 
users of such a tool check and re-evaluate the generated mutants one by one, and consider the mutant distribution cautiously. As well, we have to point out it may be a quite time consuming thing to do so since the automated tool usually generates a quite large number of mutants.

\subsection{Case Study Conclusion}

From all three case studies, we observed that the GA-evolved sequences increase their data flow coverage rates much quicker than the random sequences. For a small or medium test suite (the Cruise Control or the Ordered Set case studies), the data flow coverage rate difference between the GA-evolved sequences and the random sequences may increase to $30 \%$ to $40 \%$ with the first $10 \%$ of total test cost. For a larger test suite (the VCR case study), the data flow coverage rate difference between the GA-evolved sequences and the random sequences may increase to $30 \%$ to $40 \%$ within the first $5 \%$ of total test costs.

Based on the all-definitions criterion, for the small (Cruise Control), medium (Ordered Set) and large (VCR) test suites, the GA-evolved sequences can cover all definitions with, respectively, $50 \%$ of total test cost for the small, around $10 \%$ for the medium and around $2 \%$ for the large. In comparison, the random sequences can cover all definitions with, respectively, $90 \%$ of total test cost for the small, $70 \%$ for the medium and $50 \%$ for the large.

Based on the all-DU pairs criterion, for the small, medium, and large test suites, the GAevolved sequences can cover all DU pairs with, respectively, $90 \%$ of total test cost for the small, $50 \%$ for the medium and $20 \%$ for the large. In comparison, the random sequences can cover all DU pairs with, respectively, $99 \%$ of total test cost for the small, $98 \%$ for the medium and $90 \%$ for the large.

In the later two case studies, we observed that the GA-evolved sequences from one GA run look quite similar to each other in genotype (the subset of test cases and their orders), and the GA-evolved sequences from different GA runs are quite different in genotype. We discussed that a likely reason is that permuation problems are deceptive, a problem 
revealed by [55], that is that the GA search may be trapped into different deceptive attractors (local optima) in different GA runs and a GA search/run may be limited within one deceptive attractor and it is hard to move out from it for better exploiting the search space. In combination with other stochastic factors in the genetic algorithm (such as the crossover position, mutation position, etc), we may see some differences in the data flow coverage rates based on different GA runs, shown in the study to the variances between $20 \mathrm{GA}$ runs in section 6.3.5 and section 6.4.5.

With regard to the mutation detection capability, in general, we find the GA-evolved sequences usually can detect most of the mutants by covering all definitions and can detect all mutants by covering all DU pairs. In general, for all three test studies, the GAevolved sequences can detect more mutants or detect mutants earlier than the random sequences with the same costs except sometimes at the very beginning part of sequences. We notice in all case studies that mutants are distributed in an unbalanced way, i.e., most mutants are seeded into methods with more computation statements. The unbalance mutant distribution causes the test cases polarization in terms of mutant rates as a powerful set and an un-powerful set. As a result, the curve of mutant rate is quite sensitive to the appearance of the test case from the powerful set. The reason is that MuJava seeds mutants exhaustively and blindly without bias. But in fact, it gives more preference to statements involving more computations for mutants seeding. From another perspective, it reveals the gap between the design model and the code implementation. To confirm our discovery of the mutant rate sensitiveness to the mutant distribution, in the first case study, we tried to re-select the mutants so that they are more evenly distributed in the source code. The comparison results show the GA-evolved sequences usually detect mutants earlier and in larger number than random sequences. Acknowledging the likely existence of unbalanced mutant distributions, we studied and analyzed the second and the third case studies. We noticed that the same problem exists for all case studies and causes the curve of mutant rate to be quite sensitive.

We also looked at data flow rate differences and mutant rate differences between different GA runs, possibly due to deception, to understand possible variations. For the second and the third case study, we used one optimization scheme and 20 GA runs to 
obtain 20 GA-evolved sequences (one was selected per run). The comparison of 20 GAevolved sequences with 20 random sequences shows: first, though deception exists, the GA-evolved sequences have very small variances in the data flow coverage rates; second, though the GA-evolved sequences show relatively large variations in mutant detection, their variations are still smaller than the ones of random sequences and the GA-evolved sequences tend to detect mutants earlier and in larger numbers than the random sequences.

When comparing the two criteria, all-definitions and all-DU pairs, we find that to reach the same coverage goal, the costs for different criteria are quite different. To reach the same coverage rate, all-definitions costs much less than all-DU pairs. Especially the difference is huge for a medium or large scale problem. In terms of effectiveness, we find that all-definitions is effective to detect most of the mutants seeded by MuJava. Covering all definitions detects more than $87 \%$ mutants for the small problem, $99.8 \%$ for a medium problem and $98.1 \%$ for a large problem with test costs around $46 \%, 10 \%, 2 \%$ respectively. Covering all DU pairs detects all mutants for all problems with test costs around $89 \%, 72 \%, 20 \%$ respectively. By comparing GA-evolved sequences based on those two criteria with the same set of random sequences, we usually observe that GAevolved sequences based on all-definitions usually detect more mutants than the GAevolved sequences based on all-DU pairs at the same cost.

Based on our comparison results of the three case studies, our answers to the research questions are: 1. the data flow information derived at the model level can help testers to identify an execution order of test cases, and covering more data flow information as early as possible potentially helps testers detect defects earlier; 2. the GA-evolved test case sequences, based on the data flow information at the model level, detect mutants in the source code earlier than random sequences, and with a limited test cost the GAevolved sequences usually detect more mutants than random sequences; 3 . the GAevolved test case sequences have relatively small variances in terms of mutant rate, whereas we observe large varinces of mutant rates for random sequences, and even though the GA-evolved test case sequences may not always detect more mutants than random sequences at every test cost value, they tend to do better than random sequences overall, especially within a limited budget. 
As a general conclusion, we believe that the genetic algorithm can help software testers order test cases based on data flow information extracted from operation contracts, and the software testing design can therefore start as early as possible, such as in the analysis phase. The GA-evolved sequences can satisfy a data flow criterion much earlier than random sequences. From the comparison results of three case studies, we believe that the GA-evolved test case sequences have more potential to detect faults earlier than the random sequences or to detect more defects within a limited time. 


\section{CONCLUSION}

The objectives of this research are: to help software tester improve the testing effectiveness by scheduling test cases in an order such that test cases covering large amounts of data flow are executed first, with the hope to reveal faults early; to help software tester select a subset of test suite and order the selected test cases to satisfy some constraints, such as a tight time resource and an expected data flow coverage rate. This research pays its attention to state-based software testing by presenting a general method to order test cases derived from a UML state machine diagram. We applied a heuristic search algorithm - the genetic algorithm to optimize the order of test case sequences by maximizing the increments of data flow coverage rate.

In theory, to start the testing preparation work as earlier as possible, a tester could start from the data flow information contained in the operation contracts from the design results like UML design model rather than the implementation results like source code. So in this thesis, we take the data flow information contained in the design model rather than the implementation as the input of the genetic algorithm to order test cases, and as well, we keep in mind there are gaps between the design model and the implementation (models are abstractions).

Summarized in one sentence, we presented a general method to order test cases, derived from a UML state machine diagram, by using a heuristic search algorithm to maximize the data flow coverage, derived from operation contracts, as earlier as possible. Our methodology consists of three steps: the first step is that a test suite is derived based on a given state machine diagram and criterion, in which each test case contains one test path that is a sequence of transitions. The second step is to identify the definitions and uses of class attributes, parameters and associations from operation contracts of each action in each transition by using the rules defined by Briand et al [5]. This step tries to reveal the potential data flow in the implementation on which the software testing will be executed. Lastly, a multi-objective genetic algorithm is used to order test cases to cover data flow 
information as quickly as possible and satisfy constraints as early as possible if applicable.

In our case studies, based on the characteristics of the test suite that satisfies the modified transition tree criterion, derived from a state machine diagram with guard conditions, we used a fixed length chromosome to do optimization. With different optimization goals, we solved the optimizations with multiple objective functions by using the multiobjective genetic algorithm - SPEA2. To evaluate the test case sequences arranged by the genetic algorithm based on a specific data flow criterion, we compared them with the test case sequences arranged randomly, in terms of data flow coverage rates and mutant detection effectiveness. From the different data flow criteria, we select two of them, alldefinitions and all-DU pairs.

Based on our three case studies, we observed the following with respect to data flow coverage:

- The GA-evolved sequences usually cover data flow elements quicker than random sequences if the given cost is enough to cover all data flow elements, or the GAevolved sequences cover more data flow elements than the random sequences if the given cost is not enough to cover all data flow elements;

- The average data flow coverage rates of GA-evolved sequences almost outperform the ones of random sequences everywhere with a small number of exceptions (for instance, we see similar coverage rates at the very beginning part of the sequence for a medium or large scale problem);

- Usually some test cases are equivalent in the number of data flow coverage rates but they may have quite different mutant detection capabilities;

- With multiple genetic runs for the same optimization goals, we observe quite different test case sequences though their data flow coverage rates may not be very different. For this observation, we analyze and reveal the deceptive attractors in the permuation problem; 
- Based on the study of the variance between 20 GA runs, we realize the variance is not so significant though the deceptive attractors may affect the genetic evolution results.

In terms of mutant detection effectiveness, we observed the following:

- We find the GA-evolved test case sequences usually detect more mutants with less variance at the beginning of sequences than the random test case sequences but not necessarily detect more from the very first test cost values;

- Though the random sequences usually have very good maximum values of mutant rates, no random sequence systematically outperforms the GA-evolved sequences since the maximum values come from a set of random sequences at different costs and these maximum values do not correspond to a specific test case sequence;

- The mutants seeded by MuJava are distributed in an unbalanced way, such that the marjority of mutants are seeded into the statements with heavy computations and a large number of these mutants result in too powerful (effectiveness) subsets of test cases;

- The test cases that are equivalent in terms of data flow coverage are not necessarily equivalent in terms of mutant detection capability, so the GA-evolved sequences often show significant difference in the mutant rates;

- The GA-evolved sequences based on all-definitions usually perform better than the GA-evolved sequences based on all-DU pairs, in other words, usally, detecting more mutants with less costs;

- From the study to the mutant distribution (unbalanced and balanced), we realize the mutants distribution significantly affects the effectiveness comparison results and MuJava way of blindly seeding mutants may cause the mutant detection rates to be difficult to compare. With a balanced mutant distribution, the GA-evolved sequences usually do better than random sequences in effectiveness. 
- Based on the study of the variance between $20 \mathrm{GA}$ runs, we realize based on unbalanced mutants, the variance in effectiveness may be quite significant between GA runs.

Based on the observations and conclusions from the three case studies, we have three considerations and one lesson for the future work. First, there is the existing gap between model design and the implementation: models are abstractions. This gap causes some significant variance in the effectiveness. The genetic algorithm itself has no way to identify the gap. In the future work, some characteristics of the system, such as the (expected) method implementation complexity, or the importance of specific behaviors from an analysis point of view, should be accounted for. Second, SPEA2 does not uses the distance between solutions in terms of genotype, so the GA search may be easily trapped into one or two peaks in a multi-modal search space. In the future work, we will put some effort to use the genotype and hope the GA solutions may have more diversity in genotype. Third, other meta-heuristic search methods that keep a pool of solutions, such as ant colony optimization, grouping genetic algorithm, etc., will be applied and compared for our test case ordering problem. We could also compare our multi-objective GA with heuristics that only manipulate one solution at a time, such as hill climbing.

Based on the analysis of mutant rates in the three case studies, we find that the mutant distribution may significantly affect the comparison results. Our studies reveal that the automated mutant generation tool helps researchers generate mutants automatically and exhaustively without human bias, but its characterisitics may cause the mutant rates to be artificially similar (e.g., because of easy to kill mutants). A researcher must be careful when using a tool to generate mutants since these mutants may be distributed in an unbalanced way (statements involving heavy computations will be seeded with more mutants than other statements), making a lot of mutants pretty easy to kill. 


\section{REFERENCE}

1. J.Offutt, Y.Xiong, and S.Liu. Criteria for Generating Specification-based Tests. in Fifth IEEE International Conference on Engineering of Complex Computer Systems (ICECCS '99). 1999. Las Vegas, NV.

2. J.Offutt, et al., Generating Test Data From State-based Specifications. Software Testing, Verification and Reliability, 2003. 13(1).

3. L.C.Briand, Y.Labiche, and Y.Wang. Using Simulation to Empirically Investigate state Coverage Criteria based on Statecharts. in Proceedings of ACM International Conference on Software Engineering (ICSE'04). 2004. Edinburgh, Scotland, UK.

4. B.Meyer, Object-oriented software construction. second ed. 1997: Prentice Hall PTR.

5. L.C.Briand, Y.Labiche, and Q.Lin. Improving State-Based Coverage Criteria Using Data Flow Information. in Proc. of IEEE International Conference on Software Reliability Engineering. 2005. Chicago, USA,.

6. D.Goldberg, Genetic Algorithm in search, optimization \& machine learning. 1989: Addison Wesley.

7. R.Haupt and S.E.Haupt, Practical Genetic Algorithms. 1998, New York: A Wiley-Interscience Publication.

8. Schaffer, J.D. Multiple objective optimization with vector evaluated genetic algorithms. in In Proceedings of the First International Conference on Genetic Algorithms. 1985.

9. E.Zitzler, M.Laumanns, and L.Thiele. SPEA2: Improving the Strength Pareto Evolutionary Algorithm for Multiobjective Optimization. in Proc.Evolutionary Methods for Design, Optimisation, and Control. 2002. Barcelona, Spain.

10. K.Deb, et al. A Fast Elitist Multi-Objective Genetic Algorithm: NSGA-II. in Proceedings of the Parallel Problem Solving from Nature VI Conference. 2000.

11. N.Srinivas and K.Deb, Multiobjective optimization using nondominated sorting in genetic algorithms. Evolutionary Computation, 1994. 2(3): p. 221-248.

12. E.Zitzler and L.Thiele, Multiobjective Evolutionary Algorithms: A Comparative Case Study and the Strength Pareto Approach. IEEE Transactions on Evolutionary Computation, 1999. 3(4): p. 257-271. 
13. E. Zitzler, M.L., and L. Thiele. SPEA2: Improving the Strength Pareto Evolutionary Algorithm for Multiobjective Optimization. in In Proc.Evolutionary Methods for Design, Optimisation, and Control. 2002. Barcelona, Spain.

14. A.M.Anile, et al. Comparison among Evolutionary Algorithms and Classical Optimization Methods for Circuit Design Problems. in 2005 IEEE Congress on Evolutionary Computation (CEC'2005). 2005. Edinburgh, Scotland.

15. M.Jensen, Reducing the Run-Time Complexity of Multiobjective EAs: The NSGAII and Other Algorithms,. IEEE Transactions on Evolutionary Computation, 2003. 7(5): p. 503-515.

16. A.Tiwari, K.Vergidis, and B.Majeed. Evolutionary Multi-objective Optimization of Business Processes. in Evolutionary Computation, 2006. CEC 2006. 2006. Vancouver, BC, Canada.

17. L.T.Bui, et al. Performance analysis of evolutionary multi-objective optimization algorithms in noisy environm. in The 8th Asia Pacific Symposium on Intelligent and Evolutionary Systems. 2004.

18. D.Kuang and J.Zheng. Strategies Based on Polar Coordinates to Keep Diversity in Multi-Objective Genetic Algorithm,. in IEEE Congress on Evolutionary Computation. 2005. Edinburgh, Scotland.

19. H.Soh and M.Kirley. moPGA: Towards a new generation of multi-objective genetic algorithms. in IEEE congress on Evolutionary computation. 2006. Vancouver, BC, Canada.

20. J.Rumbaugh, I.Jacobson, and G.Booch, The unified modeling language user guide. 2005: Addison Wesley.

21. B.Bruegge and A.Dutoit, Object-Oriented software engineering using $U M L$, patterns, and Java. Second Edition ed. 2004: Prentice Hall.

22. R.Binder, Testing Object-Oriented Systems, models, patterns, and tools. 2000: Addison Wesley.

23. Binder, Testing Object-Oriented Systems, models, patterns, and tools. 2000: Addison Wesley.

24. J.D.Schaffer. Multiple objective optimization with vector evaluated genetic algorithms. in Proceedings of the First International Conference on Genetic Algorithms. 1985.

25. T.S.Chow, Testing software design modelled by finite state machines. IEEE Transactions on Software Engineering, 1978. 4(3): p. 178 - 187. 
26. S.Fujiwara, et al., Test Selection Based on Finite State Models IEEE Transactions on Software Engineering, 1991. 17: p. 591--603.

27. L.C. Briand, Y.L., Q. Lin. Improving State-Based Coverage Criteria Using Data Flow Information. in Proc. of IEEE International Conference on Software Reliability Engineering. 2005. Chicago, USA,.

28. B.Y.Tsai, S.Stobart, and N.Parrington. Employing data flow testing on objectoriented classes. in Software, IEE Proceedings-[see also Software Engineering, IEE Proceedings]. 2001.

29. N.Tracey, et al. An automated framework for structural test-data generation. in Proceedings of Automated Software Engineering, 1998. 13th IEEE International Conference 1998.

30. S.Kirkpatrick, C.D.Gelatt, and M.P.Vecchi, Optimization by Simulated Annealing. Science, 1983. 220(4598): p. 671-680.

31. N.Mansour and M.Salame, Data Generation for Path Testing. Software Quality Journal, 2004. 12(2): p. 121-136.

32. K.Derderian, et al. Generating feasible input sequences for extended finite state machines (EFSMs) using genetic algorithms. in Proceedings of the 2005 conference on Genetic and evolutionary computation. 2005.

33. Q.Guo, et al. Constructing multiple unique input/output sequences using metaheuristic optimisation techniques. in Software, IEE Proceedings-[see also Software Engineering, IEE Proceedings]. 2005.

34. K.Lakhotia, M.Harman, and P.McMinn. A multi-objective approach to searchbased test data generation. in Proceedings of the 9 th annual conference on Genetic and evolutionary computation. 2007.

35. J.R.Birt and R.Sitte. Optimizing testing efficiency with error-prone path identification and genetic algorithms. in Software Engineering Conference, 2004. Proceedings. 2004 Australian. 2004.

36. Z.Li, M.Harman, and R.M.Hierons, Search Algorithms for Regression Test Case Prioritization. IEEE Transactions on Software Engineering, 2007. 33(4): p. 225237.

37. L.C.Briand, J.Feng, and Y.Labiche, Experimenting with Genetic Algorithms and Coupling Measures to Devise Optimal Integration Test Orders. Software Engineering with Computational Intelligence, Kluwer, 2003.

38. G.Rothermel, R.H.Untch, and M.J.Harrold, Prioritizing test cases for regression testing. IEEE Transactions on Software Engineering, 2001. 27(10): p. 929-948. 
39. S.Elbaum, A.G.Malishevsky, and G.Rothermel, Test case prioritization: a family of empirical studies. Software Engineering, IEEE Transactions on, 2002. 28(2): p. 159-182.

40. H.Srikanth, L.Williams, and J.Osborne, System test case prioritization of new and regression test cases. Empirical Software Engineering, 2005. 2005 International Symposium on, 2005: p. 62-71.

41. K.Meffert, J.Meseguer, and E.D.Mart. Java Genetic Algorithms Package. 2005 [cited; Available from: http://sourceforge.net/projects/jgap.

42. J.J.Grefenstette, Optimization of control parameters for genetic algorithms. IEEE Transactions on Systems, Man and Cybernetics, 1986. 16(1): p. 122-128.

43. R.P.Pargas, M.J.Harrold, and R.Peck, Test-Data Generation Using Genetic Algorithms. Software Testing, Verification \& Reliability, 1999. 9(4): p. 263-282.

44. M.Laumanns, E.Zitzler, and L.Thiele. On the effects of archiving, elitism, and density based selection in evolutionary multi-objective optimization. in Proceedings of Evolutionary Multi-criterion Optimization (EMO 2001), Lecture Notes in Computer Science. 2001: Springer.

45. F.Rivas-Davalos and M.R.Irving. An Approach Based on the Strength Pareto Evolutionary Algorithm 2 for Power Distribution System Planning. in Evolutionary Multi-Criterion Optimization. Third Inter-national Conference, EMO. 2005: Springer.

46. Randy.Haupt, S.H., Practical Genetic Algorithms. 1998, New York: A WileyInterscience Publication.

47. M.Bowman, L.C.Briand, and Y.Labiche. Multi-Objective Genetic Algorithm to Support Class Responsibility Assignment. in Software Maintenance, 2007. ICSM 2007. IEEE International Conference on. 2007.

48. M.Bowman, Multi-Objective Genetic Algorithms to Support Class Responsibility Assignment, in System and Computer Engineering. 2007, Carleton University: Ottawa, ON, CA.

49. S.Elbaum, A.Malishevsky, and G.Rothermel. Incorporating varying test costs and fault severities into test case prioritization. in International Conference on Software Engineering: Proceedings of the $23 \mathrm{rd}$ International Conference on Software Engineering: Toronto, Ontario, Canada. 2001.

50. M.Hutchins, et al. Experiments of the effectiveness of dataflow-and controlflowbased test adequacy criteria. in Proceedings of the 16th international conference on Software engineering. 1994. 
51. Y.Ma, J.Offutt, and Y.Kwon, MuJava : An Automated Class Mutation System,. Software Testing, Verification and Reliability, 2005. 15(2): p. 97-133.

52. J.H.Andrews, et al., Using Mutation Analysis for Assessing and Comparing Testing Coverage Criteria. IEEE Transactions on Software Engineering, 2006. 32(8): p. 608-624.

53. K.Deb and D.Goldberg, An Investigation of Niche and Species Formation in Genetic Function Optimization, in Proceedings of the 3rd International Conference on Genetic Algorithms 1989. p. 42-50.

54. D.Whitley and N.Yoo, Modeling simple genetic algorithms for permutation problems, in Foundations of Genetic Algorithms 1994. p. 163-184

55. H.Kargupta, K.Deb, and D.Goldberg. Ordering genetic algorithms and deception. in Parallel Problem Solving from Nature PPSN II. 1992.

56. J.Magee and J.Kramer, Concurrency, State Models \& Java Programs, . 1999: John Wiley\&Sons. 


\section{Appendix A Multi-objective Genetic Algorithms}

\section{A.1 NSGA2}

The NSGA2 algorithm [10] is given as follows:

Step 1: Randomly generate the initial population $P_{0}$ of size $N$; assign a fitness value to each individual according to its rank, which is obtained by non-domination sorting of this population $P_{0}$ (the solutions in the same non-dominated frontier have the same fitness value); the usual binary tournament selection is used to generate the first offspring generation $Q_{0}$ of size $N$.

Step 2: Combine $P_{t}, Q_{t}$ to form a large population $R_{t}$ of size $2 N$; sort the whole large population $R_{t}$ and put all individuals at frontier 1 to $i(i>=1)$ into the next parent generation $P_{t+1}$. The individuals from frontier 1 to $i$ almost fill the population size $\mathrm{N}$ of $P_{t+1}$. A formal expression for this constraint is $\sum_{j=1}^{j=i}\left|F_{j}\right| \leq N \wedge \sum_{j=1}^{j=i+1}\left|F_{j}\right|>N$ where $\left|F_{j}\right|$ equals the number of individuals in frontier $j$. If $P_{t+1}$ is not filled by individuals from frontier 1 to $i$ $\left(\sum_{j=1}^{j=i}\left|F_{j}\right|<N\right)$, select $N-\sum_{j=1}^{j=i}\left|F_{j}\right|$ individuals from frontier $i+1$ using a crowdedcomparison operator $\prec_{n}$ and put them into the next parent generation $P_{t+1}$ to fill the population $P_{t+1}$. The crowded-comparison operator $\prec_{n}$ guides the selection process at the various stages and tends to select solutions that have less dominators and far from their neighbors in phenotype (big difference of the objective values with their neighbors).

Step 3: The new population $P_{t+1}$ of size $N$ is now used for selection, crossover and mutation to create a new population $Q_{t+1}$. Binary tournament selection is used, but the selection criterion is based on the crowded-comparison operator $\prec_{n}$. 
Step 4: Repeat step 2 to generate the next parent population $P_{t+2}$, and repeat step 3 to generate next offspring population $Q_{t+2}$ until the termination condition is true.

For all detail information about the algorithm, please refer to [10].

\section{A.2 SPEA2}

The SPEA2 algorithm [9] is given as follows:

Input: $N=$ regular population size; $\bar{N}=$ archive population size; $T=$ number of generations.

Step 1: Initialization phase: generate an initial population $P_{0}$ and create an empty archive population $\bar{P}_{0}=\phi$, time $t=0$.

Step 2: Fitness assignment: calculate the fitness values of individuals in $P_{t}$ and $\bar{P}_{t}$. For each individual $i$, its fitness value is $F(i)=R(i)+D(i)$. In this formula, $R(i)=\sum_{j \in P_{t}+\bar{P}_{t} j>i} S(j)$ is the raw fitness value. $R(i)$ is determined by the strengths of its dominators in the archive and regular populations. The strength of individual $j$ is $S(j)=\left\{k \mid k \in P_{t}+\bar{P}_{t} \wedge j \succ k\right\}$, representing the number of individuals it dominates. SPEA2 adapts the $k$-th nearest neighbour method presented by Silverman (1986). For each individual $i$, the distances in objective space (phenotype), to other individuals in the archive and regular populations, are calculated and sorted in a list in increasing order. The $k$-th element gives the distance, denoted as $\sigma_{i}^{k} . D(i)=\frac{1}{\sigma_{i}^{k}+2}$ is the density value corresponding to individual $i$, in which $k$ equals to $\sqrt{N+\bar{N}}$.

Step 3: Copy all non-dominated individuals in $P_{t}$ and $\bar{P}_{t}$ to $\bar{P}_{t+1}$. If the size of $\bar{P}_{t+1}$ is less than $\bar{N}$, then the best dominated individuals in $P_{t}$ and $\bar{P}_{t}$ are put into $\bar{P}_{t+1}$, which can be implemented by sorting the multi-set $P_{t}+\bar{P}_{t}$ according to the fitness value and copying 
the best ( $\bar{N}$ - size of $\left.\bar{P}_{t+1}\right)$ individuals $i$ with $F(i) \geq 1$ to $\bar{P}_{t+1}$; or if the size of $\bar{P}_{t+1}$ exceeds the archive population size $\bar{N}$, then the truncation operator is applied to reduce the size of $\bar{P}_{t+1}$ to $\bar{N}$, which can be done by removing individuals $i$ that satisfy the following condition:

$\left.\left.i \leq_{d} j: \Leftrightarrow \forall 0<k<\left|\bar{P}_{t+1}\right|: \sigma_{i}^{k}=\sigma_{j}^{k} \vee \exists 0<k<\left|\bar{P}_{t+1}\right|:\left[\forall 0<l<k: \sigma_{i}^{l}=\sigma_{j}^{l}\right) \wedge \sigma_{i}^{k}<\sigma_{j}^{k}\right]\right)$

It means that solution $i$ is dominated by solution $j$ if solution $i$ and solution $j$ have exactly same distances to all other solutions (they are redundant solutions), or if solution $i$ and solution $j$ have exactly same distances to $l$ solutions and the distance from solution $i$ to solution $k$ is shorter than the distance from solution $j$ to solution $k$.

Step 4: Mating selection: perform a binary tournament selection with replacement on $\bar{P}_{t+1}$ in order to fill the mating pool.

Step 5: Apply genetic operator crossover and mutation to generate the regular population $P_{t+1}$. Increase generation to $t=t+1$, and go to step 2 until $T$ is reached.

For all detail information about the algorithm, please refer to [9]. 


\section{Appendix B The method to Identify All Definitions or All DU Pairs Covered by a Transition Sequence}

1. The operation contracts is manually written in java code. The usage (definition, $\mathrm{C}$-use or P-use) of each data element is be identified manually, and a statement describing the usage of data flow element, including attribute name, usage type, transition id, the term of operation contract, etc., is added immediately before the usage.

2. Create a two dimension array for each attribute in the state machine diagram.

3. Execute the java code with a given transition sequence: if a definition of an attribute is encountered, add a definition with the usage description into the array; if a use of an attribute is encountered, add a use with the usage desription into the array.

4. After all transitions in the transition sequence are executed, collect all definition and use information for each attribute. Then we obtain the following array:

\begin{tabular}{|c|c|c|c|c|c|}
\hline Def & P-use & C-use & Def & C-use & $\ldots$ \\
\hline t1_Term2 & t2_Term1 & t2_Term5 & t2_Term9 & t5_Term2 & $\ldots$ \\
\hline
\end{tabular}

5. From this array, we can get all definitions, all P-use, all C-use and all DU pairs information that the transition sequence has covered. 


\section{Appendix C Input File for GA}

// TotalCasenumber defines how many test cases in a test suite.

TotalCaseNumber $=20$

$1 /$ TotalTransitions defines how many transitions in a test suite. TotalTransitions $=72$

// MaxDef defines how many data flow information a test suite covers

MaxDef $=90$

// the user specified data flow coverage rate, default value is 1.0

Specific_CoverageRate $=1.0$

$/ /$ the user specified test cost length default value equals to the

// number of TotalTransitions

Specific_CostLength $=72$

// the following three fields are used to decide how to modify SC and $T$

// how to decide the plateau, for example, the average data flow

$/ /$ coverage rate in archive set does not have $2 \%$ change within 50

// generations, then the evolution reaches a plateau.

Plateau_generation $=50$

Plateau_percentage $=2$

Specific_CostLength_Modifiable_Thread $=90$

// The following fields define the GA parameters.

ga. NumberOfGenerations $=500$

ga. SelectionRate $=0.9$

ga. TournamentSize $=2$

ga. Populationsize $=100$

ga. Archivesize $=50$

ga. CrossoverRate $=0.7$

ga. MutationRate $=100$

$/ /$ Test cases - changing according to criterion

$/ / \mathrm{TC} 1=t 1, t 3$ means test case 1 contains two transitions $t 1$ and $t 3$ in

$/ /$ a sequence.

$/ / \mathrm{TC1t3}=$ state def_t1_engineon_Term5 puse_t3_accelerate_Term1,... means 
// in the test case 1 transition 3 what kind data flow information is

// contained. The above data flow information means the variable

$1 /$ "state" has a definition in transition t1 method engineon at Term 5

$/ /$ and a use in transition $t 3$ method accelerate at Term1. So this is

// a DU pair.

$\mathrm{TC} 1=\mathrm{t} 1, \mathrm{t} 3$,

$\operatorname{TC} 1 \mathrm{t} 1=$

TC1t3 = state def_t1_engineOn_Term5 puse_t3_accelerate_Term1, state def_t1_engineon_Term5

def_tl_engineon_Term3

def_t1_engineon_Term3

def_t3_accelerate_term6

def $t 1^{-}$engineon Térm2

def $t I^{-}$engineon Term2

def $t 1$-engineon_Term2

def_t1_engineon_term2

def_t1_engineon_Term4 puse_t3_accelerate_Term2, puse t3_accelerate_Term10, throttle pusé_t $\overline{3}$ acceleraté Term5, throttle cuse $t 3$ accelerate Term6, throttle puse t 3 accelerate Term9, speed puse_t3_accelerate_Term8, speed puse t3 accelerate Term9, speed cuse $\bar{t} 3$ accelerate $\bar{T}$ erm10, speed cuse_t3_acceilerate_Term $\overline{1} 0$, brakepadel

$\ldots$

$\ldots$

$\mathrm{TC} 20=t 1, t 5, t 10, t 15$,

$\mathrm{TC} 20 \mathrm{t} 1=$

TC20t5 = state def_t1_engineon_Term5 puse_t5_on_Term8, cruisespeed def_t1_engineon_Term1 - - puse_t5_on_Term2, cruisespeed def_t1_engineon_Term1 puse_t5_on_Term5, speed ${ }^{-}$def_t1_engineon_Term2 puse $-t \overline{5}$ _on_Term $\overline{2}$,

TC20t10 = state def_t5_on_Term9 puse_t10_brake_Term13, throttle def_t1_engineon_Term3 puse_tio_brake_Term2, speed de $\bar{f}_{-} t 1$ _engineon_Term2

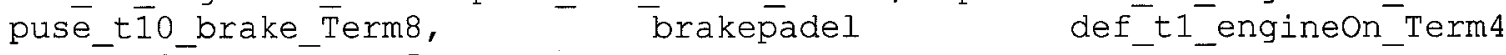
puse_t10_brake_Term5,

TC20t15 = state def t10 brake Term14 puse t15 engineoff Term1, cruisespeed def_t1_engineōn_Tërm1 puse_t15_engineoff_Term3, throttle def_t1_engineon_Term3 - puse_t15_engineoff_Term $\overline{9}, \quad$ speed def_t1_engineon_Term2 puse_t15_engineoff_Term6, brakepadel def_t1o_brake_Têrm6 puse_t15_engiñeoff_tTerm12, 


\section{Appendix D Output File}

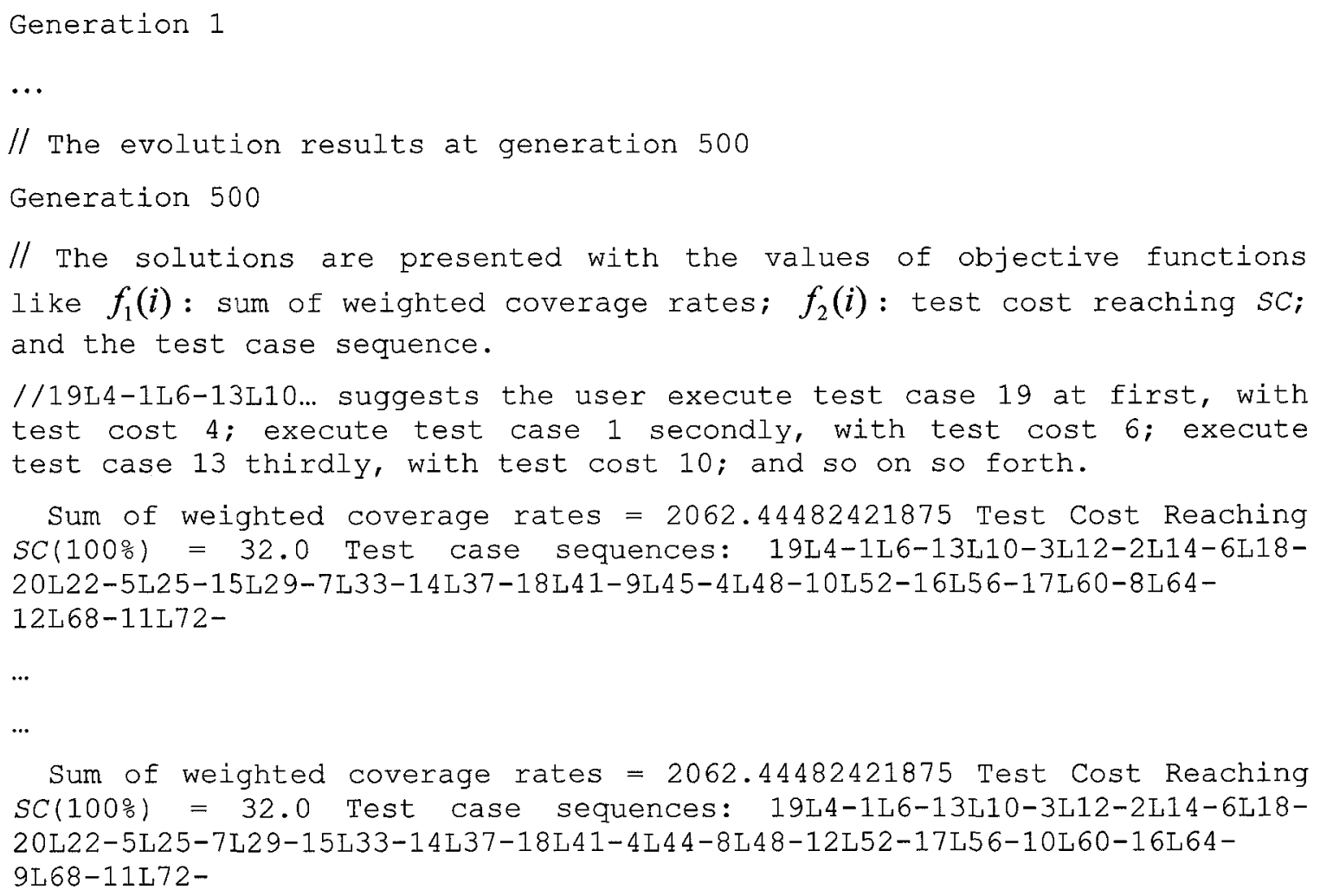




\section{Appendix E The Three Case Studies (System, Test Suite, Mutants)}

\section{E.1 Cruise Control System}

The Cruise Control System from Magee [56] is implemented in Java and contains six classes: CruiseControl, CruiseDisplay, CarSpeed, Controller, SpeedControl and Carsimulator. From its analysis model, a state machine diagram is derived, as shown in Figure 24; this is a rare example without any guard conditions. This state machine diagram indicates the state-based behavior of a cruise control object. But in its implementation, the behaviors are achieved by the cooperation of three objects: Controller, Carsimulator and speedControl.

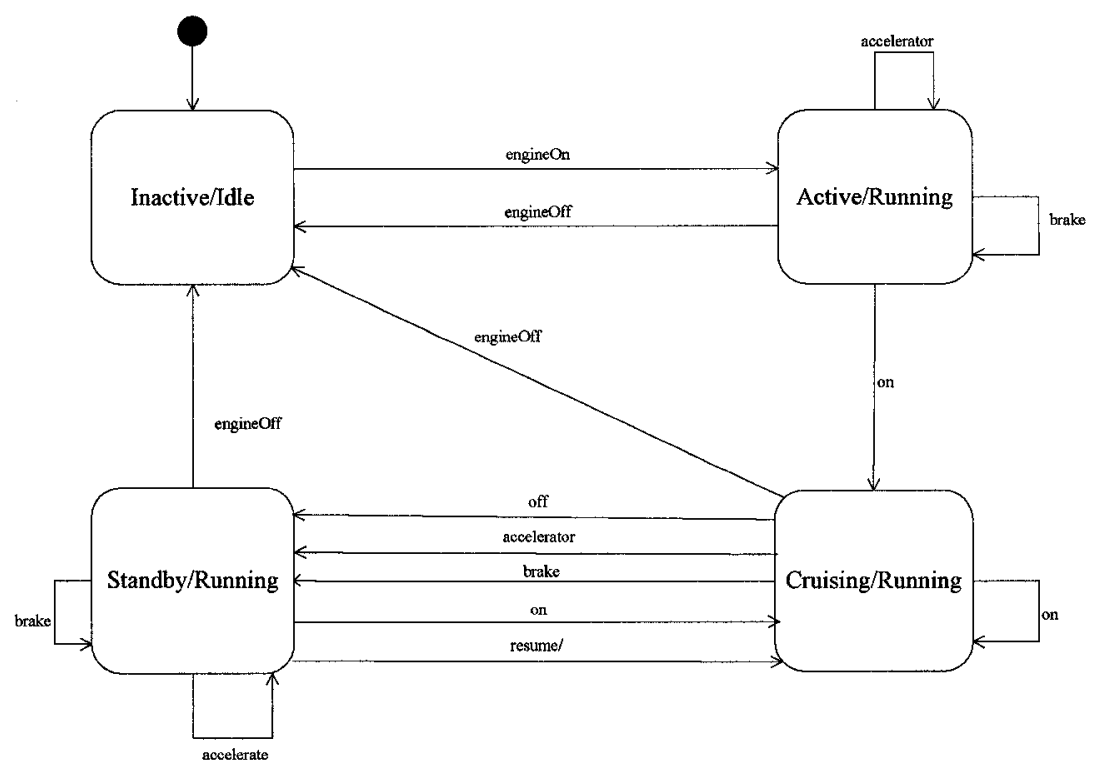

Figure 24: State machine diagram of the Cruise Control System

Following Briand et al.'s algorithm, we derive the transition tree of Figure 25, which contains 20 test cases. 


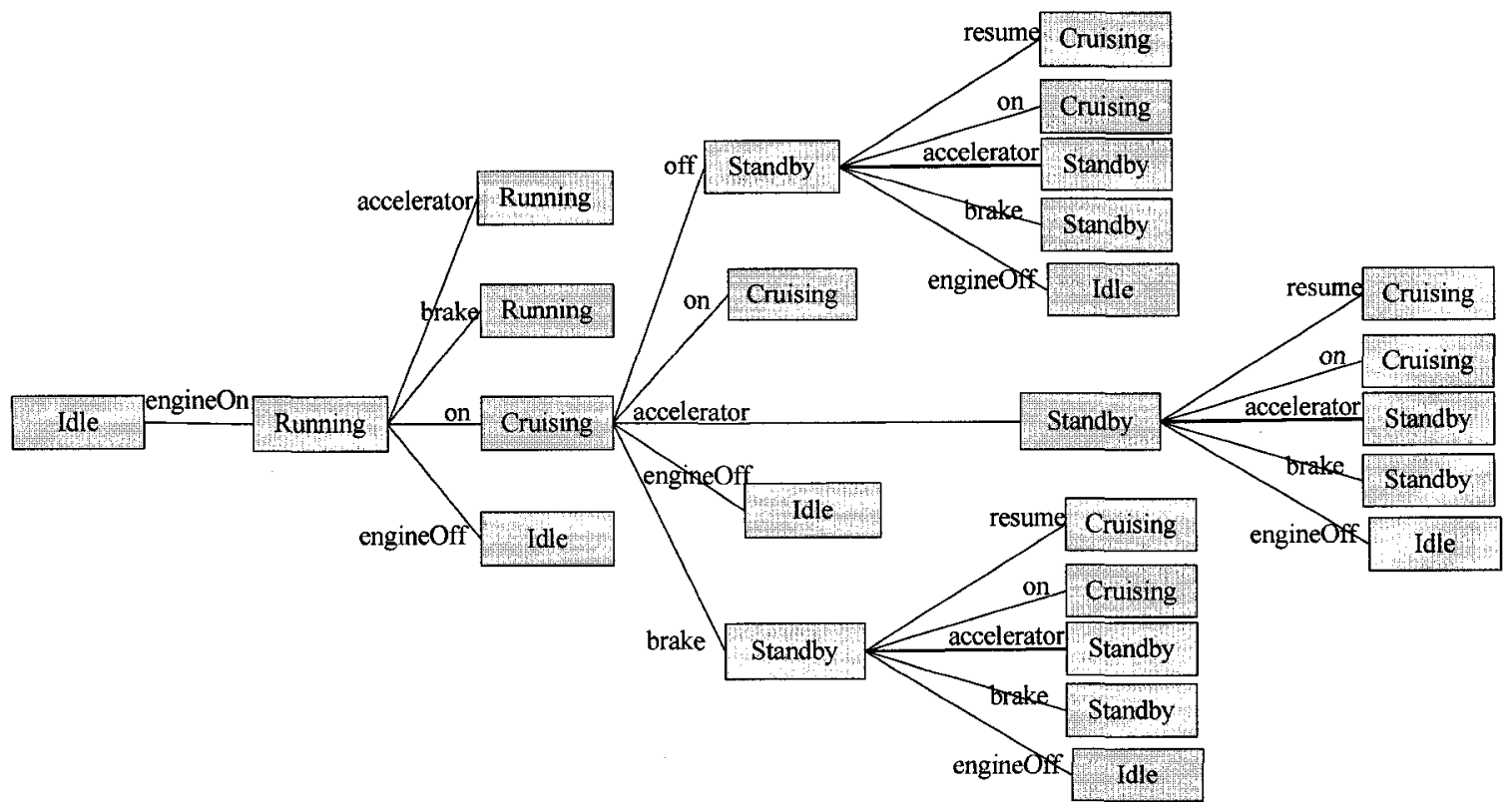

Figure 25: Modified transition tree for Cruise Control System

MuJava generates 494 mutants for the Cruise Control System, eight of which result in compilation errors. Of these, 350 mutants are detectable by the full test suite. Within the 136 undetectable mutants, 66 are equivalent mutants and 70 mutants are not detected because the oracle is not precise enough.

Figure 26 is an analysis of mutants killed by test cases: Y percent of the mutants are killed by $X$ percent of the test cases. When accounting for 350 mutants, a test case detects on average $48.7 \%$ of the mutants. This is much higher than $16 \%$ average detection probability as reported in [52]. Reaching a similar probability in our case study can only be achieved by removing so many mutants that we would end up with less than 100 mutants. To ensure we have enough mutants and our average detection probability is comparable with the one reported in [52], we removed the 116 mutants which can be detected by more than $70 \%$ of the test cases. The new relationship between killed mutants and test cases is shown in Figure 27. The new average detection probability is $27.4 \%$. According to [52], the mutants that are only detected by fewer than $1.5 \%$ test cases are considered "very hard", and the mutants that are detected by $1.5 \%$ to $5 \%$ test cases are considered "hard". $20.5 \%$ of the mutants are hard to detect and no mutant is very hard to detect. These 234 mutants are created from method-level operators AOIS, AOIU, AORB, 
ASRS, COI, COR, LOI, ROR and class-level operator JSI. Figure 28 shows the distribution of the 234 mutants per mutation operator.

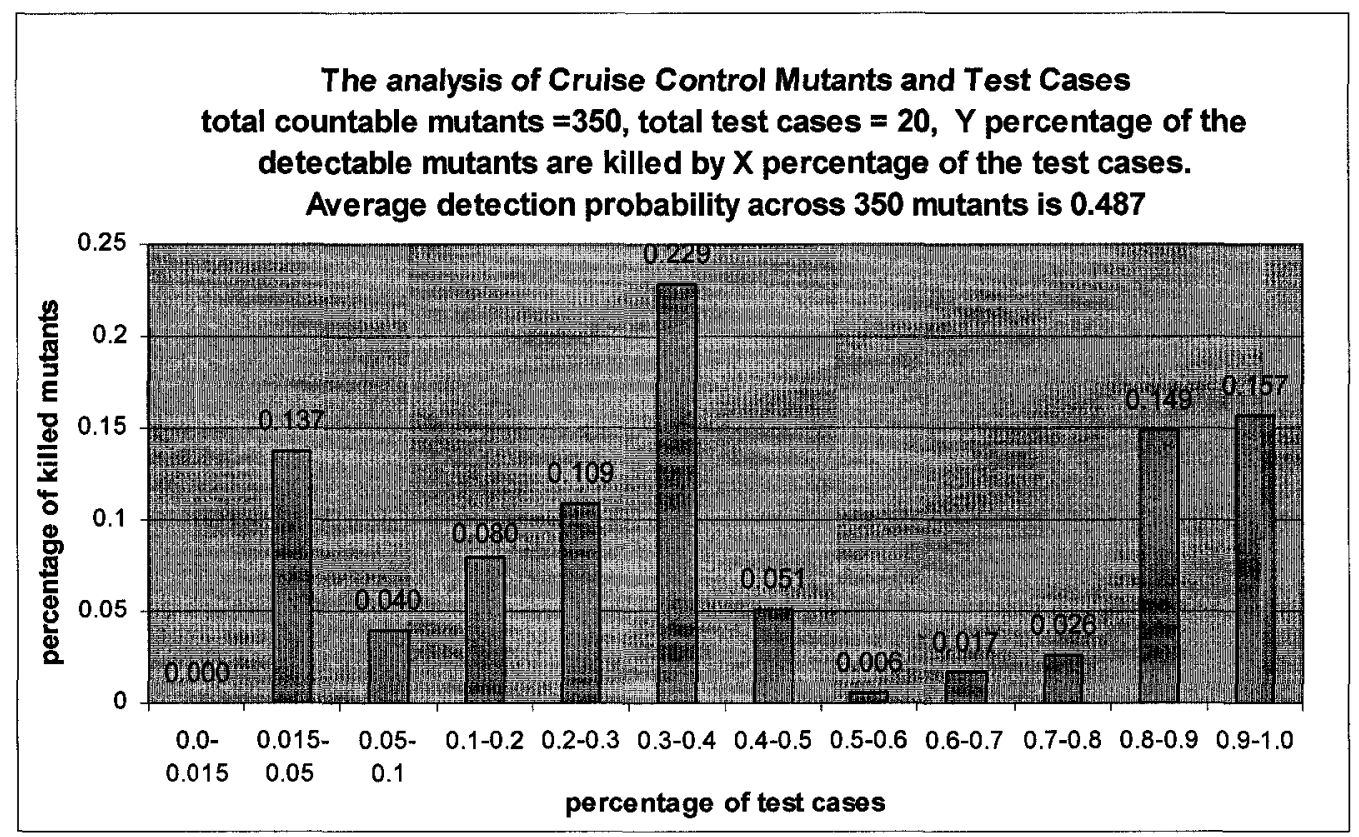

Figure 26: Analysis of 350 mutants killed by 20 test cases for Cruise Control System

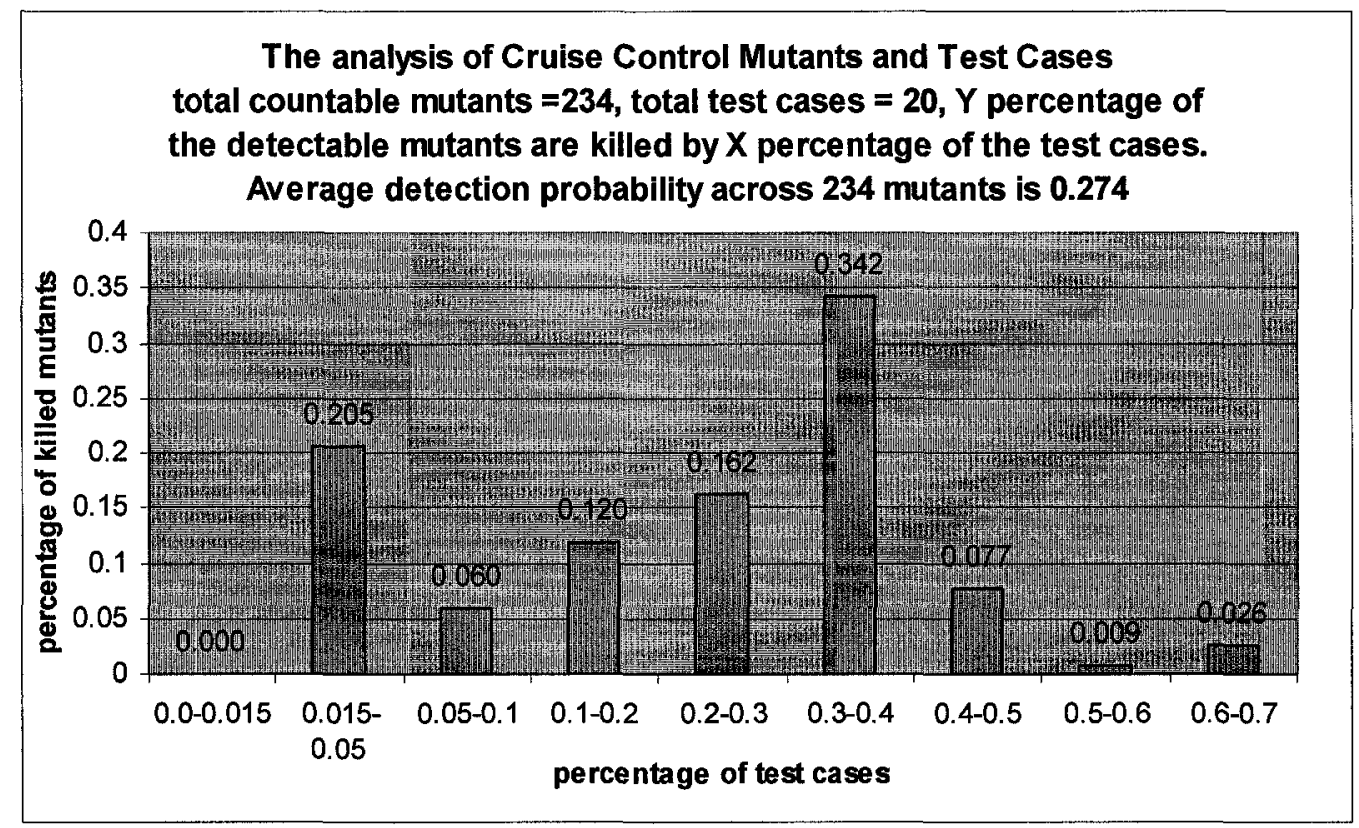

Figure 27: Analysis of 234 mutants killed by 20 test cases for Cruise Control System 


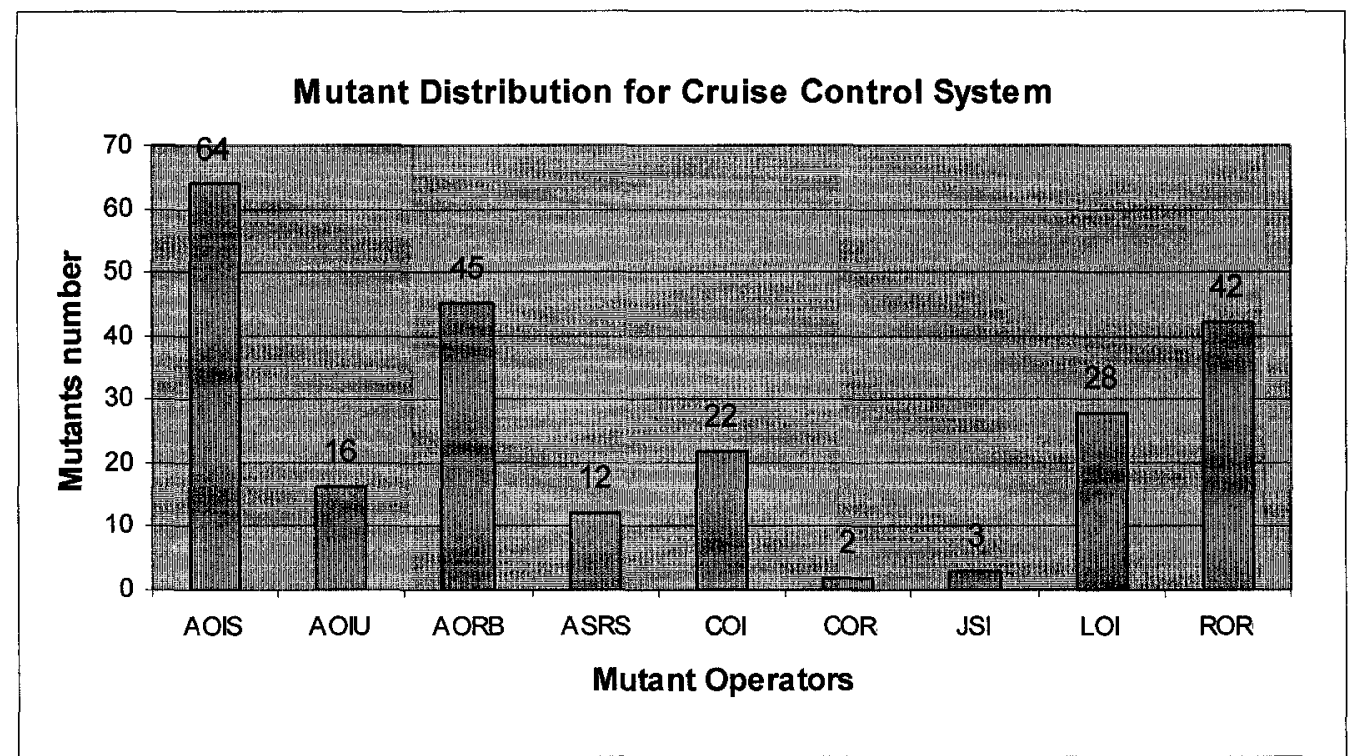

Figure 28: Mutant operator distribution for Cruise Control System

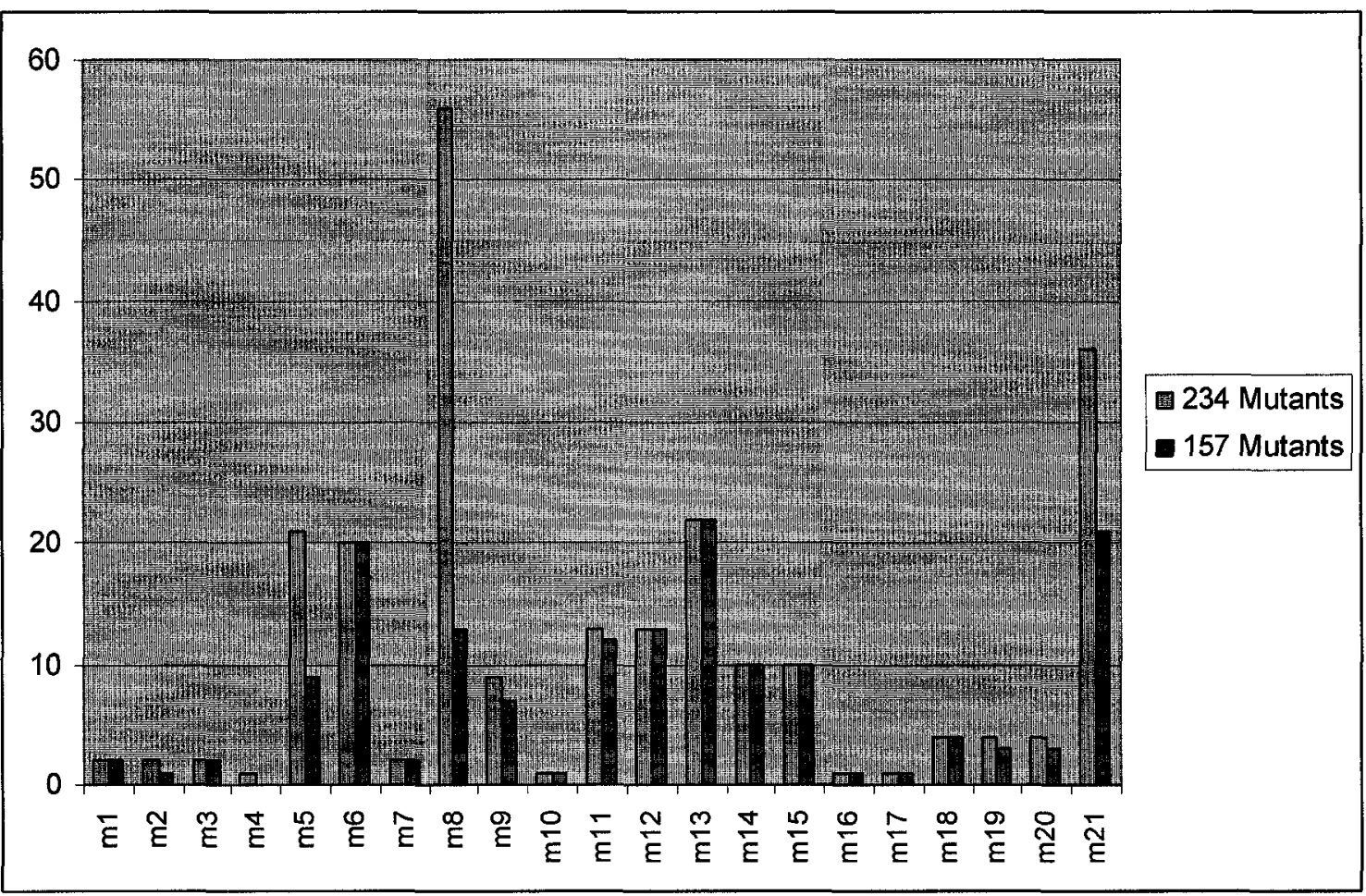

Figure 29: Mutant distribution per method in source code, before (234) and after (157) balancing 


\section{E.2 Ordered Set}

Class OrdSet represents a bounded, ordered set (no duplicate) of integers. The OrdSet has a resizing functionality that is triggered when it is full and the user wants to add elements anyway. (There is a limited number of resize operations allowed.) It is implemented in Java and contains two classes: OrderSet and OverFlowException.

Its state machine diagram is provided in Figure 30. It is interesting because almost all the transitions are guarded by one or two conditions. This greatly increases the difficulty of deriving a modified transition tree and the number of transcribed test cases. Also, based on a modified transition tree, the state "Overflow" is not reachable from the "Filled" state, although the behavior of a SUT object moving from "Filled" to "Overflow" should be quite interesting and important. We were highly interested in this and manually modified the transition tree and added the transitions that led the SUT object to move from "Filled" to "Overflow". The complete derived transition tree is large, with 165 terminal leaves (and a total of 916 transitions), that we present only a part of the derived transition tree in Figure 31. 


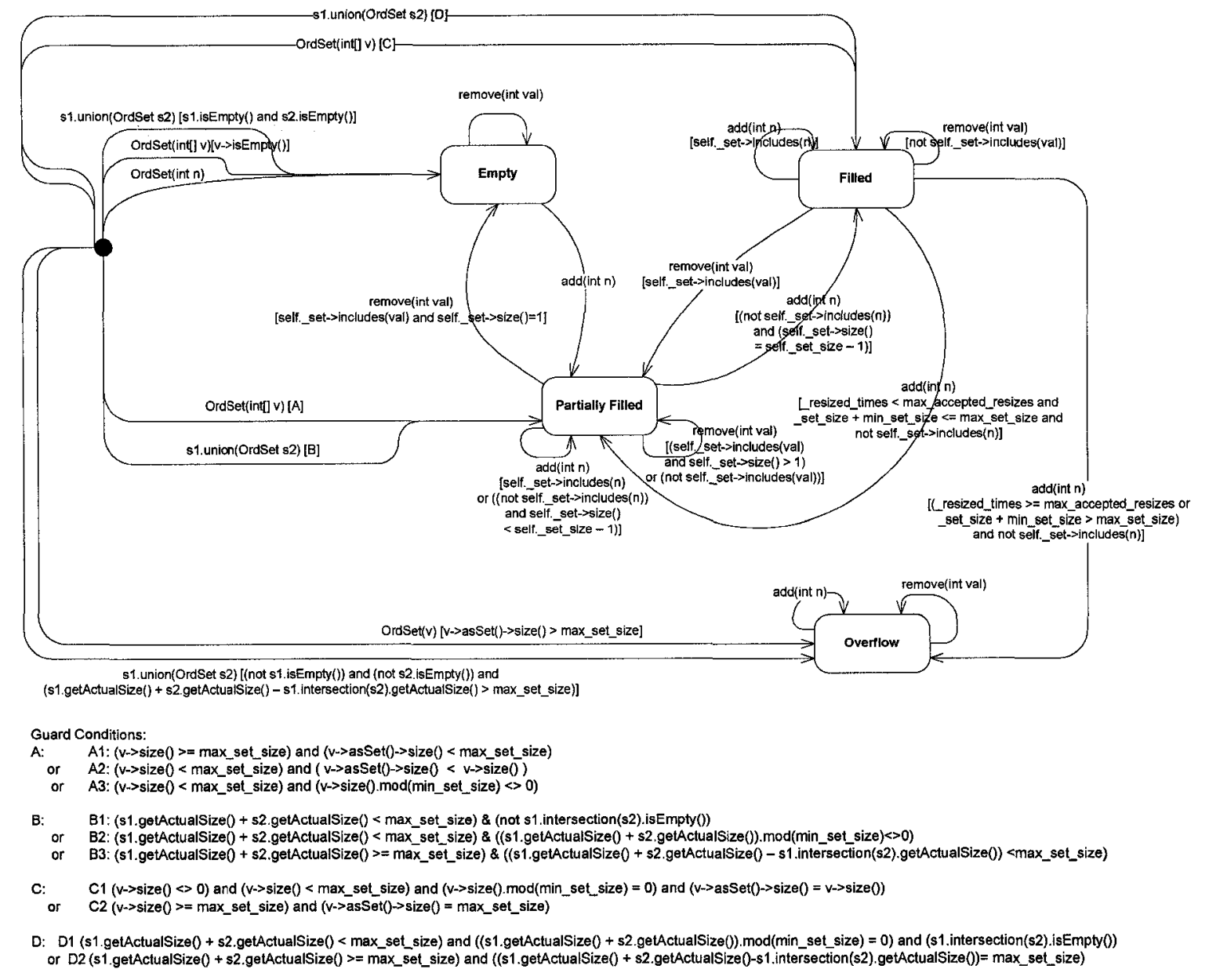

Figure 30: State machine diagram of Ordered Set 


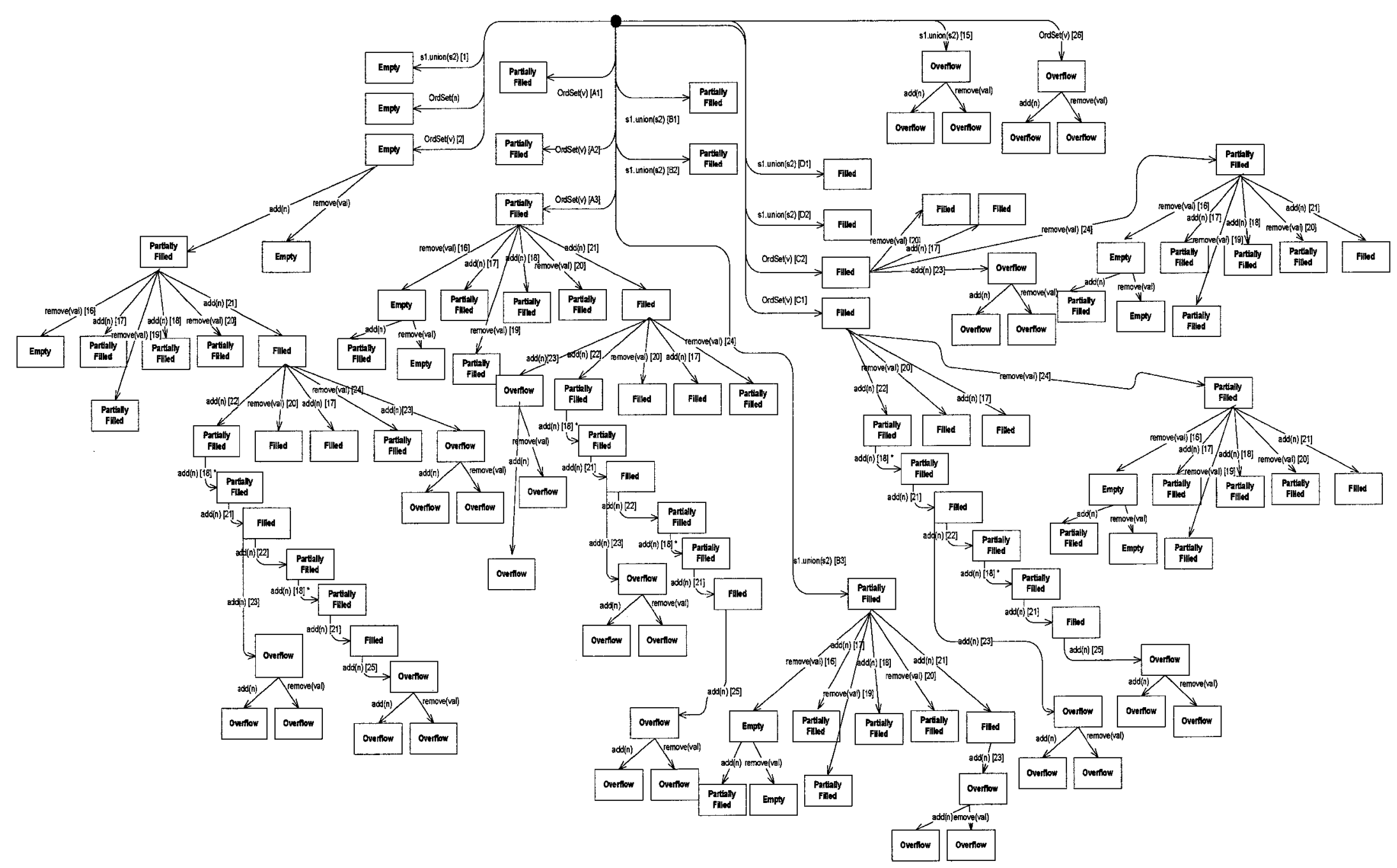

Figure 31: Incomplete transition tree for Ordered set 
One important issue should be clarified: the source code of Ordered Set includes several methods (contains (Ordset a), equals (Ordset a), isEmpty(), isoverflow(), elementAt (int $\mathrm{n}$ ) and tostring ()), which are never invoked by any test case derived from the modified transition tree. This is because we simplified the state machine diagram and omitted these methods from the model. Therefore, all mutants modifying the source code of these methods are not detectable.

MuJava generates 1,013 mutants for the Ordered Set system. 151 of those mutants are in those un-executed methods. There are 68 equivalent mutants. Test cases could not be executed on 33 mutants because of a compilation error. The analysis of 761 mutants killed by 165 test cases is shown in Figure 32 .

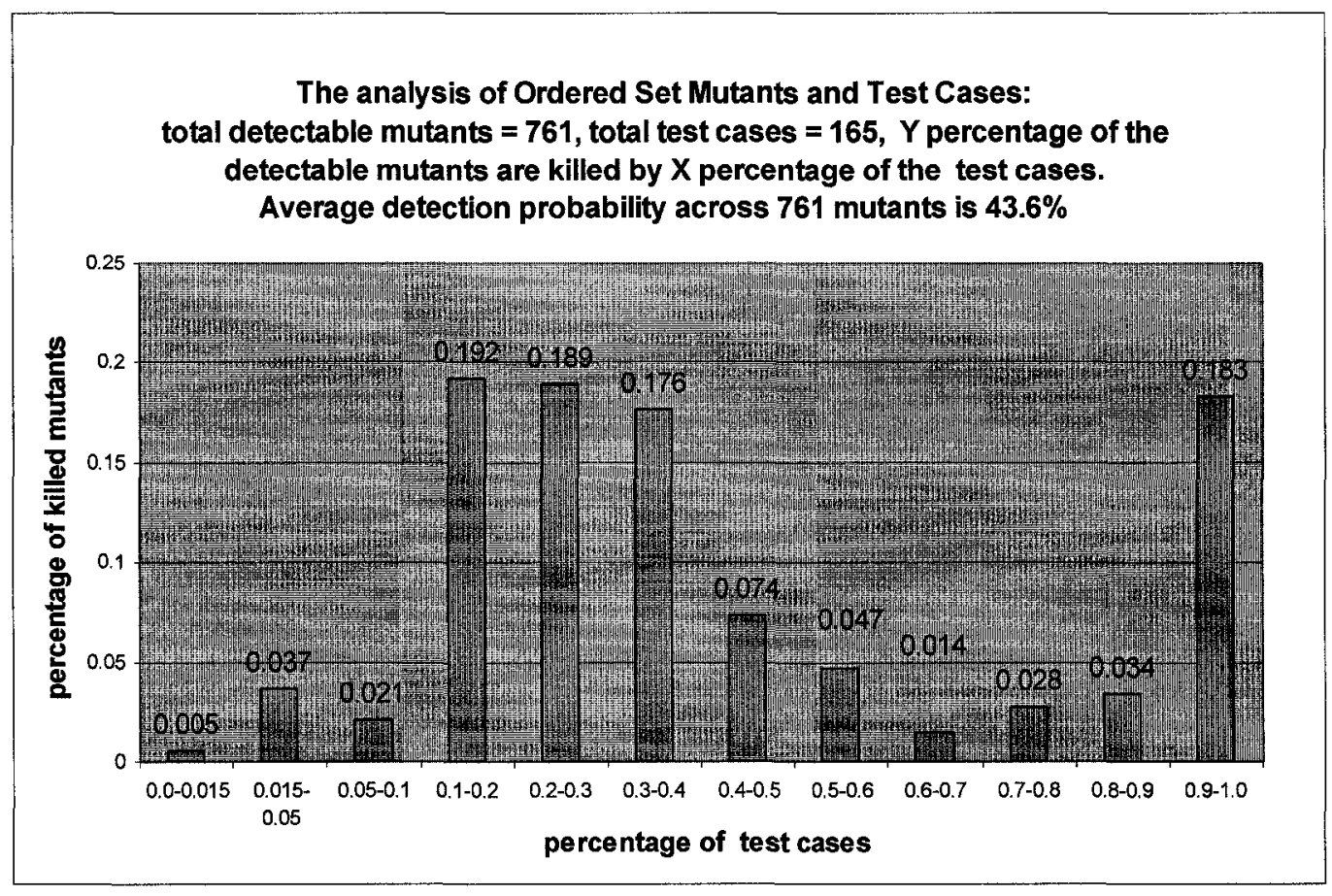

Figure 32: Analysis of 761 mutants killed by 165 test cases for Ordered Set

About $18.3 \%$ mutants will be killed by more than $90 \%$ of the test cases, and the average detection probability is $43.6 \%$. Referring to paper [52], which retains the average detection probability at $16 \%$, we decided to remove all mutants that could be detected by more than $70 \%$ of the test cases, thereby removing 186 mutants. 


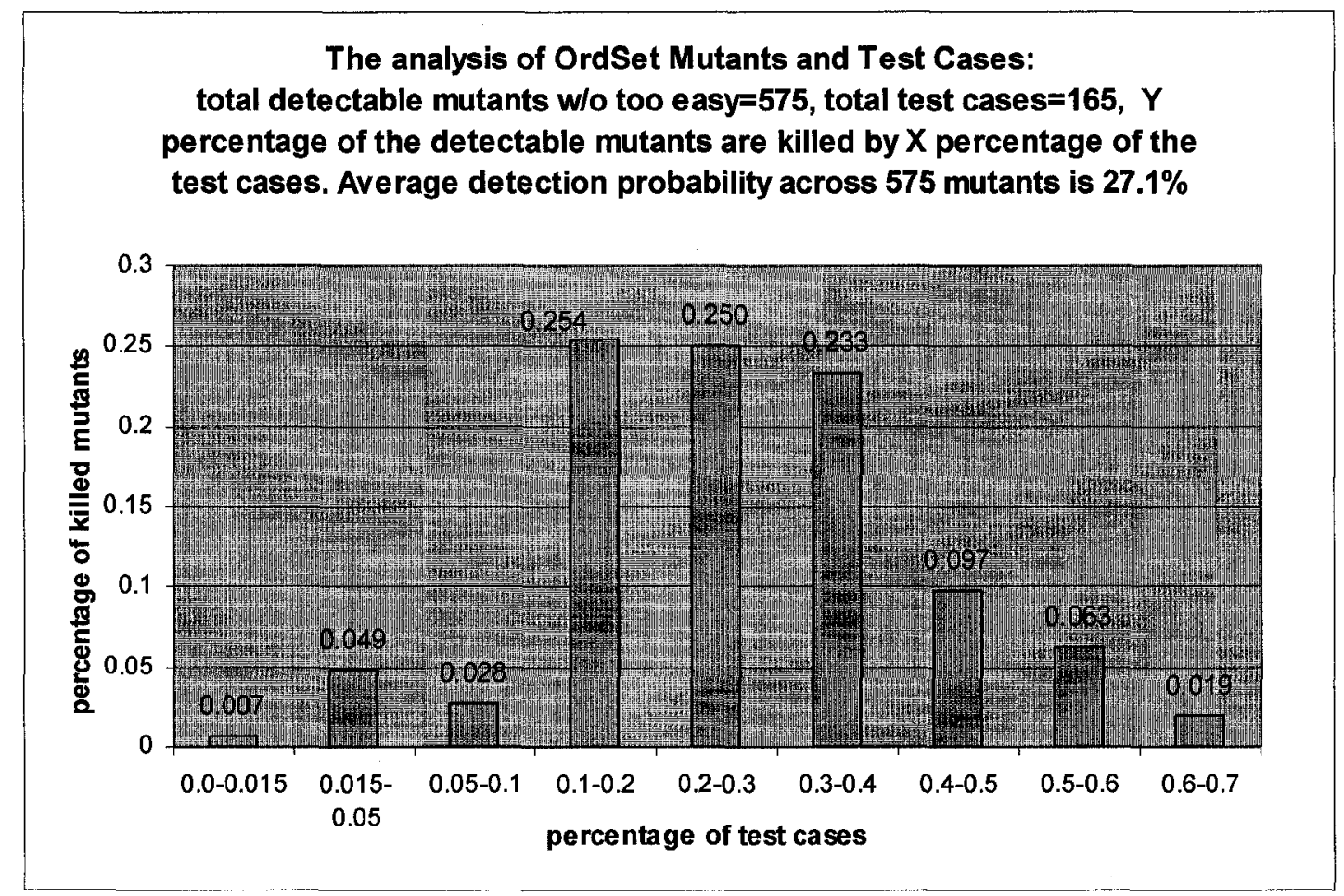

Figure 33: Analysis of 575 mutants killed by 165 test cases for Ordered Set

The new detection relationship of mutants and test cases is shown in Figure 33. The average detection capability is reduced to $27.1 \%$. About $4.9 \%$ mutants are hard to detect and $0.7 \%$ mutants are very hard to kill.

Of the 575 remaining mutants, the mutant operators include method-level operators AODU, AOIS, AOIU, AORB, AORS, COD, COI, COR, LOI, ROR and class-level operator EAM, JSI. Figure 34 lists the number of each mutant operator. 


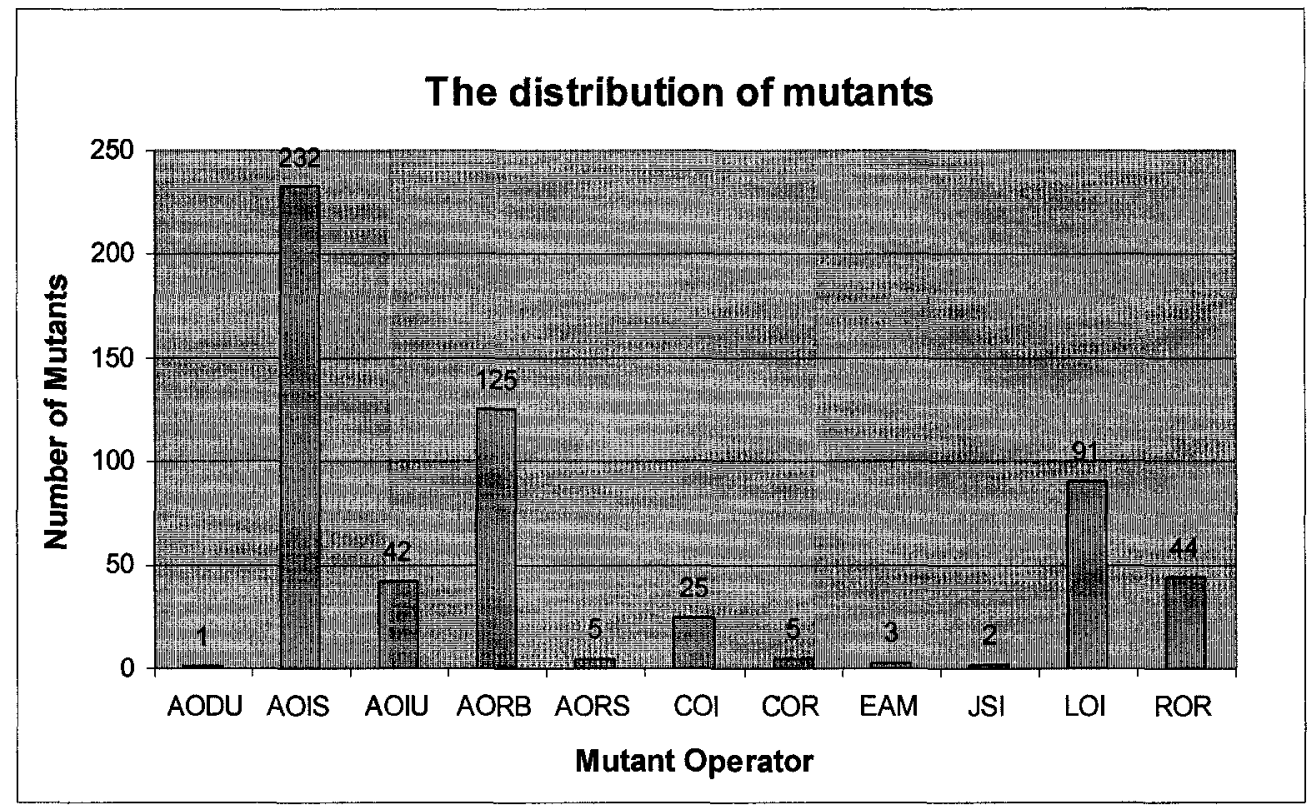

Figure 34: Mutant operator distribution for Ordered Set

\section{E.3 VCR}

A VCR system is implemented in Java using the state design pattern. It contains 29 classes, including 14 state classes and 11 event classes; one controller class Vcr; and three auxiliary classes. At the analysis level, one single class Vcrcontroller can be used to model the state-dependent behavior of the VCR: Figure 35. 


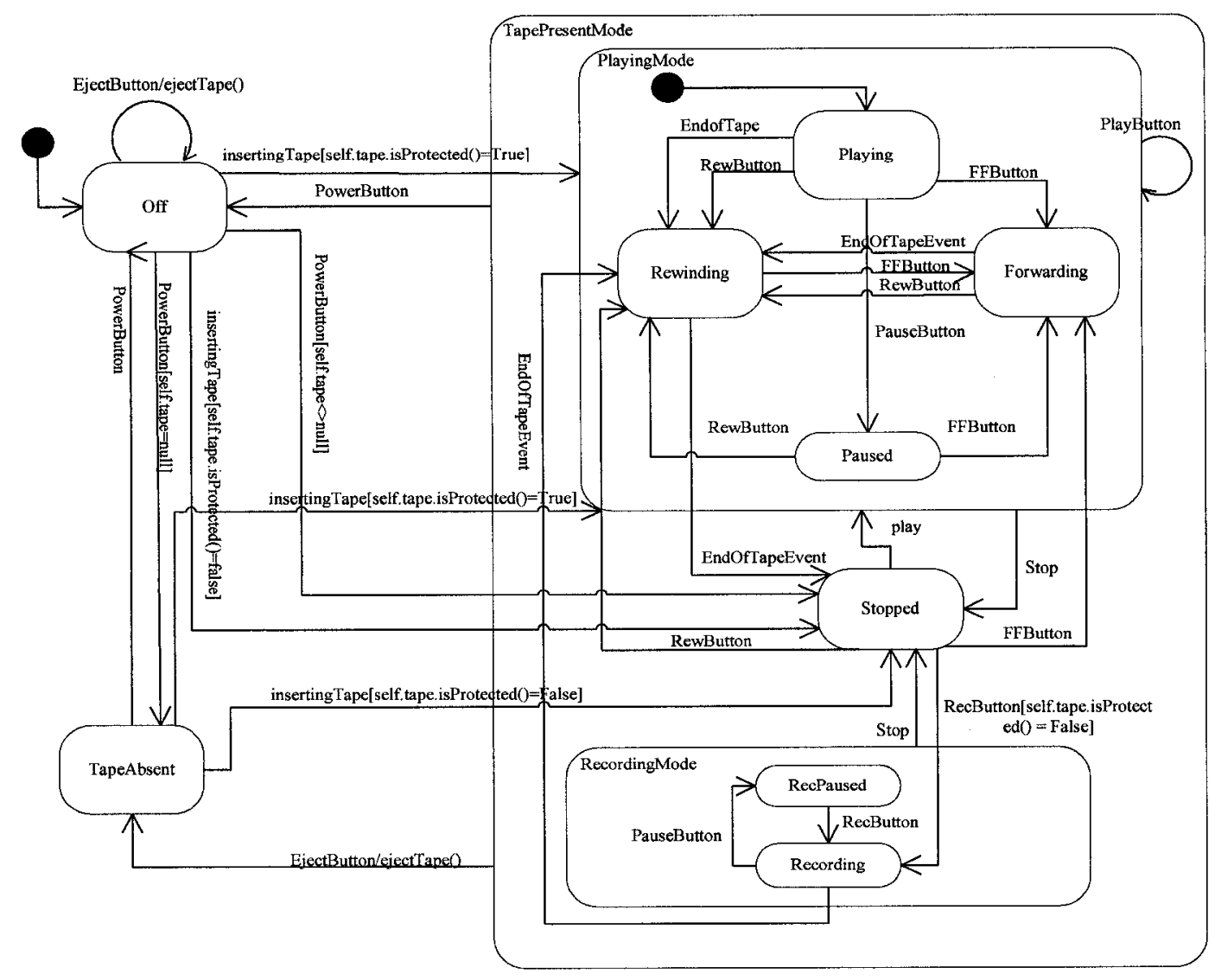

Figure 35: State machine diagram for VCR system

The number of test paths in the modified transition tree is 1,548 . Due to space limitations, we can not include the transition tree here, even an incomplete one. Because there are so few guard conditions in this state machine diagram, we generated these test paths automatically.

MuJava generates 488 mutants for the VCR system. Of these 488 mutants, 17 mutants cause a compilation error, 21 are equivalent mutants, and 450 mutants are detectable. Figure 36 below indicates the analysis of 450 mutants killed by 1548 test cases. 


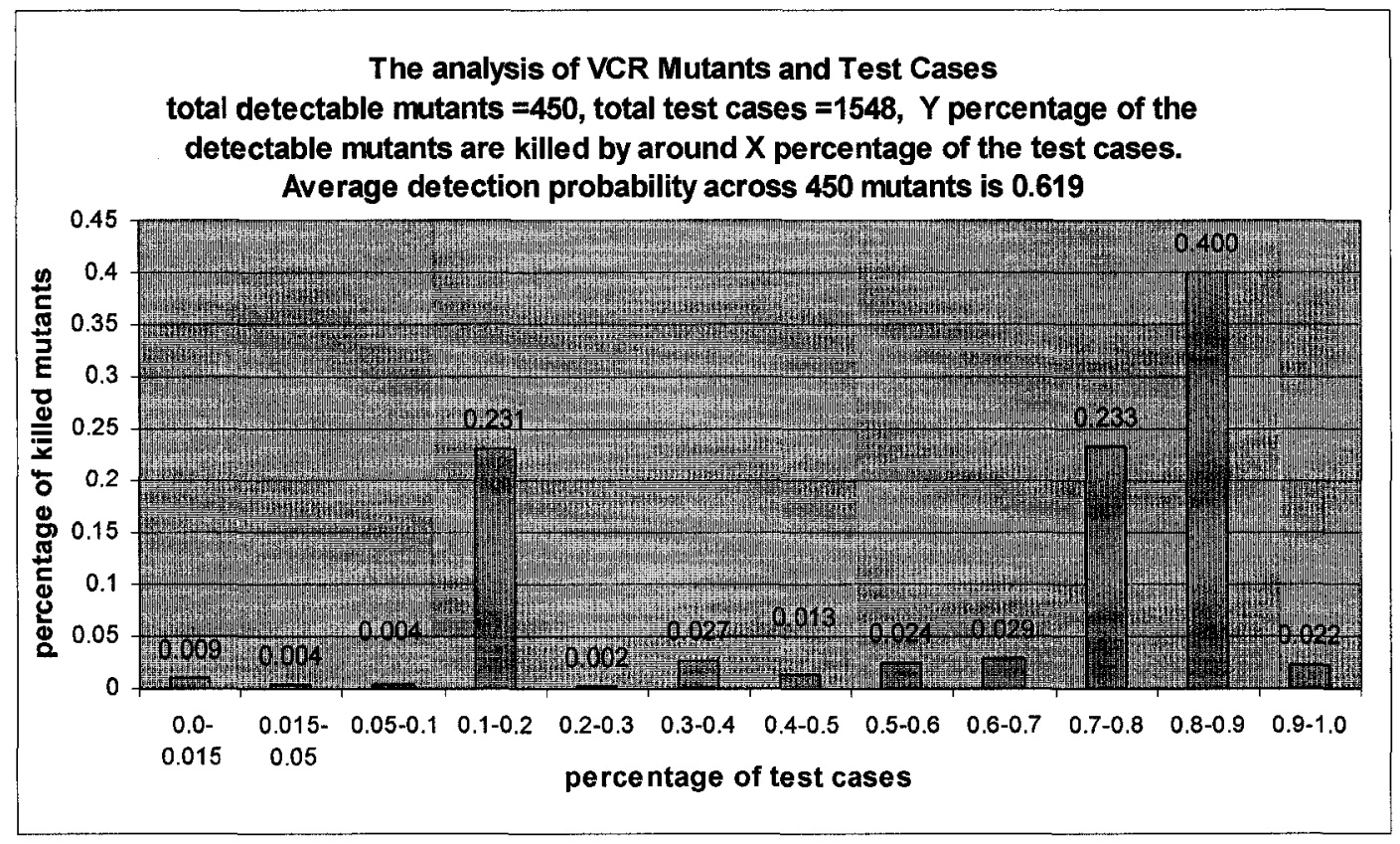

Figure 36: Analysis of $\mathbf{4 5 0}$ mutants killed by 1548 test cases for VCR System

The average detection capability is $61.9 \%$. It was too easy to detect 296 mutants for more than $70 \%$ of test cases. We removed all those "easy to kill" mutants. The mutant operators of the remaining 175 mutants are method-level operators, AOIS, AOIU, AORB, COD, COI, LOI, ROR and class-level operators, EAM, PCI, JSI. The new average detection capability is $22.1 \%$. Figure 37 shows the new mutant detection probability and Figure 38 shows the distribution of mutant operators. 1.3\% mutants are hard to detect and $2.6 \%$ mutants are very hard to kill. 


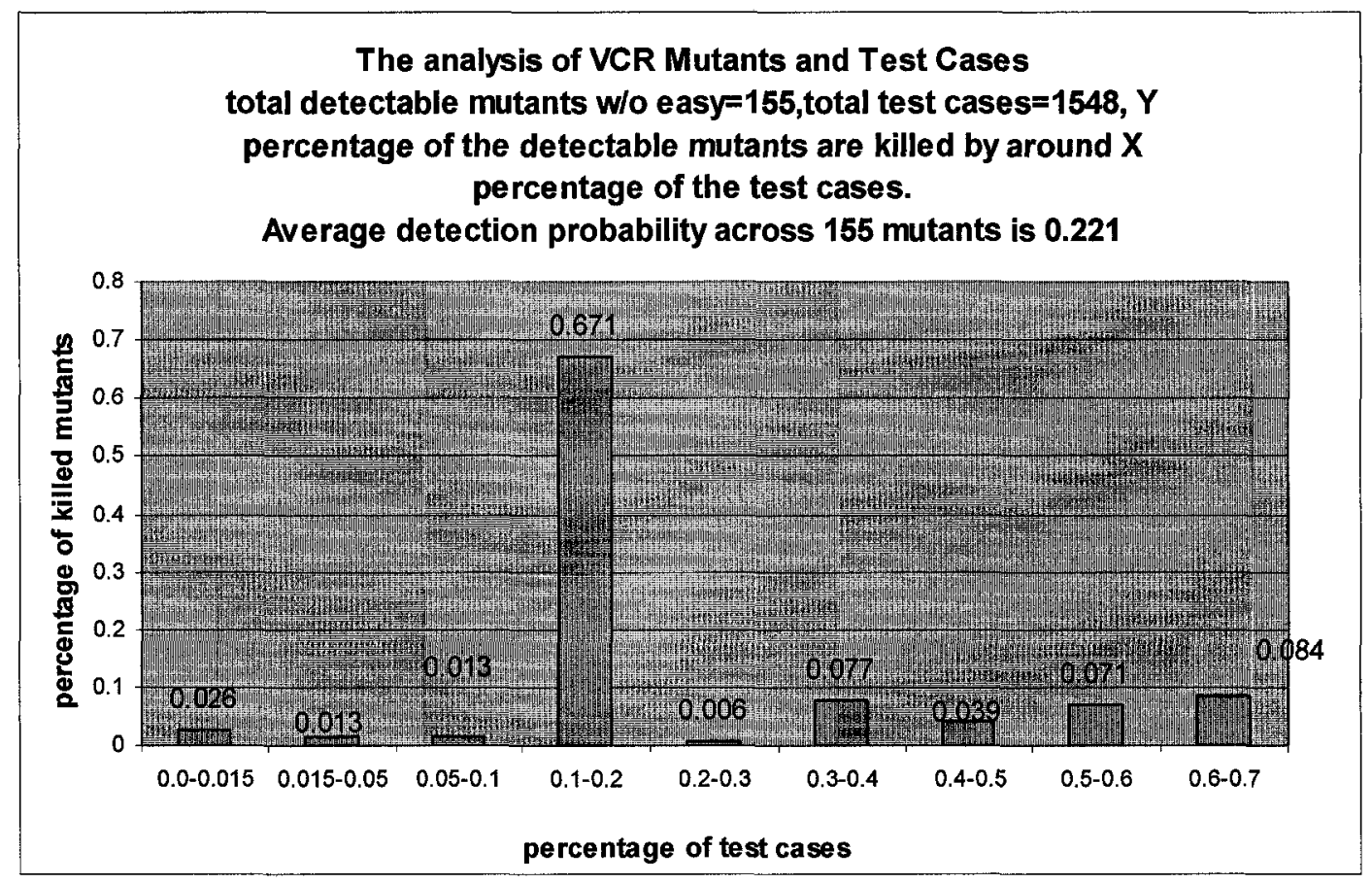

Figure 37: Analysis of 175 mutants killed by 1548 test cases for VCR system

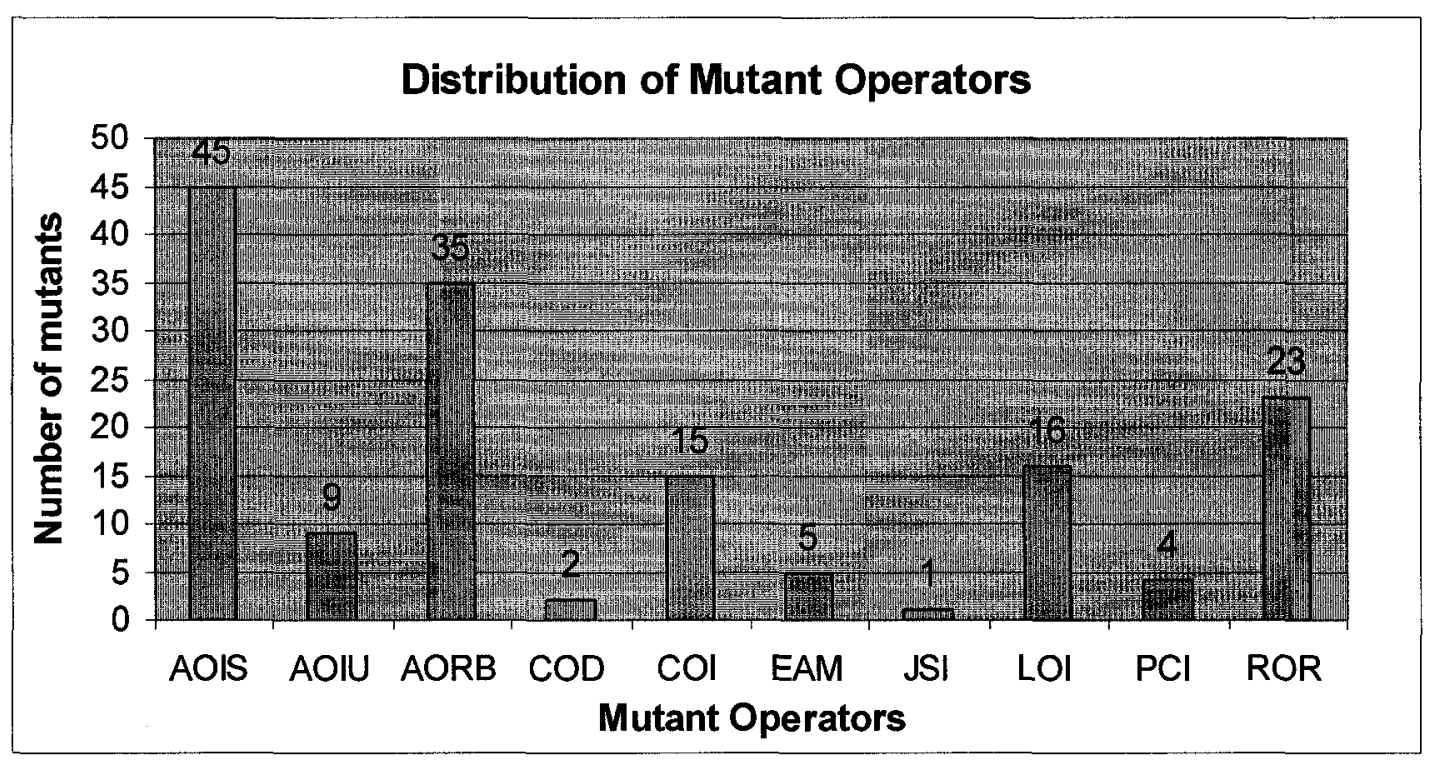

Figure 38: Mutant operator distribution for VCR System 


\section{Appendix F Operation Contracts for the Three Case Studies.}

\section{F.1 Operation contracts of the Cruise Control System}

Attributes:

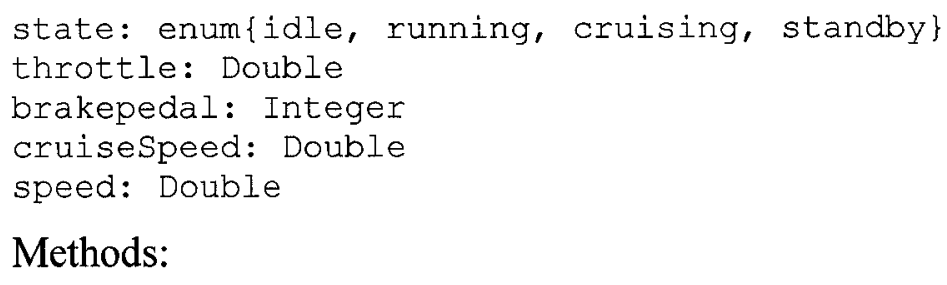

Methods:

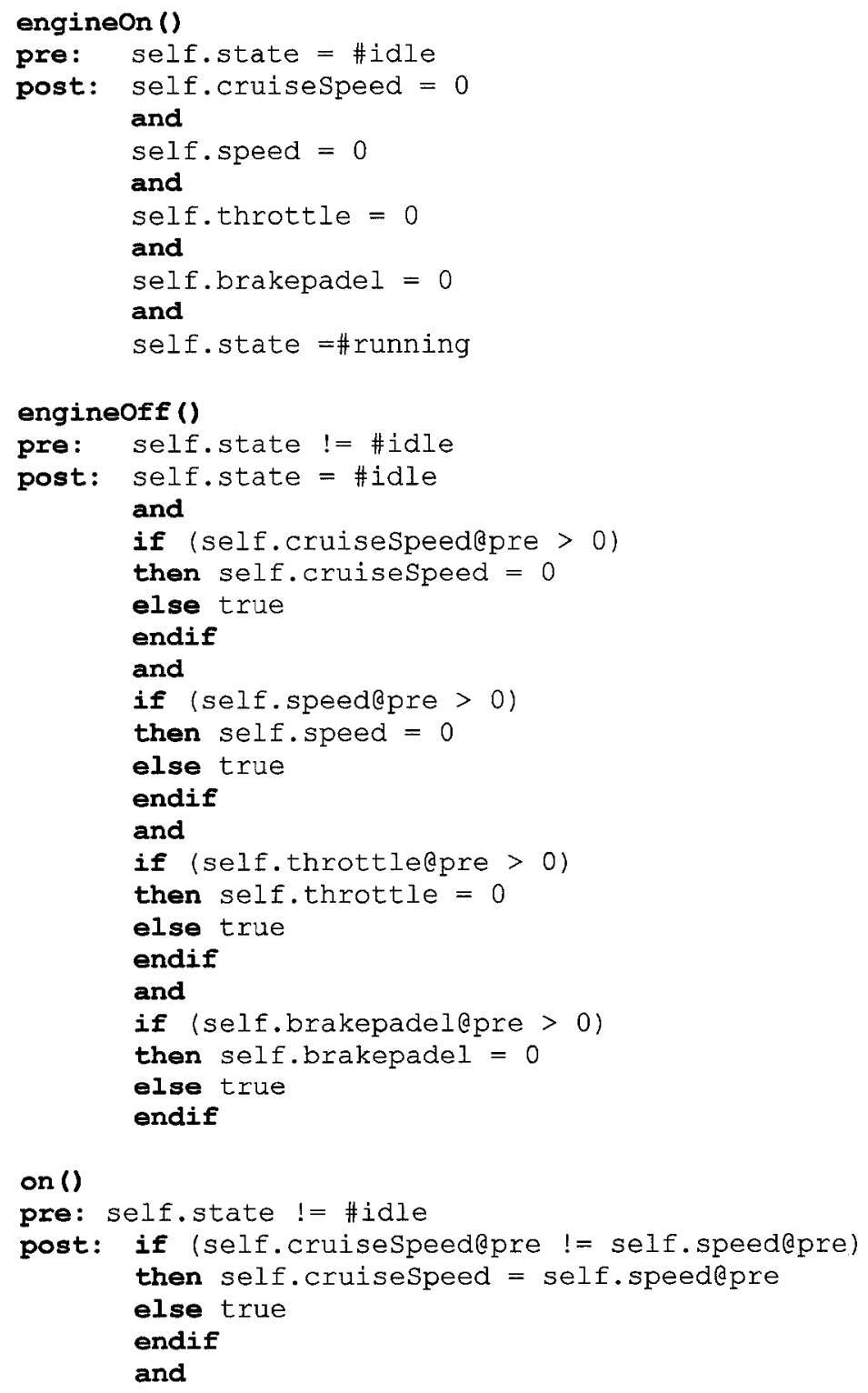


if (self.cruisespeed $>0$ )

Term5

then self.throttle = self.cruiseSpeed/Cruisecontroller.st

+ (self.cruisespeed - self.speed)/CruiseController.diff Term6

else true

Term7

endif

and

if (self.state@pre != \#cruising) Term8

self.state =\#cruising

Term 9

accelerate ()

Pre: $\quad$ self.state $=$ \#idle

Post: if (self.brakepedalepre >0)

then self.brakepedal $=0$

else true

endif

and

if (self.throttlelpre < Cruisecontroller.maxThrottle - 5.0) Term5

then self.throttle = self.throttledpre $+5.0 \quad$ Term6

else self.throttle = CruiseController.maxThrottle $\quad$ Term7

endif

and

if (self.speedepre < self.maxspeed)

Term1

Term2

Term3

Term4

then

if ( (self.throttle - self.speedepre / CruiseController.airRes)

/ CruiseController.speedRate)

< CruiseController.maxspeed-self.speedapre) Term9

then self. speed $=$ self.speedepre

+ (self.throttle - self.speedepre/CruiseController.airRes)

/ Cruisecontroller. speedRate

else self. speed $=$ CruiseController.maxspeed $\quad$ Term11

endif

else true

Term12

endif

and

if (self.stateepre = \#cruising)

Term13

then self.state = \#standby

Term14

else true

Term15

endif

Term16

\section{brake ()}

Pre: $\quad$ self.state! $=\# i d l e$

Post: if (self.throttle@pre > 0.0)

Terml

then self.throttle $=0.0$

else true

Term2

endif

and

if (self.brakepedalepre < CruiseController.maxBrake - 1) Term5

then self.brakepedal $=$ self.brakepedalepre $+1 \quad$ Term6

else self.brakepedal = CruiseController.maxBrake

Term7

endif

and

if (self.speedepre $>0$ )

Term8

then

if ( (self.speedepre / Cruisecontroller.airRes - 2* self.brakepedal)

/ \#speedRate < self. speedepre)

Terms

then self. speed = self. speedepre

- (self.speed@pre / Cruisecontroller.airRes

- 2*self.brakepedal)/CruiseController.speedRate Term10

elso self.speed $=0$

Term11

endif

else true

Term12

endif

and 
if (self.statedpre = \#cruising)

Term13

then self.state = \#standby

Term14

else true

Term15

endif

off ()

pre: self.state $=$ \#cruising

Term1

post: self.state =\#standby

Term2

resume()

pre: self.state $=$ \#standby

Term1

post: if (self.cruiseSpeedepre >0)

then self.throttle = self.cruisespeed / CruiseController.st + (self.cruiseSpeed - self.speed) / CruiseController.diff Term3

else true

Term4

endif

and

self. state =\#cruising

Term5

\section{F.2 Operation contracts for Ordered Set}

Attributes:

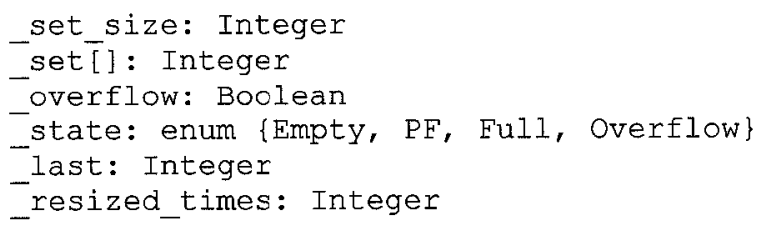

Methods:

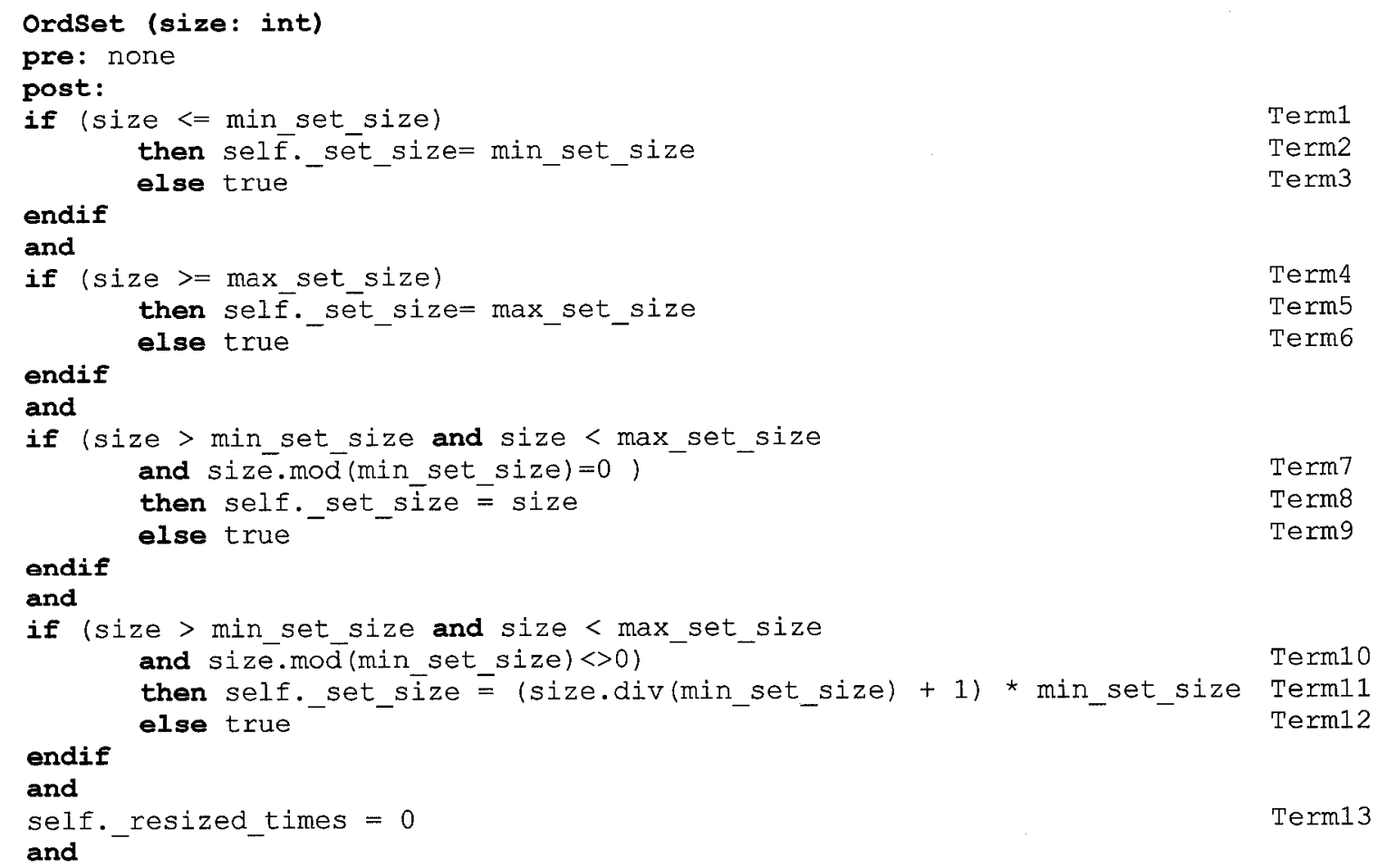




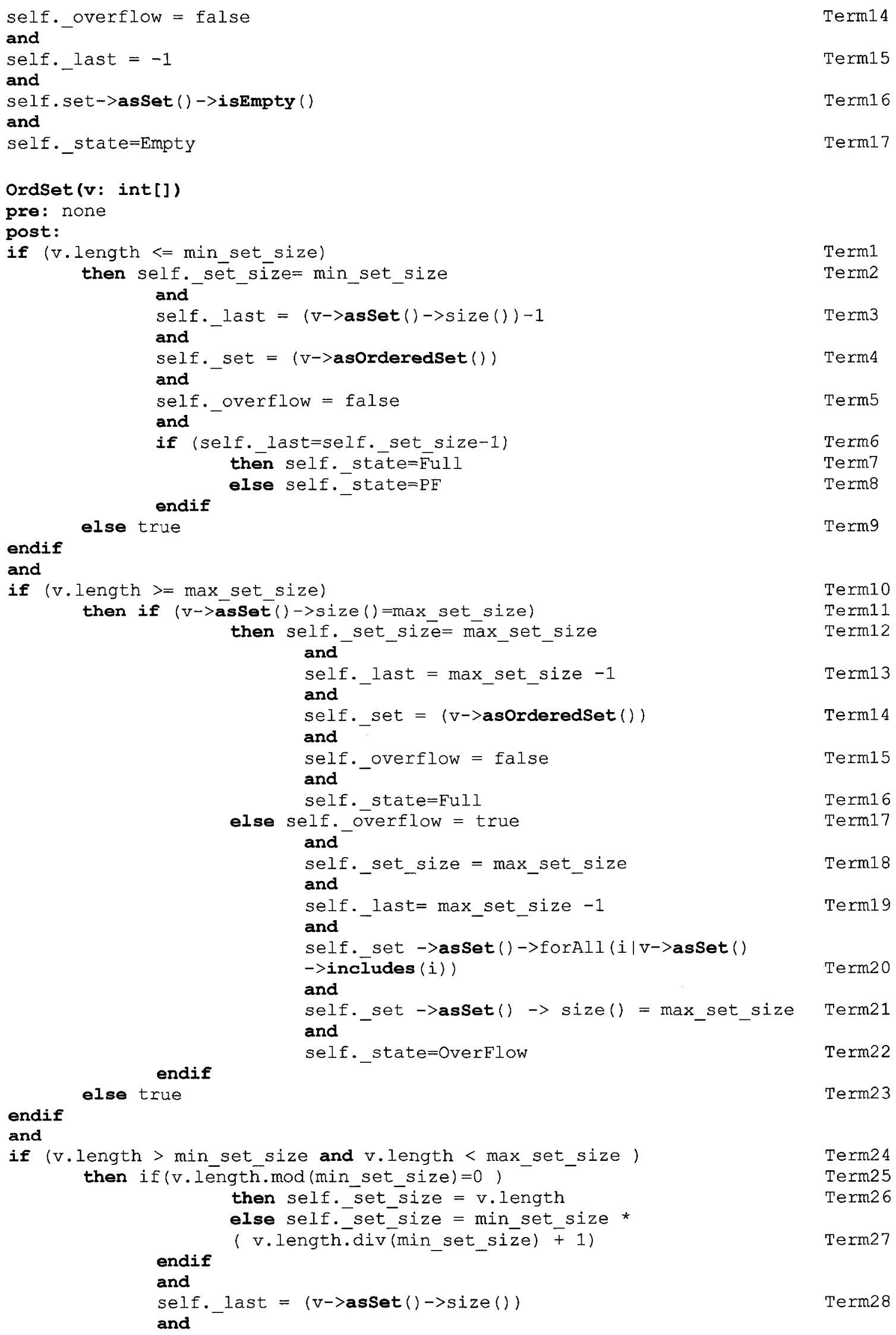




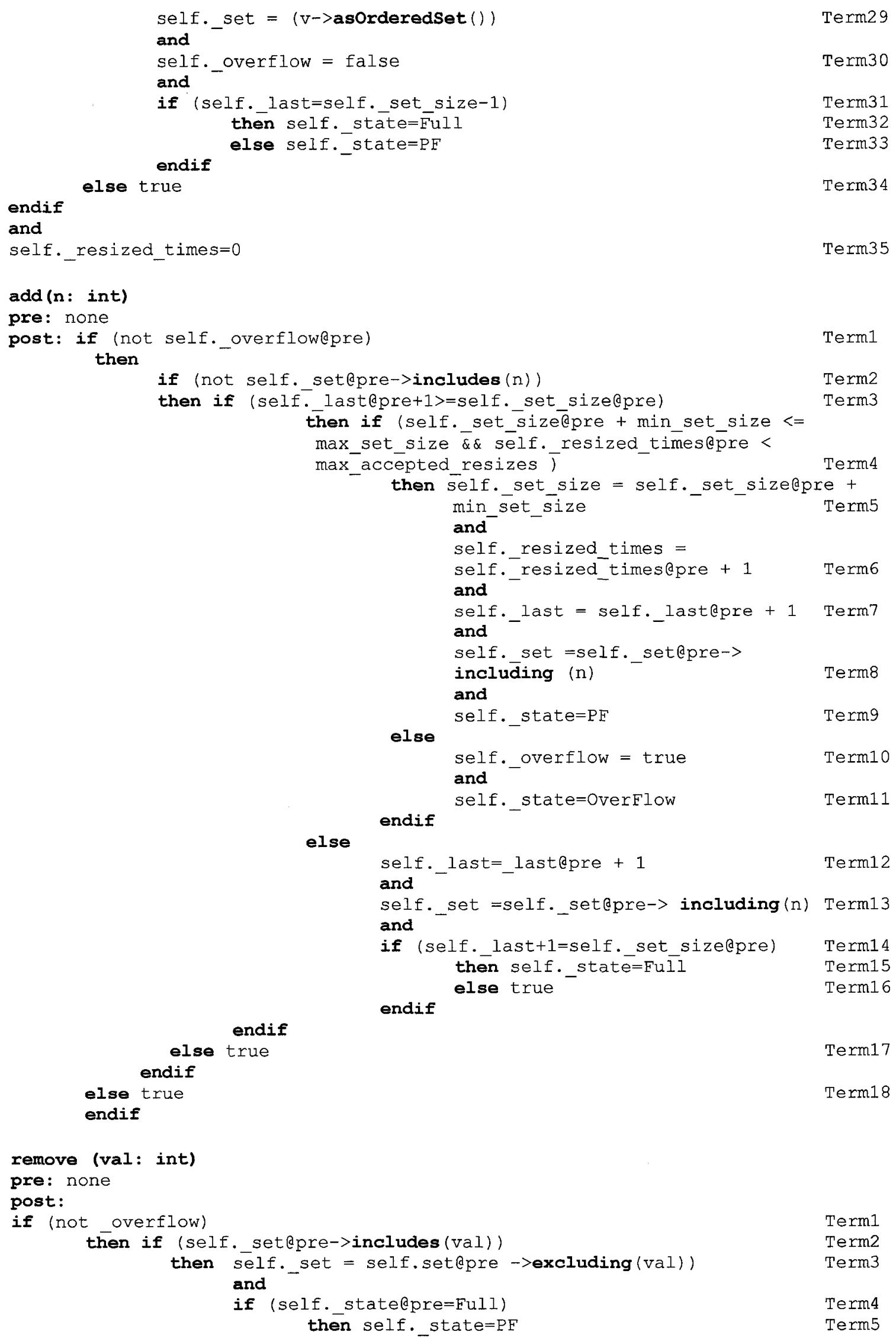




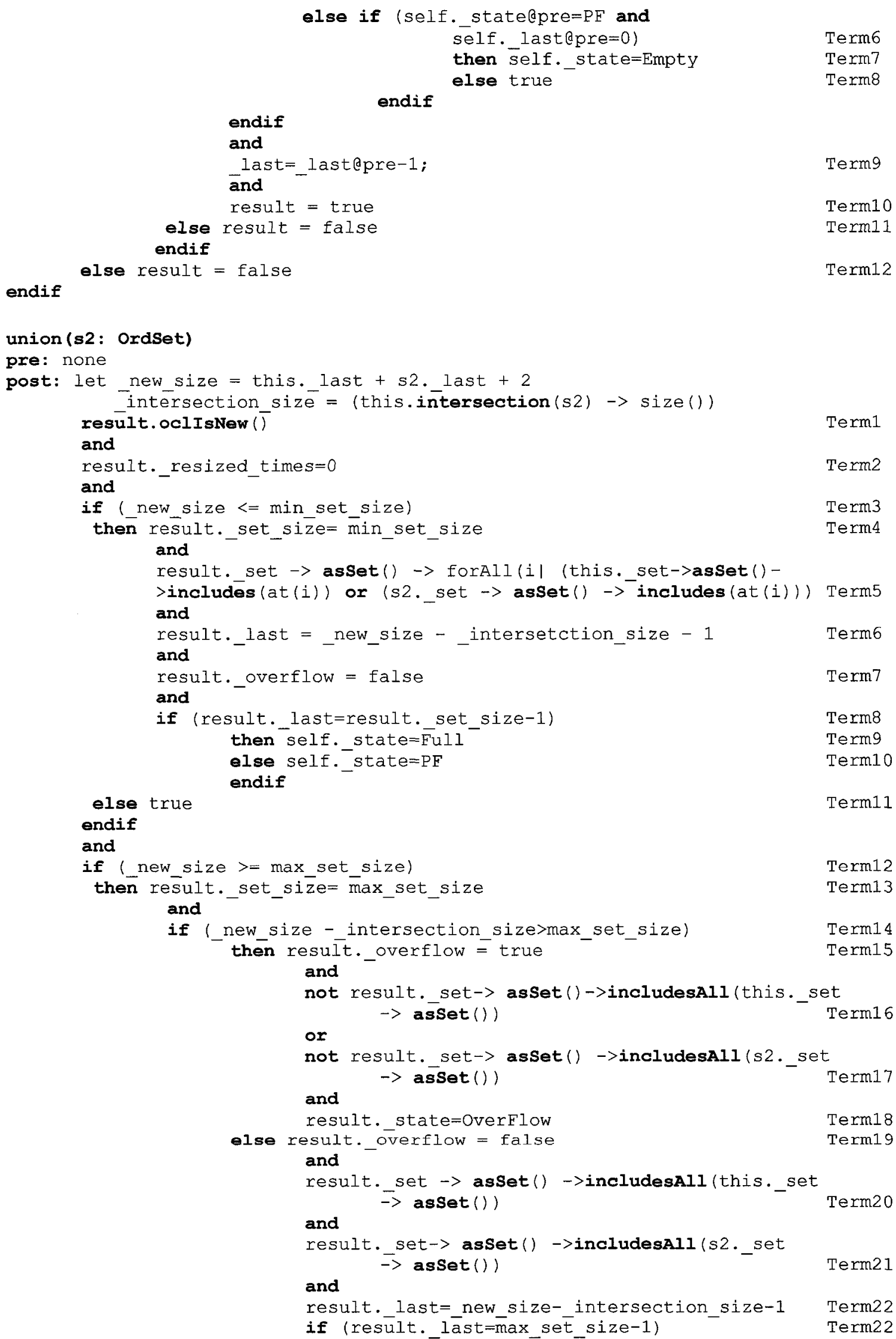




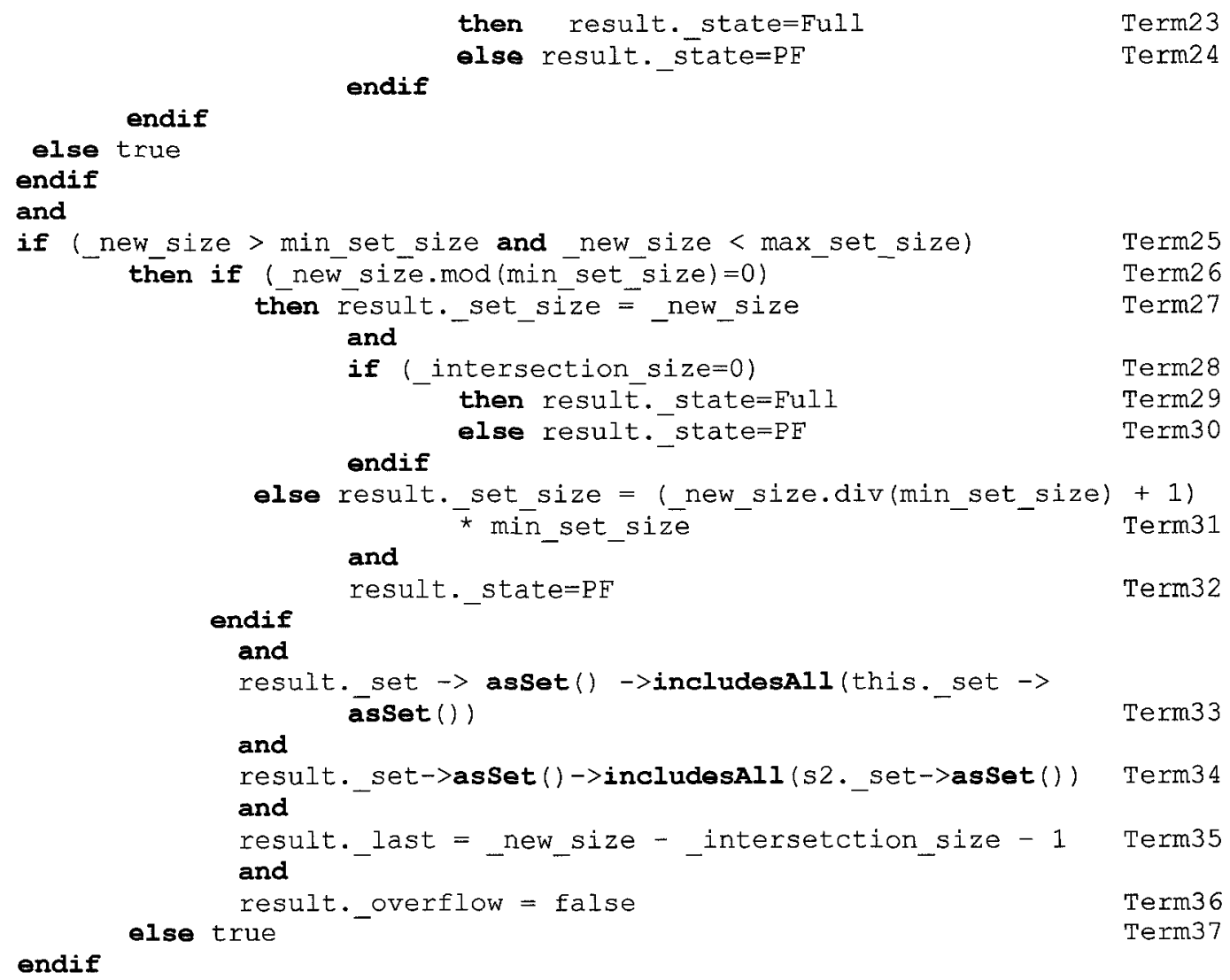

\section{F.3 Operation contracts of VCR system:}

Attributes:

state: enum foff, TapeAbsent, Playing, Forwarding, Rewinding, Recording, RecPaused, Stopped, Paused

isTapePresent: Boolean

numTimesTapePulled: Integer

isTapePulledToDrum: Boolean

tape: Tape

eventStartTime: Integer

Methods:

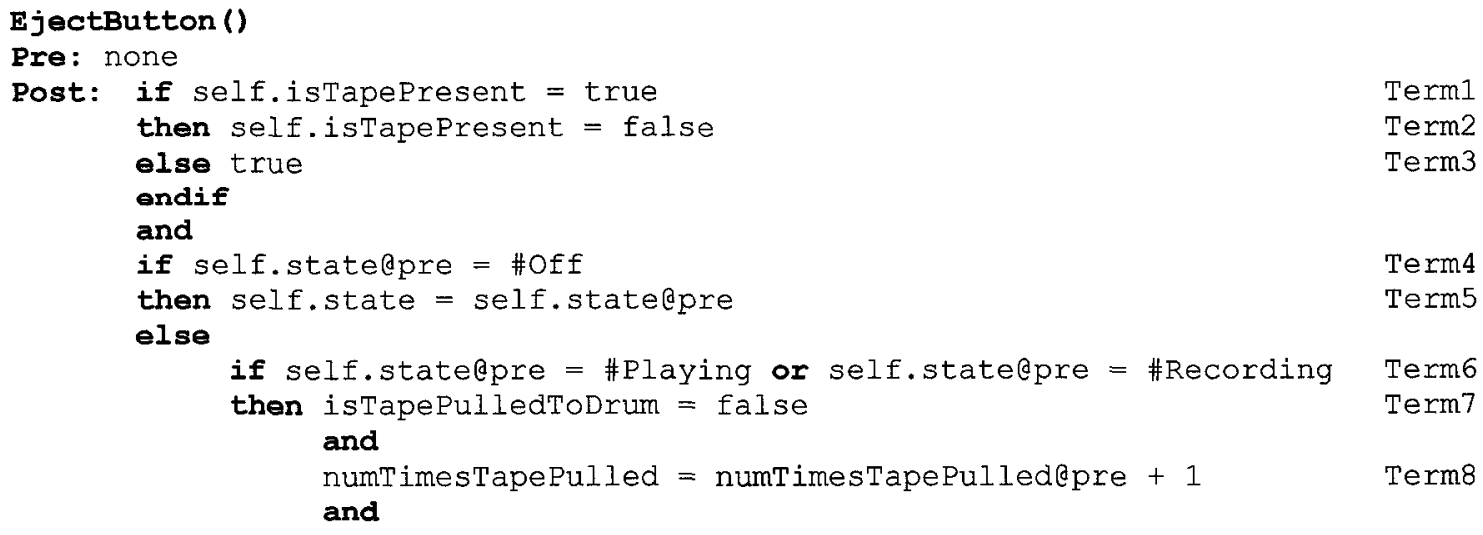




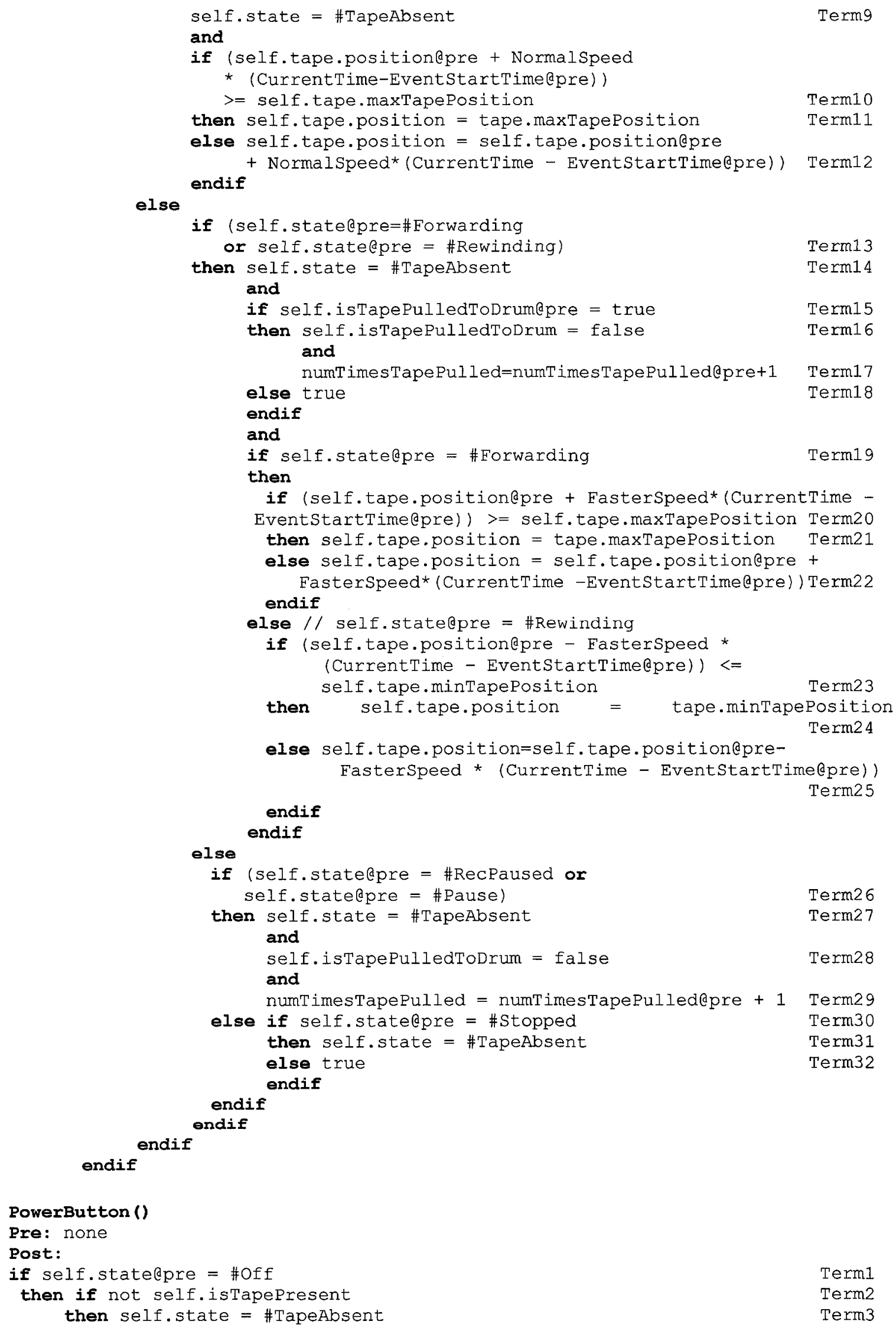




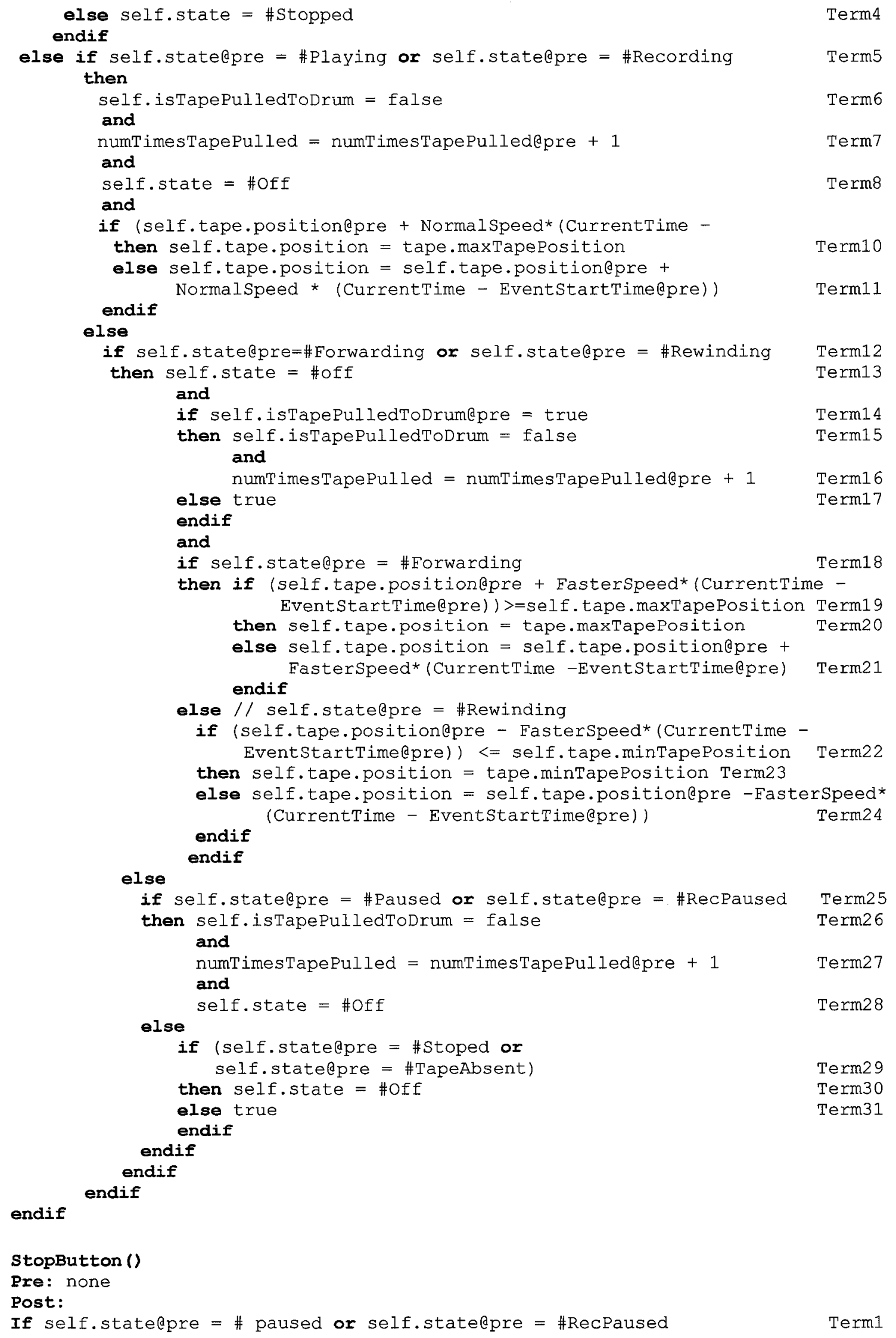




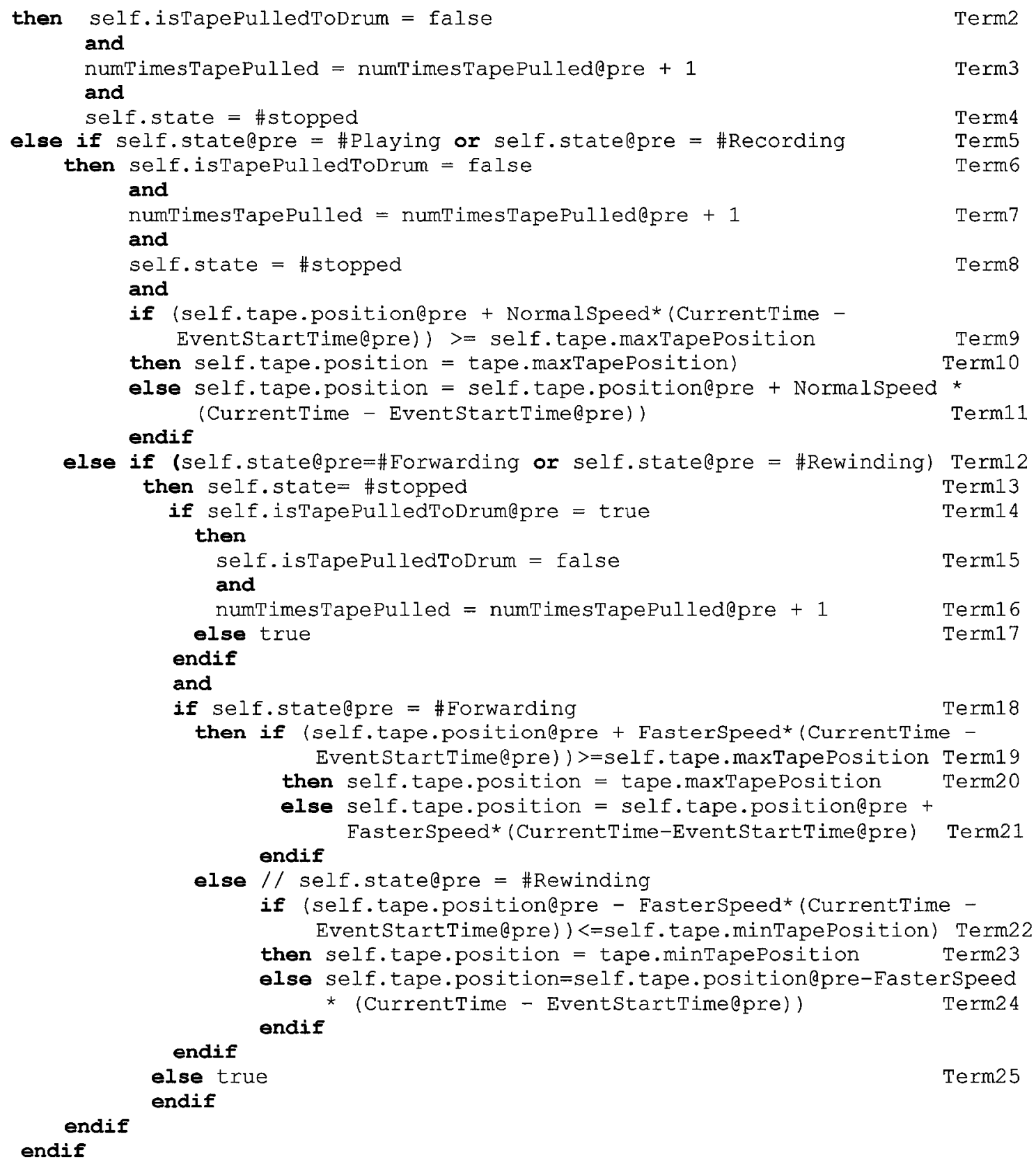




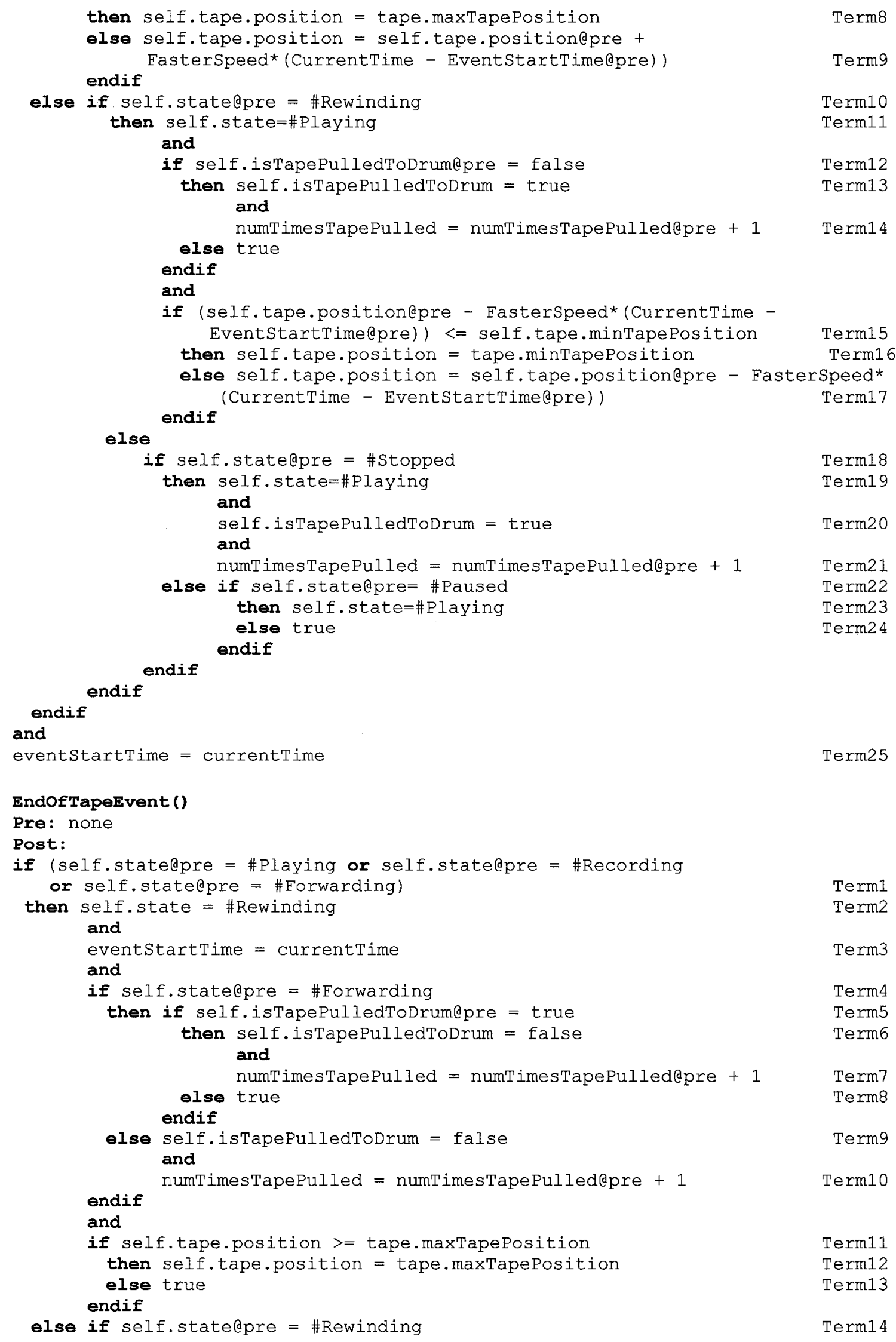




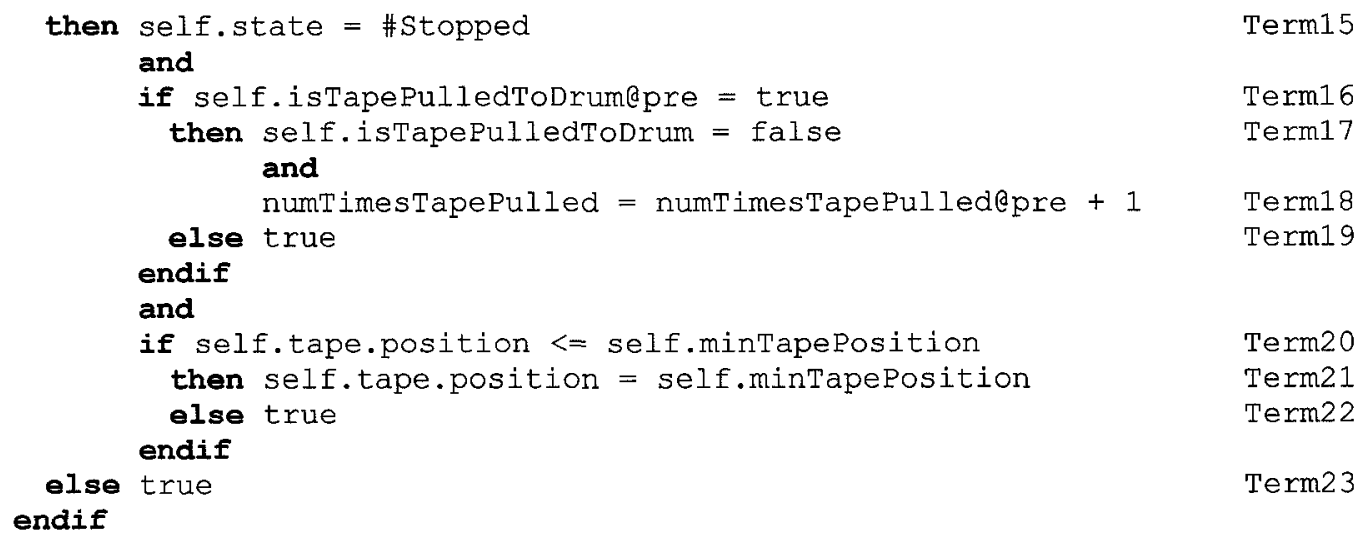




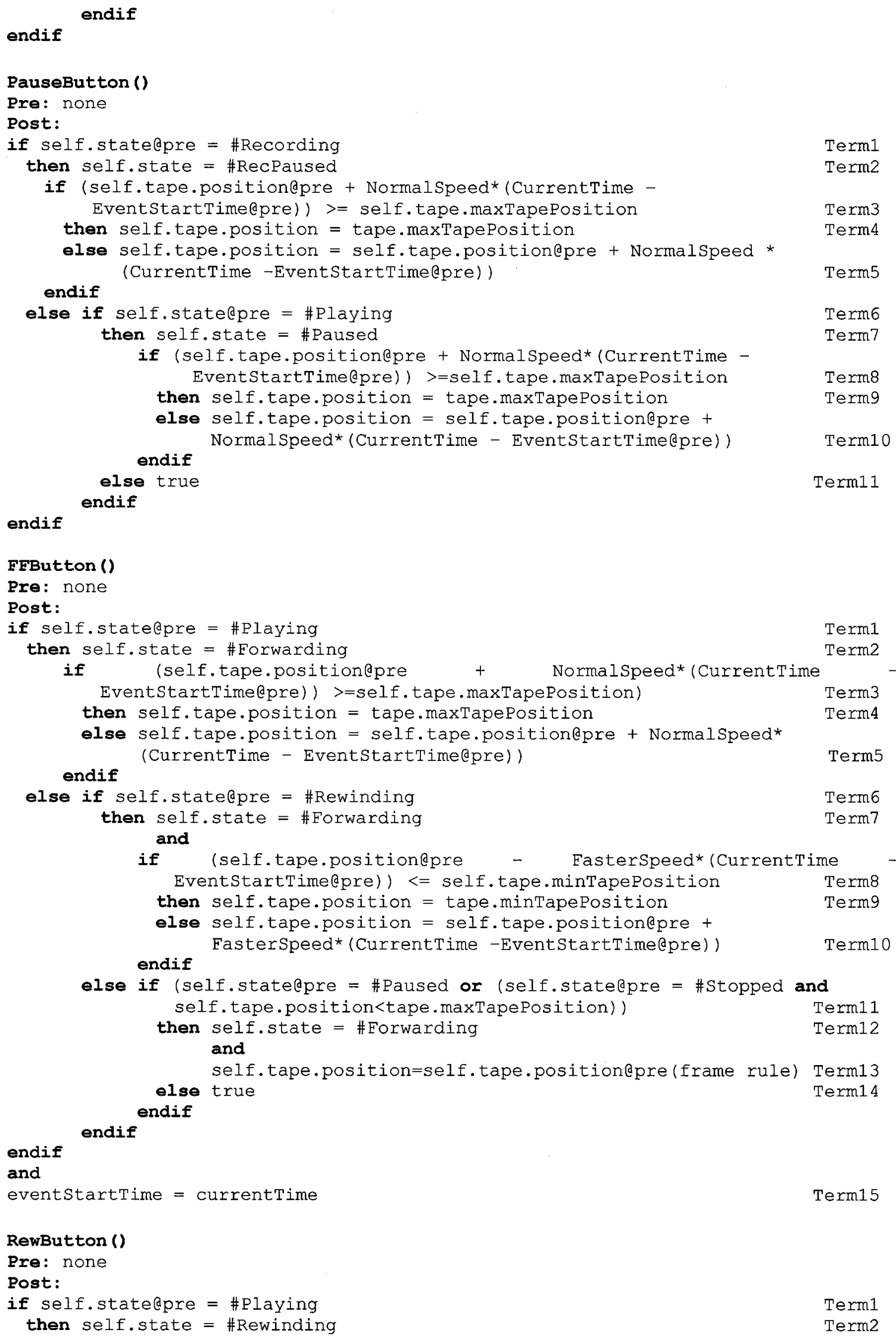




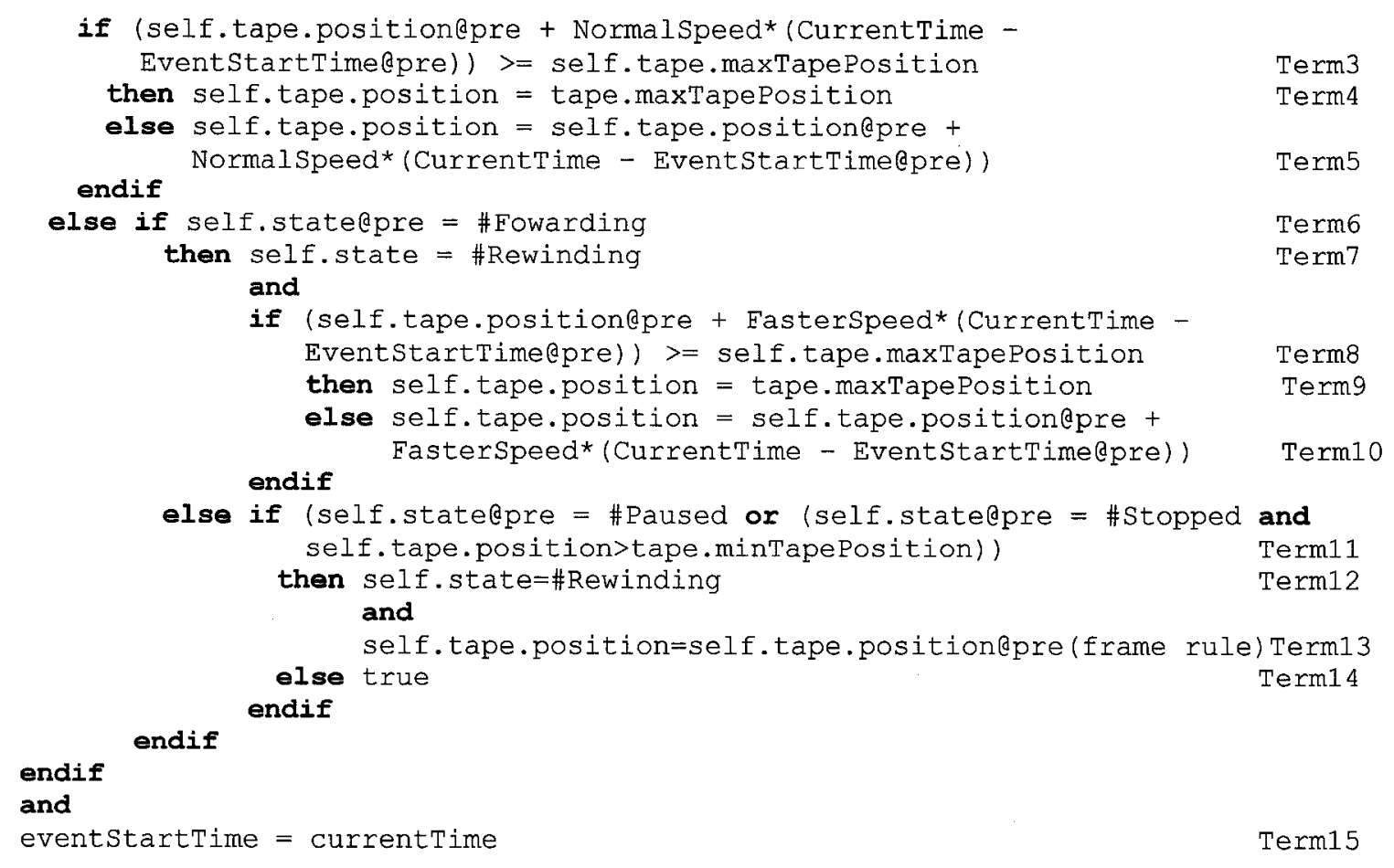

